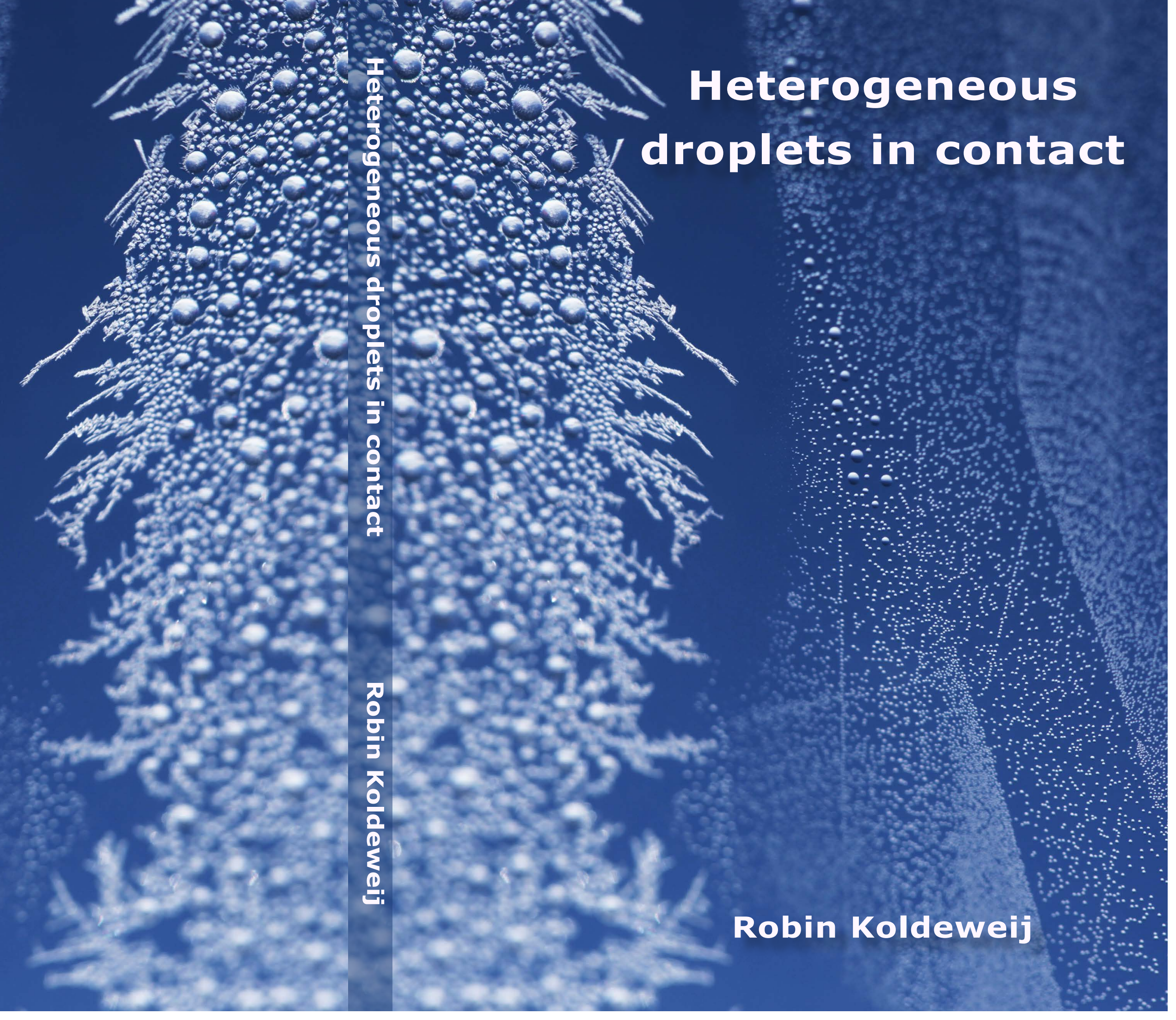





\section{HETEROGENEOUS DROPLETS IN CONTACT}

Robin Bernardus Johannes Koldeweij 


\section{Samenstelling promotiecommissie:}

Prof. dr. J.L. Herek (voorzitter)

Prof. dr. rer. nat. Detlef Lohse (promotor)

Prof. dr. ir. J.H. Snoeijer

Prof. dr. ir. S.J.G. Lemay

Prof. dr. C. Josserand

Prof. dr. ir. J.A.M. Kuipers
Universiteit Twente

Universiteit Twente

Universiteit Twente

Universiteit Twente

CNRS et Université Pierre et Marie Curie

Technische Universiteit Eindhoven


The work in this thesis was carried out at the Physics of Fluids group of the Faculty of Science and Technology of the University of Twente. It is part of the early research programme of Nano-Optomechatronics Instruments, within the Netherlands Organisation for Applied Scientific Research (TNO). Coverpicture adapted from MisterQM/photocase.com

Nederlandse titel:

HETEROGENE DRUPPELS IN CONTACT

Publisher:

RBJ Koldeweij, Physics of Fluids, University of Twente, P.O. Box 217, 7500 AE Enschede, The Netherlands pof.tnw.utwente.nl

(C) RBJ Koldeweij, Enschede, The Netherlands 2020

No part of this work may be reproduced by print photocopy or any other means without the permission in writing from the publisher ISBN: 978-90-365-5054-3 


\section{HETEROGENEOUS DROPLETS IN CONTACT}

\section{PROEFSCHRIFT}

ter verkrijging van

de graad van doctor aan de Universiteit Twente,

op gezag van de rector magnificus,

Prof. dr. T.T.M. Palstra,

volgens besluit van het College voor Promoties

in het openbaar te verdedigen

op vrijdag 25 September 2020 om 16.45 uur

door

Robin Bernardus Johannes Koldeweij

geboren op 21-03-1984

te Winterswijk 
Dit proefschrift is goedgekeurd door de promotor:

Prof. dr. rer. nat. Detlef Lohse 


\section{Contents}

1 Introduction 1

1.1 Multi-material additive manufacturing . . . . . . . . . . 1

1.2 Inspiration . . . . . . . . . . . . . . 2

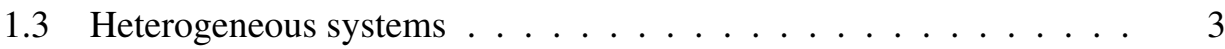

1.4 Guide through this thesis $\ldots \ldots \ldots \ldots$

2 Initial solidification of spreading droplets 11

2.1 Introduction . . . . . . . . . . . . . . . . . 12

2.2 Experimental methods . . . . . . . . . . . . . . . 13

2.3 Results and interpretation . . . . . . . . . . . . . . 13

2.4 Conclusions . . . . . . . . . . . . . . . . . . . . . . . 19

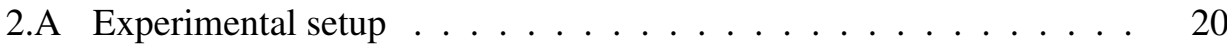

2.B Material properties . . . . . . . . . . . . . 20

2.C Visualisation . . . . . . . . . . . . . . . . 20

2.D Nucleation rate . . . . . . . . . . . . . . . . 22

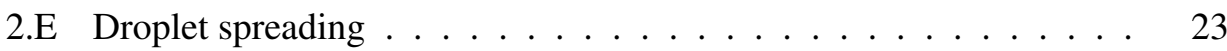

3 Impacting droplet on a cold surface $\quad 29$

3.1 Introduction . . . . . . . . . . . . . . . 30

3.2 Results and Discussion . . . . . . . . . . . . . . . 30

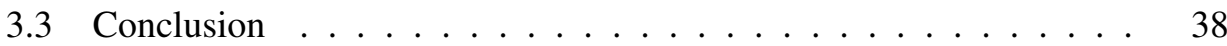

3.A Materials and Methods . . . . . . . . . . . . . . . . . 39

3.B Two-phase Stefan problem . . . . . . . . . . . . . . . . 40

3.C Kinetic model for cluster growth: Interface controlled growth . . . . 42

3.D Visualisation ..................... 45

4 Droplet impact on a supercooled surface and its subsequent solidification $\mathbf{5 3}$

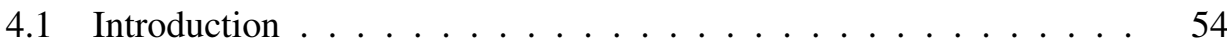

4.2 Numerical model . . . . . . . . . . . . . . . . . . . . 56

4.3 Validation \& sensitivity for impact of tin droplets . . . . . . . . . 61

4.4 Results . . . . . . . . . . . . . . . . . . . . . 63

4.5 Conclusions and Outlook . . . . . . . . . . . . . . . . 74 
4.A Parameter sensitivity . . . . . . . . . . . . 75

5 Marangoni-driven spreading $\quad 87$



5.2 Experimental set-up and materials $\ldots \ldots \ldots \ldots \ldots$

5.3 Results and discussion . . . . . . . . . . . . . . . . . . . . 94

5.4 Conclusions . . . . . . . . . . . . . . . . . . . 100

5.A Range of control parameters . . . . . . . . . . . . . . . 101

5.B Surface tension measurements . . . . . . . . . . . . . . . 101

5.C Viscosity measurements . . . . . . . . . . . . . . . . . 102

5.D Optical penetration depth . . . . . . . . . . . . . . 102

5.E Boundary layer thickness . . . . . . . . . . . . . . . . . . 102

6 A local curve fitting method to model variable surface tension 113

6.1 Introduction . . . . . . . . . . . . . . . . . . . . . . . 114

6.2 Numerical method . . . . . . . . . . . . . . . . . . . . . 114

6.3 Validation . . . . . . . . . . . . . . . . . . . . . . 119

6.4 Conclusion and outlook . . . . . . . . . . . . . . . . . 125

6.A Analytical results to the stacked liquid layers . . . . . . . . 126

6.B Analytical results to the migrating droplet in 2D. . . . . . . . 126

$\begin{array}{lll}7 & \text { Conclusions and Outlook } & 137\end{array}$

8 Summary 139 




\section{1 \\ Introduction}

\subsection{Multi-material additive manufacturing}

Additive manufacturing, or 3d-printing, has been in development for over a century. The first methodology to build materials in a layer wise fashion was developed to produce contour relief maps, as early as in 1892 [1]. Subsequently, a wide range of additive manufacturing methods have been developed, an extensive overview is given in Ref [2]. Since 2010 there has been a standard for the terminology of the range of technologies, that have been classified into 7 categories by ISO [3]. These range from focused thermal energy to locally melt materials (directed energy deposition and powder bed fusion), depositing a liquid to join powder (binder jetting), using lightactivated polymerisation (vat photopolymerisation), bonding sheets (sheet lamination), to direct material deposition, either through extrusion (material extrusion) or through inkjet printing (material jetting).

The development of additive manufacturing has mainly focused on building single material products via either of these methods. All technologies have a variety of applications, like rapid prototyping of test products or producing functional parts composed of polymers or metals. More recently, additive manufacturing technologies have been developed to build products composed of multiple materials.

Of the variety of additive manufacturing techniques, a promising technique to create multi-material products is material jetting. With material jetting, each successive layer is directly deposited from a material processed by a printhead, drop by drop. Every single droplet can be composed of an individual compound. Therefore, a single layer can be built up from several materials. This flexibility leads to a wide array 
(a)

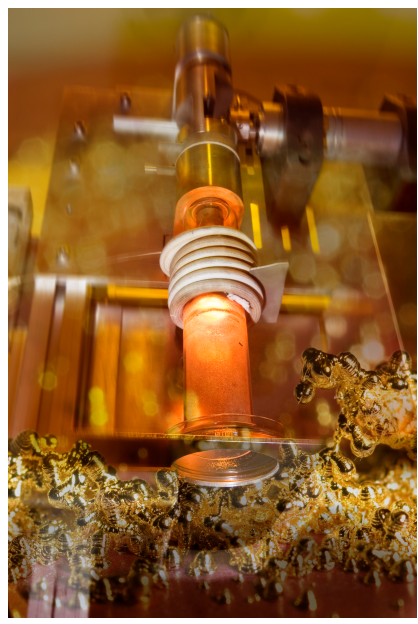

(b)

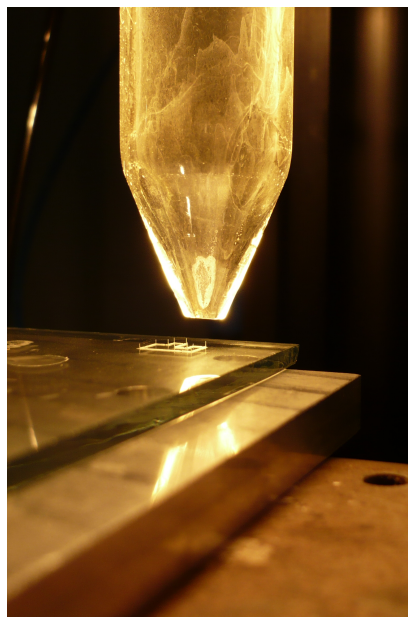

Figure 1.1: Inkjet systems for metal printing. (a) A pyrolysis printer. (b) A direct metal jetting system.

of possibilities to create complex products. Early methods could print multi-colored objects of the same type of material [4]. This has evolved towards the printing of credible dentures [5]. A further development, is the combination of materials with a broader range of properties. This leads to a vast array of new possibilities. For instance complex electronic structures can directly be printed on parts, either as a separate production step, or directly during printing of the main part [6-8].

\subsection{Inspiration}

TNO has developed technologies to deposit metal droplets to print $3 \mathrm{~d}$ dimensional electronics. Two main branches of systems have been considered.

- inkjet technology based on the printing of a solvent laden with a salt precursor, such as $\mathrm{AgNO}_{3}$. After evaporation of the solvent and pyrolysis of the precursor, solid silver is obtained (figure 1.1(a)) [9].

- inkjet technology based on the direct jetting of metals (figure 1.1(b)) up to a melting point of $1400^{\circ} \mathrm{C}[10]$.

Both technologies have been shown to be able to successfully create conductive metal tracks on a variety of (non-metallic) substrates, giving confidence that multimaterial printing with metals is feasible. Several results of these experiments are shown in figure 1.2. These experiments are mainly performed to investigate the capabilities of the printheads [9]. Next to the development of systems required to jet 


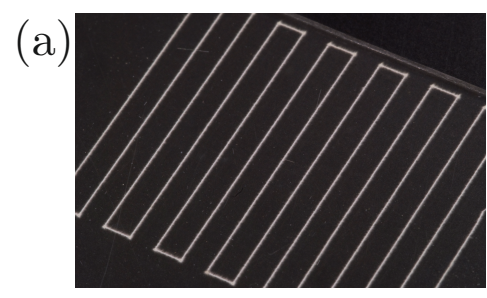

(b)

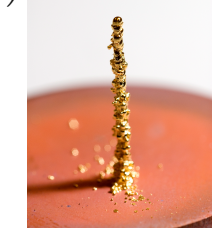

(c)

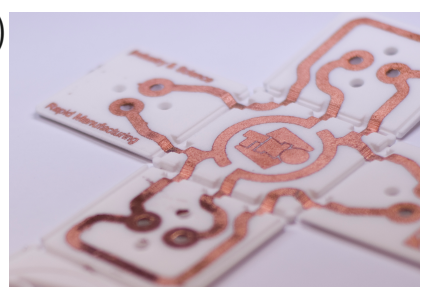

Figure 1.2: Experimental results for metal printing (a) A conductive track on glass. (b) A pillar of gold. (c) Copper tracks on 3D-printed nylon.

metal droplets, the interaction of these droplets with each other and with the substrate lead to complex phenomena, due to the large variety of thermal and liquid properties involved. To create a functional 3d-printed product, this complex interplay between droplet hydrodynamics in combination with the phase change during the deposition of droplets on a substrate needs to be understood. Furthermore the interplay between droplets of different composition needs to be controlled to manufacture an adequate product. The development of methods to further study this interaction of the droplets with each other and with the substrate is the topic of this thesis.

\subsection{Heterogeneous systems}

Two types of heterogeneous systems are of importance during 3d-printing: firstly those where a phase change occurs during the relevant time scale of the ongoing process, either the evaporation of a solvent, or the solidification of the printed material. Secondly those where the thermal or hydrodynamical properties of the materials in contact are significantly different.

\subsubsection{Solidification}

When a liquid is cooled below its freezing point, a phase transition takes place where it changes into a solid. This solidification process takes place at several length scales, ranging from $10^{-9}$ to $10^{-6} \mathrm{~m}$, see Figure 1.3 . The individual size of droplets that are inkjetted are typically in the scale of $10^{-4}$ to $10^{-3} \mathrm{~m}$, so the various length scales all need to be understood in detail to control the droplet deposition process.

- On the macroscopic scale, down to the order of $10^{-3} \mathrm{~m}$, two phases can be determined, separated by a solid-liquid interface. On this scale, the solidification process behaves as a continuum. The solid-liquid interface is a moving boundary, where latent heat is released during the phase transformation. The temperature distribution and sharp solidification front can be described by the Stefan problem 


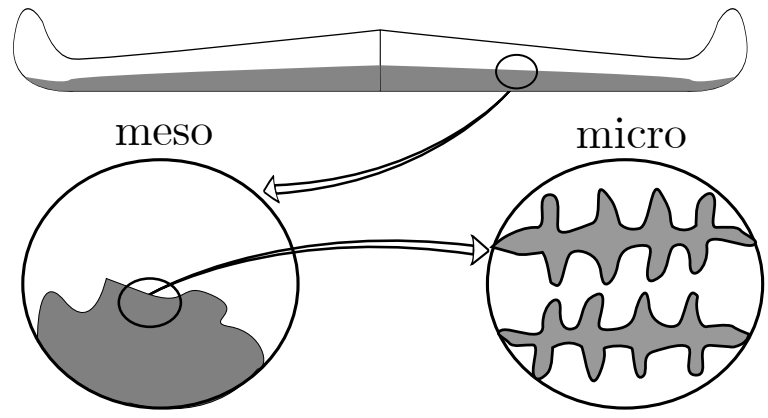

Figure 1.3: Artist impression of the length scales of solidification during droplet spreading. The sharp interface of the macroscopic view of the solidified materials a large amount of features on a mesoscopic scale. On the microscopic scale individual crystallites can be observed.

[11]. For solidification in one dimension, the moving solid front is canonically described by the Schwarz solution to this Stefan problem [12], which describes the complete temperature field as well as the time dependent position of the solid front.

- On a mesoscopic scale, in the order of $10^{-4} \mathrm{~m}$, more striking features of solidification can be observed. The solid-liquid interface is considerably more complex with rich unstable behavior [13]. There is no clear contrast between the liquid and the solid. The region containing the liquid-solid interface is often denoted as the mushy zone.

- On a nanoscopic to microscopic length scale $10^{-9}$ to $10^{-6} \mathrm{~m}$, it becomes clear that the condition of cooling is not sufficient for a system to solidify. In the liquid, a number of nuclei must first develop, that act as centers of crystallization. As soon as a stable nucleus is formed, it can grow into solid crystals by transfer of individual atoms from the liquid to the solid.

The difference between the Gibbs free energy $G=H-T S$ of the liquid and the to be formed solid acts as the driving force for crystallization. $T$ and $S$ are the temperature and entropy of the system; the enthalpy $H=U+P V$, with the inner energy $U$, pressure $P$ and volume $V$. Crystallization is a first order phase transition. Neglecting the surface area of the to be formed nucleus, the change of free energy for isobaric-isothermal crystallization from phase 1 to phase 2 can be written as:

$$
\Delta G=U_{2}+P V_{2}-T S_{2}-U_{1}-P V_{1}+T S_{1} .
$$

For very small volume changes, $U \approx H$, so $\Delta G=\Delta H-T \Delta S$. the change of entropy 
$\Delta S=q / T$, where $q$ is the amount of energy required for the phase change, is usually very high for crystallization processes.

\subsubsection{Marangoni effects}

The free surface of a liquid, where in the typical scale of 3d-printed droplets, the interfacial tension with the air is of fundamental importance to the process. A variation in liquid properties leads to a further heterogeneity. Local variations of interfacial tension leads to mass transport of liquid on the free surface, the so called Marangoni effect, named after the studies of Carlo Marangoni [14], and first observed in alcoholic beverages by James Thomson [15]. For instance, spontaneous flow can develop near an interface across which species are transferred. If the interfacial tension decreases with increasing concentration of a certain solute, the resulting interfacial tension gradient leads to a flow that transfers liquid from the solution rich phase to a solution lean phase.

The interfacial tension can vary with both position and time, due to any quantity that affects surface tension, such as temperature $[16,17]$, the presence of surfactants $[18,19]$, or a difference of concentration in a multi-component system [20, 21].

Temperature dependence can have a further effect on Marangoni flow. When large temperature gradients occur or during the phase change of a material, the surface tension can change dramatically, leading to severe Marangoni induced flow. For instance in the melt pool during the laser melting of a powder [22, 23]. Similarly, for droplets, it has been shown that for melting [24], solidification [25], or evaporation [26], a variation in surface tension can have a significant effect on the hydrodynamics.

\subsection{Guide through this thesis}

The complexity of multi-material $3 \mathrm{~d}$ printing served as an inspiration to study droplet solidification on substrates and liquids in contact with other liquids. In this thesis, numerical methods and experimental techniques are developed to study these processes.

Initially, the solidification behavior of spreading and impacting droplets is studied experimentally. To this effect, we present a novel experimental setup in 2 to demonstrate the influence of nucleation and crystal growth near the droplet-solid interface. In chapter 2 we use this setup to study droplets with no impact velocity, whilst in chapter 3 we look at impacting droplets. Subsequently, to study the effect of solidification on the full droplet scale, we present a numerical method in chapter 4 . Here the impact behavior of single tin droplets is described.

The effects of heterogeneity in material properties are studied experimentally in chapter 5. Here, laser-induced fluorescence is used to study the spreading of 
binary droplets for a large range of viscosities and surface tensions. In chapter 6 a new numerical method is proposed and validated, which allows the computation of liquid-liquid systems with varying surface tension.

Finally, chapter 7 gives an overview of the conclusions of this thesis. 


\section{References}

[1] J. E. Blanther, "Manufacture of contour relief-maps", (1892), wO2009/011583 A1.

[2] P. Torres, J. Esau, et al., "Rapid prototyping and manufacturing: A review of current technologies", (2009).

[3] "Additive manufacturing - General principles - Terminology", Standard, International Organization for Standardization (2015).

[4] R. Helinski, "Method and means for constructing three-dimensional articles by particle deposition", (1992), uS patent nr. 5,136,515.

[5] P. Adusumilli and S. J. Lech, "System and method for manufacturing full and partial dentures", (2007), uS2009/0287332 A1.

[6] S. J. Leigh, R. J. Bradley, C. P. Purssell, D. R. Billson, and D. A. Hutchins, "A simple, low-cost conductive composite material for $3 \mathrm{~d}$ printing of electronic sensors", PloS one 7 (2012).

[7] D. Espalin, D. W. Muse, E. MacDonald, and R. B. Wicker, "3d printing multifunctionality: structures with electronics", The International Journal of Advanced Manufacturing Technology 72, 963-978 (2014).

[8] E. Macdonald, R. Salas, D. Espalin, M. Perez, E. Aguilera, D. Muse, and R. B. Wicker, "3d printing for the rapid prototyping of structural electronics", IEEE access 2, 234-242 (2014).

[9] R. J. Houben, Equipment for printing of high viscosity liquids and molten metals (2012).

[10] R. J. Houben and G. Oosterhuis, "Method and apparatus for applying a material on a substrate", (2009), uS patent nr. 473,901.

[11] S. H. Davis, Theory of solidification (Cambridge University Press) (2001).

[12] H. S. Carslaw and J. C. Jaeger, Conduction of Heat in Solids (1959).

[13] D. Anderson and M. G. Worster, "Weakly nonlinear analysis of convection in mushy layers during the solidification of binary alloys", Journal of Fluid Mechanics 302, 307-331 (1995).

[14] C. Marangoni, Sull'espansione delle goccie d'un liquido galleggianti sulla superfice di altro liquido (1865). 
[15] J. Thomson, "Xlii. on certain curious motions observable at the surfaces of wine and other alcoholic liquors", The London, Edinburgh, and Dublin Philosophical Magazine and Journal of Science 10, 330-333 (1855).

[16] A. Cazabat, F. Heslot, S. Troian, and P. Carles, "Fingering instability of thin spreading films driven by temperature gradients", Nature 346, 824-826 (1990).

[17] A. Karbalaei, R. Kumar, and H. J. Cho, "Thermocapillarity in microfluidics-a review”, Micromachines 7, 13 (2016).

[18] D. P. Gaver and J. B. Grotberg, "Droplet spreading on a thin viscous film", Journal of Fluid Mechanics 235, 399-414 (1992).

[19] S. Le Roux, M. Roché, I. Cantat, and A. Saint-Jalmes, "Soluble surfactant spreading: How the amphiphilicity sets the marangoni hydrodynamics", Physical Review E 93, 013107 (2016).

[20] F. Wang, A. Choudhury, M. Selzer, R. Mukherjee, and B. Nestler, "Effect of solutal marangoni convection on motion, coarsening, and coalescence of droplets in a monotectic system", Physical Review E 86, 066318 (2012).

[21] H. Kim, K. Muller, O. Shardt, S. Afkhami, and H. A. Stone, "Solutal marangoni flows of miscible liquids drive transport without surface contamination", Nature Physics 13, 1105-1110 (2017).

[22] C. Y. Yap, C. K. Chua, Z. L. Dong, Z. H. Liu, D. Q. Zhang, L. E. Loh, and S. L. Sing, "Review of selective laser melting: Materials and applications", Applied physics reviews 2, 041101 (2015).

[23] Y. Yang, M. Knol, F. Van Keulen, and C. Ayas, "A semi-analytical thermal modelling approach for selective laser melting", Additive Manufacturing 21, 284-297 (2018).

[24] J. Khodadadi and Y. Zhang, "Effects of thermocapillary convection on melting within droplets", Numerical Heat Transfer: Part A: Applications 37, 133-153 (2000).

[25] M. Dietzel, S. Haferl, Y. Ventikos, and D. Poulikakos, "Marangoni and variable viscosity phenomena in picoliter size solder droplet deposition", J. Heat Transfer 125, 365-376 (2003).

[26] C. Diddens, H. Tan, P. Lv, M. Versluis, J. Kuerten, X. Zhang, and D. Lohse, "Evaporating pure, binary and ternary droplets: thermal effects and axial symmetry breaking”, Journal of fluid mechanics 823, 470-497 (2017). 




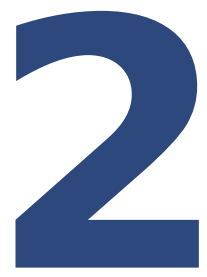

\section{Initial solidification dynamics of spreading droplets *}

When a droplet is brought in contact with an undercooled surface, it wets the substrate and solidifies at the same time. The interplay between the phase transition effects and the contact-line motion, leading to its arrest, remains poorly understood. Here we reveal the early solidification patterns and dynamics of spreading hexadecane droplets. Total internal reflection (TIR) imaging is employed to temporally and spatially resolve the early solidification behaviour. With this, we determine the conditions leading to the contact-line arrest. We quantify the overall nucleation behaviour, i.e. the nucleation rate and the crystal growth speed, and show its sensitivity to the applied undercooling of the substrate. By combining the Johnson-Mehl-Avrami-Kolmogorov nucleation theory and scaling relations for the spreading, we can calculate the temporal evolution of the solid area fraction, which is in good agreement with our observations. We also show that for strong enough undercooling it is the rapid growth of the crystals which determines the eventual arrest of the spreading contact line.

*Submitted as: "Initial solidification dynamics of spreading droplets", R.B.J. Koldeweij, P. Kant, K. Harth, R. de Ruiter, H. Gelderblom, J.H. Snoeijer, D. Lohse and M.A.J. van Limbeek 


\subsection{Introduction}

The spreading of a droplet on an undercooled surface is a very complex phenomenon as it instigates several competing physical processes simultaneously: interfacial deformation, contact-line motion and the associated fluid movement, heat exchange between the droplet and the substrate, and nucleation and growth of a solidified phase within the droplet. Understanding this process is crucial for a broad range of applications that range from ice accretion on roads [1], aircraft [2] and powerlines [3], to processes such as soldering $[4,5]$, thermal spray coating [6] and additive manufacturing $[7,8]$. So far, several investigations have addressed and characterized the intriguing macroscopic behaviour of sessile and impacting droplets on undercooled surfaces. For instance, formation of conical tips during the bulk freezing of a sessile droplet [9] and freezing kinetics along with the final splat morphology of impacting droplets have been investigated in detail [10-16]. Furthermore, nucleation has been studied using top view imaging, by applying a thermal gradient to the atmosphere $[17,18]$ or to the substrate [19]. However, nucleation and growth of crystals at the droplet-substrate interface and its subsequent influence on the droplet spreading has received only little attention [20-22]. We focus on the interplay between the various phase-transition effects and how they eventually lead to the contact-line arrest.

The arrest of a contact line on an undercooled substrate determines the size and overall shape of the final footprint between the frozen droplet and the substrate. However, due to a lack of direct visualization of the early solidification during droplet spreading, the exact mechanism responsible for contact-line arrest remains debated. So far, various experimental investigations have led to the development of the following explanations: (1) the droplet stops spreading as soon as the contact angle of the spreading liquid reaches the angle of a growing solid front [20], (2) the contact line continues to move until a critical volume solidifies in its vicinity [21], (3) the advancing motion of the droplet lasts until the local temperature falls below a threshold at which the crystal growth speed in the vicinity of the contact line becomes equal to the contact-line velocity [22].

In this chapter we reveal the sequence of events leading to the contact-line arrest using Total Internal Reflection (TIR) imaging. TIR imaging enables us to characterize the influence of substrate undercooling on the crystal nucleation kinetics as well as the tangential crystal growth along the temporally evolving wetted area. Based on these observations we propose a modelling framework that captures the freezing of an evolving droplet footprint, by combining classical nucleation theory and droplet spreading dynamics. 


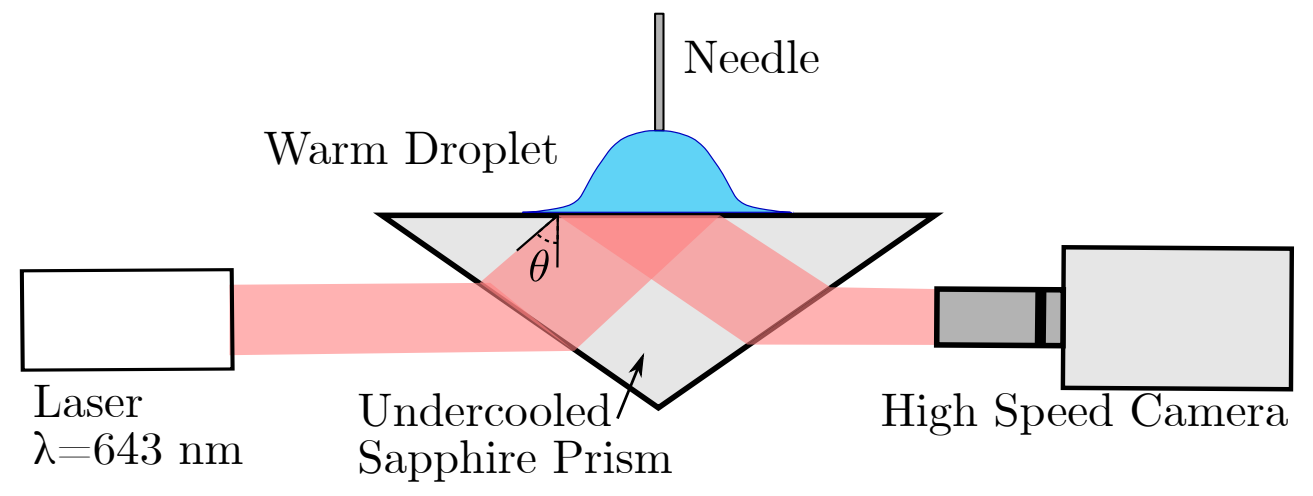

Figure 2.1: Schematic of the experimental setup for total internal reflection imaging.

\subsection{Experimental methods}

A schematic diagram of the experimental setup is depicted in figure 2.1. In a typical experiment, we inflate a droplet of hexadecane to a fixed volume, at the tip of a needle. Hexadecane has a melting point of $T_{f}=18^{\circ} \mathrm{C}$. The droplet, of radius $R_{0}=0.85 \pm 0.05$ $\mathrm{mm}$, is then gently lowered (with negligible approach velocity $U$ ) to the horizontal surface of the undercooled sapphire prism, with temperature $T_{s}<T_{f}$. Upon contact, the droplet spreading and freezing is recorded in bottom view via TIR using a high-speed camera connected to a long-distance microscope at 30000 frames per second. Note that, in contrast to the previously described TIR setups that can measure nanometric thin air-films beneath impacting droplets [23-26], our setup allows for direct visualization of the solidified phase. This is achieved by choosing the angle $\theta$ of incidence of the laser $(\lambda=634 \mathrm{~nm})$ such that total internal reflection occurs not only at the sapphire-air interface but also at the sapphire-hexadecane interface. The solidified material can be visualized owing to the localized scattering of the evanescent wave by the solid particles. Furthermore, the position of the contact line is clearly visible in the images, due to the sudden jump in refractive index between air and hexadecane. A similar setup was recently used in Ref. [16]. Details about the visualization, the experimental setup and the material properties can be found in Section 2.B.

\subsection{Results and interpretation}

Different types of solidification behaviour are observed, for different undercooling $\Delta T=T_{f}-T_{c}$. Here, $T_{c}$ is the droplet-substrate contact temperature, approximated as $T_{s}+\left(T_{d}-T_{s}\right) /\left(1+e_{s} / e_{d}\right)$ [27], with $e=\sqrt{k \rho c_{p}}$ the thermal effusivity, and the subscripts $s$ and $d$ denoting the substrate and the droplet, respectively. Sequences of snapshots in figure 2.2 show the freezing behaviours of droplets spreading on 
(a) $\Delta \mathrm{T}=1.2 \mathrm{~K}$

(b) $\Delta \mathrm{T}=2.0 \mathrm{~K}$

$3.6 \mathrm{~ms}$

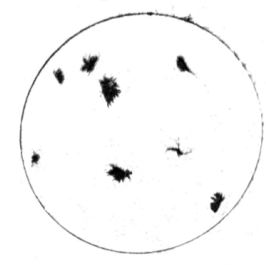

$35 \mathrm{~ms}$

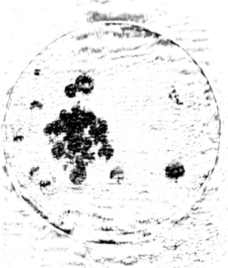

$6 \mathrm{~ms}$

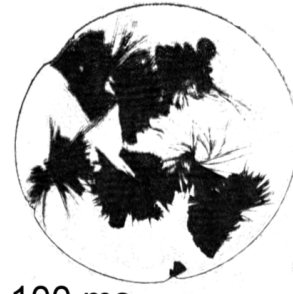

$100 \mathrm{~ms}$

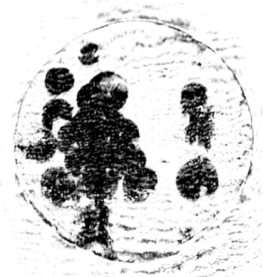

$19.7 \mathrm{~ms}$

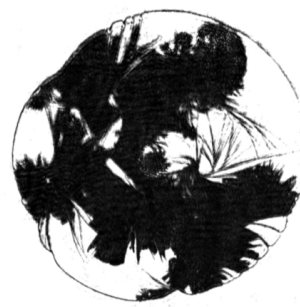

$150 \mathrm{~ms}$



$34.8 \mathrm{~ms}$

Figure 2.2: Characteristic sequences of hexadecane drops spreading on a sapphire prism of varying temperature, the red bar indicates a length of $1 \mathrm{~mm}$ : (a) $\Delta T=1.2 \mathrm{~K}$ : Random nucleation with subsequent dendritic growth. (b) $\Delta T=2 \mathrm{~K}$ : Continuous nucleation with subsequent radial crystal growth. Note the different time scales in (a) and (b).

substrates at different $\Delta T$. For low undercooling (figure 2.2(a)), nucleation initially occurs only at a few locations that are randomly distributed over the droplet footprint. Subsequently, these crystals nuclei grow into needle-shaped structures: columnar dendrites. The nucleation rate and the morphology of the growing crystals change significantly for a slight increase in undercooling. At higher $\Delta T$ (figure 2.2(b)), a considerable increase in the amount of crystals is observed. Interestingly, in this case, the enhanced nucleation rate is followed by axisymmetric growth of crystal nuclei, seen as seemingly circular footprints (figure 2.2(b)). However, a close inspection reveals that these are still constituted of dendritic patterns. Note that the phase-transition effects only initiate after a lag time $\tau_{g}$ [28]. In our experiments, $\tau_{g}$ varies from a few microseconds to a few seconds, respectively, at the largest and smallest of the $\Delta T$ employed in our experiments. The increase in the nucleation rate at higher $\Delta T$ is directly related to the corresponding decrease in the activation energy for liquid-solid transformation. For the creation of a solid nucleus, this can be considered as the sum of the surface energy between the newly created particle and the bulk, and the released latent energy in the transformed volume of this small nucleus. For a nucleus growing on a surface (heterogeneous nucleation), this critical energy can be approximated as $E_{a}=(16 \pi / 3) \gamma_{l s}^{3} f\left(\theta_{l s}\right) /(\Delta g)^{2}$ [29], with a geometrical correction factor $f\left(\theta_{l s}\right)$, that depends on the contact angle $\theta_{l s}$ of a crystalline deposit with the foreign solid surface [30]. Here $\gamma_{l s}$ is the interfacial tension between the liquid and solid hexadecane and 


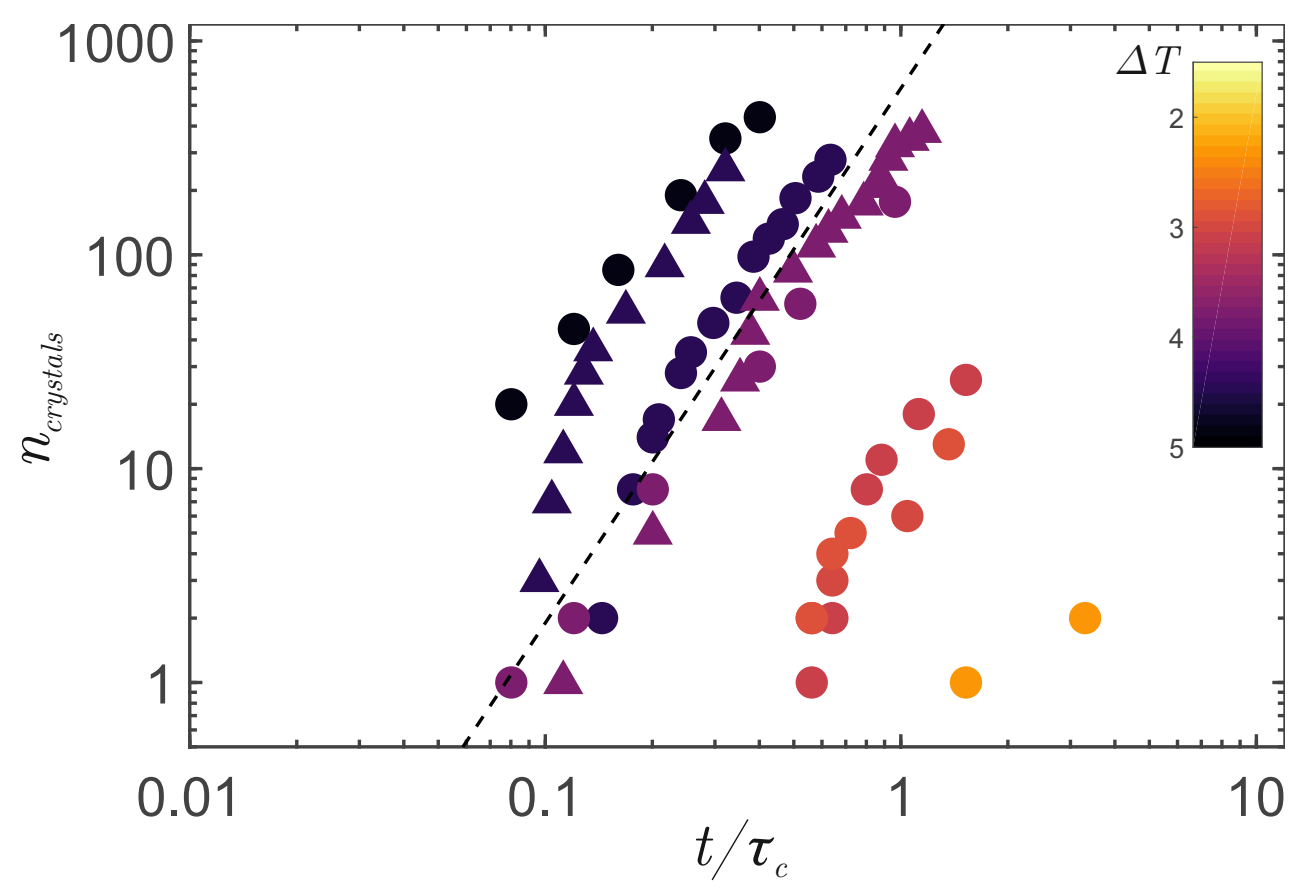

Figure 2.3: Number of distinct crystals as function of dimensionless time. Separate experiments are denoted with different symbols. The dashed line (- - ) indicates the scaling $\propto\left(t / \tau_{c}\right)^{\frac{5}{2}}$ (equation (2.1)). The capillary timescale $\tau_{c}=\left(\rho R_{0}^{3} / \sigma\right)^{1 / 2}$. The colorbar shows the temperature difference $\Delta T=T_{f}-T_{c}$.

$\Delta g=\Delta S_{f u s} \Delta T$ is the free energy difference between the liquid and solid phase, with the entropy of fusion $\Delta S_{f u s}=6.28 \cdot 10^{5} \mathrm{~J} \mathrm{~m}^{-3} \mathrm{~K}^{-1}$ [31]. Note that $E_{a}$ varies as $(\Delta T)^{-2}$. Accordingly, in our experiments for $\Delta T<1 K$, we do not observe any nucleation at the experimental timescale $(\sim 5 \mathrm{sec})$. Conversely, the droplet footprint instantly solidifies upon touching the substrate for $\Delta T>2.9 \mathrm{~K}$, when the $E_{a}$ decreases with $90 \%$.

To quantify the nucleation kinetics at the early-times of droplet spreading, we measure the total number of growing crystals $n_{\text {crystals }}$ as a function of time. The result is shown in figure 2.3 , where time is rescaled by the capillary time $\tau_{c}=\left(\rho R_{0}^{3} / \sigma\right)^{1 / 2}$, with the surface tension $\sigma$ between the liquid and air. For significantly large undercooling $\Delta T$, it follows a power-law behaviour, that we will argue to be $n_{\text {crystals }} \propto\left(t / \tau_{c}\right)^{\frac{5}{2}}$.

To rationalise this behaviour, we employ classical nucleation theory. The nucleation rate per unit volume is estimated as $J_{0}=A \exp \left(\frac{-E_{a}\left(f\left(\theta_{l s}\right)\right)}{k_{B} T_{f}}\right)$, where $k_{B}$ is the Boltzmann constant, and $A$ the attempt frequency per unit volume. The amount of crystals $n_{\text {crystals }}\left(t / \tau_{c}\right)$ is then obtained by multiplying $J_{0}$ by the available volume 
for nucleation, which is estimated by the wetted area $\pi(R(t))^{2}$ times the thermal penetration depth $\delta_{t h} \propto \sqrt{\kappa}$, with $\kappa=k /\left(\rho c_{p}\right)$ the thermal diffusivity of the liquid. The number of growing crystals is then described by:

$$
n_{\text {crystals }} \propto J_{0} \int_{0}^{t / \tau_{c}} R^{2} \delta_{\text {th }} \mathrm{d} t .
$$

In our experiments, the wetting dynamics of a droplet is indistinguishable from the iso-thermal spreading, as found in Refs. [32-35]. The wetting follows the spreading law $R / R_{0} \propto\left(t / \tau_{c}\right)^{1 / 2}$ until the contact line suddenly stops advancing due to the solidification, see Section 2.E. To close the problem, we assume that the early-time spreading dynamics of the droplet remains unaffected by the nucleation. Combining this spreading law with equation (2.1), we derive $n_{\text {crystals }} \propto\left(t / \tau_{c}\right)^{5 / 2}$, which is consistent with the experimental data (figure 2.3). Note that for very small $\Delta T$ (orange circles in figure 2.3) the amount of crystals does not follow this power law. Due to the low surface energy, $\tau_{g}$ may even be larger than $\tau_{c}$. Surface impurities can lead to random nucleation in this case.

We are now in a position to identify the mechanism that leads to the sudden arrest of the moving contact line. Our experiments reveal that the local nature of the interactions between growing crystals and the moving contact line is one reason that leads to its arrest. Figure 2.4(a) highlights two distinct events that exemplify the physical mechanism responsible for this contact-line arrest. In the case that a crystal nucleates at the contact line (figure 2.4(a), centre panel), it immediately arrests the advancing motion locally. This random event at the moving contact line is caused by local heterogeneities on the substrate. In contrast, a crystal nucleating far away from the contact line (figure 2.4(a), left panel) does not affect its motion immediately, but if its growth catches up with the advancing contact line, it locally arrests the spreading (figure 2.4(a), right panel). Consequently, in both cases the droplet footprint evolves non-axi-symmetrically.

The motion of the contact line was hypothesised to arrest when the advancing velocity of the contact line becomes equal to the crystal growth speed for the applied under-cooling. This hypothesis leads to a scaling law for the arrest radius $R_{\text {end }}$ [22]:

$$
R_{\text {end }} / R_{0} \propto R_{0} /\left(\tau_{c} U_{g}\right),
$$

with $U_{g}$ the crystal growth speed, which was approximated as $U_{g}=\beta \Delta T$ [29], where the kinetic undercooling coefficient $\beta$ (with units $\mathrm{m} \mathrm{s}^{-1} \mathrm{~K}^{-1}$ ) is a fitting parameter. Here, we directly obtain the crystal growth speed, and with that the undercooling coefficient $\beta$, by measuring the temporal growth of several crystals after their nucleation at the droplet-substrate contact area for different substrate undercooling. Note that $T_{c}$ is the natural choice instead of the substrate temperature $T_{s}$ for this undercooling, as the 
(a)

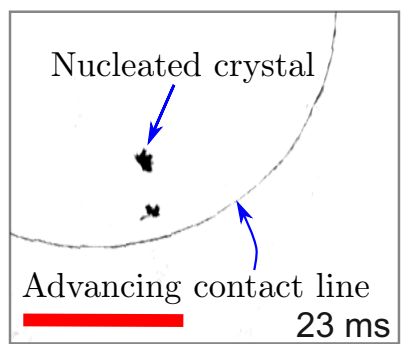

(b)

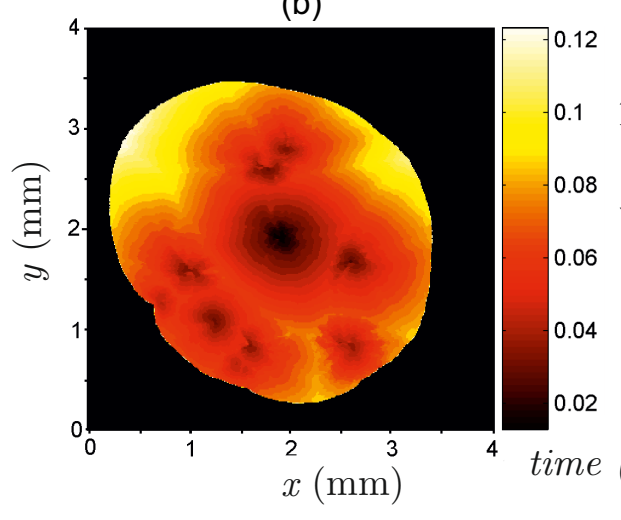

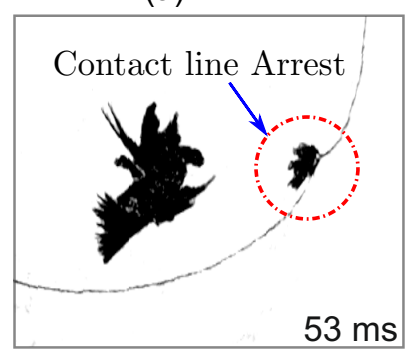

$53 \mathrm{~ms}$

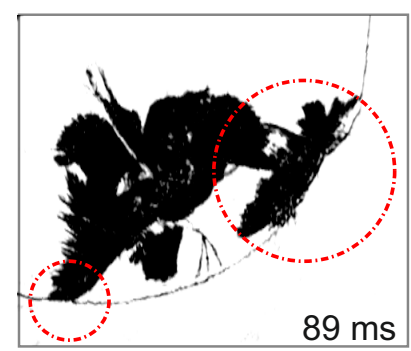

(c)

Figure 2.4: Contact line arrest and crystal growth: (a) Nucleation near the contact line, leading to local contact-line arrest (red circles), the red bar indicates a length of $1 \mathrm{~mm}, \Delta T=1.0 \mathrm{~K}$. (Left panel) Nucleation far away from the contact line. (Center panel) Nucleation close to the contact line leads to contact-line arrest. (Right Panel) Crystals growing towards the contact line lead to contact line arrest. (b) Footprint of a solidified droplet with the temporal evolution (color variation) of solidification during drop spreading starting from sequentially formed isolated crystals, $\Delta T=3.4 \mathrm{~K}$. (c) Growth velocity as a function of the undercooling $\Delta T$. The error bars indicate the minimal and maximal growth velocity at a certain temperature. The dashed line shows the growth velocity $U_{\mathrm{g}}=\beta \Delta T$, with $\beta \approx 4.5 \mathrm{~mm} \mathrm{~s}^{-1} \mathrm{~K}^{-1}$.

solidification is dictated by the contact temperature. Figure $2.4 \mathrm{~b}$ shows a typical evolution of crystals after nucleation in the wetted area. The crystal growth speed shows a linear dependence on $\Delta T$ (figure $2.4 \mathrm{c}$ ), resulting in $\beta \approx 4.5 \mathrm{~mm} \mathrm{~s}^{-1} \mathrm{~K}^{-1}$. This value is slightly smaller than that for hexadecane spreading on copper obtained from a fit of the data in Ref. [22]. With this independently measured kinetic cooling coefficient, we can now directly test the prediction for contact line arrest, equation (2), by comparing to our measurements. A very good agreement is found, using a prefactor of 0.18 , with a relative error within $20 \%$ for most data (see Section2.E). However, our experimental results significantly deviate from the model at low undercooling $\Delta T<3 \mathrm{~K}$. We believe that the implicit assumptions (infinitely small lag time $\tau_{g} \sim 0$ and high probability of nucleation sites at the contact line) made in the model for droplets spreading on 


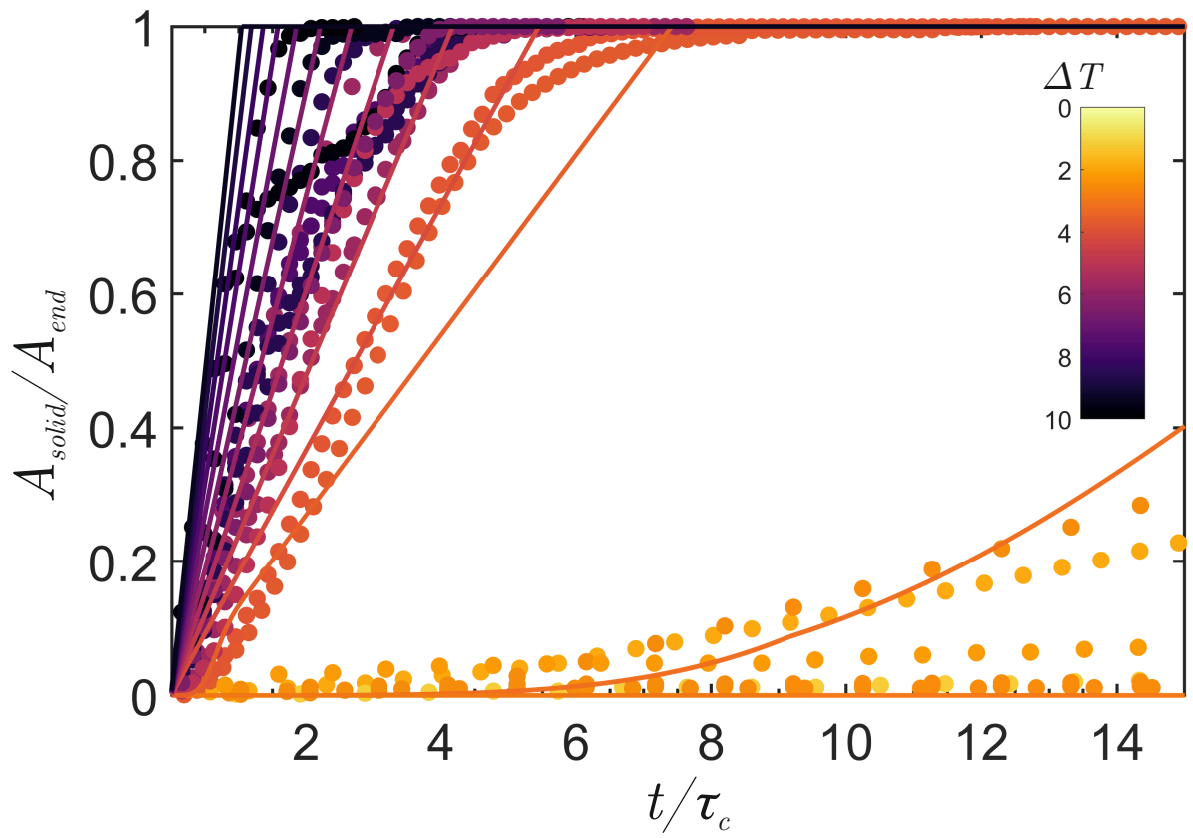

Figure 2.5: Time dependence of the fraction of solidified material at the substrate for various temperatures. The solid lines show equation (2.3), for $1 \mathrm{~K}<\Delta T<8 \mathrm{~K}$, see color code. A large change of solidification behaviour is seen for the model around $\Delta T=2.7 \mathrm{~K}$. Note that data with the same color can come from different experiments.

substrates are not applicable at low $\Delta T$. Conversely, for the complete range of $\Delta T$ we do not observe any preference of nucleation sites near the contact line. Modelling the contact line arrest at small undercooling requires to properly account for the statistical nature of the occurence of nucleations sites on the substrate, which is beyond the scope of this work.

Finally, we shift our focus to the temporal growth of the solidified area along the droplet-substrate interface. We follow the formulation proposed in Ref. [36, 37] for heterogeneous nucleation and growth of the solidified phase on an undercooled surface. It assumes time-independent growth velocity $U_{g}$ and nucleation rate $J_{0}$, with sites equally distributed over the substrate. We use the Johnson-Mehl-Avrami-Kolmogorov $(J M A K)$ equation to determine the $2 \mathrm{D}$ growth of the solidified surface fraction as a function of time as: $\chi=1-\exp \left(-4 \pi N_{0} U_{g}^{2} t^{2}\right)$. The amount of crystals per area is estimated as $N_{0}=\int_{0}^{t} J_{0} \delta_{t h} d t=2 / 3 J_{0} \delta_{t h} t$. As the droplet continues to spread over the substrate, the area available for nucleation increases. Hence, we rescale the JMAK 


\subsection{Conclusions}

equation by the instantaneous wetted area $(R(t))^{2} / R_{\text {end }}^{2}$, to find:

$$
\frac{A_{\text {solid }}}{A_{\text {end }}}=\left(1-\exp \left(-\frac{8 \pi}{3} J_{0} \delta_{t h} U_{g}^{2} t^{3}\right)\right)\left(\frac{R(t)}{R_{\text {end }}}\right)^{2},
$$

where we use the arrest criterion (equation (2.2)) to obtain $R_{\text {end }}$.

The evolution of the solidified area fraction for various surface undercoolings is shown in figure 2.5 . For $\Delta T<2.5 \mathrm{~K}$, the slow nucleation leads to a very slow increase in solidified area fraction. At higher $\Delta T$, both the nucleation rate and crystal growth speed increase. Consequently, the solidified area fraction grows faster. For even higher under-cooling $\Delta T>4.4 \mathrm{~K}$, the solidified fraction growth rate matches the spreading of the droplet, thus: $A_{\text {solid }} / A_{\text {end }} \approx\left(R(t) / R_{\text {end }}\right)^{2}$. We find that equation (2.3) agrees with the experimental data over a wide range of $\Delta T$. The only adjustable parameter is the geometrical factor $f\left(\theta_{l s}\right)$, since the average nucleus contact angle is not directly measurable [38]. From a fit to the data, we find $f\left(\theta_{l s}\right) \approx 0.12$, which implies an average nucleus contact angle of $\theta_{l s} \approx 55^{\circ}$. It must be pointed out that for undercooling below $2.5 \mathrm{~K}$, the model does not predict any solidification within the typical timescale of experiments. This observation corroborates our conjecture that it is impurities that cause nucleation and growth in this temperature range.

\subsection{Conclusions}

In summary, we directly visualized the surface solidification during spreading of hexadecane droplets on an under-cooled sapphire surface, using high-speed TIR imaging. Two distinct solidification behaviours are observed, which are explained by classical nucleation theory. The number of crystals in a spreading droplet scale with

$n_{\text {crystals }} \propto\left(t / \tau_{c}\right)^{5 / 2}$. Furthermore, we reveal that the arrest velocity is approximately equal to the crystal growth velocity determined by the undercooling. This direct observation is in line with the model developed in Ref. [22]. However, it is not valid for very weak under-cooling, since the nucleation happens randomly over the surface. Apart from the processes near the contact line, we reveal that the crystal growth speed directly depends on the contact temperature $T_{c}$, rather than on the initial temperature of the substrate $T_{s}$. Finally, we showed that the 2-dimensional $J M A K$ equation, rescaled for the time dependent contact area accurately predicts the temporal growth of the solidified area fraction of a spreading droplet. Our results give insight into both local and overall solidification processes near the contact line and the substrate. The visualization method opens a new experimental pathway of elucidating solidification behavior near substrates on a time-resolved macro-and microscopic scale. This technique can be used to directly measure the early solidification behaviour for many relevant applications in manufacturing. 


\section{A Experimental setup}

The experimental setup is depicted in figure 2.1. A hexadecane droplet $\left(\mathrm{C}_{16} \mathrm{H}_{34}\right.$, SIGMA-ALDRICH, thermal properties in table 2.1), with freezing point $T_{f}=18^{\circ} \mathrm{C}$, is formed at the end of a needle (NORDSON EFD, with inner diameter $d_{i}=0.33$ $\mathrm{mm}$ ). This needle is fed by a syringe pump (HARVARD PHD2000), resulting in a droplet diameter of $D_{0}=1.7 \pm 0.1 \mathrm{~mm}$. The hexadecane is slightly heated to an initial droplet temperature of $T_{0}=37^{\circ} \mathrm{C}$ to prevent solidification in the needle. This heating has no significant influence on the spreading behavior [22]. The droplet is gently deposited onto a sapphire prism (CRYSTAN LTD.), which is cooled below the freezing temperature of the droplet. The thermal properties of the prism are given in table 2.1 The prism is placed in an aluminum holder, that is PID-controlled at a fixed temperature ranging from $8{ }^{\circ} \mathrm{C}$ to $20^{\circ} \mathrm{C}$. Before each spreading event the substrate temperature of the sapphire prism is measured with an NTC-type thermistor (WAVELENGTH ElECTRONICS). We record the bottom view with a high-speed camera (Photron FastCam SA-X2) equipped with a long distance microscope (Navitar 12x Telecentric zoom system) at recording rates from $20000 \mathrm{fps}$ to $30000 \mathrm{fps}$ and a spatial resolution of $9 \mu \mathrm{mpixel}^{-1}$. In order to discriminate between the solidified, wetted and dry areas we employ TIR imaging [26]. The substrate is illuminated under an angle $\theta$ by a $60 \mathrm{~mW}$ laser (wavelength $643 \mathrm{~nm}$ ) expanded to about $20 \mathrm{~mm}$ diameter and introduced into the prism via mirrors.

\section{B Material properties}

Table 2.1: Material properties of the liquid hexadecane and sapphire prism

\begin{tabular}{lll}
\hline Material & $\begin{array}{l}\text { Hexadecane } \\
(\text { droplet) }\end{array}$ & $\begin{array}{l}\text { Sapphire } \\
\text { (prism) }\end{array}$ \\
\hline Density $\rho\left(\right.$ kilogram $\left./ \mathrm{m}^{3}\right)$ & 770 & 3980 \\
Thermal Conductivity k $(\mathrm{watt} / \mathrm{m} / \mathrm{K})$ & 0.15 & 34.6 \\
Heat capacity $c_{p}($ joule $/ \mathrm{kg} / \mathrm{K})$ & 2310 & 761 \\
Surface tension $\sigma($ newton $/ \mathrm{m})$ & 0.028 & - \\
Viscosity $\eta$ (pascals) & 0.003 & - \\
\hline
\end{tabular}

\section{C Visualisation}

Here we explain more details of the TIR imaging method employed to visualize the solidification processes. This method has the advantage to be only sensitive to measure processes in a thickness smaller than the first micrometer of the droplet. Illuminating 


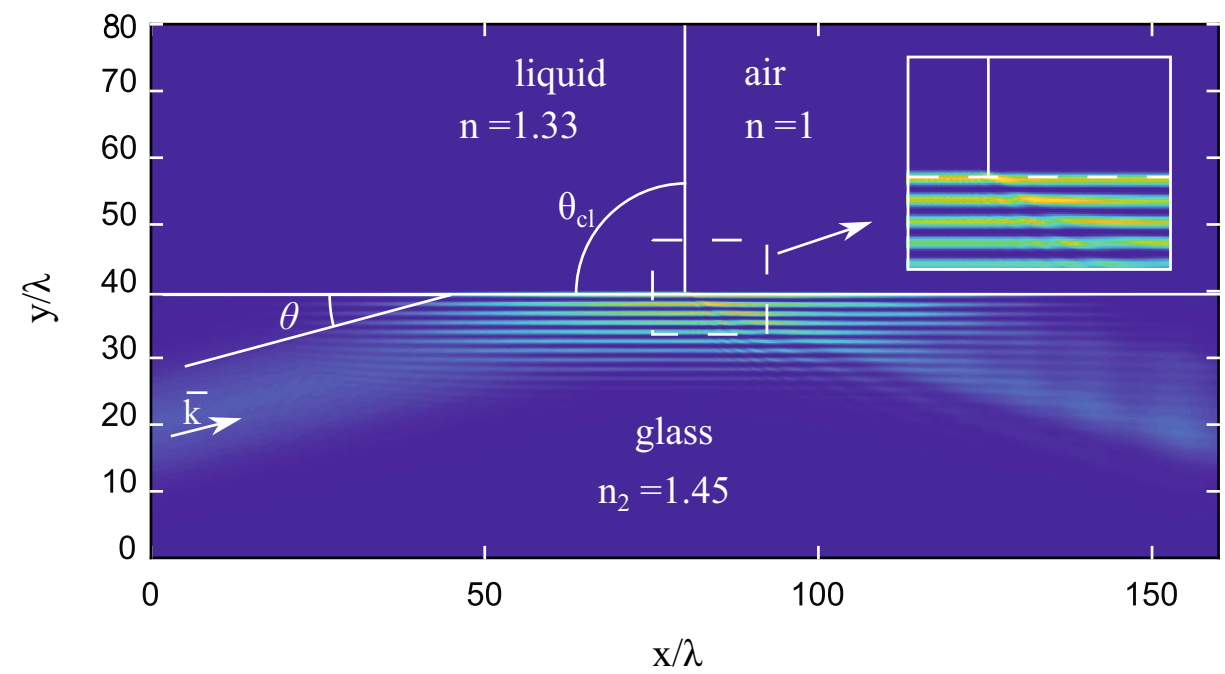

Figure 2.6: Simulation results, showing the normalized intensity field of a collimated laser beam of width $10 \lambda$. The wave vector $\vec{k}$, which determines the incident angle $\theta$ with the glass surface, is chosen such that the light undergoes total internal reflection for both the liquid (left) and air (right) phases. The vertical white line represents the liquid-air interface, whereas the inset reveals the details at the contact line, showing the diffraction patterns leading to the visibility of the contact line.

the bottom of the prism is achieved by using a monochromatic light source. We use a $643 \mathrm{~nm}$ diode laser, which is expanded by a set of lenses, to have the complete footprint of the drop illuminated. The camera records the reflected light through the other side of the prism.

The incident angle $\theta$ should be larger than the critical angle $\theta_{c}=\sin ^{-1}\left(n_{2} / n_{1}\right)$ for the TIR imaging to work. Here $n_{2}$ is the refractive index of the sapphire prism and $n_{1}$ that of the medium across the interface. The image of the droplet is distorted to an ellipse, back transformation and angles of incidence are obtained as described in Ref. [26]. In this Letter, only reconstructed images are shown. In earlier work, for heated surfaces [39], $\alpha$ was chosen to be larger than $\theta_{c}$, using $n_{1}=1$ for air, but smaller than $n_{2}$ for the liquid. This results in TIR for locations that are dry, resulting in a white spot on the camera and normal refraction according to the Fresnel equations for the wetted areas, resulting in the loss of intensity on the camera.

In the present study however, we operate the setup for $\alpha$ being smaller than $\theta_{c}$ for both air and the liquid. As a consequence light is refracted everywhere, except where the refractive index makes a sudden jump. This jump can both come from the sudden change from air to liquid or from fluctuations in the refractive index as a result of amorphous solid patches. Both cases result in diffraction of the evanescent wave on 
the other side of the prism interface. It is this diffraction which enables us to observe the moving contact line as well as the solidified areas in the drop.

A simulation of a contact line is shown in figure 2.6, where a Gaussian beam is propagating from right to left and is reflected on the prism surface. The simulations solve the time independent Helmholtz-equation in the three media, using the code developed by Osnabrugge et. al. [40]. The domain is normalized by the incident wave length $\lambda$ and the beam width is $10 \lambda$. The prism material has a refractive index of $n=1.45$; and $n=1$ is used on the right, for the air, whereas $n=1.33$ is used for the liquid part. The resulting normalized electric field is represented by the color between zero (blue) and 1 (yellow). The inset magnifies the details at the contact line. The difference in decay length $d=\lambda_{0} /\left(4 \pi n_{1}\right)\left(\sin ^{2} \theta-\left(n_{2} / n_{1}\right)^{2}\right)^{-1 / 2}$ for the glass-air and glass-liquid interfaces results in the intensity fluctuations in the right part of the glass domain. However, the finite aperture of optical systems used in experiments cannot resolve this small scale feature. The diffraction pattern captured is used to identify the contact line position.

\section{D Nucleation rate}

The nucleation rate can be expressed as an Arrhenius type reaction velocity:

$$
J_{0}=A \exp \left\{\frac{-E_{a}}{k_{B} T_{f}}\right\}
$$

where $J_{0}$ is the nucleation rate, $k_{B}$ the Boltzmann constant, and $A$ the attempt frequency. $E_{a}$ is a critical activation energy to form a stable nucleus. The formation of a stable crystalline phase in an under-cooled liquid is controlled by the local free energy barrier. The activation energy associated with nucleation, assuming the nucleus is a sphere, is given by $E_{a}=(16 \pi / 3) \gamma_{l s}^{3} f\left(\theta_{l s}\right) /(\Delta g)^{2}$, where $\Delta g=\Delta S_{f u s} \Delta T$ is the free energy difference between the liquid and solid phase and $\gamma_{l s}=0.0068 \mathrm{~J} \mathrm{~m}^{-2}$ [41] is the interfacial tension between the two phases. The entropy of fusion is $\Delta S_{f u s}=$ $6.28 \cdot 10^{5} \mathrm{~J} \mathrm{~m}^{-3} \mathrm{~K}^{-1}$ [31]. For a spherical particle on a foreign surface, $f\left(\theta_{l s}\right)=$ $1 / 4\left(2+\cos \theta_{l s}\right)\left(1-\cos \theta_{l s}\right)^{2}$ is the geometrical correction factor, where $\theta_{l s}$ is the contact angle between a solid nucleus and the liquid phase surrounding it with the substrate [30]. This correction factor is determined by the change in volume and surface area between a spherical nucleus and a spherical cap on a surface. It expresses the difference in the energy barrier when heterogeneous nucleation takes place, which occurs for sessile droplets [42]. The nucleation behavior of spreading droplets is not yet known, as far as the authors are aware. The attempt frequency $A$ is proportional to the characteristic frequency of molecular motion $[31,43,44], A=C \sqrt{\frac{\gamma_{l s}}{k_{B} T_{p r i s m}}} \alpha \frac{\mathscr{D}}{d^{2}}$. Here $\mathrm{C}$ is a numerical constant set to 1.65 [45], $\alpha, d$ and $\mathscr{D}=\left(k_{B} T_{\text {prism }}\right) /(3 \pi \eta d)$ are 
the molecular number density, molecular diameter and the Stokes-Einstein diffusion constant of hexadecane in its liquid phase.

\section{E Droplet spreading}

Here we show the extent of hexadecane spreading on a sapphire substrate, including the maximum spreading radius. Side view images are obtained using a high speed camera (FASTCAM-APX RS) fitted with a macro lens at $10000 \mathrm{fps}$, with a spatial resolution of $18 \mu \mathrm{mpixel}^{-1}$, where the initial frame is synchronized with the bottom view. For substrate temperatures above the freezing temperature, the isothermal spreading laws are recovered, figure 2.7, as was reported for the spreading of hexadecane droplets on copper [22]. Initially,

$$
\frac{R}{R_{0}} \propto\left(t / \tau_{c}\right)^{1 / 2},
$$

with the spreading radius $R(t)$, droplet radius $R_{0}$, and the capillary time $\tau_{c}=\left(\rho R_{0}^{3} / \sigma\right)^{1 / 2}$ $[32-34,46]$. At later stages, the spreading resembles Tanner's law $R / R_{0} \propto\left(\sigma t / \eta R_{0}\right)^{1 / 10}$ for the spreading of a small viscous drop [47]. For $T<T_{\mathrm{f}}$, the contact line is arrested due to solidification. Figure $2.7 \mathrm{~b}$ shows the mean arrest radius $R_{\text {end }}$ as a function of the under-cooling. For $\Delta T>3.5 \mathrm{~K}$, they follow the criterium of Ref.[22]:

$$
\frac{R_{\text {end }}}{R_{0}} \propto \frac{R_{0}}{\tau_{c} \beta \Delta T},
$$

where $\beta$ is the kinetic under-cooling coefficient. The best fit to this equation for our data was found with a prefactor of 0.18 . This leads to a typical error $\left|\left(R_{t h}-R_{\exp }\right) / R_{t h}\right|<$ $20 \%$, the theoretical radius $R_{t h}$ and the measured radius $R_{\exp }$ for undercooling $\Delta T>$ $3 \mathrm{~K}$, see the inset in figure $2.7 \mathrm{~b}$. Nucleation at the contact line is not distributed evenly, therefore the arrested droplets are not constant in radius, which is shown by the error bars in figure $2.7 \mathrm{~b}$. 

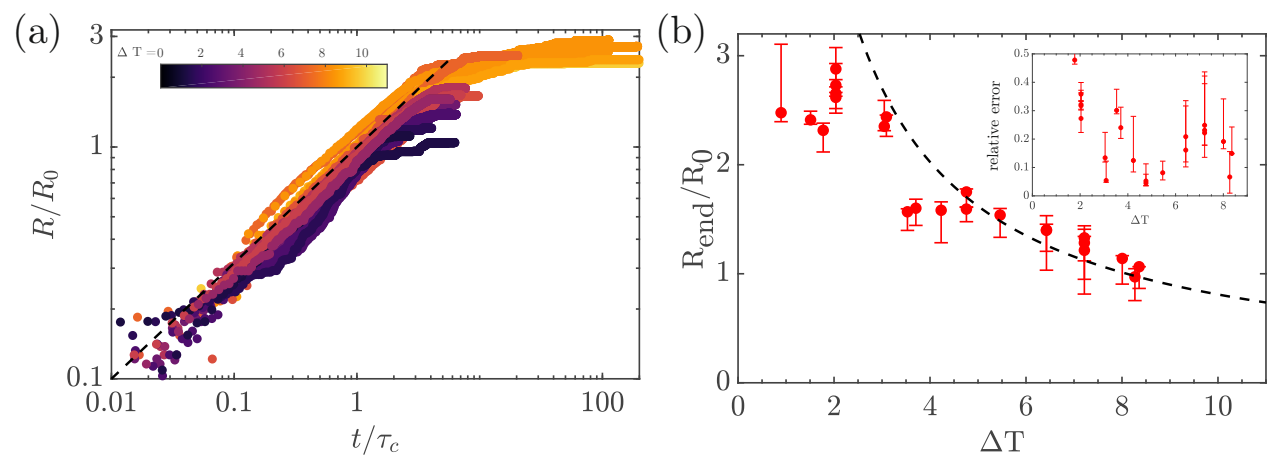

Figure 2.7: (a) Spreading radius versus time for a range of substrate temperatures, with — the inertial scaling $\propto t^{1 / 2}$, Equation (2.5). (b) Radius of arrest versus temperature. - - -is the model of equation (2.6). The error bars show the minimum and maximum radius of a single arrest event. For $\Delta T<3 \mathrm{~K}$, the model is not valid. The inset shows the relative error $\left|\left(R_{t h}-R_{\text {exp }}\right) / R_{t h}\right|$

\section{References}

[1] H. Chen, Y. Wu, H. Xia, B. Jing, and Q. Zhang, "Review of ice-pavement adhesion study and development of hydrophobic surface in pavement deicing", J. Traffic Transp. Eng. (English Ed. 5, 224-238 (2018).

[2] T. Cebeci and F. Kafyeke, "Aircraft Icing”, Annu. Rev. Fluid Mech. 35, 11-21 (2002).

[3] M. Farzaneh, Atmospheric Icing of Power, volume 84 (Springer Science Business Media B.V, Chicoutimi) (2008).

[4] D. J. Hayes, D. B. Wallace, and W. Royall Cox, "MicroJet printing of solder and polymers for multi-chip modules and chip-scale packages", in Proceedings-SPIE Int. Soc. Opt. Eng., 242-247 (1999).

[5] D. Attinger, Z. Zhao, and D. Poulikakos, “An Experimental Study of Molten Microdroplet Surface Deposition and Solidification: Transient Behavior and Wetting Angle Dynamics”, J. Heat Transfer 122, 544-556 (2002).

[6] S. Chandra and P. Fauchais, "Formation of solid splats during thermal spray deposition", J. Therm. Spray Technol. 18, 148-180 (2009).

[7] M. Vaezi, H. Seitz, and S. Yang, "A review on 3D micro-additive manufacturing technologies”, Int. J. Adv. Manuf. Technol. 67, 1721-1754 (2013). 
[8] C. Visser, R. Pohl, C. Sun, G. Römer, B. Huis In 'T Veld, and D. Lohse, “Toward 3D Printing of Pure Metals by Laser-Induced Forward Transfer", Adv. Mater. 27, 4087-4092 (2015).

[9] O. R. Enríquez, Á. G. Marín, K. G. Winkels, and J. H. Snoeijer, "Freezing singularities in water drops", Phys. Fluids 24, 091102 (2012).

[10] M. Pasandideh-Fard, V. Pershin, S. Chandra, and J. Mostaghimi, "Splat shapes in a thermal spray coating process: Simulations and experiments", J. Therm. Spray Technol. 11, 206-217 (2002).

[11] A. I. Fedorchenko and A. B. Wang, "Non-equilibrium solidification of the molten metal droplets impacting on a solid surface", Int. J. Heat Mass Transf. 50, 24632468 (2007).

[12] W. Kong and H. Liu, "A theory on the icing evolution of supercooled water near solid substrate”, Int. J. Heat Mass Transf. 91, 1217-1236 (2015).

[13] E. Ghabache, C. Josserand, and T. Séon, "Frozen impacted drop: from fragmentation to hierarchical crack patterns", Phys. Rev. Lett. 117, 074501 (2016).

[14] V. Thiévenaz, T. Séon, and C. Josserand, "Solidification dynamics of an impacted drop”, J. Fluid Mech. 874, 756-773 (2019).

[15] M. V. Gielen, R. de Ruiter, R. B. Koldeweij, D. Lohse, J. H. Snoeijer, and H. Gelderblom, "Solidification of liquid metal drops during impact", J. Fluid Mech. 883, A32 (2020).

[16] P. Kant, R. B. Koldeweij, K. Harth, M. A. van Limbeek, and D. Lohse, "Fastfreezing kinetics inside a droplet impacting on a cold surface", PNAS 117, 2788-2794 (2020).

[17] C. Gurganus, A. B. Kostinski, and R. A. Shaw, "Fast imaging of freezing drops: No preference for nucleation at the contact line", J. Phys. Chem. Lett. 2, 14491454 (2011).

[18] C. Tropea, M. Schremb, and I. V. Roisman, "Physics of SLD Impact and Solidification", in 7th Eur. Conf. Aeronaut. Aerosp. Sci., EUCASS2017-512 7TH (2017).

[19] C. Gurganus, A. B. Kostinski, and R. A. Shaw, "High-speed imaging of freezing drops: Still no preference for the contact line”, J. Phys. Chem. C 117, 6195-6200 (2013). 
[20] S. Schiaffino and A. A. Sonin, "On the theory for the arrest of an advancing molten contact line on a cold solid of the same material", Phys. Fluids 9, 22272233 (1997).

[21] H. Jones, "Cooling, freezing and substrate impact of droplets formed by rotary atomization", J. Phys. D. Appl. Phys. 4, 1657-1660 (1971).

[22] R. de Ruiter, P. Colinet, P. Brunet, J. H. Snoeijer, and H. Gelderblom, "Contact line arrest in solidifying spreading drops", Phys. Rev. Fluids 2, 043602 (2017).

[23] J. Kim, "Spray cooling heat transfer: The state of the art", Int. J. Heat Fluid Flow 28, 753-767 (2007).

[24] J. M. Kolinski, S. M. Rubinstein, S. Mandre, M. P. Brenner, D. A. Weitz, and L. Mahadevan, "Skating on a film of air: drops impacting on a surface", Phys. Rev. Lett. 108, 074503 (2012).

[25] M. Khavari, C. Sun, D. Lohse, and T. Tran, "Fingering patterns during droplet impact on heated surfaces", Soft Matter 11, 3298-3303 (2015).

[26] M. Shirota, M. A. van Limbeek, D. Lohse, and C. Sun, "Measuring thin films using quantitative frustrated total internal reflection (FTIR)", Eur. Phys. J. E 40, 54 (2017).

[27] H. S. Carslaw and J. C. Jaeger, Conduction of heat in solids, volume 2 (Oxford Science Publications, Oxford) (2016).

[28] O. Söhnel and J. W. Mullin, "Interpretation of crystallization induction periods", J. Colloid 123, 43-50 (1988).

[29] S. H. Davis, Theory of solidification (Cambridge University Press) (2001).

[30] J. Mullin, Crystallisation, volume 6, 4th edition (Butterworth-Heinemann, Oxford) (2007).

[31] A. B. Herhold, D. Ertaş, A. J. Levine, and H. E. King, "Impurity mediated nucleation in hexadecane-in-water emulsions", Phys. Rev. E 59, 6946 (1999).

[32] A. L. Biance, C. Clanet, and D. Quéré, "First steps in the spreading of a liquid droplet”, Phys. Rev. E 69, 016301 (2004).

[33] J. C. Bird, S. Mandre, and H. A. Stone, "Short-time dynamics of partial wetting", Phys. Rev. Lett. 100, 234501 (2008).

[34] K. G. Winkels, J. H. Weijs, A. Eddi, and J. H. Snoeijer, "Initial spreading of low-viscosity drops on partially wetting surfaces", Phys. Rev. E 85, 055301(R) (2012). 
[35] S. Wildeman, C. W. Visser, C. Sun, and D. Lohse, "On the spreading of impacting drops", J. Fluid Mech. 805, 636-655 (2016).

[36] E. Dutra Zanotto, "Surface nucleation in a diopside glass", J. Non. Cryst. Solids 130, 217-219 (1991).

[37] M. Fanfoni and M. Tomellini, "The Johnson-Mehl-Avrami-Kolmogorov model: A brief review", Nuovo Cim. della Soc. Ital. di Fis. D - Condens. Matter, At. Mol. Chem. Physics, Biophys. 20, 1171-1182 (1998).

[38] A. Määttänen and M. Douspis, "Estimating the variability of contact parameter temperature dependence with the Monte Carlo Markov Chain method", GeoResJ 4, 46-55 (2014).

[39] M. A. Van Limbeek, M. Shirota, P. Sleutel, C. Sun, A. Prosperetti, and D. Lohse, "Vapour cooling of poorly conducting hot substrates increases the dynamic Leidenfrost temperature", Int. J. Heat Mass Transf. 97, 101-109 (2016).

[40] G. Osnabrugge, S. Leedumrongwatthanakun, and I. M. Vellekoop, "A convergent Born series for solving the inhomogeneous Helmholtz equation in arbitrarily large media”, J. Comput. Phys. 322, 113-124 (2016).

[41] M. J. Oliver and P. D. Calvert, "Homogeneous nucleation of n-alkanes measured by differential scanning calorimetry", J. Cryst. Growth 30, 343-351 (1975).

[42] M. Dorrestijn, S. Jung, C. M. Megaridis, D. Raps, A. Das, and D. Poulikakos, “Are Superhydrophobic Surfaces Best for Icephobicity?", Langmuir 27, 30593066 (2011).

[43] D. T. Wu, "Nucleation Theory”, Solid State Phys. 50, 37-187 (1996).

[44] A. B. Herhold, H. E. King, and E. B. Sirota, "A vanishing nucleation barrier for the n-alkane rotator-to-crystal transformation", J. Chem. Phys. 116, 9036-9050 (2002).

[45] K. F. Kelton, A. L. Greer, and C. V. Thompson, "Transient nucleation in condensed systems”, J. Chem. Phys. 79, 6261-6276 (1983).

[46] B. B. Stapelbroek, H. P. Jansen, E. S. Kooij, J. H. Snoeijer, and A. Eddi, "Universal spreading of water drops on complex surfaces", Soft Matter 10, 2641-2648 (2014).

[47] L. H. Tanner, "The Spreading of Silicone Oil Drops on Horizontal Surfaces", J. Phys. D 12, 1473-1484 (1979). 



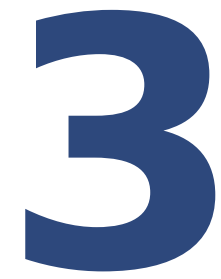

\section{Fast-freezing kinetics inside a droplet impacting on a cold surface *}

Freezing or solidification of impacting droplets is omnipresent in nature and technology, be it a rain droplet falling on a supercooled surface; in inkjet printing, where often molten wax is used; in additive manufacturing or metal-production processes; or in extreme ultraviolet lithography (EUV) for the chip production, where molten tin is used to generate the EUV radiation. For many of these industrial applications, a detailed understanding of the solidification process is essential. Here, by adopting an optical technique in the context of freezing - namely, total internal reflection (TIR)—we elucidate the freezing kinetics during the solidification of a droplet while it impacts on an undercooled surface. We show that at sufficiently high undercooling, a peculiar freezing morphology exists that involves sequential advection of frozen fronts from the center of the droplet to its boundaries. This phenomenon is examined by combining elements of classical nucleation theory to the large-scale hydrodynamics on the droplet scale, bringing together two subfields which traditionally have been quite separated. Furthermore, we report a self-peeling phenomenon of a frozen splat that is driven by the existence of a transient crystalline state during solidification.

*Published as: "Fast-freezing kinetics inside a droplet impacting on a cold surface" P. Kant, R.B.J. Koldeweij, K. Harth, M.A.J. van Limbeek, D. Lohse, Proceedings of the National Academy of Sciences, 117(6), 2788-2794 (2020) 


\subsection{Introduction}

The impact of a droplet on an undercooled solid surface instigates a number of physical processes simultaneously, including drop scale fluid motion, heat transfer between the liquid and the substrate, and the related phase transition. Whereas a large number of studies have investigated the corresponding interface deformations and the spreading of a droplet after it impinges onto an undercooled surface [1-9], the kinetics of phase transition within the impacting droplet has been addressed only in a few [10-12]. Moreover, among the studies concerning solidification kinetics, only the regimes where phase-transition effects are slower than the fast dynamics of droplet impact have been investigated. Here, we explore freezing behaviors that arise due to the rapid solidification of an impacting droplet at a sufficiently high substrate undercooling. Such scenarios are encountered in a number of industrial processes ranging from additive manufacturing $[13,14]$ to thermal plasma spraying of ceramics and metallic materials [15-17], extreme ultraviolet lithography [18, 19], etc.

In this chapter, we adapt the total-internal-reflection (TIR) technique [20-22] to visualize the phase transition in the vicinity of the liquid-substrate interface after a droplet impacts onto an undercooled transparent surface. This unique technique allows temporally and spatially resolved insight into the nucleation events and crystal growth occurring next to the cold surface on an evanescent length scale $(\sim 100 \mathrm{~nm})$, which is otherwise inaccessible through any other optical technique. Moreover, it allows us to monitor the delamination of the frozen splat from the substrate at later time. The details of the experimental setup are provided in Materials and Methods.

\subsection{Results and Discussion}

The sequences of experimental snapshots in Fig. 3.1(a-d) highlight different freezing morphologies of a liquid hexadecane droplet after it impinges on a flat cold surface at various undercooling $\Delta T=T_{\mathrm{m}}-T_{\mathrm{s}} ; T_{\mathrm{m}}=18^{\circ} \mathrm{C}$ is the melting temperature of the liquid and $T_{\mathrm{s}}$ is the temperature of the substrate. In all the cases, a droplet of diameter $D=1.58 \pm 0.02 \mathrm{~mm}$ at ambient temperature $T_{\mathrm{d}} \approx 20^{\circ} \mathrm{C}$ impacts onto the horizontal surface of an undercooled sapphire prism with a velocity $U \simeq 2.8 \mathrm{~m} / \mathrm{s}$. The Weber number We $=\rho D U^{2} / \sigma$ and Reynolds numbers $\mathrm{Re}=\rho D U / \mu$ associated with this impact are 340 and 1150, respectively; $\rho$ is the density, $\sigma$ is the surface tension and $\mu$ is the dynamic viscosity of the liquid. Typical deformations of the droplet interface, recorded from side-view, associated with these impact parameters are shown in the sequence Fig. 3.1e. Note that, since hexadecane highly wets the sapphire surface, with static equilibrium contact angle $\theta_{\mathrm{e}} \approx 15^{\circ}$, no receding or rebound motion of droplets was observed after the impact.

Immediately after a droplet makes contact with the flat substrate, two concentric 
(a) $\Delta T=2 \mathrm{~K}$

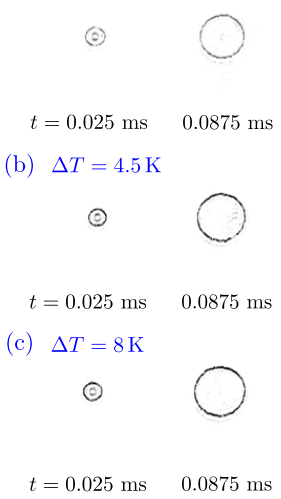

(d) $\Delta T=11 \mathrm{~K}$


$0.1125 \mathrm{~ms}$

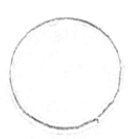

$0.5125 \mathrm{~ms}$

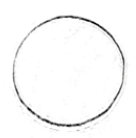

$0.5125 \mathrm{~ms}$

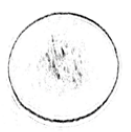

$0.5125 \mathrm{~ms}$
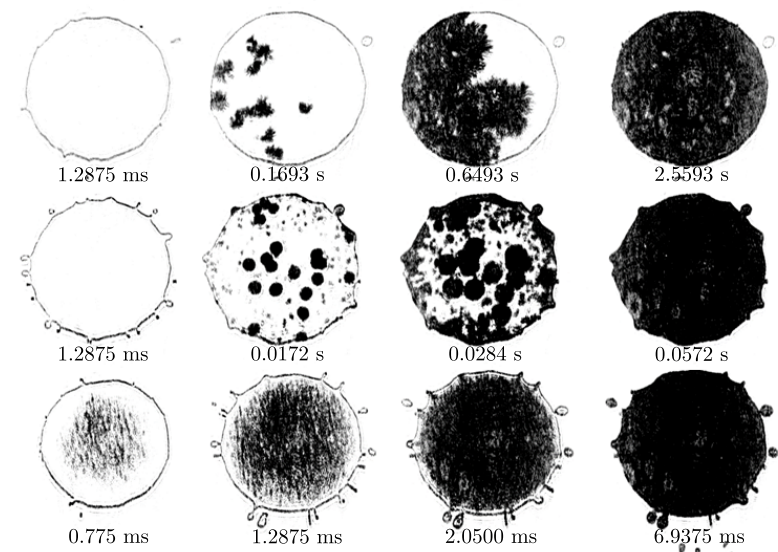

$0.775 \mathrm{~ms}$
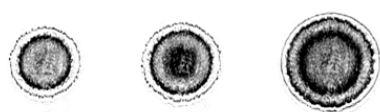

$0.1875 \mathrm{~ms}$

$0.2625 \mathrm{~ms}$

$0.4125 \mathrm{~ms}$

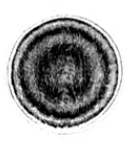

$0.6500 \mathrm{~ms}$
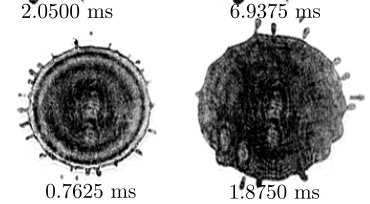

Figure 3.1: (a-d) Sequences of experimental snapshots highlighting the effect of substrate undercooling $\Delta T=T_{\mathrm{m}}-T_{\mathrm{s}}=$ (a) $2 \mathrm{~K}$ (b) $4.5 \mathrm{~K}$ (c) $8 \mathrm{~K}$ (d) $11 \mathrm{~K}$ on the freezing morphology of an impacting droplet. In all the cases the velocity and the temperature of the droplet at the time of impact are $U=2.8 \mathrm{~m} / \mathrm{s}$ and $T_{\mathrm{d}}=20^{\circ} \mathrm{C}$, respectively. (e) Typical interface deformations associated with the impact of a droplet of size $D=1.58 \mathrm{~mm}$ on an undercooled surface; recorded in side view at $14000 \mathrm{fps}$.

rings appear in the TIR images: the first panel of each sequence in Fig. 3.1(a-d). The outer ring corresponds to the contact line of the droplet that continues to move radially outward. The inner ring highlights the footprint of the bubble entrapped underneath the droplet interface [23-25]. For low undercooling $\Delta T \leq 8 \mathrm{~K}$, the inner ring disappears from the images for $t>0.0375 \mathrm{~ms}$, suggesting that the entrapped bubble detaches from the substrate and rises within the liquid. This detachment is directly related to the strong wetting of sapphire by hexadecane, as described in Ref. [26]. For higher undercooling $\Delta T>8 \mathrm{~K}$, on the contrary, the entrapped bubble freezes in contact with the substrate and at later times acts as a localised defect that causes delamination of the frozen splat.

For droplet impacts on surfaces maintained at low undercooling, the phase transition does not initiate instantly after a droplet touches the cold substrate. Instead, the crystal nucleation occurs randomly on the wetted area at timescales that are much 
larger than the typical timescale $t_{\mathrm{I}} \sim D / U$ during which the impact driven dynamics last. We believe, this is related to the high energy barrier for the formation of a stable crystal at temperatures close to the melting point [27, 28]. Therefore, at low $\Delta T$, facilitated by various inhomogeneities present in the system (surface roughness, thermal fluctuations, impurities in the liquid etc.) predominantly heterogeneous nucleation takes place. This also corroborates with the observed non-uniformity in the crystalseeding locations at small undercooling. For example, at $\Delta T=2 \mathrm{~K}$ (Fig. 3.1b), after the impact, the droplet spreads out in the form of a pancake of maximum diameter $D_{\mathrm{f}}$ until $t \sim 3 \mathrm{~ms}$, whereas the induction time of crystals is much larger, $t_{\mathrm{d}} \sim 40 \mathrm{~ms}$. With crystal induction time we refer to the time required to form a stable nucleus and its growth to the detectable size [29]. In this case, after the nucleation at a few sites $(N \sim 10)$, the crystallites grow in the form of needle-like structures (columnar dendrites), which eventually cover the whole wetted area of the substrate at $t \sim 2 \times 10^{3}$ $m s$. Note that the coverage of the wetted area by dendrites only indicates the end of the first phase of droplet freezing during which the liquid-substrate interface solidifies [12]. The bulk freezing of the liquid pancake takes place at much longer timescales $t_{\mathrm{f}} \sim \rho_{\mathrm{f}} L h^{2} / k_{\mathrm{f}} \Delta T \sim \mathscr{O}(10) \mathrm{s} ; \rho_{\mathrm{f}}$ and $k_{\mathrm{f}}$ are the density and the thermal conductivity of the solidified hexadecane, $L$ is latent heat released per unit mass, $h$ is the thickness of the frozen splat.

We noted that a slight increase in the substrate undercooling significantly influences the overall freezing morphology as well as the timescales associated with it. For $\Delta T=4.5 \mathrm{~K}$ (Fig. 3.1b), the induction time of crystals noticeably reduced to $t_{\mathrm{d}} \sim 5 \mathrm{~ms}>t_{\mathrm{I}}$ and nucleation occurred at a far larger number of locations $(N \sim \mathscr{O}(100))$ randomly distributed over the wetted area.

Furthermore, in stark contrast to the spatially erratic growth of columnar dendrites at $\Delta T=2 \mathrm{~K}$, here, the crystallites grew uniformly with nearly circular footprints in the plane parallel to the cold surface. However, a close inspection of the images reveals that the dendritic patterns persisted within these uniformly growing crystallites. This suggests that the crystal grains assume equiaxed dendritic morphology [28] at slightly increased $\Delta T$.

Strikingly, these dendritic morphologies were absent for droplets impacting on surfaces at $\Delta T \geq 8 \mathrm{~K}$. For instance, while a droplet spreads on a surface maintained at $\Delta T=8 \mathrm{~K}$ (Fig. 3.1c), the nucleation initiated at an enhanced rate in the middle of the evolving wetted area where the liquid temperatures are lowest (since the low thermal effusivity $e_{\mathrm{d}}=\sqrt{k \rho c_{\mathrm{p}}}$ of hexadecane limits the rate of heat removal from the warm droplet, the temperatures within the droplet are the lowest in the region that remains in contact with the cold substrate for the longest time). As a consequence, at $t_{\mathrm{d}} \sim 0.5$ $\mathrm{ms}<t_{\mathrm{I}}$, a cloud of micron-sized crystallites forms in the central region. Subsequently, this cloud grew nearly axi-symmetrically and covered the whole wetted area shortly after the droplet reaches its final resting size at $t \sim 3 \mathrm{~ms}$. Note that, at such high 


\subsection{Results and Discussion}

undercooling, the steep increase in the nucleation rate is linked to the corresponding decline in the energy barrier for the formation of a stable nucleus at higher $\Delta T$. This, in turn, reduces the dependence on the extrinsic factors for nucleation and induces prompt nucleation at an enhanced rate within the cooled liquid next to the substrate. In the following, we will see that the immediate nucleation at even higher $\Delta T$ leads to an unusual freezing morphology and dynamics, which, to the best of our knowledge, has not been reported earlier.

For $\Delta T=11 \mathrm{~K}$ (Fig. 3.1d), we observed that shortly after the impact of the drop, a cloud of crystallites appeared in the middle of the wetted area at $t_{\mathrm{d}} \sim 0.112$ $\mathrm{ms}$. The footprint of this cloud grew in size like in the previous case; however, at $t \sim 0.162 \mathrm{~ms}$, this cloud suddenly evolved into a circular band (frozen front) that moved radially outwards. Subsequently, while this frozen front moves towards the advancing contact line of the droplet, a second cloud emerges in the middle of the wetted area at $t \sim 0.275 \mathrm{~ms}$. This cloud as well evolves into a frozen front that moves out from the central region. Interestingly, this process repeated a number of times, resulting in the sequential advection of several frozen fronts before the dropletsubstrate interface completely solidified at $t \sim 3.625 \mathrm{~ms}$. It is important to point out that the sequential advection of the frozen fronts did not occur at a fixed interval of time $\delta t$. In time this interval increased monotonically. We measured that the initial two frozen fronts move out from the central region in the intervals of $\delta t \sim 0.1625$ $\mathrm{ms}$, whereas the subsequent frozen fronts appear at $\delta t>0.1625 \mathrm{~ms}$; see Fig. 3.2c. This striking increase in the time interval for the crystallite front emission is addressed in detail later in the discussion. Furthermore, we noted that the impact velocity had non-significant influence on $\delta t$. This was confirmed by varying the Weber number in between $\mathrm{We}=120-450$.

The physical mechanism responsible for this unusual freezing morphology is outlined schematically in Fig. 3.2a. We hypothesize that while a droplet spreads after the impact onto a highly undercooled surface, a thin layer of liquid next to the cold substrate rapidly cools down. Consequently, immediate nucleation occurs within this cooled liquid layer, as well as on the solid-liquid interface. The nucleation first initiates near the location of the droplet impact. Subsequently, the nucleated crystals, upon growing to a critical size $\left(\mathrm{r}_{c} \sim \delta_{v} / 2\right.$, the thickness of the viscous boundary layer) at $t=t_{\mathrm{c}} \ll t_{\mathrm{I}}$, are advected by the radial flow inside the droplet to form a frozen front. The whole process repeats each time the crystals nucleated in the middle of the wetted area are swept by the radial flow inside the droplet, leading to the sequential advection of the frozen fronts. Therefore, the growth rate of crystals within the viscous boundary layer next to the substrate dictates the overall freezing morphology by controlling the timescale at which the frozen fronts are advected. Since the growth of crystals in the vicinity of the undercooled surface strongly depends on the temperature of the substrate-liquid interface $\left(T_{\mathrm{sl}}\right)$, it arises as the most crucial parameter of our system. 
Qualitatively, the overall freezing morphology did not change for a further increase in $\Delta T$; see Fig. 3.2b. However, lowering of the substrate temperature significantly influenced the two important timescales associated with the freezing phenomenology, namely the induction time $t_{\mathrm{d}}$ and the periodicity $\delta t$. For experiments performed at $\Delta T=14.5 \mathrm{~K}$ and $\Delta T=17.5 \mathrm{~K}$, the induction time drops down to lower values of $t_{\mathrm{d}}=0.0625 \mathrm{~ms}$ and $0.0375 \mathrm{~ms}$, respectively. Similarly, the $\delta t$ for the initial frozen fronts in these cases reduced to $0.0875 \mathrm{~ms}$ and $0.050 \mathrm{~ms}$, respectively; see Fig. 3.2c. The prompt inception of the frozen fronts in these cases led to the accumulation of crystals near the advancing contact line at early stages of spreading after impact, $t<0.5 D / U \sim \mathscr{O}(0.5) \mathrm{ms}$. However, we noted that the contact line always preceded the advected frozen fronts and the subsequent spreading of the droplet remained unaffected. Accordingly, the nondimensional maximum spreading diameter of the liquid pancake after drop impact followed the scaling derived for isothermal impacts, $\xi_{\max }=D_{\mathrm{f}} / D \sim 0.78 \operatorname{Re}^{\frac{1}{5}}[30,31]$; see Fig. 3.2d. This indicated that the kinetic energy loss due to the solidification was too small to affect the extent of droplet spreading. This was further re-affirmed by the large values of the Prandtl number $\operatorname{Pr}=\mu / \rho \kappa_{1} \gg 1$ for liquid hexadecane, which signifies that the viscous effects dominate over the thermal effects during the impact of a droplet onto a cold surface; $\kappa_{1}$ is the thermal diffusivity of liquid hexadecane.

More quantitative insight into the sequential advection of frozen fronts can be gained from the theoretical analysis presented below. Firstly, using classical nucleation theory we estimate the growth rate of crystals $(\mathscr{V})$ in the vicinity of the substrate-liquid interface at temperature $T_{\mathrm{sl}}$. This yields:

$$
\mathscr{V}=\frac{\mathrm{d} r}{\mathrm{~d} t}=\frac{D}{\lambda}\left[1-\exp \left\{-\frac{M \Delta G_{f, v}}{\rho N_{\mathrm{A}} k_{\mathrm{B}} T_{\mathrm{sl}}}\right\}\right],
$$

where, $M=226.448 \mathrm{~g} / \mathrm{mol}$ is the molar mass of hexadecane, $N_{\mathrm{A}}$ is the Avogadro constant, $k_{B}$ is the Boltzmann coefficient, $\lambda$ is the mean free path of a liquid molecule, $D$ is the diffusion coefficient, $\Delta G_{f, v}=L\left(T_{\mathrm{m}}-T_{\mathrm{sl}}\right) / T_{\mathrm{m}}$ is the volumetric free energy difference between solid and liquid. The derivation of the growth rate of a crystal is provided in the section 3.C. Note that the temperature of the solid-liquid interface $T_{\mathrm{sl}}$ in Eq. (3.1) is unknown. Here, we adopted two separate strategies to estimate this temperature. These provide appropriate estimates of $T_{\mathrm{s} l}$ during the early and the later stages of droplet impact.

Since, at early times, only a small fraction of liquid in the middle of the wetted area transforms into crystallites, we ignore the latent heat released during the solidification. This allows us to approximate the interface temperature $T_{\mathrm{sl}}$ as the contact temperature between two semi-infinite bodies (droplet and substrate) that are bought into contact [32], as:

$$
T_{\mathrm{sl}}=T_{\mathrm{s}}+\left(T_{\mathrm{d}}-T_{\mathrm{s}}\right) \frac{1}{1+e_{\mathrm{s}} / e_{\mathrm{d}}},
$$




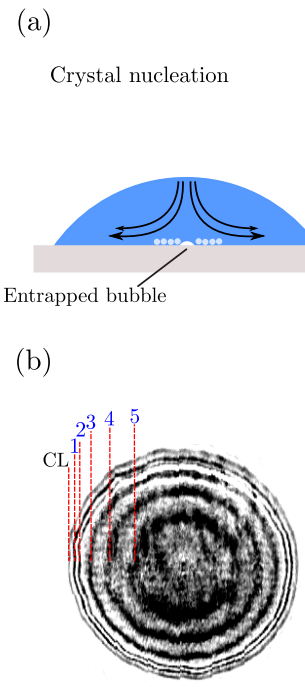

$\Delta T=17.5 \mathrm{~K}$

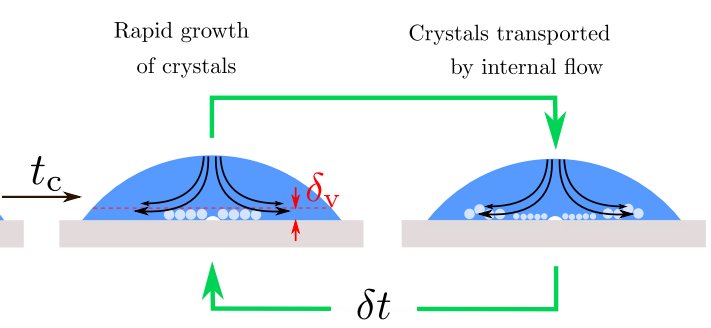

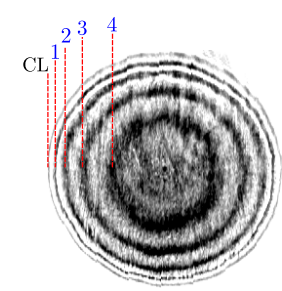

$\Delta T=14.5 \mathrm{~K}$

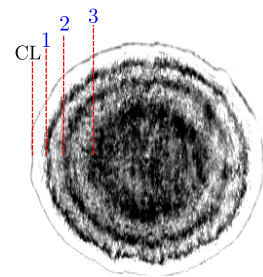

$\Delta T=11 \mathrm{~K}$

(c)

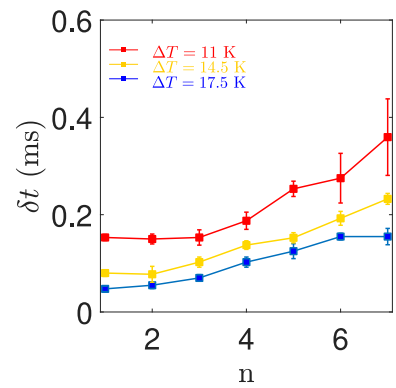

(d)

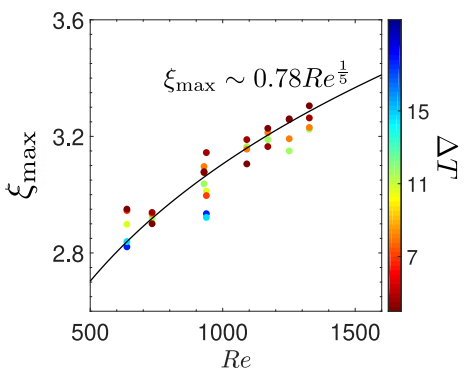

Figure 3.2: (a) Schematic representation of the physical mechanism responsible for the sequential advection of frozen fronts during the impact of a droplet on an undercooled surface with $\Delta T \geq 11 \mathrm{~K}$. (b) Three experimental snapshots recorded at $t=0.5 \mathrm{~ms}$ after the impacting droplet makes contact with the surfaces maintained at $\Delta T=17.5 \mathrm{~K}, 14.5 \mathrm{~K}$ and $11 \mathrm{~K}$. The higher the $\Delta T$, the quicker the advection of frozen fronts. At $\Delta T=17.5 \mathrm{~K}$ and $14.5 \mathrm{~K}$ the advected crystallites accumulate near the advancing contact line (CL) during early stages of the droplet spreading after impact, however the CL always precedes the advected frozen fronts. (c) Variation of time-interval $\delta t$ for successive frozen fronts (n). (d) Influence of freezing on the maximum spread of the droplet. In our system, the non-dimensional maximum spreading factor $\xi_{\mathrm{max}}=D_{\mathrm{f}} / D$ follows the scaling derived for iso-thermal impact, $\xi_{\max } \sim 0.78 \mathrm{Re}^{\frac{1}{5}}$ (solid line). 
$e=\sqrt{\kappa \rho c_{\mathrm{p}}}$ is the thermal effusivity, where the subscripts $s$ and $d$ denote the substrate and droplet, respectively. In contrast, at later times, due to the continuous nucleation and growth of crystallites a significant amount of latent heat is released near the substrate (Stefan number Ste $=c_{\mathrm{p}} \Delta T / L<1$ ). This results in the localized heating of the substrate. Therefore, to estimate $T_{\mathrm{sl}}$ at later times, we solve the two-phase Stefan problem with phase change using the Schwarz-solution [32,33]. The details of this model are provided in section 3.B.

According to Eq. 3.1, for a fixed value of $T_{\mathrm{sl}}$, a crystal grows linearly in time $\left(r_{\mathrm{c}} \propto t\right)$, whereas, the thickness of the viscous boundary layer inside an impacting droplet grows as $\delta_{\mathrm{v}} \sim 1.88 \sqrt{v t}$ [30]. Therefore, a crossover occurs when the crystal size becomes comparable to the thickness of the viscous boundary layer at

$$
t_{\mathrm{c}} \sim 0.88 \frac{v}{\mathscr{V}^{2}}
$$

Since we assume that the growing crystals are transported instantly after they reach the critical size $\left(r_{\mathrm{c}} \sim \delta_{\mathrm{v}} / 2\right)$, the crossover time $t_{\mathrm{c}}$ provides an estimate of the time interval between two successive frozen fronts. A comparison between the time interval $t_{\mathrm{c}}$ computed from our model at different $\Delta T$ and the corresponding experimental measurements of $\delta t$ is shown in Fig. 3.3. Despite the sensitivity of the model on various microscopic parameters that are not easily quantified, the model predictions for $\delta t$ are in reasonable agreement with the experimentally measured values. Our model successfully estimates the low values of $\delta t$ during the early times as well as the delay in the periodicity at later times. This also confirms that the rise in $\delta t$ at later times is related to the increase in $T_{\mathrm{sl}}$ due to the release of latent heat during solidification which slows down the growth rate of crystals. However, note that at later times, a complex interplay between the thickening of the viscous boundary later and slow growth rate of crystals determines $\delta t$, which is not included in the model. We must also point out that the deviation between the model predictions and the experimental measurements increases for larger $\Delta T$. We suspect this difference arises due to the non-equilibrium effects during the impact of a droplet. In our model we only include the slow diffusion-controlled growth of crystals in a quiescent undercooled liquid, which may not be an accurate description of the conditions during the nucleation at the early stages of the droplet impact. An accurate estimation would require a detailed modeling of the system which is beyond the scope of this work.

Finally, we shift our focus to the long term behaviour of the frozen splat. After the impact of a droplet on the undercooled substrate, it spreads out and slowly solidifies to form a thin crust of thickness $h \sim 150 \mu \mathrm{m}$ in time $t_{\mathrm{f}}$. At times much larger than $t_{\mathrm{f}}$, we noted that this thin solidified crust detaches from the substrate. This behaviour is similar to the delamination of the frozen metallic splats recently reported in Ref. [34]. It was reported that the delamination of tin splats initiates at the edges and creeps towards to the centre. Moreover, the extent increases for high $\Delta T$. The authors 


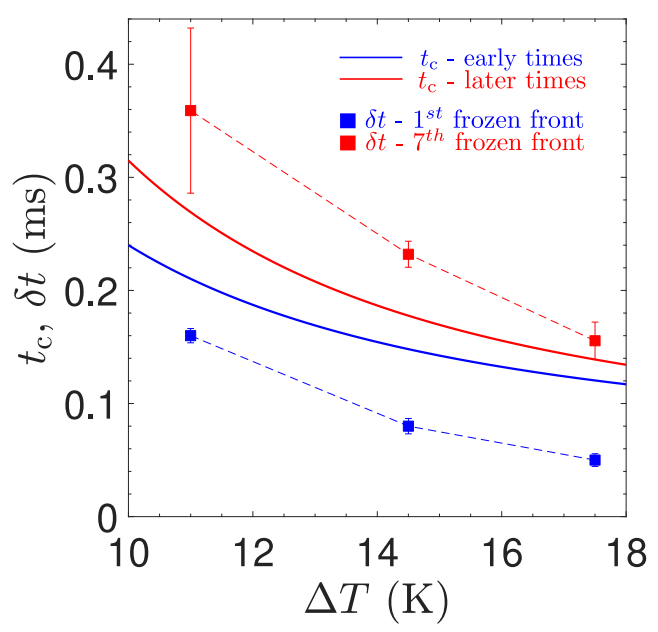

Figure 3.3: Comparison between the crossover time $t_{\mathrm{c}}$ determined from the model and the interval time $\delta t$ between successive frozen fronts measured in the experiments at different $\Delta T$. Blue (red) symbols indicate the experimental $\delta t$ for frozen fronts at early (late) stages of the droplet spreading. The blue (red) solid line highlights the prediction from the model using the substrate-liquid interface temperature estimated without (with) including latent heat released during solidification at early (late) stages of droplet spreading.

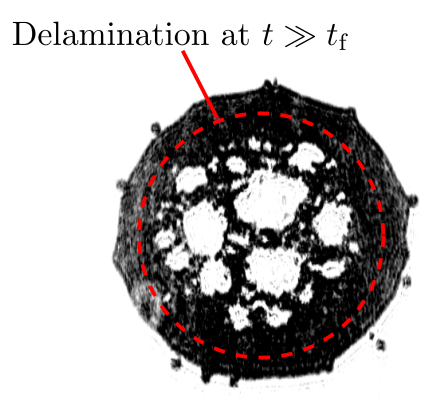

(a) $\Delta T=4.5 \mathrm{~K}$

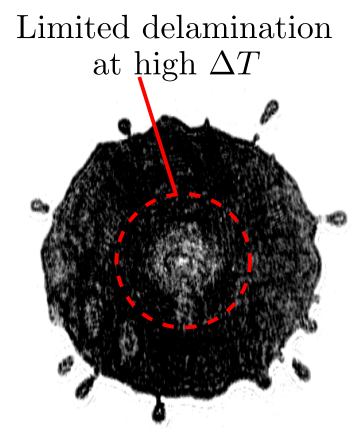

(b) $\Delta T=11 \mathrm{~K}$

Figure 3.4: Experimental snapshots showing the delamination behaviour of the frozen splat at $t \sim \mathscr{O}(10 s)>t_{\mathrm{f}}$. Contrary to the previous experimental reports for metallic droplet [34], in our case the delamination predominantly occurs in the central region of the frozen splat, and the extent of delamination reduces for higher $\Delta T$ 
argued that delamination is triggered by combination of thermo-mechanical stresses and interfacial defects at the bottom of the frozen splat. On the contrary, we noted that, at low $\Delta T$, the delamination of a hexadecane splat initiates at random locations (away from the boundaries) and eventually most of the central area of the frozen splat detaches from the substrate; see Fig. 3.4. Moreover, with increasing $\Delta T$, the extent of delamination reduces and remains limited to the central region of the frozen splat. Since no interfacial defects were observed for the hexadecane splats, we believe that in our system the delamination of the frozen splat could be driven by a peculiar crystallisation behaviour of hexadecane. It is known that $n$-even alkanes crystallise to a stable Triclinic crystal state through a so-called metastable Rotator phase [35, 36]. This metastable state is a weakly ordered crystal phase and at low supercooling, it can persist for long times [35]. Therefore, we believe that at low $\Delta T$, the build-up of compressive stresses due to thermal contraction is sufficient to cause interlayer movements of the rotator crystals which eventually leads to the delamination of the splat. In contrast, at high $\Delta T$, the transition between rotator and the highly ordered triclinic phase takes place rapidly, thus the extent of delamination remains limited near the location of droplet impact, where the entrapped bubble in the frozen splat acts as a localized defect.

\subsection{Conclusion}

In summary, by adapting the TIR technique to the freezing problem, we revealed a peculiar freezing morphology that originates from the complex interplay between the droplet-scale hydrodynamics and phase-transition effects at sufficiently high substrate undercooling. The kinetics of the advection of sequential frozen fronts observed at high $\Delta T$ is explained by combining the elements of classical nucleation theory and droplet-scale hydrodynamics. In addition, we reported a type of delamination behavior of frozen splats at times $t>t_{\mathrm{f}}$, which is drastically different from typical defecttriggered detachment processes that have been studied in detail [37]. In our system, the delamination of a frozen splat is driven by the existence of a weakly ordered transient crystalline phase which allows relative movement of the solidified material under thermomechanical stresses. Since TIR technique offers direct visualization of the nucleation events and crystal growth occurring next to the cold surface, it can be further be employed to study freezing in more complex situations, such as solidification of impacting droplet on liquid infused surfaces or patterned surfaces, which potentially can influence many industrial processes. 


\section{A. Materials and Methods}

\section{A Materials and Methods}

A schematic diagram of the experimental setup is shown in Fig. 3.5. In each experiment, a pendant drop was released from the tip of a needle with an outer(inner) diameter of 240(100) $\mu \mathrm{m}$. The balance between surface-tension and gravity forces ensured that all droplets were of a similar size $(1.58 \mathrm{~mm} \pm 20 \mu \mathrm{m})$. The employed liquid in the reported experiments was Hexadecane (99\%, Sigma-Aldrich), however, a few experiments were also performed with 1-Octadecene (Sigma-Aldrich) to confirm the non-exclusiveness of different freezing morphologies. Hexadecane is optically transparent at room temperature and has a melting point of $18^{\circ} \mathrm{C}$. The liquid has a density $\rho=770 \mathrm{~kg} / \mathrm{m}^{3}$, surface tension $\sigma=27 \mathrm{mN} / \mathrm{m}$, dynamic viscosity $\mu=3.47 \times 10^{-3} \mathrm{~Pa} \mathrm{~s}$, specific heat $c_{\mathrm{p}}=2310 \mathrm{~J} / \mathrm{kg} \mathrm{K}$, latent heat of fusion $L=2.3 \times 10^{5} \mathrm{~J} / \mathrm{kg}$ and thermal diffusivity $\alpha=8.40 \times 10^{-8} \mathrm{~m}^{2} / \mathrm{s}$. Here, we must emphasize that hexadecane is a nonvolatile liquid and has extremely low vapor pressure $P_{\text {vap }}=0.308 \mathrm{~Pa}$; thus, any evaporative precooling effects can be ignored in our experiments. The impact velocity $U$ was varied by adjusting the vertical distance between the horizontal substrate and the needle. The impact event was recorded in side-view as backlit shadow-graphs and in bottom-view via the total-internal-reflection technique. The impact velocity and shape/size of the droplet at the time of impact were measured from side-view images recorded with a high-speed camera (Photron APX-RS) at 10,000 to 14,000 fps with a macro lens. Bottom view observations (via TIR) were recorded using a high-speed camera (Photron Fastcam SA-X2) connected to a long-distance microscope (Navitar 12x Telecentric zoom system) at 80,000 fps.

The horizontal surface of a sapphire prism (Crystan Ltd.) of thermal conductivity $k_{\mathrm{s}}=34.60 \mathrm{Wm}^{-1} \mathrm{~K}^{-1}$ was used as a model surface in the experiments. It was placed in direct contact with a liquid-cooled aluminum holder. The average roughness of a similar prism was measured previously [22] to be less than $10 \mathrm{~nm}$. The temperature of the surface was measured before each experiment using a K-type thermocouple. Note that additional experiments were also performed under strongly reduced ambient pressure, ambient pressure $P_{a m b}=25 \mathrm{mbar}$, to understand the role of the air bubble entrapped during droplet impact and the formation of nanobubbles during the freezing of the droplet [38]. However, we did not measure any change in the overall kinetics of solidification due to reduced ambient pressure.

For TIR imaging, a $60 \mathrm{~mW}$ diode laser beam $(\lambda=643 \mathrm{~nm})$, expanded to $\approx 2$ $\mathrm{cm}$ diameter, was introduced to the prism via mirrors at a certain incident angle. The incident angle was carefully chosen such that it is greater than the critical angle $\theta_{\text {TIR }}>\sin ^{-1}\left(\frac{n_{\mathrm{d}}}{n_{\mathrm{s}}}\right)$, where $n_{\mathrm{d}}=1.43$ and $n_{\mathrm{s}}=1.76$ are the refractive indices of liquid hexadecane and sapphire, respectively. This ensured that in the bottom views recorded via TIR, the liquid in contact with the sapphire surface was not visible. However, an evanescent wave emerged in the droplet, whose intensity decayed in an exponential manner within one wavelength distance from the substrate. Hence, when a growing 


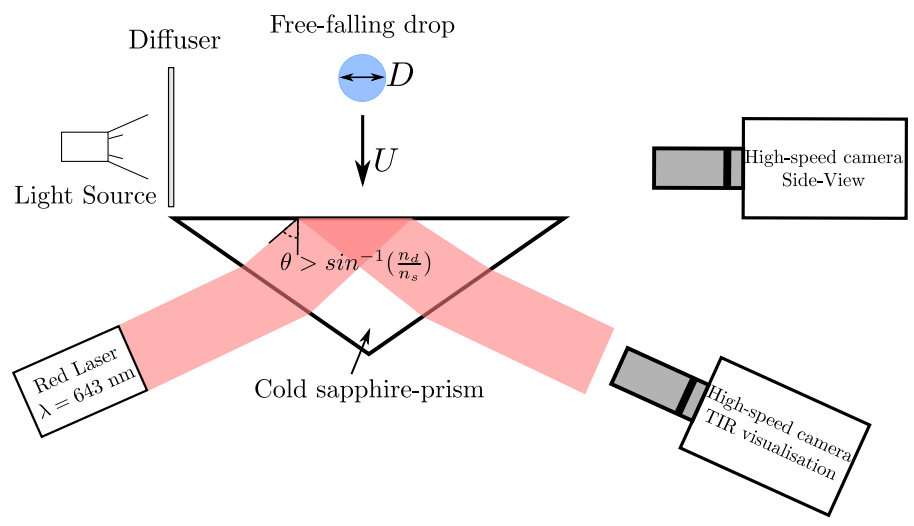

Figure 3.5: Schematic diagram of the experimental setup employing total-internal-reflection technique to visualise the freezing behaviour of an impacting droplet on undercooled surface.

crystal in the vicinity of the substrate interacted with the evanescent wave, it was visible in the images.

\section{B Two-phase Stefan problem}

As described in the main text, the rate of growth of crystals in the vicinity of the undercooled substrate crucially depends on the local temperature of the substrate $\left(T_{s l}\right)$ in contact with the warm liquid after the droplet impact. To estimate this unknown temperature, we formulate the transient heat transfer problem between the substrate and the impacting liquid as a 1-D two-phase Stefan problem involving phase change. We consider two semi-infinite bodies, the liquid hexadecane droplet and the undercooled sapphire substrate at temperatures $T_{\mathrm{d}}$ and $T_{\mathrm{s}}$, which are brought into contact at $t=0$ (see Figure 3.6). We assume that after a vertically falling droplet makes contact with the substrate, the heat transfer in both the liquid and the substrate takes place by pure conduction. Further, the solidification instantly starts at $t=0$ at the substrate-liquid interface and a sharp boundary, separating the liquid and the solidified material, moves normal to the substrate into the liquid at a velocity $v=\mathrm{d} z_{\text {int }} / \mathrm{d} t$. The temperature of this moving boundary remains constant $T=T_{\mathrm{m}}$. This allows us to solve the 1-D heat conduction problem in the normal direction to the undercooled substrate, including an evolving boundary condition for the phase change at the interface of liquid and solid hexadecane.

\section{B.1 The Schwarz solution}

An exact solution of the above described one-dimensional solidification problem is known as Schwarz solution [39]. It requires solution of the heat-conduction equation 


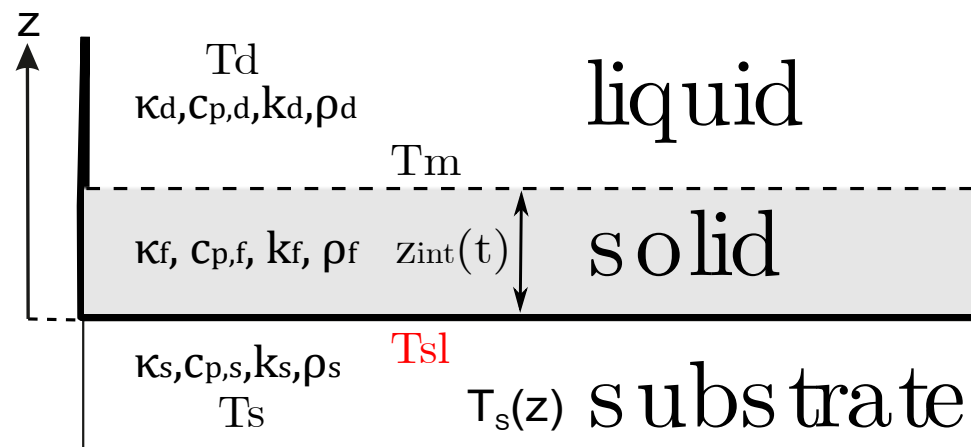

Figure 3.6: Solidification of liquid brought into contact with an undercooled substrate at temperature $T_{\mathrm{s}}<T_{\mathrm{m}}$. $T_{\mathrm{m}}$ is the melting temperature of hexadecane, $T_{s l}$ the temperature at the substrate-solid interface.

in three distinct regions:

$$
\text { in the substrate : } \quad \frac{1}{\kappa_{s}} \frac{\partial T}{\partial t}=\frac{\partial^{2} T}{\partial z^{2}}, \quad-\infty<z \leq 0
$$

within the solidified material : $\quad \frac{1}{\kappa_{f}} \frac{\partial T}{\partial t}=\frac{\partial^{2} T}{\partial z^{2}}, \quad 0<z<z_{\text {int }}$;

$$
\text { within the liquid : } \quad \frac{1}{\kappa_{d}} \frac{\partial T}{\partial t}=\frac{\partial^{2} T}{\partial z^{2}}, \quad z \leq z_{\text {int }}<\infty,
$$

with the following boundary conditions:

$$
\begin{gathered}
T(z \rightarrow-\infty, t)=T_{s}, \\
T(z \rightarrow \infty, t)=T_{d}, \\
T\left(z=z_{\text {int }}, t\right)=T_{m} .
\end{gathered}
$$

Note that $\kappa$ in eq. (1)-(3) is the thermal diffusivity of the respective materials.

\section{Moving interface}

The boundary condition at the moving interface $z_{\text {int }}(t)$ is derived via conservation of energy. We assume that the latent heat generated during the phase-change is dissipated through both the solidified material into the cold substrate as well as into the liquid. This yields:

$$
\left.k_{f} \frac{\partial T}{\partial z}\right|_{i n t}-\left.k_{d} \frac{\partial T}{\partial z}\right|_{i n t}=\rho L \frac{d z_{i n t}}{d t},
$$


where $k$ is the thermal conductivity of the respective media and $\rho$ and $L$ are the density and the latent heat of fusion of the liquid droplet.

A self-similar solution of the equations (3.4a) - (3.4c) with boundary conditions (4)-(6) can be written in terms of the similarity variable $\eta=z / 2(\kappa t)^{\frac{1}{2}}$ : This yields:

$$
\begin{array}{rr}
T=T_{s}+\sqrt{\frac{e_{f}}{e_{s}}} \frac{T_{m}-T_{s}}{\operatorname{erf} \eta+\sqrt{\frac{e_{f}}{e_{s}}}}\left(1+\operatorname{erf} \frac{z}{2\left(\kappa_{s} t\right)^{1 / 2}}\right), & -\infty<z \leq 0 ; \\
T=T_{s}+\sqrt{\frac{e_{f}}{e_{s}}} \frac{T_{m}-T_{s}}{\operatorname{erf} \eta+\sqrt{\frac{e_{f}}{e_{s}}}}+\frac{T_{m}-T_{s}}{\operatorname{erf} \eta+\sqrt{\frac{e_{f}}{e_{s}}}} \operatorname{erf} \frac{z}{2\left(\kappa_{f} t\right)^{1 / 2}}, & 0<z<z_{\text {int }} ; \\
T=T_{d}-\frac{T_{d}-T_{m}}{\operatorname{erfc}\left(\eta \kappa_{f} / \kappa_{d}\right)} \operatorname{erfc} \frac{z}{2\left(\kappa_{d} t\right)^{1 / 2}}, \quad z \leq z_{\text {int }}<\infty,
\end{array}
$$

Furthermore, the interface energy-balance equation (7) provides the additional relationship for the determination of parameter $\eta=z_{\text {int }} / 2\left(\kappa_{f} t\right)^{\frac{1}{2}}$ :

$$
\frac{\operatorname{Ste}_{s} \frac{e_{s}}{e_{f}} e^{-\eta^{2}}}{1+e_{s} / e_{f} \operatorname{erf} \eta}-\frac{\operatorname{Ste}_{d} \frac{e_{d}}{e_{f}} e^{-\eta^{2} \kappa_{f} / \kappa_{d}}}{\operatorname{erfc}\left(\eta \kappa_{f} / \kappa_{d}\right)}=\eta \sqrt{\pi}
$$

In (3.9)-(3.12) $e=\sqrt{k \rho c_{p}}$ is the thermal effusivity, Ste $_{s}=c_{p, f}\left(T_{m}-T_{s}\right) / L$ and Ste $_{d}=c_{p, f}\left(T_{d}-T_{m}\right) / L$ are the Stefan numbers of the substrate and the droplet, respectively; $c_{p, f}$ is the heat capacity of the solidified liquid.

Finally, the contact temperature $T_{s l}$ is calculated by setting $z=0$ into equation (9), which leads to:

$$
T_{s l}=T_{s}+\sqrt{\frac{e_{f}}{e_{s}}} \frac{T_{m}-T_{s}}{\operatorname{erf} \eta+\sqrt{\frac{e_{f}}{e_{s}}}} .
$$

As can be seen from equation 3.13, the contact temperature is time-independent. It stays constant for given initial conditions, however the temperatures within the substrate, the solidified material and the liquid vary in time; see Figure 3.7. Further, in Fig. 3.8 we plot the variation of $T_{s l}$ with $T_{S}$ computed from the 1-D model described above.

\section{C Kinetic model for cluster growth: Interface controlled growth}

In the framework of the classical nucleation theory, it is proposed that clusters of atoms in the configuration of the transformation product arise within the original material 
3.C. Kinetic model for cluster growth: Interface controlled growth
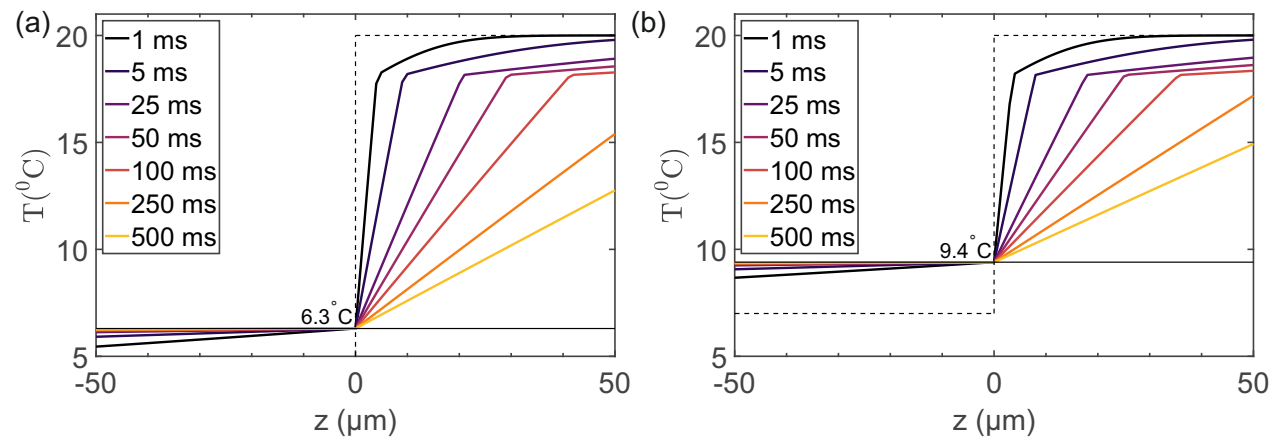

Figure 3.7: Model solution to the Stefan problem for solidifying hexadecane brought into contact with sapphire. Negative $\mathrm{z}$ corresponds to the sapphire, positive $\mathrm{z}$ to the solidifying hexadecane. The dashed lines indicate the initial temperature profile $(t=0)$, the solid line the contact temperature $T_{s l}$ (a) $T_{d}=20^{\circ} \mathrm{C}$ and $T_{s}=3.5^{\circ} \mathrm{C}$. The contact temperature is $T_{s l}=6.3^{\circ} \mathrm{C}$. (b) $T_{d}=20^{\circ} \mathrm{C}$ and $T_{s}=7^{\circ} \mathrm{C}$. The contact temperature is $T_{s l}=9.4^{\circ} \mathrm{C}$

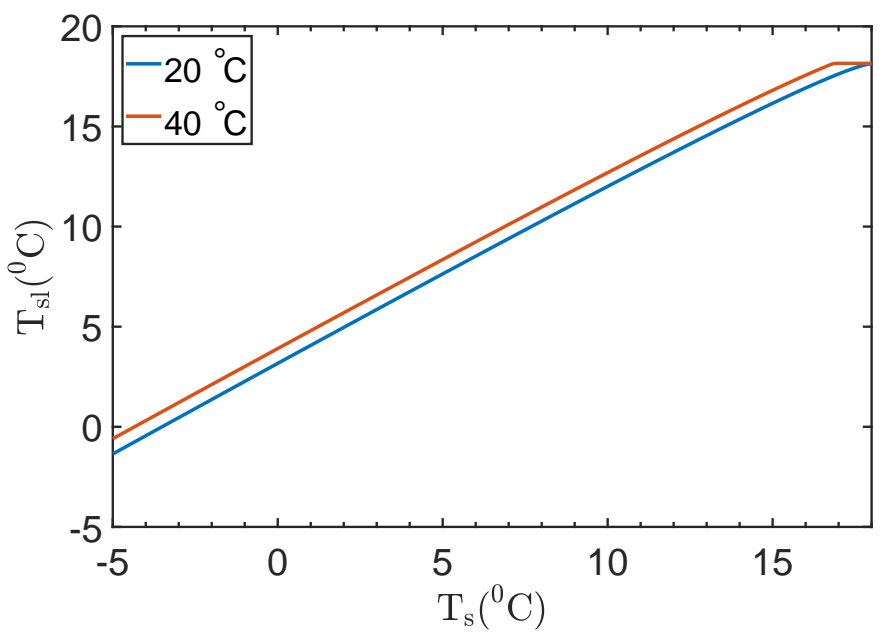

Figure 3.8: Variation of contact temperature $T_{s l}$ with substrate undercooling, for two droplet temperatures: $T_{d}=20^{\circ} \mathrm{C}$ (blue) and $T_{d}=40^{\circ} \mathrm{C}$ (red). 

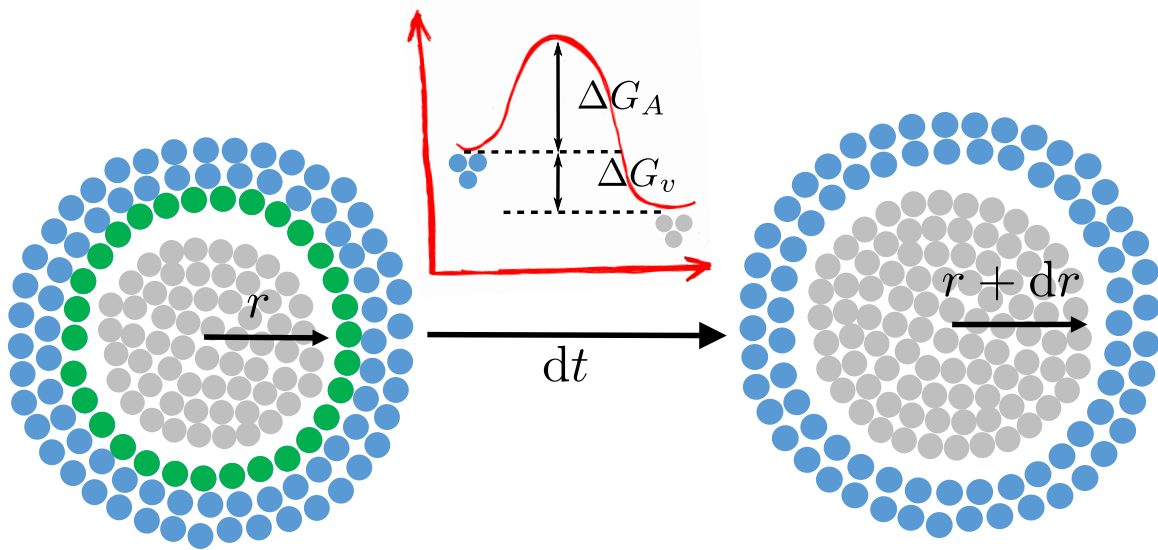

Figure 3.9: Schematic diagram showing the growth of a nuclei of radii $r$ in time $\mathrm{d} t$.

[40, 41]. Initially, the clusters are small and unstable because of a high surface-tovolume ratio. Eventually, some of the clusters grow to a critical size beyond which they are stable. The formation of such small and stable clusters of transformation product is called nucleation. Once formed, these clusters continue to grow in time, while the excess free energy of formation is positive. The growth of clusters can either be diffusion or interface controlled. In this work we assume interface controlled growth of hexadecane nuclei. Figure 3.9 shows a schematic of the growth of a cluster, surrounded by metastable medium, in time $\mathrm{d} t$. The corresponding change in the free energy during the growth of a nucleus is also highlighted in Figure 3.9; $\Delta G_{A}$ is the interfacial activation energy and $\Delta G_{v}$ is the latent heat released per unit volume during the growth. In such a scenario we follow the analysis presented by Turnbull and Fisher [40] to determine the growth rate of a cluster during phase change. Note that in this analysis we assume homogeneous nucleation.

In this formalism, we compute the net forward rate of phase transformation (liquid to solid) :

$$
\frac{\mathrm{d} n}{\mathrm{~d} t}=n_{s} v\left[\exp \left\{-\frac{\Delta G_{A}}{k_{B} T}\right\}-\exp \left\{-\frac{\Delta G_{A}+|v| \Delta G_{v}}{k_{B} T}\right\}\right],
$$

here, $n_{s}$ are the number of atoms surrounding a cluster per unit surface area of the nucleus, $v$ is the lattice vibration frequency, $|v|=M / \rho N_{A}$ is the volume of a single atom, $M$ is the molar mass of the liquid, $\rho$ is the density, $N_{A}$ is the Avogadro constant and $k_{B}$ is the Boltzmann constant; $T$ is the temperature of the melt surrounding the nucleus. Since in the experiments the crystals grow predominantly close to the liquidsubstrate interface, we take $T=T_{\mathrm{sl}}$. Also note that the net forward rate $\mathrm{d} n / \mathrm{d} t$ has units of $\mathrm{m}^{-2} \mathrm{~s}^{-1}$. To estimate the growth rate $\mathscr{V}$ with dimensional units of $\mathrm{ms}^{-1}$, we 
multiply the above equation with the volume of a single atom, this leads to

$$
\mathscr{V}=\frac{\mathrm{d} r}{\mathrm{~d} t}=\lambda v \exp \left\{-\frac{\Delta G_{A}}{k_{B} T}\right\}\left[1-\exp \left\{-\frac{|v| \Delta G_{v}}{k_{B} T_{s l}}\right\}\right],
$$

where, $\lambda=n_{s} \cdot|v|$ is the single atomic jump also referred to as the mean free path. The above expression can be further simplified using the definition of the diffusion coefficient $D=\lambda^{2} v \exp \left\{-\frac{\Delta G_{A}}{k_{B} T}\right\}$ to

$$
\mathscr{V}=\frac{D}{\lambda}\left[1-\exp \left\{-\frac{|v| \Delta G_{v}}{k_{B} T_{s l}}\right\}\right],
$$

The above treatment of the cluster growth excludes many effects which may arise during the solidification of a droplet during the impact, such as mobility of the metastable medium and the clusters, configurational frustrations between clusters, non-stoichiometric clusters, etc. For further discussion we refer to Ref. [42].

\section{D Visualisation}

The TIR method technique employed in this work has the advantage to be only sensitive to measure processes in a thickness smaller than the first micrometer of the droplet. The experiments were performed on the horizontal surface of a sapphire prism placed in direct contact with a liquid-cooled aluminum holder. We used a $643 \mathrm{~nm}$ diode laser, expanded through a set of lenses to illuminate the complete footprint of an impacting droplet. The incident angle of the laser $\theta$ was chosen to be larger than the critical angle $\theta_{c}=\sin ^{-1}\left(n_{2} / n_{1}\right)$ for the TIR imaging to work. Here $n_{2}$ is the refractive index of the sapphire prism and $n_{1}$ that of the medium across the interface. As a consequence, total internal reflection occurs where the sapphire is wetted by liquid hexadecane, whereas the localised scattering/diffraction of the incident laser beam at the contact line of the spreading droplet and growing crystals at the substrate-liquid interface enabled us to make a direct visulaisation of the solidification and spreading kinetics. Note that the image recorded from the TIR setup were distorted (elliptical rather than circular). The back transformation of these images was achieved by the method described in Ref. [21]. In this article, only reconstructed images are shown.

Further, to confirm that the localised diffraction of the incident laser is the main reason for visibility of contact line and solidified material in the TIR setup, we performed a simulation based on the time-independent Helmholtz-equation in the three media, using the code developed by Osnabrugge et. al. [43]. As shown in Figure 3.10, we numerically solve for a Gaussian beam that is propagating from left to right and is reflected on the prism surface. The domain is normalized by the incident wave 


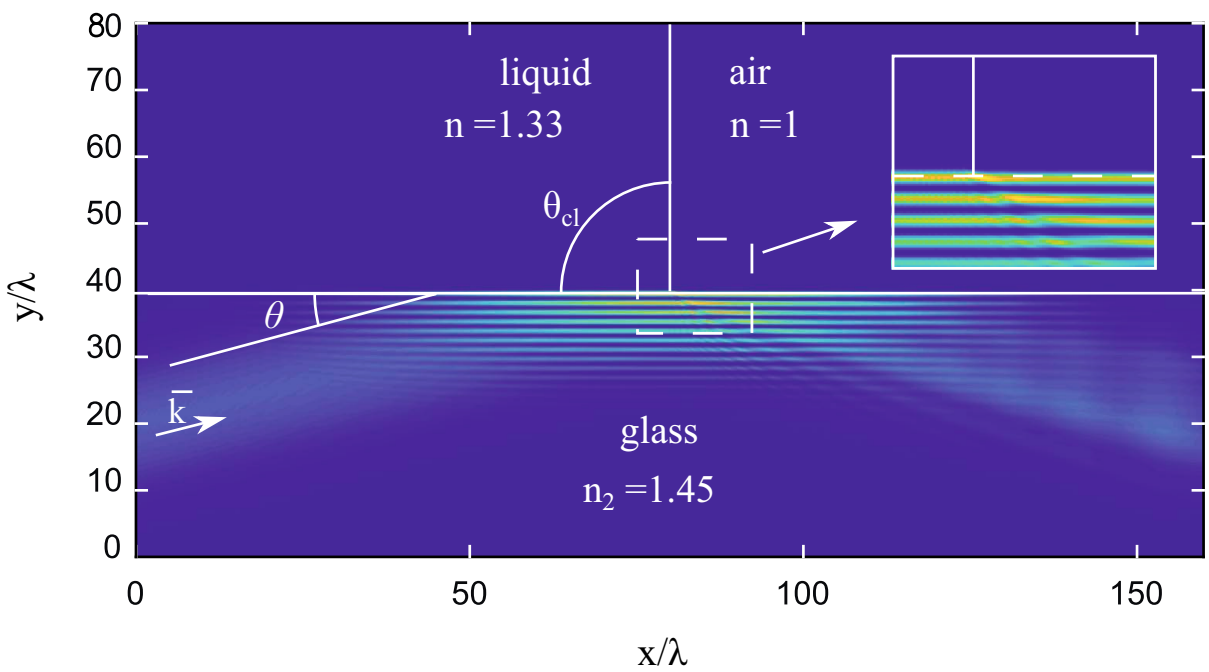

Figure 3.10: Simulation results, showing the normalized intensity field of a collimated laser beam of width $10 \lambda$. The wave vector $\vec{k}$, which determines the incident angle $\theta$ with the glass surface, is chosen such that the light undergoes total internal reflection for both the liquid (left) and air (right) phases. The vertical white line represents the liquid-air interface, whereas the inset reveals the details at the contact line, showing the diffraction patterns leading to the visibility of the contact line.

length $\lambda$ and the beam width is $10 \lambda$. The prism material has a refractive index of $n=1.45$; and $n=1$ is used on the right, for the air, whereas $n=1.33$ is used for the liquid part. The resulting normalized electric field is represented by the color between zero (blue) and 1 (yellow). The inset magnifies the details at the contact line. The difference in decay length $d=\lambda_{0} /\left(4 \pi n_{1}\right)\left(\sin ^{2} \theta-\left(n_{2} / n_{1}\right)^{2}\right)^{-1 / 2}$ for the glass-air and glass-liquid interfaces results in the intensity fluctuations in the right part of the glass domain. However, the finite aperture of optical systems used in experiments cannot resolve this small scale feature. The diffraction pattern captured is used to identify the contact line position. 


\section{References}

[1] J. Madejski, "Solidification of droplets on a cold surface", Int J. Heat Mass Transf. 19, 1009-1013 (1976).

[2] M. Pasandideh-Fard, Y. M. Qiao, S. Chandra, and J. Mostaghimi, "Capillary effects during droplet impact on a solid surface", Phys. Fluids 8, 650-659 (1996).

[3] M. Schremb, I. V. Roisman, and C. Tropea, "Normal impact of supercooled water drops onto a smooth ice surface: experiments and modelling", Journal of Fluid Mechanics 835, 1087-1107 (2018).

[4] T. Bennett and D. Poulikakos, "Splat-quench solidification: estimating the maximum spreading of a droplet impacting a solid surface", J. Mater. Sci. 28, 963-970 (1993).

[5] A. Alizadeh, M. Yamada, R. Li, W. Shang, S. Otta, S. Zhong, L. Ge, A. Dhinojwala, K. R. Conway, V. Bahadur, et al., "Dynamics of ice nucleation on water repellent surfaces”, Langmuir 28, 3180-3186 (2012).

[6] Z. Jin, H. Zhang, and Z. Yang, "Experimental investigation of the impact and freezing processes of a water droplet on an ice surface", International Journal of Heat and Mass Transfer 109, 716-724 (2017).

[7] R. Dhiman and S. Chandra, "Freezing-induced splashing during impact of molten metal droplets with high weber numbers", International journal of heat and mass transfer 48, 5625-5638 (2005).

[8] L. Mishchenko, B. Hatton, V. Bahadur, J. A. Taylor, T. Krupenkin, and J. Aizenberg, "Design of ice-free nanostructured surfaces based on repulsion of impacting water droplets", ACS Nano 4, 7699-7707 (2010).

[9] M. V. Gielen, R. de Ruiter, R. B. Koldeweij, D. Lohse, J. H. Snoeijer, and H. Gelderblom, "Solidification of liquid metal drops during impact", Journal of Fluid Mechanics 883 (2020).

[10] M. Schremb, I. V. Roisman, and C. Tropea, "Transient effects in ice nucleation of a water drop impacting onto a cold substrate”, Phys. Rev. E 95, 022805 (2017).

[11] L. Wang, W. Kong, F. Wang, and H. Liu, "Effect of nucleation time on freezing morphology and type of a water droplet impacting onto cold substrate", Int J. Heat Mass Transf. 130, 831-842 (2019).

[12] M. Schremb, J. M. Campbell, H. K. Christenson, and C. Tropea, "Ice layer spreading along a solid substrate during solidification of supercooled water: Experiments and modeling”, Langmuir 33, 4870-4877 (2017). 
[13] F. Gao and A. A. Sonin, "Precise deposition of molten microdrops: the physics of digital microfabrication", Proc. R. Soc. London, Ser. A 444, 533-554 (1994).

[14] C. W. Visser, R. Pohl, C. Sun, G.-W. Römer, B. Huis in 't Veld, and D. Lohse, " $3 \mathrm{~d}$ printing: Toward $3 \mathrm{~d}$ printing of pure metals by laser-induced forward transfer (adv. mater. 27/2015)", Adv. Mater. 27, 4103-4103 (2015).

[15] A. Lawley and D. Apelian, "Spray forming of metal matrix composites", Powder Metall. 37, 123-128 (1994).

[16] R. Evans, A. Leatham, and R. Brooks, "The osprey preform process", Powder Metall. 28, 13-20 (1985).

[17] Q. Liu and M. Orme, "High precision solder droplet printing technology and the state-of-the-art", J. Mater. Process. Technol. 115, 271-283 (2001).

[18] H. Mizoguchi, T. Abe, Y. Watanabe, T. Ishihara, T. Ohta, T. Hori, T. Yanagida, H. Nagano, T. Yabu, S. Nagai, et al., "100w 1st generation laser-produced plasma light source system for hvm euv lithography", in Extreme Ultraviolet (EUV) Lithography II, volume 7969, 796908 (International Society for Optics and Photonics) (2011).

[19] V. Y. Banine, K. Koshelev, and G. Swinkels, "Physical processes in euv sources for microlithography", Journal of Physics D: Applied Physics 44, 253001 (2011).

[20] J. M. Kolinski, S. M. Rubinstein, S. Mandre, M. P. Brenner, D. A. Weitz, and L. Mahadevan, "Skating on a film of air: drops impacting on a surface", Phys. Rev. Lett. 108, 074503 (2012).

[21] M. Shirota, M. A. van Limbeek, D. Lohse, and C. Sun, "Measuring thin films using quantitative frustrated total internal reflection (ftir)", Eur. Phys. J. E 40, 54 (2017).

[22] M. Shirota, M. A. van Limbeek, C. Sun, A. Prosperetti, and D. Lohse, "Dynamic leidenfrost effect: relevant time and length scales", Phys. Rev. Lett. 116, 064501 (2016).

[23] S. Chandra and C. Avedisian, "On the collision of a droplet with a solid surface", Proc. R. Soc. London, Ser. A 432, 13-41 (1991).

[24] S. Thoroddsen, T. Etoh, K. Takehara, N. Ootsuka, and Y. Hatsuki, "The air bubble entrapped under a drop impacting on a solid surface”, J. Fluid Mech. 545, 203-212 (2005). 
References

[25] C. Josserand and S. T. Thoroddsen, "Drop impact on a solid surface", Ann. Rev. Fluid Mech. 48, 365-391 (2016).

[26] J. San Lee, B. M. Weon, J. H. Je, and K. Fezzaa, "How does an air film evolve into a bubble during drop impact?", Phys. Rev. Lett. 109, 204501 (2012).

[27] D. Turnbull, "Kinetics of solidification of supercooled liquid mercury droplets", J. Chem. Phys 20, 411-424 (1952).

[28] W. Kurz and D. J. Fisher, Fundamentals of Solidification, volume 1 (trans tech publications Aedermannsdorf, Switzerland) (1986).

[29] O. Söhnel and J. W. Mullin, "Interpretation of crystallization induction periods", J. Colloid Interf. Sci. 123, 43-50 (1988).

[30] I. V. Roisman, "Inertia dominated drop collisions. ii. an analytical solution of the navier-stokes equations for a spreading viscous film", Phys. Fluids 21, 052104 (2009).

[31] S. Wildeman, C. W. Visser, C. Sun, and D. Lohse, "On the spreading of impacting drops", J. Fluid Mech. 805, 636-655 (2016).

[32] H. S. Carslaw and J. C. Jaeger, "Conduction of heat in solids", Oxford: Clarendon Press, 1959, 2nd ed. (1959).

[33] T. Loulou and D. Delaunay, "The interface temperature of two suddenly contacting bodies, one of them undergoing phase change", Int J. Heat Mass Transf. 40, 1713-1716 (1997).

[34] J. de Ruiter, D. Soto, and K. K. Varanasi, "Self-peeling of impacting droplets", Nat. Phys. 14, 35 (2018).

[35] E. Sirota and A. Herhold, "Transient phase-induced nucleation", Science 283, 529-532 (1999).

[36] E. Sirota, "Supercooling, nucleation, rotator phases, and surface crystallization of n-alkane melts", Langmuir 14, 3133-3136 (1998).

[37] H.-h. Yu and J. W. Hutchinson, "Delamination of thin film strips", Thin Solid Films 423, 54-63 (2003).

[38] F. Chu, X. Zhang, S. Li, H. Jin, J. Zhang, X. Wu, and D. Wen, "Bubble formation in freezing droplets", Physical Review Fluids 4, 071601 (2019).

[39] H. S. Carslaw and J. C. Jaeger, Conduction of heat in solids, volume 2 (Oxford Science Publications, Oxford) (2016). 
[40] D. Turnbull and J. C. Fisher, "Rate of nucleation in condensed systems", J. Chem. Phys. 17, 71-73 (1949).

[41] K. F. Kelton, A. L. Greer, and C. V. Thompson, "Transient nucleation in condensed systems”, J. Chem. Phys. 79, 6261-6276 (1983).

[42] E. Clouet, "Modeling of Nucleation Processes", in ASM Handbook Vol. 22A, Fundamentals of Modeling for Metals Processing, edited by F. D.U. and S. S.L., 203-219 (Asm International) (2009).

[43] G. Osnabrugge, S. Leedumrongwatthanakun, and I. M. Vellekoop, "A convergent Born series for solving the inhomogeneous Helmholtz equation in arbitrarily large media”, J. Comput. Phys. 322, 113-124 (2016). 




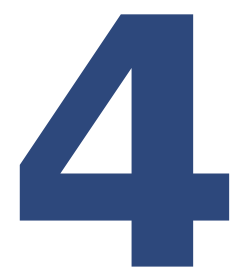

\section{Droplet impact on a supercooled surface and its subsequent solidification *}

When a drop impacts on a surface below its freezing temperature, it simultaneously spreads and solidifies. In the present work, tin droplet impact on isothermal surfaces below the freezing temperature is studied numerically. The geometrical Volumeof-Fluid interface method, with a height-function curvature is used to solve the hydrodynamical equations, extended with an enthalpy-porosity formulation to study solidification. The numerical method is validated against experiments available in the literature and favourably compared to earlier numerical methods. A study of $1 \mathrm{~mm}$ sized tin droplets is performed, showing various impact regimes: deposition, capillary ejection, and (partial) break-up. At early times, the spreading is found as for isothermal droplets, but later the solidification arrests the moving interface. The dependence of the spreading on the Weber (We) and the Stefan (Ste) number is quantified. We develop an analytical model for the maximum spreading diameter as function of dimensionless parameters, based on a combination of the hydrodynamics and the solidification rate. This model gives good estimates for the maximum droplet diameter of solidifying droplets.

*Submitted as: "Droplet impact on a supercooled surface and its subsequent solidification", R.B.J. Koldeweij and D. Lohse 


\subsection{Introduction}

Impact of droplets on a surface maintained below the freezing temperature of the liquid is a key phenomenon in many technologies. For instance, the solidification of liquid droplets is used in thermal spray coating, where a uniform coating is applied to a surface [1,2]. Further applications are flip-chip packaging [3-5] and three-dimensional printing of well defined structures $[2,6,7]$. More recently micro fabrication techniques to create well defined metal structures have been developed $[8,9]$. Also here drop solidification is crucial and challenging.

The solidification of droplets during impact depends on the interplay between the drop spreading dynamics and the simultaneous phase transition towards a solid particle. Isothermal droplet impact on solid surfaces, i.e. without phase transition of the droplet, has been studied extensively both numerically and experimentally [10-14]. The numerical solution of the interfacial dynamics of problems of this type has seen major progress in the last two decades [15], one of the main challenges being the balancing of the equilibrium condition between pressure and surface tension [16].

In parallel, the modelling of solidification of droplets has been investigated. In early work simple theoretical models were developed, assuming a velocity profile representing the flattening of a droplet [17] and simultaneously solving the Stefan problem for the phase transition from liquid to solid. Various improvements have been made to better describe the flow dynamics and solidification characteristics [18-20]. Later, numerical studies based on the solution of the Stefan problem were proposed by Ref. [21, 22], among others.

However, to accurately model the solidification of an impacting droplet, the combined effects of fluid flow and heat transfer with solidification needs to be calculated. A volume of fluid $(V o F)$ method with solidification was first proposed by Ref. [23]. In numerical methods based on this approach generally three strategies can be distinguished. Firstly, damping the momentum in the Navier-Stokes equation directly by imposing a penalty on the velocity of a (partly) solidified zone [23-27]. Secondly, increasing the viscosity of the partly solidified material [28-30]. Thirdly, by damping the momentum equation using a porous zone approximation, [31-34]. The available models make no use of adaptive re-meshing; therefore a combination of high resolution and efficient solving is not available. Furthermore, the various approaches available in the literature show a variation in accuracy, depending on the choice of a fitting parameter, the so-called contact resistance $[23,30]$.

To be able to accurately study droplet impact, in this chapter a numerical method is proposed in the $V o F$ class of fluid modelling, making use of adaptive remeshing. For the first time, the usage of height functions to accurately obtain the interface is combined with solidification of droplets. The multi-phase flow field is coupled to an enthalpy based equation for heat transfer. The porous zone approximation method [31] is used to dampen the momentum equation in case of solidification. 

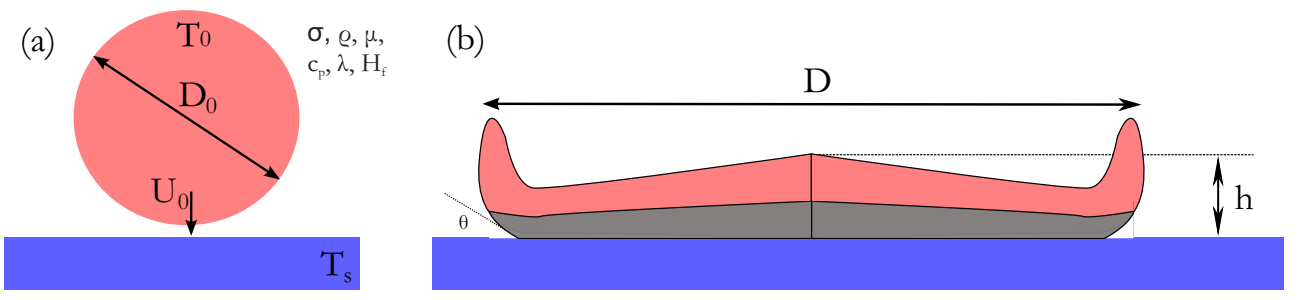

Figure 4.1: Sketch of an impacting and solidifying droplet. (a) A droplet with size $D_{0}$, velocity $U_{0}$ and temperature $T_{0}$ impacts on a surface with temperature $T_{s}$. What will be the spreading diameter $D$ and centre height $h$ as a function of liquid properties (surface tension $\sigma$, viscosity $\mu$, density $\rho$, heat capacity $c_{p}$, conductivity $\lambda$ and latent heat $H_{f}$ ) and surface contact angle $\theta$ (b) The hot liquid droplet (red), spreads and simultaneously freezes on a cold surface (blue) into a solid (grey). The colors are an artist impression and have no physical significance.

Most numerical studies focus on the description of a few impact scenarios, or describe the effect of the contact resistance [35-37]. Furthermore, various phenomena like thermal shrinkage [32], or non-equilibrium solidification [34] have been studied. Here we are interested in the behaviour of single metal droplet impact. Therefore we focus on a systematic approach of droplet spreading behaviour with solidification for millimetre sized tin droplets. Figure 4.1 sketches the impact of such a spherical droplet on a non-deformable, isothermal, supercooled surface. The question is to find the spreading extent $D$ of the droplet of initial size $D_{0}$, given its initial impact velocity $U_{0}$, its initial temperature $T_{0}$, its hydrodynamical (surface tension $\sigma$, viscosity $\mu$, density $\rho$ ) and thermal properties (heat capacity $c_{p}$, conductivity $\lambda$, latent heat $H_{f}$, freezing temperature $T_{f}$ ), and the surface properties; namely the liquid-solid (advancing) contact angle $\theta$ and 'the surface temperature $T_{s}<T_{f}$. This problem can be reduced to finding the spreading ratio $d / D_{0}$ as function of dimensionless parameters $D / D_{0}=f($ We, Re, $\theta$, Pe, Ste $)$, with the Weber number We, the Reynolds number Re, the Peclet number Pe and the Stefan number Ste defined as

$$
\text { We } \equiv \frac{\rho D_{0} U_{0}^{2}}{\sigma}, \operatorname{Re} \equiv \frac{\rho D_{0} U_{0}}{\mu}, \operatorname{Pe} \equiv \frac{D_{0} U_{0}}{k}, \text { Ste } \equiv \frac{c_{p} \Delta T}{H_{f}},
$$

respectively. Here $k=\lambda / \rho c_{p}$ is the thermal diffusivity of the liquid (which is often denoted as $\kappa$, however here curvature is denoted by $\kappa$ ) and $\Delta T=T_{f}-T_{s}$. These dimensionless numbers represent, respectively, the importance of surface energy to impact energy of the droplet, the ratio of inertia to viscosity of the droplet, the ratio of heat transfer due to convection compared to diffusion in the droplet, and the ratio of sensible to latent heat in the droplet.

We explain the model in detail in section 4.2 , followed by validation and a parameter sensitivity study in section 4.3 . In section 4.4 , the model is used to present 
a regime diagram and quantify droplet spreading behaviour for a $1 \mathrm{~mm}$ tin droplet impact, based on variations of temperatures from $25^{\circ} \mathrm{C}$ to $240^{\circ} \mathrm{C}(0<$ Ste $<0.75$ and impact velocities ranging from $0.1 \mathrm{~m} \mathrm{~s}^{-1}$ to $2 \mathrm{~m} \mathrm{~s}^{-1}(0.1<\mathrm{We}<55)$. Finally we present an approximate model based on energy conservation to compute the maximum spreading diameter of impacting droplets that simultaneously solidify, extending the earlier purely hydrodynamical model of Ref. [9] by thermal effects.

\subsection{Numerical model}

\subsubsection{Fluid model}

Describing the spreading behaviour and small scale heat transfer of an impacting droplet requires detailed calculation of the flow field. Therefore we use the open source solver GERRIS to simulate the impact of droplets on surfaces. GERRIS solves the Navier-Stokes equations with the Volume of Fluid ( $\mathrm{VoF}$ ) method using adaptive grid refinement[16, 38].

A gas-droplet system with densities $\rho_{g}$ and $\rho_{l}$ and viscosities $\mu_{g}$ and $\mu_{l}$ is simulated using the incompressible Navier-Stokes equations,

$$
\begin{gathered}
\nabla \cdot \vec{u}=0 ; \\
\frac{\partial \vec{u}}{\partial t}+(\vec{u} \cdot \nabla) \vec{u}=\frac{1}{\rho}\left(-\nabla p+\sigma \kappa \delta_{s} \vec{n}+\nabla \cdot\left(\mu\left(\nabla \vec{u}+(\nabla \vec{u})^{T}\right)\right)+S_{j}\right),
\end{gathered}
$$

where $\vec{u}$ is the fluid velocity and $p$ is the fluid pressure. The Dirac delta function $\delta_{s}$ ensures that the surface tension term is concentrated at the interface between the droplet and the air, with $\kappa$ and $\vec{n}$ being the curvature of and normal direction to the interface, respectively. The additional dissipation term $S_{j}$ is added to account for the momentum loss due to solidification and will be described later.

A schematic overview of the domain and adaptive grid is shown in Figure 4.2. The interface between the droplet and surrounding gas is tracked using the Volume of Fluid method on a Eulerian mesh. This method follows the phase fraction by the advection equation

$$
\frac{\partial G}{\partial t}+(\vec{u} \cdot \nabla) G=0
$$

Here $G$ is the phase fraction for a particular phase. It is defined as:

$$
G(\vec{x}, t)= \begin{cases}1 & \text { in the droplet; } \\ 0<G<1 & \text { at the interface between droplet and gas; } \\ 0 & \text { in the gas. }\end{cases}
$$




\subsection{Numerical model}

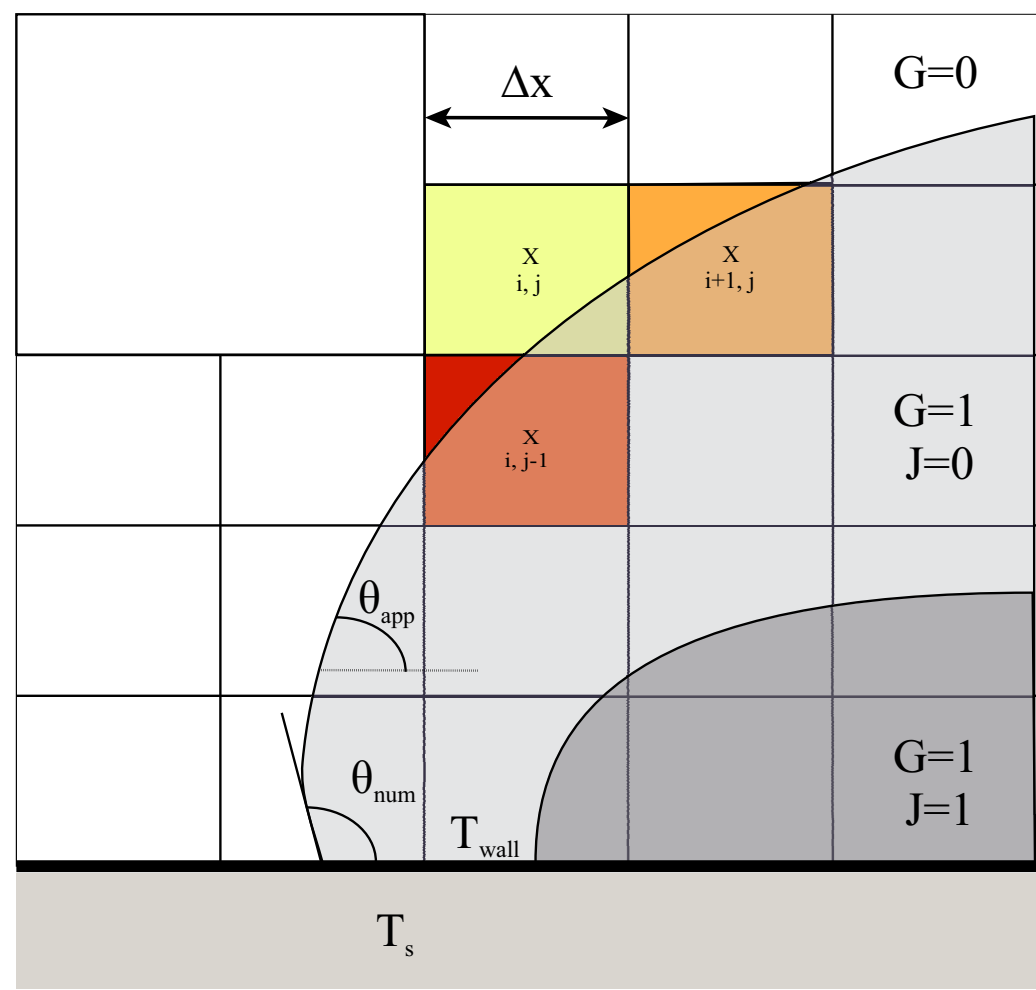

Figure 4.2: Mesh around a the interface of a droplet, with the apparent contact angle $\theta_{a p p}$ and imposed numerical contact angle $\theta_{\text {num }}$. Three phases are tracked during the impact: air $(G=0)$, liquid $(G=1, J=0)$ and solid $(G=1, J=1) . \Delta x$ is the (smallest) mesh size at the liquid-air and solid-liquid interface. 


\subsubsection{Heat transfer}

In general, the droplet is a combination of a liquid and a solid phase. The two phases and the liquid-solid front are determined after obtaining the solution of the heat equation, as shown in section 4.2.3. To take the latent heat $H_{f}$ of solidification into account for the droplet, the enthalpy equation is solved:

$$
\frac{(\partial \rho h)}{\partial t}+(\vec{u} \cdot \nabla) \rho h=\nabla \cdot(\lambda \nabla T),
$$

where $h(\vec{x}, t)$ is the enthalpy and $T(\vec{x}, t)$ is the temperature. The heat conductivity is denoted by $\lambda$. Enthalpy and temperature are directly related via the heat capacity $c_{p}$. For the droplet, the temperature is introduced as follows:

$$
T(\vec{x}, t)= \begin{cases}T_{m}+\frac{h}{c_{p}} & \text { for } h \leq 0 \\ T_{m} & \text { for } 0<h<H_{f} \\ T_{m}+\frac{h-H_{f}}{c_{p}} & \text { for } h \geq H_{f},\end{cases}
$$

where $T_{m}$ is the liquid melting temperature.

Using a single energy equation leads to spurious behaviour of the temperature field at the droplet-air interface. This can be understood from the coloured cells in Figure 4.2. A large difference in liquid fraction in adjacent cells can lead to a large difference in specific heats in these cells. We found that this gives rise to numerical dispersion near the liquid-gas interface. Therefore outside the droplet, we directly solve the energy equation, based on the temperature formulation:

$$
\frac{\partial\left(\rho c_{p} T\right)}{\partial t}+(\vec{u} \cdot \nabla)\left(\rho c_{p} T\right)=\nabla \cdot(\lambda \nabla T) .
$$

The temperature in the full domain is now calculated by applying equations (4.6) and (4.8) using the phase fraction at the interface:

$$
T=G T_{\text {droplet }}+(1-G) T_{\text {air }}
$$

\subsubsection{Solidification}

Solidification is taken into account implicitly through the energy equation for the droplet. The solid phase fraction and solid-liquid interface location is calculated from the values of the enthalpy inside the droplet. The solid phase fraction $J$ in terms of enthalpy is used in every numerical cell as:

$$
J(\vec{x}, t)= \begin{cases}0 & \text { for } h \geq H_{f} \\ \frac{H_{f}-h}{H_{f}} & \text { for } 0<h<H_{f} \\ 1 & \text { for } h \leq 0\end{cases}
$$




\subsection{Numerical model}

The fluid loses momentum through the sink term in the momentum equation. This sink term is based on the Carmen-Kozeny porous media approach [39] as previously used in solidification studies [31, 32, 40-42]. It can be expressed as:

$$
S_{j}=-C_{m u s h} \frac{J^{2}}{(1-J)^{3}+\varepsilon} \vec{u},
$$

where $J$ is the solid phase fraction and $\varepsilon$ a small number to prevent division by zero. The mushy zone constant $C_{\text {mush }}$, with units $\mathrm{Nsm}^{-4}$, determines the magnitude of damping. Increasing this value leads to an increased damping of the fluid flow. The constant can be written as $C_{m u s h}=5 \mu_{l} S_{f}^{2}$ [43], where $S_{f}$ is the total surface area based on the solid volume and $\mu_{l}$ is the viscosity of the liquid. Assuming the dendritic arms are slender cones, with base diameter $d_{d}, S_{f}$ can be approximated as $S_{f}=6 / d_{d}$ [44]. $d_{d}$ typically is of the order of $10^{-4} \mathrm{~m}$ [45], leading to typical values of $C_{\text {mush }}=10^{8} \mathrm{Ns} \mathrm{m}^{-4}$. Using the porous medium approach leads to a severe restriction in time stepping, depending on the values of $C_{m u s h}$ and $\varepsilon$, see appendix 4.A.3. In appendix 4.A.2 the influence of the magnitude of the technical parameters $C_{m u s h}$ and $\varepsilon$ on the droplet impact behaviour is investigated. In a certain range, robust behaviour is found, as require to make physical statements.

\subsubsection{Boundary conditions}

\section{Boundary conditions at the droplet-air interface}

The heat conductivity at the interface, where $0<G<1$, can be chosen to mimic a local heat resistance. Here the heat conductivity at the droplet-air interface is calculated using the harmonic mean. This prevents excessive heat conduction from the air towards the droplet. The value for the heat conductivity is then calculated as [46]:

$$
\lambda=\left(\frac{G}{\lambda_{p}}+\frac{1-G}{\lambda_{\text {air }}}\right)^{-1},
$$

where $\lambda_{p}$ is the heat conductivity of the droplet and $\lambda_{\text {air }}$ the heat conductivity of the air.

\section{Wall-droplet interaction}

It has been shown, that in GERRIS simulations, an impacting droplet entraps a small unphysical air layer, i.e. beyond the actual entrained air bubble [47-52], in the order of the mesh size below the droplet, when using no-slip boundary conditions [53]. Since droplet-surface contact must be resolved to account for the surface heat transfer, this must be overcome. In contrast to the direct removal of bubbles smaller than several cell sizes [54], here we use a slip length for the air flow. This model assumption leads 
to a faster outflow of air under the droplet. To limit this escape of air, the slip length is based on the local cell size as:

$$
u_{\text {wall }}=(1-G) \Delta x \frac{\partial \vec{u}}{\partial \vec{n}}
$$

Here $\Delta x$ is the local cell size at the surface and $\frac{\partial \vec{u}}{\partial \vec{n}}$ is the derivative of velocity in normal direction to the surface. For the phase fraction $G=0$, the slip length equals the cell size. For the phase fraction $G=1$, the no-slip boundary condition is obtained.

The numerical dynamic contact angle model of Ref. [55] has been used to allow the contact line to move along the no-slip boundary:

$$
\cos \theta_{\text {num }}=\cos \theta_{\text {app }}+5.63 C a \ln K / \delta,
$$

where $\theta_{\text {num }}$ is the numerical contact angle, $\theta_{\text {app }}$ the apparent contact angle, Ca the capillary number $C a=\mu U / \sigma$ and the constant $\mathrm{K}$ depends on the slip model and outer region length scale. Here $K=0.02$, and $\delta=\Delta x / 2$ as described in Ref. [55].

Due to imperfections in two touching surfaces, a difference in temperature between the two touching bodies can occur [56]. This thermal contact resistance also occurs for molten metal droplets impacting on surfaces [1,27,36, 57], e.g. due to the roughness of the surface [58]. To account for thermal contact resistance between the surface and the droplet, the heat transfer from the surface to the droplet is set by a mixed type boundary condition. At a distance from the surface the temperature is prescribed with $T_{s}$, see figure 4.2. The boundary condition can be written as [59]:

$$
T_{\text {wall }}=T_{s}+R \lambda \frac{\partial T}{\partial \vec{n}}
$$

Here $R\left(\mathrm{~m}^{2} \mathrm{KW}^{-1}\right)$ is the thermal contact resistance between the surface and the droplet. This physical parameter is previously used to fit a solidification model [26]. Its effect is studied in section 4.3.2. $T_{\text {wall }}$ is the temperature at the contact surface. If there is no thermal contact resistance, $R=0$, the boundary condition reduces to a Dirichlet type boundary condition, where $T_{\text {wall }}=T_{s}$. 
4.3. Validation \& sensitivity for impact of tin droplets
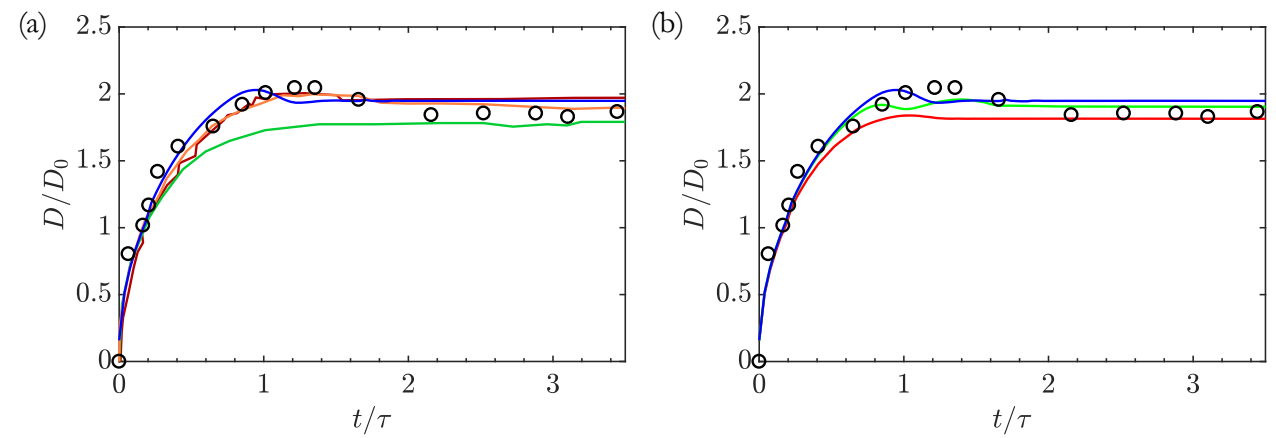

Figure 4.3: Spread factor of droplets versus time. (a) Comparison with other numerical methods: ○: experimental results [57], — :[30], — : [26], — : [32], — : current model. All numerical methods use $R=5 \cdot 10^{-6} \mathrm{mKW}^{-1}$.

(b) Various values of contact resistance $R$ : ०: experimental results [57], $-: R=0 \mathrm{mKW}^{-1}$, $: R=2.8 \cdot 10^{-6} \mathrm{~m} \mathrm{KW}^{-1}, \ldots$ : $R=5 \cdot 10^{-6} \mathrm{mK} \mathrm{W}^{-1}$.

\subsection{Validation \& sensitivity for impact of tin droplets}

Table 4.1: Material properties, taken from Ref. [60]

\begin{tabular}{lll}
\hline Material & $\begin{array}{l}\text { Tin } \\
(\text { droplet })\end{array}$ & Air \\
\hline Density $\rho$ (kilogram $\left./ \mathrm{m}^{3}\right)$ & 6980 & 1.2 \\
Viscosity $\eta$ (pascals) & $1.5 \times 10^{-3}$ & $1.8 \times 10^{-5}$ \\
Surface tension $\sigma$ (newton $/ \mathrm{m})$ & 0.514 & - \\
Thermal Conductivity $\mathrm{k}(\mathrm{watt} / \mathrm{m} / \mathrm{K})$ & 67 & 0.023 \\
Heat capacity $c_{p}($ joule $/ \mathrm{kg} / \mathrm{K})$ & 210 & 1000 \\
\hline
\end{tabular}

In this section, the numerical method is validated by comparing it to the experiments performed by Ref. [57] and to earlier numerical methods that used the same experiments as validation [26, 30, 32]. In the work of Ref. [57] a spherical droplet of tin with diameter $2.7 \mathrm{~mm}$ was impacting onto a substrate with a velocity of $1 \mathrm{~m} \mathrm{~s}^{-1}$. The substrate had a temperature of $20^{\circ} \mathrm{C}$. The droplet initial temperature was $240{ }^{\circ} \mathrm{C}$ with a solidification temperature of $232^{\circ} \mathrm{C}$.

For the numerical simulation, the physical properties of the materials are shown in table 4.1. The sensitivity of the numerical model to the parameters $C_{m u s h}$ and $\varepsilon$, as well as a mesh sensitivity study, can be found in appendix 4.A. 


\subsubsection{Comparison to earlier models}

In figure 4.3a the dimensionless spreading $D / D_{0}$ versus dimensionless time $t / \tau$ is shown, for the experiments of Ref. [57], the current model and various earlier numerical models. Here $\tau$ is the inertial time scale $\tau=D_{0} / V_{0}$. The earlier models that have been used are: firstly fixing the velocity during solidification [26], secondly a penalty method where the viscosity is increased based on a porous medium approach [32], and thirdly a penalty method with linear increase of viscosity as a function of solidified fraction [30]. For comparison of the current model to earlier models, the same value for the contact resistance of is used: $R=5 \cdot 10^{6} \mathrm{~m}^{2} \mathrm{KW}^{-1}$.

All numerical models follow the same trend of the spreading phase of the droplet as the experiment for $t / \tau<0.5$. The spreading in the current method, after $t / \tau=0.5$ is slightly larger than that of the previously developed methods. The current method is the only one that shows the contact line increasing up to the maximum spreading diameter that was observed in the experiment. After $t / \tau>1$, the experiments show significant retraction of the contact line before pinning. Only the mushy zone models of Ref. [32] and the current model show this behaviour. Finally, the model of Ref. [30] under-predicts the final droplet diameter. In contrast, the current model shows a final droplet diameter that is slightly larger than that observed in the experiments. The model of Ref. [32] predicts almost the same spreading diameter as the current model, while the model of Ref. [26] shows a final droplet diameter closer to that of the experiments. It should be noted, that the model by Ref. [26] used the value of the contact resistance as a fitting parameter, while the other models follow their initial assessment of the value of $R=5 \cdot 10^{6} \mathrm{~m}^{2} \mathrm{KW}^{-1}$. The current model and the model by Ref. [32] perform the best to show the general behaviour of spreading and retraction of the droplet. The influence of the contact resistance on model behaviour is investigated in the next section.

\subsubsection{Effect of the contact resistance}

The surface contact resistance was shown to have a strong influence on the spreading behaviour of droplets in Ref. [35]. The contact resistance of $R=2.8 \cdot 10^{6} \mathrm{~m}^{2} \mathrm{KW}^{-1}$ was calculated for the experimental results of Ref. [57], based on the measured time dependant surface temperature. A best match for the maximum spreading diameter of the numerical model of Ref. [26] was found using a contact resistance of $R=5 \cdot 10^{6} \mathrm{~m}^{2} \mathrm{KW}^{-1}$. This contact resistance was also used by Ref. [32], Ref. [30], and in the current method in the previous subsection. Using a lower contact resistance obviously increases the solidification rate, leading to a lower spreading rate.

The effect of the contact resistance on the behaviour of the current model can be seen in figure 4.3b. Setting the value of the thermal contact resistance to $R=0$ under-predicts the final droplet diameter and diminishes the effect of retraction of 


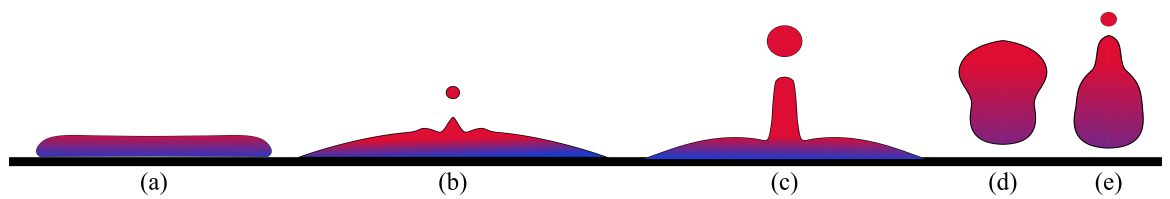

Figure 4.4: Types of outcome for a droplet impacting on a cooled surface. (a) Deposition. (b) Capillary pinch-off. (c) partial rebounce. (d) Rebounce. (e) Rebounce with ejection. The colors are an artist impression of the cooling of the droplet.

the droplet. Using the heat resistance determined experimentally in Ref. [57] of $R=2.8 \cdot 10^{6} \mathrm{~m}^{2} \mathrm{KW}^{-1}$ shows both the spreading and retraction behaviour before pinning as is also seen in the experiment. Furthermore, it leads to a prediction of the final droplet diameter closer to the experimental results than the higher contact resistance used in earlier methods. Summarizing, our method is the most similar to the experimentally observed spreading behaviour using the originally proposed contact resistance of $R=2.8 \cdot 10^{6} \mathrm{~m}^{2} \mathrm{KW}^{-1}$.

\subsection{Results}

We now focus on impact with solidification of $1 \mathrm{~mm}$ tin droplets, using a range of velocities $\left(0.1\right.$ to $2 \mathrm{~m} \mathrm{~s}^{-1}$, corresponding to $0.1<\mathrm{We}<55$ ) and temperatures ( 25 to $235{ }^{\circ} \mathrm{C}$, corresponding to $0<$ Ste $<0.75$ ). The surface is assumed to be perfectly phobic to tin ( i.e. $\theta_{s}=180^{\circ}$ ). To account for the damping term in the momentum equation (see section 4.2.3), a secondary dendrite arm size of $d_{d}=40 \mu \mathrm{m}$ was used, as it lies in the range of results of earlier models and experimental results [61-63], leading to $C_{m u s h}=1.7 \cdot 10^{8} \mathrm{~N} \mathrm{~s} \mathrm{~m}^{-4}$. For consideration of time stepping (see section 4.A.3), the value of $\varepsilon=10^{-3}$ was chosen. Further information on the sensitivity of the model to these parameters is given in appendix 4.A.2.

\subsubsection{Regime map}

Depending on the control parameters, five different scenarios can occur, as qualitatively shown in figure 4.4. Time series of the impact event, for a constant impact velocity of $U=1.4 \mathrm{~m} \mathrm{~s}^{-1}$ and various temperatures, are shown in figure 4.5 . Furthermore outcomes for a constant substrate temperature of $T_{s}=150^{\circ} \mathrm{C}$ and various impact velocities are shown in figure 4.6. During solidifying after droplet impact, various phases can be observed. First the droplet starts to spread over the surface. Solidification already starts to occur from the contact area of the droplet with the surface. Air is entrained between the droplet and the surface. As it cannot escape, small bubbles are observed inside the droplet [47-53]. During the spreading of the droplet capillary waves form at the interface $[64,65]$. At a certain moment in time, the droplet has 


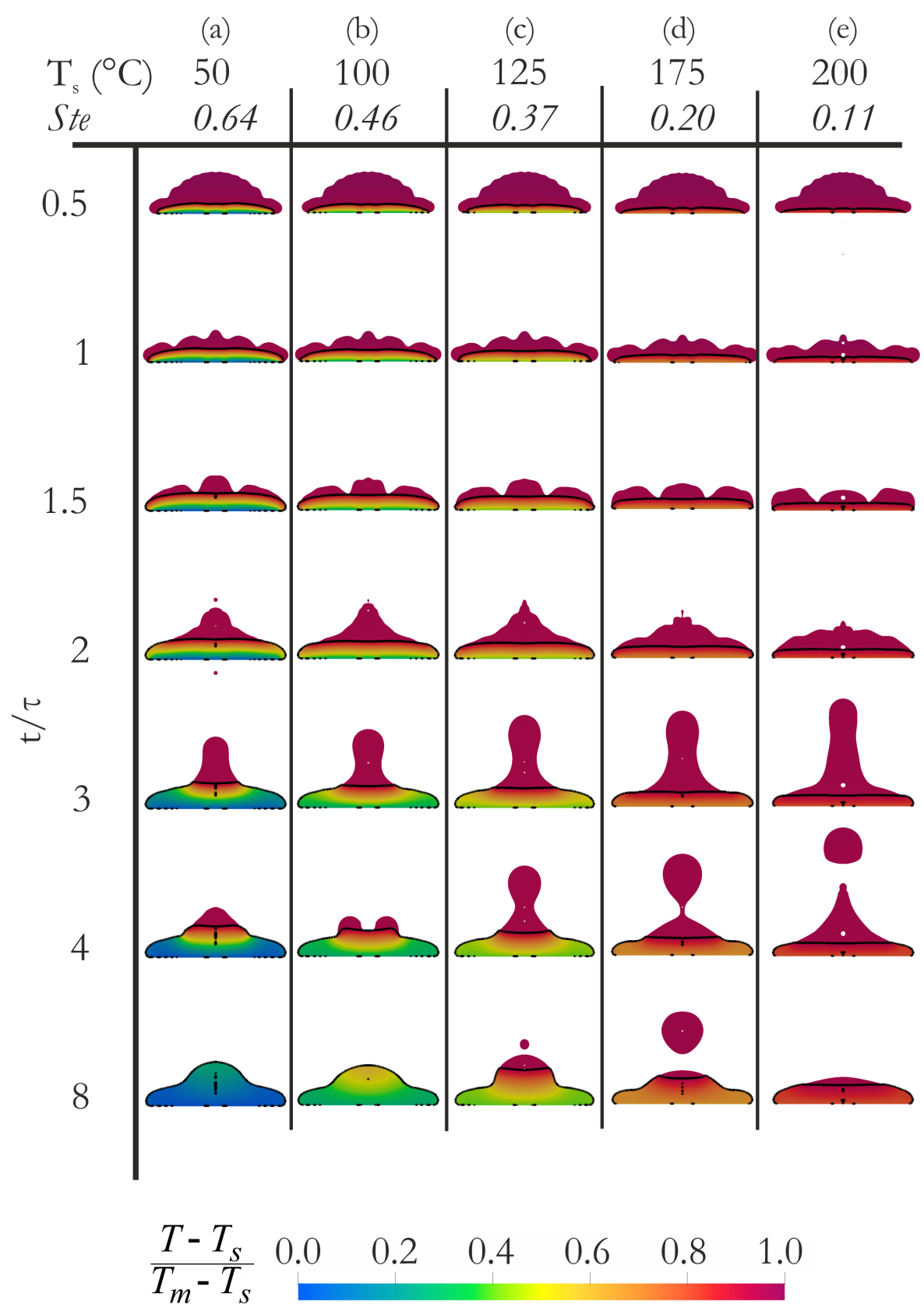

Figure 4.5: Time series of drop impact on a surface with $U=1.4 \mathrm{~m} \mathrm{~s}^{-1}$. From left to right different surface temperatures are shown. —-shows the solidification front. Movies of the times series are available in the supplementary material. 


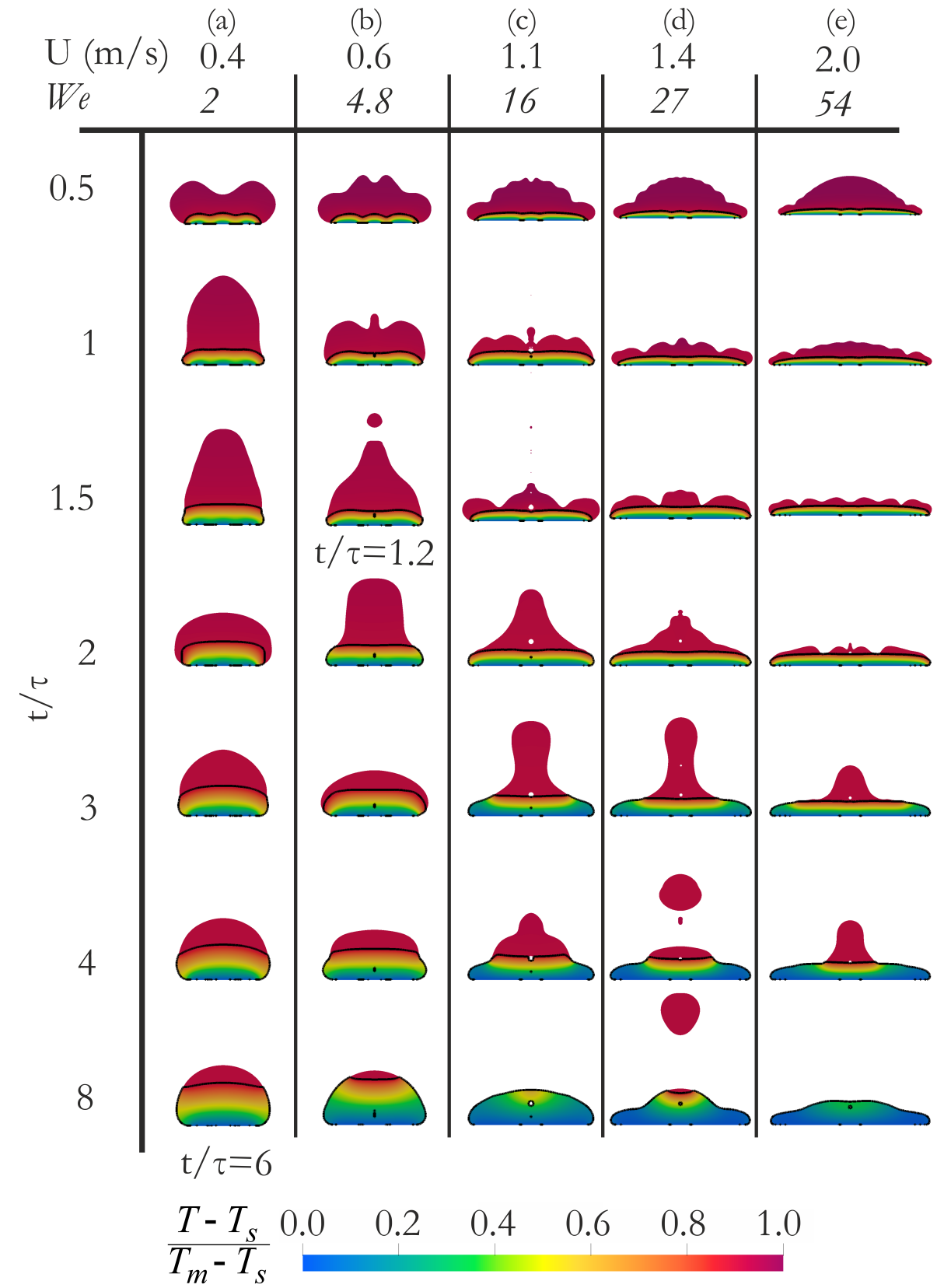

Figure 4.6: Time series of drop impact on a surface with $T_{s}=150^{\circ} \mathrm{C}$, Ste $=0.28$. From left to right different impact velocities are shown. —-shows the solidification front. The snapshots labelled with $(*)$ are taken at a different $t / \tau$ to highlight the behaviour. Movies of the times series are available in the supplementary material. 


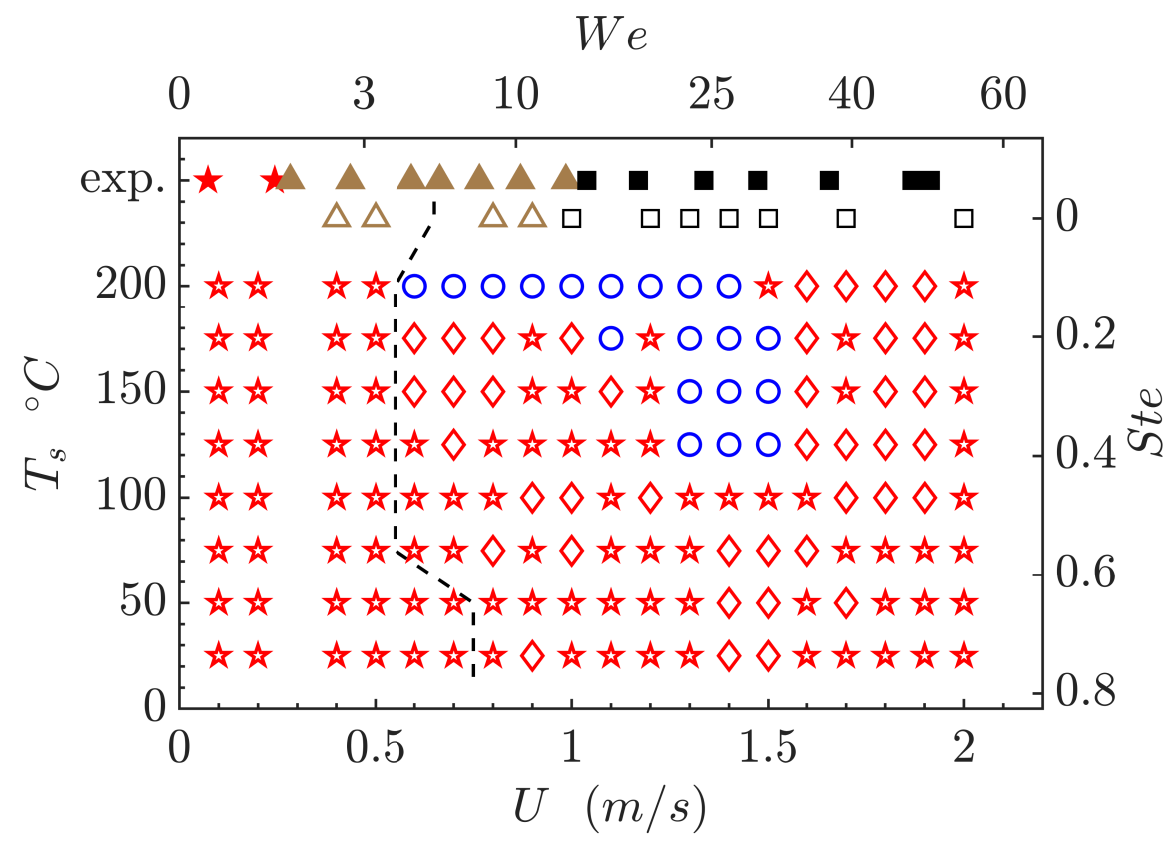

Figure 4.7: Phase diagram showing the outcome of droplet impact as a function of velocity (or We) and temperature (or Ste). The impact behaviour observed are deposition ( $)$, rebound $(\triangle)$, rebound with ejection $(\square)$, partial rebound $(\circ)$ and capillary ejection $(\diamond)$. For all cases to the right of the dashed line (- - ) a bubble is entrapped in the droplet. Closed symbols indicate isothermal experiments of Ref. [67].

reached its maximum spreading diameter. After this it starts retracting due to capillarity. During this retraction phase, part of the droplet can detach from the main droplet. Two mechanisms have been identified:

- First, the oscillations of the capillary waves travels from the contact line region towards the top of the drop, leading to a pinching of a small amount of material at the center of the droplet [65], e.g. figure 4.6, with $U=1.1 \mathrm{~m} \mathrm{~s}^{-1}$, or figure 4.5, with $T_{s}=50^{\circ} \mathrm{C}$.

- Second, after retraction of the droplet on the surface, a vertical column of liquid rises up in the centre of the droplet, which leads to either necking of the thin liquid column, e.g. figure 4.6, with $U=0.6 \mathrm{~m} \mathrm{~s}^{-1}$, or figure 4.5 , with $T_{s}=$ $200^{\circ} \mathrm{C}$; or an intense recoil, e.g. figure 4.6 , with $U=1.4 \mathrm{~m} \mathrm{~s}^{-1}$, or figure 4.5 , with $T_{s}=175^{\circ} \mathrm{C}$. This leads to break-up of the droplet into two parts

Furthermore, the capillary waves can collapse in the centre of the droplet and enclose an air bubble in the droplet [66], which in this case is later frozen inside the droplet. 
The general behaviour is shown in a phase diagram (figure 4.7), in the five distinct types of behaviour, already shown in figure 4.4: deposition, capillary ejection of a droplet, (partial) rebounce with and without ejection. The phase diagram extends the phase diagram for isothermal droplet deposition $(\mathrm{Ste}=0)$, determined experimentally by Ref. [67]. For the isothermal case, the simulated results agree reasonably well with previously obtained results. A transition from the deposition to rebound is seen between $0.5<\mathrm{We}<2$, when the remaining kinetic energy in the droplet is sufficiently high to overcome surface and potential energy [67]. A transition into the rebound with ejection regime, when even higher kinetic energy remains in the retracting droplet, is seen between $11<\mathrm{We}<13.5$ here and at $\mathrm{We}=14$ in the experiments by Ref. [67]. The slight difference might be due to the slightly lower contact angle of $150^{\circ}$ in the experiments.

We will now discuss the competition between the spreading and solification, with increasing Stefan number.

- First, at slight surface undercooling, $0.1<$ Ste $<0.4$, similar to isothermal impact, for We $<4$, complete deposition of the droplet on the surface takes place. This happens at $\mathrm{We}<2$ in the isothermal case [67]. There is not enough inertia for (partial) re-bounce to occur. For $\mathrm{We}>4$, a liquid column rises at the centre of impact after retraction of the droplet (figure 4.4c). Independent of impact velocity, this occurs around $0.4 t_{c}$, where $t_{c}=2.6\left(\rho D_{0}^{3} / \sigma\right)^{1 / 3}$. The droplet behaves similar to an elastic spring, although the behaviour inside the drop is non-linear [68]. The solidification front has no influence on the free surface behaviour of the droplet in this case. During the rise of the column, a droplet is launched due to the capillary instability of this column (partial re-bounce scenario). A transition is seen towards deposition, where the capillary instability does not break-off a droplet any more, for We $>50$.

- Second, for $0.2<$ Ste $<0.4$, the neck of the droplet does not pinch-off for all Weber numbers. However, the behaviour of the droplets after impact is still very sensitive to the impact Weber number. The transition from deposition through partial re-bounce back to deposition can be understood from the solidification rate of the droplet. Figure 4.8a shows the relative solidified volume versus time for various impact velocities for $\mathrm{Ste}=0.28$. Note that, for $\mathrm{We}=27$ the freezing is limited, due to the pinch off of a secondary droplet. The higher the Weber number, the larger the contact area, which the droplet has with the surface. Kinetic energy is lost at the solidified layer. This leads to a higher solidification rate. Due to the large contact area and thus faster solidification at higher We, the kinetic energy during the rebounce fase is too low for a droplet to pinch off, so deposition takes place. Ref. [69] proposed a model based on the estimation of excess energy at the moment of rebounce. If the rebounce energy 

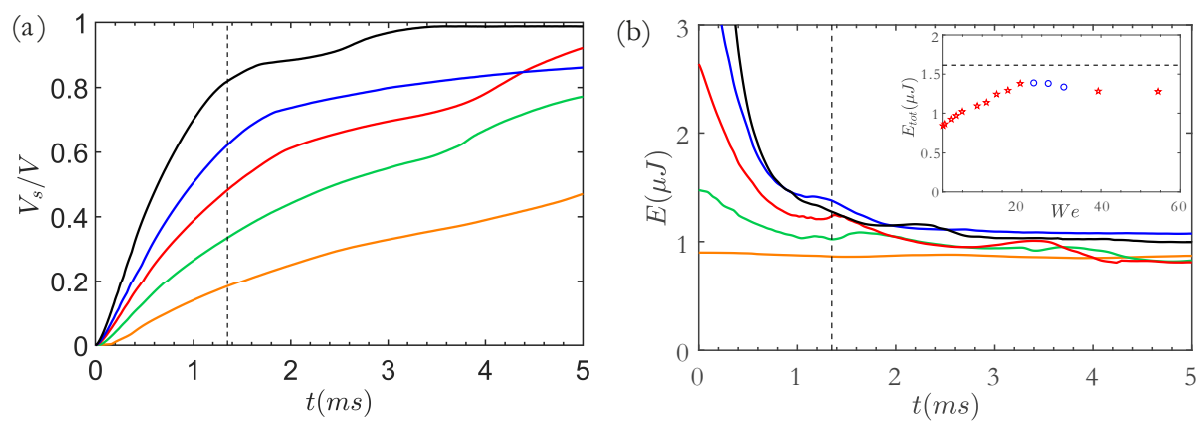

Figure 4.8: (a) Solidified volume and (b) energy as a function of time for various velocities, for $\mathrm{Ste}=0.28$. $\mathrm{We}=0.5(-), \mathrm{We}=5(-), \mathrm{We}=13(-), \mathrm{We}=27(-), \mathrm{We}=54$ (-). The dashed line - - -shows the moment the column starts to rise, at $0.4 t_{c}=1.38 \mathrm{~ms}$. The inset shows the total energy at $0.4 t_{c}$ as a function of We. The markers are as those in 4.7 : deposition (*), partial rebound (o). The dashed line in the inset is the free surface energy of a tin droplet of $D_{0}=1 \mathrm{~mm}$.

was higher than the necessary energy to create a pinched-off droplet surface, rebounce occurs. However, in our case, the differences in energy of the various impact scenarios at the start of the column rise are minute (see figure $4.8 \mathrm{~b}$ ), hence for these intermediate Weber numbers, the precise effect leading up to the transition needs to be further investigated. A detailed study of the behaviour of the rising column is necessary to fully understand (partial) rebounce.

- Finally, at sufficiently high surface undercooling, Ste $>0.5$, independent of Weber number, the droplet freezes directly onto the surface. The solidification front grows quickly enough to dampen all droplet momentum and keep the droplet from bouncing off the surface.

\subsubsection{Effect of We and Ste on droplet spreading}

Figure 4.9a shows the isothermal spreading diameter $D(t)$ as a function of time for droplets with various impact velocities. Figure $4.9 \mathrm{~b}$ shows the same information, but on a log-log scale and rescaled. From that plot, one can easily see that the spreading lamella, follows an empirical relation, obtained from geometrical considerations and mass conservation :

$$
\frac{D_{c}}{D_{0}} \approx 2.9\left(\frac{t}{\tau}\right)^{1 / 2},
$$

[70] until surface tension effects lead to retraction of the interface.

The influence of solidification on the spreading dynamics for the same Weber numbers is shown in figure 4.10 for various Stefan numbers. 

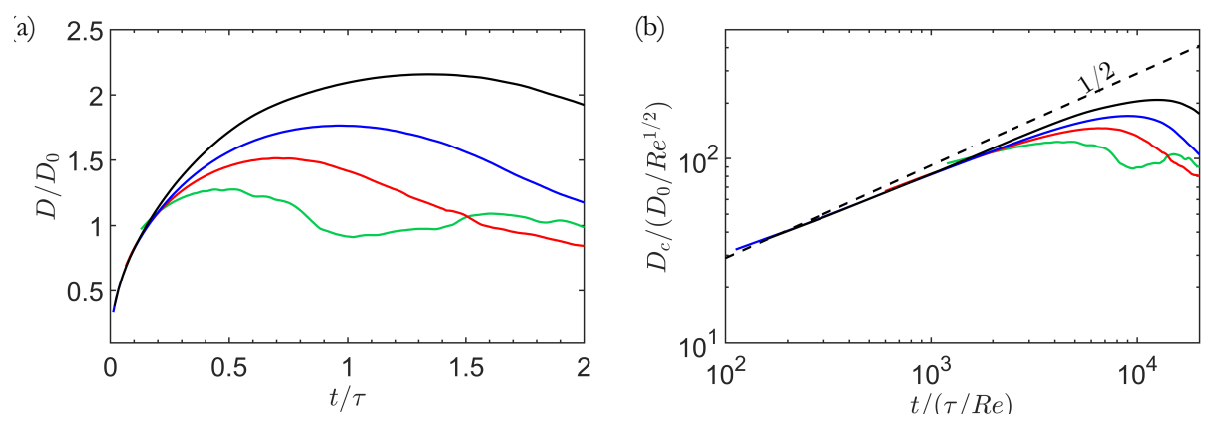

Figure 4.9: Dimensionless spreading versus time with $T>T_{m}$ for different Weber numbers $\mathrm{We}=2.1(-), \mathrm{We}=8.7(-), \mathrm{We}=16(-), \mathrm{We}=39(-)$. (a) Droplet maximum diameter versus time (b) Dimensionless spreading of the contact diameter $D_{c} / D_{0}$ normalised by $\operatorname{Re}^{1 / 2}$ versus $t / \tau$, normalised by Re. For small times $t / \tau$, the numerical model agrees well with the scaling law proposed by Ref. [70], equation (4.16), shown by the dashed line (- - -).
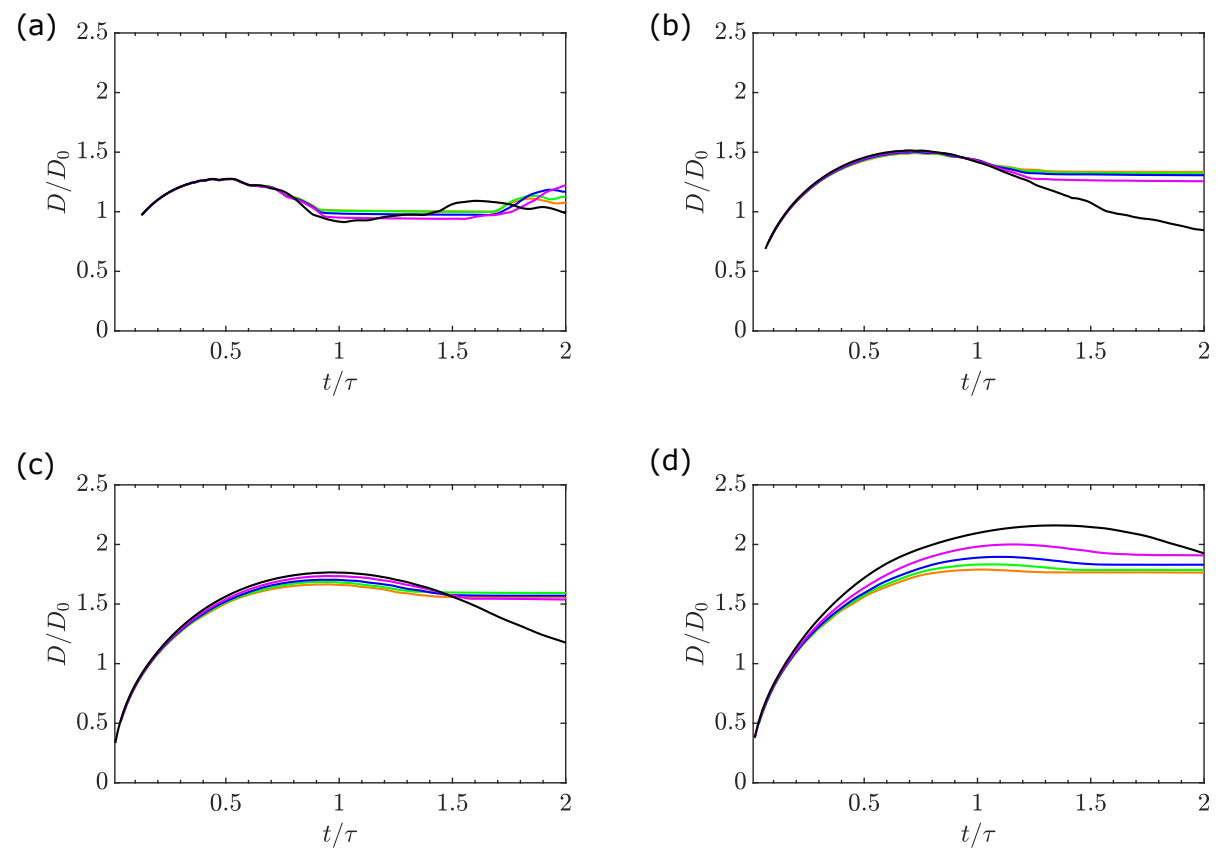

Figure 4.10: Dimensionless spreading of the droplet as a function of time for We $=2.1$ (a), $\mathrm{We}=8.7(\mathrm{~b}), \mathrm{We}=19(\mathrm{c})$, and $\mathrm{We}=39(\mathrm{~d})$ and various initial temperatures $\left(T_{s}=50^{\circ} \mathrm{C}\right.$ $\left.(\sqcup), T_{s}=100{ }^{\circ} \mathrm{C}(\sqcup), T_{s}=150{ }^{\circ} \mathrm{C}(-), T_{s}=200{ }^{\circ} \mathrm{C}(-), T_{s}>T_{m}(-)\right)$

- First, for low Weber number impacts (We $\lesssim 10$ ), the shape of the spreading droplet resembles a spherical cap. During the contact time with the surface, the solidification front is far away from the free surface. The droplets spreading 
with solidification is similar to impact without solidification, up to the maximum spreading diameter, after which surface tension leads to retraction of the interface. The position of the solidification front lags behind the contact line position. During the retraction phase the liquid flows over the previously solidified zone, and the maximum horizontal position of the solidification front determines the final arrest diameter.

- Second, at intermediate Weber numbers (We $\gtrsim 19$ ), figure 4.10c, the solidification has a small influence on the spreading behaviour, when the droplet extends near the maximum spreading diameter. The maximum spreading diameter is slightly diminished due to the solidification.

- Third, at even higher Weber numbers (We $\gtrsim 30$ ), figure 4.10d the solidification slows down the spreading rate much earlier. The droplet resembles a thin liquid cylinder during spreading, figure 4.4a. Due to the high spreading diameter, the height of the lamella is only slightly larger than the height of the solidification front. This leads to complete solidification of the lamella. For this case, the maximum droplet diameter for solidifying droplets is reached earlier than for the isothermal droplet, and only the rim slightly retracts towards the solidified front.

\subsubsection{Maximum spreading diameter}

We now explain the effect of solidification on the maximum spreading diameter. Figure 4.11 shows the maximum spreading diameters for various impact velocities and temperatures.

For isothermal spreading, for $\mathrm{We}<1$ the maximum spreading diameter follows the elastic regime behaviour of small deformations, for a free slip-surface, independent of surface temperature. The kinetic energy is transformed into surface energy $[72,74,75]$, and the energy loss due to solidification has no significant influence on the maximum spreading diameter. For the maximum spreading rate, $\tilde{D}_{m}-1=\sqrt{5 / 96 \mathrm{We}}$ is found [74], with the maximum dimensionless spreading diameter $\tilde{D}_{m}=D_{m} / D_{0}$.

For higher Weber numbers, We $\gtrsim 10$, the maximum spreading diameter of isothermal spreading, $T_{0}>T_{m}$, follows the scaling law of Ref. [72], based on the energy balance between the initial kinetic and surface energy, equated with the surface energy at maximum droplet spreading and the dissipation:

$$
\frac{3(1-\cos \theta) \tilde{D}_{m}^{2}}{\mathrm{We}}+\frac{0.6 \tilde{D}_{m}^{2} \sqrt{\tilde{D}_{m}-1}}{\sqrt{\mathrm{Re}}}=\frac{12}{\mathrm{We}}+\frac{1}{2} .
$$



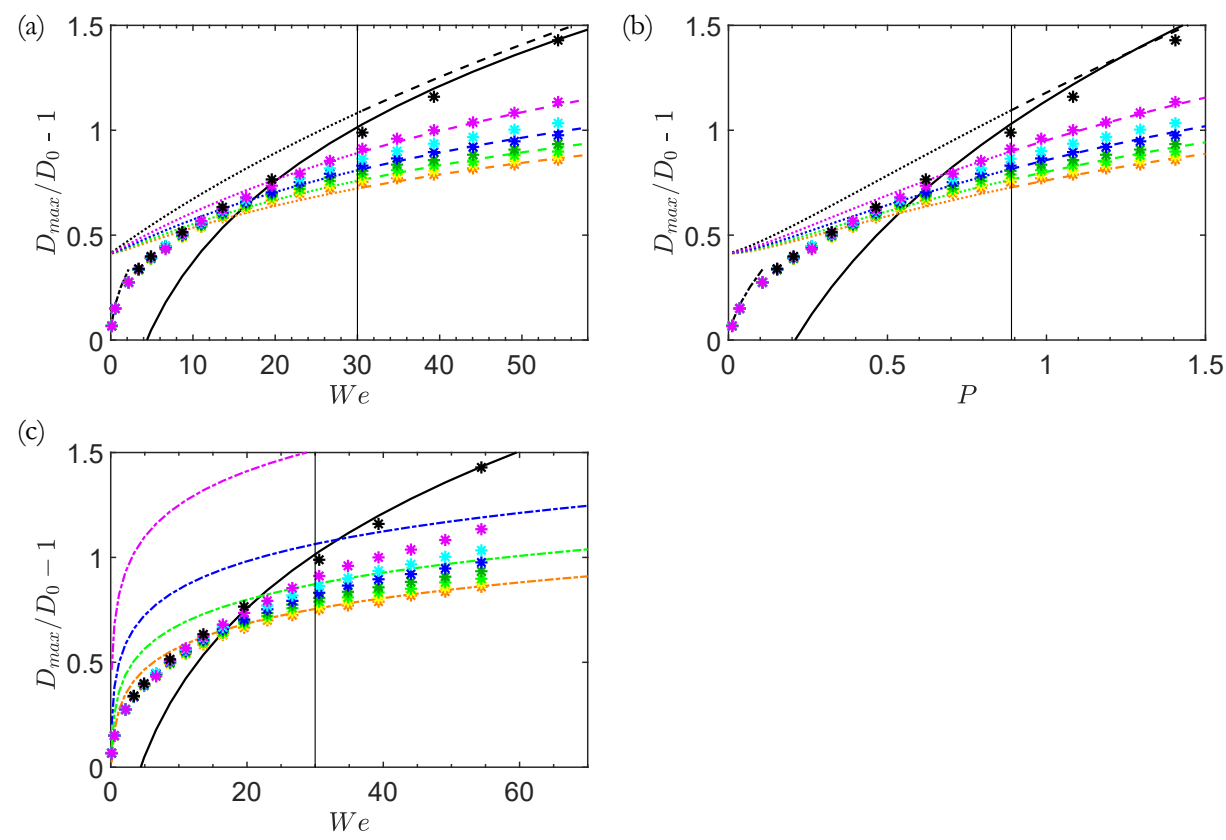

Figure 4.11: Maximum spreading of the droplets against for various initial temperatures $\left(T_{s}=50{ }^{\circ} \mathrm{C}(\right.$ (*) $), T_{s}=100{ }^{\circ} \mathrm{C}\left(\right.$ *) $, T_{s}=150{ }^{\circ} \mathrm{C}(*), T_{s}=200{ }^{\circ} \mathrm{C}($ * $), T_{s}>T_{m}$ (*)). (a) Against We and (b) against impact parameter $P \equiv \mathrm{WeRe}^{-2 / 5}$. The black line (—) shows equation (4.18) proposed by [71], with a fitting constant of $A=1.785$. The dashed black line (- - ) shows the model of Ref. [72], equation (4.17). Although strictly only valid for We $>30$, it also predicts the maximum spreading down to We $\gtrsim 10$, shown by the dotted line $(\cdots \cdots)$. The coloured dashed lines show equation (4.23), with temperatures corresponding the coloured stars. (c) Against We, with the black line (-) showing equation (4.18) proposed by Ref. [71] and the coloured dashed lines showing equation (4.25), proposed by Ref. [73]. The temperatures correspond to the coloured stars. (c)

Ref. [71] proposed a scaling law as a crossover between two asymptotic regimes based on the impact parameter $P \equiv \mathrm{WeRe}^{-2 / 5}$. The crossover between the capillary regime, $P<<1$, and the high-viscous regime, $P>>1$, can be parametrised as:

$$
\frac{D_{\max }}{D_{0}} \operatorname{Re}^{-1 / 5}=\frac{P^{1 / 2}}{A+P^{1 / 2}},
$$

where $A$ is a fitting constant. In the numerical calculations performed here, $P=\mathscr{O}(1)$, which is in the crossover between the two asymptotic regimes. Figure $4.11 \mathrm{~b}$ shows the maximum diameter as a function of the impact parameter $P$. Our data agrees well with the model of Ref. [71], for We $\geq 20$ ( $P \geq 0.6$ ), using $A=1.785$, which shows that also for tin droplets, the crossover can be well described by equation (4.18). 


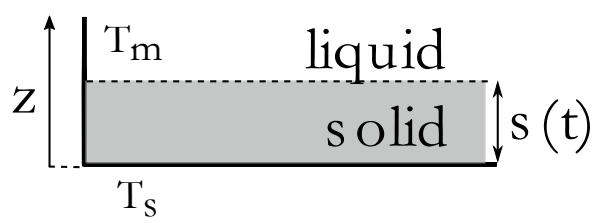

Figure 4.12: Schematic view of a one-dimensional solidification process. A solidified layer with thickness $s(t)$ grows on top of a mould that is kept at constant temperature $T_{s}$. The melting temperature of the liquid is given as $T_{m}$.

The effect of the solidification on the maximum spreading diameter is clearly visible, for the higher Weber numbers. To describe the maximum spreading extent for a solidifying droplet, we start from the scaling law of Ref. [72], equation (4.17), which describes the maximum spreading extent for the isothermal case. To add the effect of solidifaction to this scaling law, we take the idea of Ref. [23], which assumes that the kinetic energy in the layer that solidifies is dissipated. As the complex interaction between the fluid motion and the solidified layer is not fully described by this simplified assumption, we use a fitting parameter $a$. Hence, if the solid layer has volume $V_{s}$, the kinetic energy loss can be described with $\Delta E_{k i n} \approx V_{s}\left(a / 2 \rho U_{0}^{2}\right)$. The solidified volume is calculated from the simplified assumption that heat transfer occurs by one-dimensional heat conduction, see figure 4.12, and that one has small Stefan number, Ste $=C_{p}\left(T_{m}-T_{s}\right) / H_{f}<1$, such that the heat transfer can be assumed to be quasi-steady. The dynamics of the solidification front is then given by [76]:

$$
\rho H_{f} \frac{\delta s}{\delta t}=-\lambda \frac{T_{m}-T_{s}}{s} .
$$

The thickness of the solid layer at any position $\tilde{s}=s / D_{0}$ then increases with dimensionless time as:

$$
\tilde{s} \sim \sqrt{\frac{t}{\tau} \frac{\mathrm{Ste}}{\mathrm{Pe}}}
$$

The solidification at a certain spreading distance starts immediately after the liquid is in contact with the surface at a delay time $t_{d}$. Using equation (4.16) for the spreading of the contact diameter, the delay time scales with $t_{d} \sim \tilde{D}^{2}$. Including the delay time in equation (4.20), the solidification height above any spreading distance can be written as:

$$
\tilde{s} \sim \sqrt{\left(\frac{t}{\tau}-\frac{t_{d}}{\tau}\right) \frac{\mathrm{Ste}}{\mathrm{Pe}}}
$$




\subsection{Results}

The dimensionless solidified volume $\tilde{V}_{s}=V_{s} / V_{0}$ can be found by integrating this equation, using equation (4.16) up to the maximum spreading diameter, as:

$$
\tilde{V}_{s} \sim \int_{0}^{\tilde{D}_{\max }} \int_{0}^{\pi} \sqrt{\left(\tilde{D}_{\max }^{2}-\tilde{D}^{2}\right) \frac{\mathrm{Ste}}{\mathrm{Pe}}} \tilde{D} d \theta d \tilde{D}=\pi / 3 \sqrt{\frac{\mathrm{Ste}}{\mathrm{Pe}}} \tilde{D}_{\max }^{3}
$$

Hence, the loss of kinetic energy is approximated by:

$$
\frac{\Delta E_{k i n}}{E_{k 0}} \sim a \tilde{D}_{\max }^{3} \sqrt{\frac{\mathrm{Ste}}{\mathrm{Pe}}} .
$$

Combining equations (4.17) and (4.23) gives an estimate of the maximum spreading diameter of a solidifying droplet:

$$
\frac{3(1-\cos \theta) \tilde{D}_{m}^{2}}{\mathrm{We}}+\frac{0.6 \tilde{D}_{m}^{2} \sqrt{\tilde{D}_{m}-1}}{\sqrt{\mathrm{Re}}}+a \pi / 3 \tilde{D}_{\max }^{3} \sqrt{\frac{\mathrm{Ste}}{\mathrm{Pe}}}=\frac{12}{\mathrm{We}}+\frac{1}{2}
$$

The maximum spreading diameters and the results of equation (4.23) are shown in figure 4.11. Using a fitting parameter of $a \approx 0.4$ gives a good prediction of the maximum spreading diameter. Although both the hydrodynamic spreading and the effect of solidification give rise to complex spreading behaviour, this relatively simple model is a useful tool to predict maximum droplet spreading for freezing droplets.

A different approach to predict the maximum spreading diameter for solidifying droplets has been proposed by Ref. [73]. Here the calculated thickness of a solidified layer is compared to the center height of a spreading pancake. Once the thickness reaches the center height, no more liquid can spreading towards the contact line and the maximum spreading diameter is obtained. The maximum diameter is then given by:

$$
\tilde{D}_{\max }=\left(\frac{\mathrm{Pe}}{\text { Ste }}\right)^{1 / 5}
$$

Figure 4.11c shows the outcome with this model compared to the numerical calculations. In the regime studied, this model predicts the maximum spreading diameter very accurately for the complete range of We, for the lowest surface temperature $\left(T_{s}=50\right.$ $\left.{ }^{\circ} \mathrm{C}\right)$. For higher temperatures, the model overpredicts the maximum spreading diameter. For the We numbers studied here, the assumption of a flat pancake is not valid. Since for these higher temperatures the solidified layer grows significantly slower than for the low temperature case, the maximum droplet spreading has already been reached due to visco-capillary effects, which are not included in the model of Ref. [73]. For the We $>10$ numbers studied here, equation (4.24) gives a good prediction for the maximum droplet diameter for solidifying droplets, while in the regime of high We numbers, where the assumption of a flat pancake is valid, the maximum droplet diameter can converge to equation (4.25). 


\subsection{Conclusions and Outlook}

Solidification has a large influence on droplet spreading and deposition behaviour. A numerical study was presented based on an incompressible multi-phase numerical model. The VoF method was used to track the free surface, using height functions to accurately calculate the curvature as implemented in GERRIS. An enthalpy-porosity method was implemented to account for heat transfer and solidification. The use of dynamic mesh refinement with a well-balanced surface tension description, leads to a favourable validation of the numerical method against earlier models and experimental data for tin droplet impact.

This method was then used to study droplet impact with simultaneous solidification, for tin droplets of $1 \mathrm{~mm}$, impacting with $0.1<W e<55$. An extension of the isothermal regime diagram for droplet impact on supercooled surfaces shows a wide array of behaviour. For high Stefan numbers, the droplets solidify immediately during the spreading phase, and no re-bounce or capillary ejection takes place. For low Stefan numbers a transition is seen from deposition for low Weber numbers, towards partial re-bounce. For larger Weber numbers a second transition is observed, back to deposition, in contrast to iso-thermal impact, where more violent rebounce events occur. This difference is due to the energy loss imposed by the solidification.

The early spreading behaviour of solidifying droplets is similar to that of droplets impacting on a surface above the melting temperature of the liquid. For $W e<10$, only in the retraction phase solidification starts having an effect. For $W e>10$ solidification leads to a smaller maximum spreading diameter than predicted by theory based on isothermal droplet impact. To show this effect, a new model based on energy conservation arguments is proposed to predict the maximum droplet spreading including solidification effects. With a single fitting parameter, this model is shown to predict maximum droplet diameters for freezing droplets, for $W e>10$. For the studied $W e$ numbers, the model compares favourably to the model proposed by Ref. [73], which is valid for much higher Weber numbers.

As the numerical model has been shown to be highly accurate, it can be used to model various other droplet impact scenarios, ranging from micron size droplet impact, to impacts with higher $W e$, where the solidifying layer may touch the droplet interface before it has fully spread [73]. An extension of the regime diagram is of major interest to industry and specifically the rebounce behaviour of (solidifying) droplets deserves a careful approach. Furthermore, situations of interest for industry may be studied, like the depositions of droplets on droplets, or the deposition of droplets next to each other, in 3D. The model approach can be extended by including temperature dependent parameters, for instance surface tension, leading to droplet solidification where thermal Marangoni effects may play a role. 


\section{A. Parameter sensitivity}

\section{A Parameter sensitivity}

\section{A.1 Mesh dependence}

For the case without contact resistance, a mesh refinement study is performed. Since in GERRIS adaptive mesh refinement is used to reach high resolutions, only the relevant areas have highest resolution, i.e. the droplet-air interface and the solid-liquid interface. The liquid in the droplet itself had a resolution of 86 cells per droplet diameter in all cases. The high resolution zones range from 86 cells per droplet diameter to 688 cells per droplet diameter. The increment is performed in 4 steps with an increase of resolution of factor 2 in each increment.

(a)
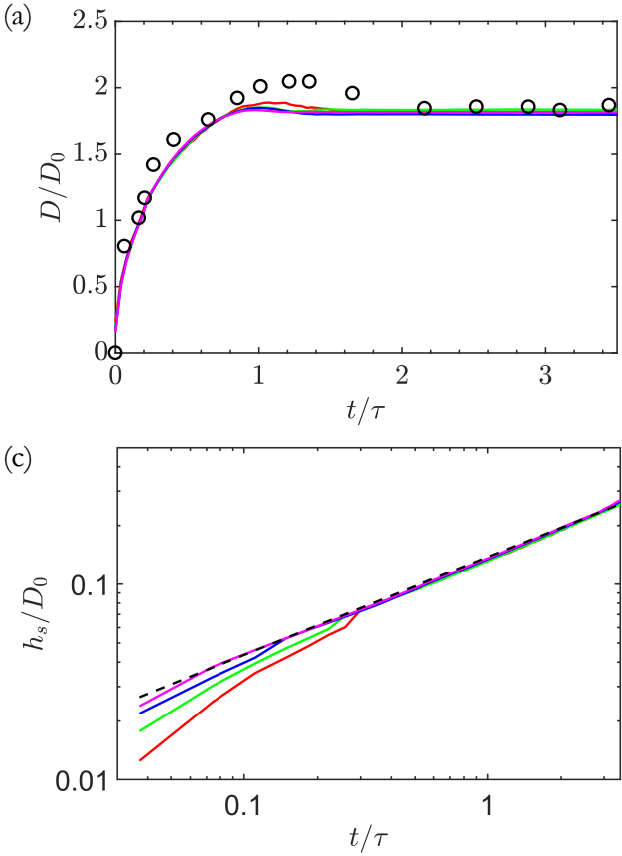

(b)

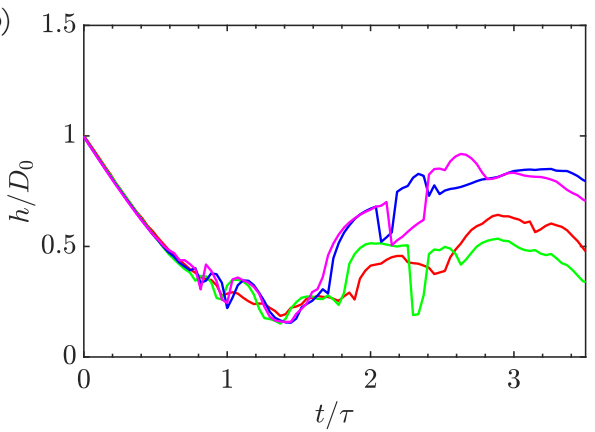

Figure 4.13: Comparison of (a) the spreading rate and (b) the droplet height for various resolutions. The black dots show the experimental results of Ref. [57]. (c) Comparison of the solidification height at the droplet centre to the Stefan problem (- - -). Number of cells per droplet diameter: — : 86, —: $172,-: 344,-: 688$.

The various resolutions show similar behaviour in spreading and retraction rate with regards to the diameter of spreading, see figure 4.13a. A higher resolution gives a slightly smaller maximum spreading diameter. This is due to the mushy sink term $C_{m u s h}$ in the momentum equation. Since it becomes dominant for high solidification fractions, for a larger cell size (or a lower heat conductivity), it takes longer to increase the value of this sink term and thus reduce momentum during solidification. The 

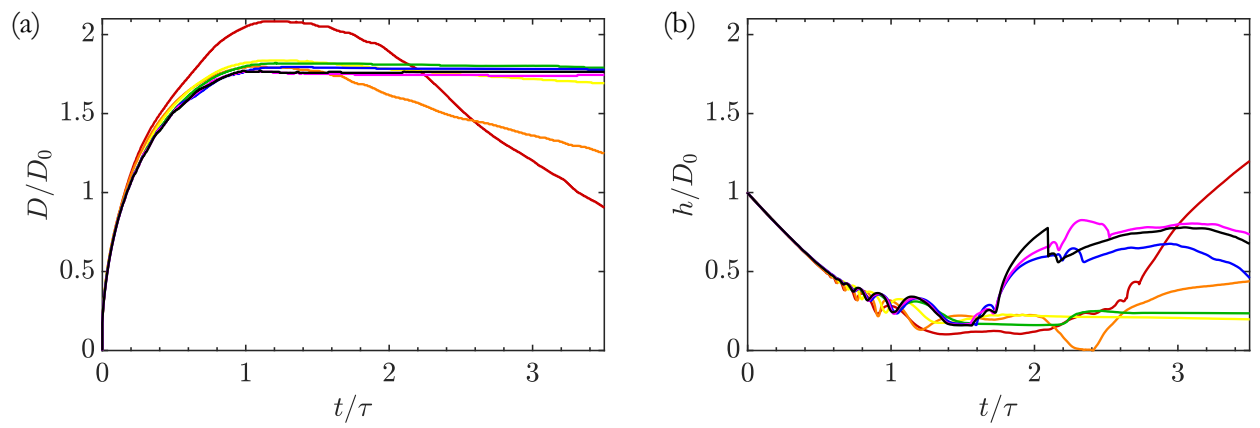

Figure 4.14: Comparison of the spreading rate (a) and droplet height (b) for various values of the sink term $C_{\text {mush }}$, with $\varepsilon=10^{-3} . C_{\text {mush }}=10^{3}(-), C_{\text {mush }}=10^{4}(-), C_{\text {mush }}=10^{5}$ $(-), C_{\text {mush }}=10^{6}(-), C_{\text {mush }}=10^{7}(-), C_{\text {mush }}=10^{8}(-), 10^{9}(-)$.

behaviour of the oscillation of the centre of the droplets does change with increasing resolution. The lower resolutions are not able to capture the full capillary behaviour of the droplet surface. The central height, figure $4.13 \mathrm{~b}$ is similar for the two highest resolutions. The slight differences are caused by the difference in solidification fraction per cell and the dampening effect on the droplet, but not significant in droplet spreading behaviour.

The solidification height at the centre of the droplet is compared to the Neumann solution to the 1-D Stefan problem in figure $4.13 \mathrm{c}$. In this system, the moving front of solidification is found for a semi-infinite liquid with a constant temperature boundary condition [77]. For all resolutions the numerical method converges to the analytical solution at the centre of the droplet. At the end of the process of droplet solidification, a small overshoot with respect to the analytical solution occurs. This is due to the heat transfer from air to the droplet, which slightly increases the cooling rate of the droplet. To have high enough resolution, all further calculations are performed using a resolution of 344 cells per droplet diameter.

\section{A.2 Influence of the source term}

Dendritic arm spacing depends on solidification speed, hence $C_{\text {mush }}$ ideally would be rate dependent. However, since the exact dendritic behaviour of solidification cannot be calculated within the current model, a typical arm spacing of $d_{d}=40 \mu \mathrm{m}$ is used, which lies in the range of results of earlier models and experimental results [61-63]. This leads to a typical value for $C_{m u s h}=1.7 \cdot 10^{8}$. In previous droplet solidification studies values typically in between $10^{3}$ and $10^{5}$ were used $[37,42,78,79]$. The study by Ref. [32] set $C_{\text {mush }}$ to the higher value of $10^{9}$. Here, a comparison of the effect of this damping factor is made for the impact case of a $2.7 \mathrm{~mm}$ tin droplet, without using 

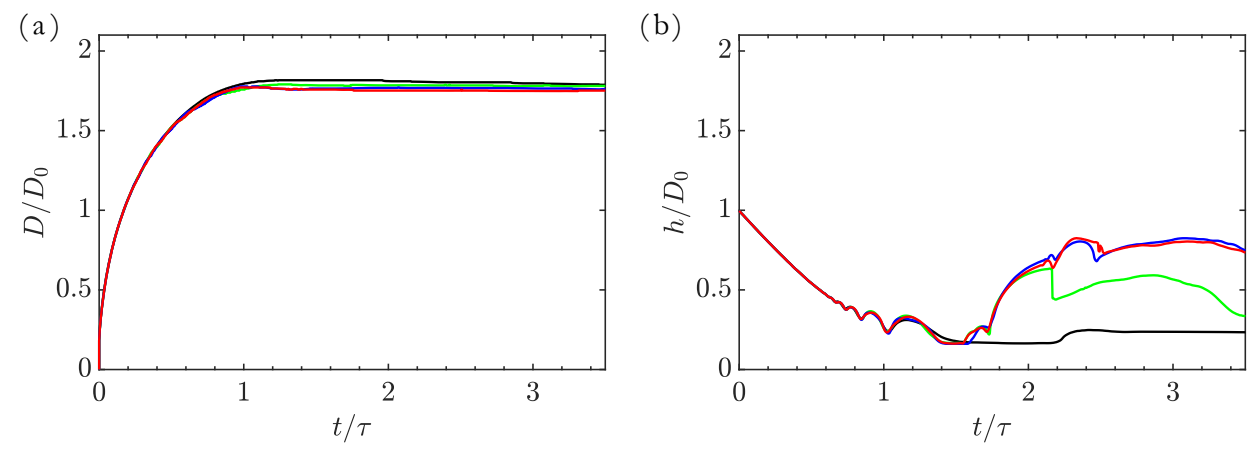

Figure 4.15: Comparison of the spreading rate (a) and droplet height (b) for various values of the term $\varepsilon$, with $C_{\text {mush }}=2 \cdot 10^{8} . \varepsilon=0.1(-) \varepsilon=0.01(\square) \varepsilon=10^{-3}(-) \varepsilon=10^{-4}(\square)$

any heat resistance.

Figure 4.14 shows the spreading behaviour for various value of $C_{m} u s h$. Before maximum spreading of the droplet, there is no large variation in spreading behaviour when changing the mushy zone constant $C_{\text {mush }}$. For $C_{\text {mush }}=10^{3}$ and $C_{\text {mush }}=10^{4}$, the damping term has no significant effect on droplet behaviour. The complete droplet retracts. For higher values of $C_{m u s h}$, the droplet spreading behaviour solidifies to a pinning diameter. The differences in spreading behaviour is minute. For the center height of the droplet, $C_{m u s h} \leq 10^{4}$ much higher re-bounce is observed, as compared to $C_{\text {mush }}=10^{5}$. For very high values, above $C_{m u s h}=10^{6}$, results of central droplet height converge to similar behaviour. The sensitivity of the method to changes in the source term value show that for early times the model functions well to predict droplet behaviour. However, quantitative behaviour at late times, e.g. re-bounce height, needs to be considered carefully.

The effect of $\varepsilon$ in equation (4.11), is similar to the behaviour of $C_{\text {mush }}$. Using a smaller value of $\varepsilon$ leads to a larger effect of the sink term. For the various values of $\varepsilon$ that are tested, no significant difference can be observed for the spreading of the droplet (figure 4.15). However, again, for the height of the droplet at the centre location, a larger deviation is observed. The behaviour for the oscillations of the centre of the droplet are similar for values of $\varepsilon$ of $10^{-3}$ and $10^{-4}$, when keeping the value of the damping term constant.

\section{A.3 Time step limit}

In cells where the liquid is almost solidified, $J \longrightarrow 1$, and the source term $S_{j}$ in equation (4.3) will be dominant. The time progression of the momentum equation then simplifies to:

$$
\frac{\partial \vec{u}}{\partial t}=-\frac{C_{m u s h}}{\rho \varepsilon} \vec{u},
$$


To show the stability restriction, a simple time explicit scheme is taken as an example. This leads to the discretised relation:

$$
\frac{u^{n+1}-u^{n}}{\Delta t}=-\frac{C_{m u s h}}{\rho \varepsilon} u^{n},
$$

The velocity ratio must be monotonically decreasing, to prevent oscillations of the solutions. This criterium can be used to rewrite equation (4.27) in terms of the velocity ratio:

$$
\left|\frac{u^{n+1}}{u^{n}}\right|=\left|1-\frac{C_{m u s h}}{\rho \varepsilon} \Delta t\right| \leq 1,
$$

limiting the time step to:

$$
\Delta t \leq 2 \frac{\rho \varepsilon}{C_{m u s h}} .
$$

Interestingly, using the porosity approach leads to a severe time step restriction, independent of cell size. 


\section{References}

[1] S. Chandra and P. Fauchais, "Formation of solid splats during thermal spray deposition", J. Therm. Spray Technol. 18, 148-180 (2009).

[2] M. Vaezi, H. Seitz, and S. Yang, "A review on 3D micro-additive manufacturing technologies”, Int. J. Adv. Manuf. Technol. 67, 1721-1754 (2013).

[3] D. J. Hayes, D. B. Wallace, and W. R. Cox, "MicroJet printing of solder and polymers for multi-chip modules and chip-scale packages", SPIE Proc. Ser. IMAPS'99, 242-247 (1999).

[4] Q. Liu and M. Orme, "High precision solder droplet printing technology and the state-of-the-art”, J. Mater. Process. Technol. 115, 271-283 (2001).

[5] J. Luo, L. H. Qi, S. Y. Zhong, J. M. Zhou, and H. J. Li, "Printing solder droplets for micro devices packages using pneumatic drop-on-demand (DOD) technique", J. Mater. Process. Technol. 212, 2066-2073 (2012).

[6] S. F. S. Shirazi, S. Gharehkhani, M. Mehrali, H. Yarmand, H. S. C. Metselaar, N. Adib Kadri, and N. A. A. Osman, "A review on powder-based additive manufacturing for tissue engineering: selective laser sintering and inkjet 3D printing.", Sci. Technol. Adv. Mater. 16, 033502 (2015).

[7] Y. Guo, H. Patanwala, B. Bognet, and A. W. Ma, "Inkjet and inkjet-based 3D printing: Connecting fluid properties and printing performance", Rapid Prototyp. J. 23, 562-576 (2017).

[8] F. Gao and A. A. Sonin, "Precise Deposition of Molten Microdrops: The Physics of Digital Microfabrication”, Proc. R. Soc. A Math. Phys. Eng. Sci. 444, 533-554 (2006).

[9] C. Visser, R. Pohl, C. Sun, G. Römer, B. Huis In 'T Veld, and D. Lohse, "Toward 3D Printing of Pure Metals by Laser-Induced Forward Transfer", Adv. Mater. 27, 4087-4092 (2015).

[10] M. Rein, "Phenomena of liquid drop impact on solid and liquid surfaces", Fluid Dyn. Res. 12, 61-93 (1993).

[11] A. Yarin, "Drop impact dynamics: Splashing, Spreading, Receding, Bouncing...”, Annu. Rev. Fluid Mech. 38, 159-192 (2006).

[12] C. Josserand and S. T. Thoroddsen, "Drop Impact on a Solid Surface", Annu. Rev. Fluid Mech. 48, 365-391 (2016). 
[13] D. Khojasteh, M. Kazerooni, S. Salarian, and R. Kamali, "Droplet impact on superhydrophobic surfaces: A review of recent developments", J. Ind. Eng. Chem. 42, 1-14 (2016).

[14] A. Yarin, I. V. Roisman, and C. Tropea, Collision phenomena in liquids and solids, 1 edition (Cambride University Press, Cambride) (2017).

[15] S. Popinet, "Numerical Models of Surface Tension", Annu. Rev. Fluid Mech. 50, 49-75 (2017).

[16] S. Popinet, "An accurate adaptive solver for surface-tension-driven interfacial flows”, J. Comput. Phys. 228, 5838-5866 (2009).

[17] A. Outzourhit and J. U. Trefny, "Simple apparatus for thermal conductivity measurements of unconsolidated powders", Exp. Heat Transf. 7, 319-331 (1994).

[18] T. Bennett and D. Poulikakos, "Splat-quench solidification: estimating the maximum spreading of a droplet impacting a solid surface”, J. Mater. Sci. 28, 963-970 (1993).

[19] R. H. Rangel and X. Bian, "Metal-droplet deposition model including liquid deformation and substrate remelting", Int. J. Heat Mass Transf. 40, 2549-2564 (1997).

[20] J. P. Delplanque and R. H. Rangel, "An improved model for droplet solidification on a flat surface”, J. Mater. Sci. 32, 1519-1530 (1997).

[21] H. Liu, E. J. Lavernia, and R. H. Rangel, "Numerical simulation of substrate impact and freezing of droplets in plasma spray processes", J. Phys. D. Appl. Phys. 26, 1900-1908 (1993).

[22] SanMarchi;, H. Liu, E. Lavernia, R. H. Rangel, A. Sickinger, and E. Muehlberger, "Numerical analysis of the deformation and solidification of a single droplet impinging onto a flat substrate", J. Mater. Sci. 28, 3313-3321 (1993).

[23] M. Pasandideh-Fard, R. Bhola, S. Chandra, and J. Mostaghimi, "Deposition of tin droplets on a steel plate : Simulations and experiments", Int. J. Heat Mass Transf. 41, 2929-2945 (1998).

[24] M. Pasandideh-Fard, M. Bussmann, and S. Chandra, "Simulating Droplet Impact on a Substrate of Arbitrary Shape", At. Sprays 11, 397-414 (2014).

[25] H. Zhang, X. Y. Wang, L. L. Zheng, and X. Y. Jiang, "Studies of splat morphology and rapid solidification during thermal spraying", Int. J. Heat Mass Transf. 44, 4579-4592 (2001). 
References

[26] M. Pasandideh-Fard, S. Chandra, and J. Mostaghimi, "A three-dimensional model of droplet impact and solidification", Int. J. Heat Mass Transf. 45, 22292242 (2002).

[27] M. Pasandideh-Fard, V. Pershin, S. Chandra, and J. Mostaghimi, "Splat shapes in a thermal spray coating process: Simulations and experiments", J. Therm. Spray Technol. 11, 206-217 (2002).

[28] A. N. Cherepanov, V. N. Popov, and O. P. Solonenko, "Crystallization dynamics of metallic droplet modified with nanoinoculators at its impact with substrate", Thermophys. Aeromechanics 17, 383-390 (2010).

[29] S. Alavi, M. Passandideh-Fard, and J. Mostaghimi, "Simulation of semi-molten particle impacts including heat transfer and phase change", J. Therm. Spray Technol. 21, 1278-1293 (2012).

[30] S. Vincent, C. L. Bot, F. Sarret, E. Meillot, J. P. Caltagirone, and L. Bianchi, "Penalty and Eulerian-Lagrangian VOF methods for impact and solidification of metal droplets plasma spray process", Comput. Fluids 113, 32-41 (2015).

[31] C. Le Bot, S. Vincent, and E. Arquis, "Impact and solidification of indium droplets on a cold substrate", Int. J. Therm. Sci. 44, 219-233 (2005).

[32] S. Alavi and M. Passandideh-Fard, "Numerical simulation of droplet impact and solidification including thermal shrinkage in a thermal spray process", Front. Heat Mass Transf. 2, 023007 (2011).

[33] Y. Z. Zheng, Q. Li, Z. H. Zheng, J. F. Zhu, and P. L. Cao, "Modeling the impact, flattening and solidification of a molten droplet on a solid substrate during plasma spraying”, Appl. Surf. Sci. 317, 526-533 (2014).

[34] V. Ramanuj and A. Tong, "A numerical model for a hypoeutectic alloy droplet deposition with non-equilibrium solidification”, J. Mater. Sci. 52, 6034-6049 (2017).

[35] M. Pasandideh-Fard and J. Mostaghimi, "On the spreading and solidification of molten particles in a plasma spray process effect of thermal contact resistance", Plasma Chem. Plasma Process. 16, S83-S98 (1995).

[36] M. Chung and R. H. Rangel, "Parametric study of metal droplet deposition and solidification process including contact resistance and undercooling effects", Int. J. Heat Mass Transf. 44, 605-618 (2001).

[37] W. Wang, F. J. Hong, H. H. Qiu, and P. Cheng, "The impact of thermal contact conductance on the spreading and solidification of a droplet on a substrate", Heat Transf. Eng. 27, 68-80 (2006). 
[38] S. Popinet, "Gerris: A tree-based adaptive solver for the incompressible Euler equations in complex geometries", J. Comput. Phys. 190, 572-600 (2003).

[39] F. Civan, Porous media transport phenomena (John Wiley \& Sons) (2011).

[40] I. Farup and A. Mo, "Two-phase modeling of Mushy zone parameters associated with hot tearing”, Metall. Mater. Trans. A Phys. Metall. Mater. Sci. 31, 14611472 (2000).

[41] A. Faghri and T. L. Bergman, "Three-Dimensional Sintering of Two-Component Metal Powders", J. Heat Transfer 122, 150-158 (2000).

[42] H. Tabbara and S. Gu, "Numerical study of semi-molten droplet impingement", Appl. Phys. A Mater. Sci. Process. 104, 1011-1019 (2011).

[43] M. Kaviany, Principles of Heat Transfer in Porous Media (Springer Science \& Business Media) (1991).

[44] C. Beckermann and R. Viskanta, "Double-diffusive convection during dendritic solidification of a binary mixture", Phys. Chem. Hydrodyn. 10, 195-213 (1988).

[45] H. Inoue, S. Asai, and I. Muchi, "Theoretical Analysis and Model Experiments on Formation Mechanism of V-type Segregation”, Tetsu-to-Hagane 71, 11321139 (2017).

[46] S. Patankar, Numerical heat transfer and fluid flow (CRC press) (1980).

[47] D. B. van Dam and C. Le Clerc, "Experimental study of the impact of an ink-jet printed droplet on a solid substrate", Physics of Fluids 16, 3403-3414 (2004).

[48] S. Thoroddsen, T. Etoh, K. Takehara, N. Ootsuka, and Y. Hatsuki, "The air bubble entrapped under a drop impacting on a solid surface", Journal of Fluid Mechanics 545, 203-212 (2005).

[49] S. Mandre, M. Mani, and M. P. Brenner, "Precursors to splashing of liquid droplets on a solid surface", Physical review letters 102, 134502 (2009).

[50] J. M. Kolinski, S. M. Rubinstein, S. Mandre, M. P. Brenner, D. A. Weitz, and L. Mahadevan, "Skating on a film of air: drops impacting on a surface", Physical review letters 108, 074503 (2012).

[51] S. Mandre and M. P. Brenner, "The mechanism of a splash on a dry solid surface", Journal of Fluid Mechanics 690, 148-172 (2012).

[52] W. Bouwhuis, R. C. A. Van Der Veen, T. Tran, D. L. Keij, K. G. Winkels, I. R. Peters, D. Van Der Meer, C. Sun, J. H. Snoeijer, and D. Lohse, "Maximal air bubble entrainment at liquid-drop impact", Phys. Rev. Lett. 109, 264501 (2012). 
[53] C. Visser, P. Frommhold, S. Wildeman, R. Mettin, D. Lohse, and C. Sun, "Dynamics of high-speed micro-drop impact: Numerical simulations and experiments at frame-to-frame times below 100 ns", Soft Matter 11, 1708-1722 (2015).

[54] M. J. Thoraval, K. Takehara, T. G. Etoh, and S. T. Thoroddsen, "Drop impact entrapment of bubble rings", J. Fluid Mech. 724, 234-258 (2013).

[55] S. Afkhami, S. Zaleski, and M. Bussmann, "A mesh-dependent model for applying dynamic contact angles to VOF simulations", J. Comput. Phys. 228, 5370-5389 (2009).

[56] J. R. Simonson, Engineering Heat Transfer, 2 edition (CRC Press LLC, Boca Raton, FL 33431) (2015).

[57] S. D. Aziz and S. Chandra, "Impact, recoil and splashing of molten metal droplets", Int. J. Heat Mass Transf. 43, 2841-2857 (2000).

[58] Y. Heichal and S. Chandra, "Predicting Thermal Contact Resistance Between Molten Metal Droplets and a Solid Surface", J. Heat Transfer 127, 1269-1275 (2005).

[59] D. W. Hahn and M. N. Özisik, Heat conduction (John Wiley \& Sons) (2012).

[60] J. I. Lienhard and J. H. V. Lienhard, A heat transfer textbook, 3, 3 edition (Phlogiston Press, Cambridge, Massachusetts) (2008).

[61] T. Okamoto and K. Kishitake, "Dendritic structure in unidirectionally solidified aluminum, tin, and zinc base binary alloys", J. Cryst. Growth 29, 137-146 (1975).

[62] D. Bouchard and J. S. Kirkaldy, "Prediction of dendrite arm spacings in unsteadyand steady-state heat flow of unidirectionally solidified binary alloys", Metall. Mater. Trans. B Process Metall. Mater. Process. Sci. 28, 651-663 (1997).

[63] F. Ochoa, J. J. Williams, and N. Chawla, "The effects of cooling rate on microstructure and mechanical behavior of Sn-3.5Ag solder", Jom 55, 56-60 (2003).

[64] Y. Renardy, S. Popinet, L. Duchemin, M. Renardy, S. Zaleski, C. Josserand, M. A. Drumright-Clarke, D. Richard, C. Clanet, and D. Quéré, "Pyramidal and toroidal water drops after impact on a solid surface", J. Fluid Mech. 484, 69-83 (2003). 
[65] H. Ding, E. Q. Li, F. H. Zhang, Y. Sui, P. D. M. Spelt, and S. T. Thoroddsen, "Propagation of capillary waves and ejection of small droplets in rapid droplet spreading”, J. Fluid Mech. 697, 92-114 (2012).

[66] Y. Hung, M. Wang, J. Huang, and S. Lin, "A study on the impact velocity and drop size for the occurrence of entrapped air bubbles - Water on parafilm", Exp. Therm. Fluid Sci. 48, 102-109 (2013).

[67] H. Hu, L. Chen, S. Huang, and B. Song, "Rebound behaviors of droplets impacting on a superhydrophobic surface", Sci. China Physics, Mech. Astron. 56, 960-965 (2013).

[68] D. Richard, C. Clanet, and D. Quéré, "Surface phenomena: Contact time of a bouncing drop", Nature 417, 811-812 (2002).

[69] T. Mao, D. Kuhn, and H. Tran, "Spread and Rebound of Liquid Droplets upon Impact on Flat Surfaces", AIChE J. 43, 2169-2179 (1997).

[70] A. Mongruel, V. Daru, F. Feuillebois, and S. Tabakova, "Early post-impact time dynamics of viscous drops onto a solid dry surface", Phys. Fluids 21, 032101 (2009).

[71] N. Laan, K. De Bruin, D. Bartolo, C. Josserand, and D. Bonn, "Maximum diameter of impacting liquid droplets", Phys. Rev. Appl. 2, 044018 (2014).

[72] S. Wildeman, C. Visser, C. Sun, and D. Lohse, "On the spreading of impacting drops”, J. Fluid Mech. 805, 636-655 (2016).

[73] M. V. Gielen, R. de Ruiter, R. B. Koldeweij, D. Lohse, J. H. Snoeijer, and H. Gelderblom, "Solidification of liquid metal drops during impact", Journal of Fluid Mechanics 883 (2020).

[74] D. Richard and D. Quéré, "Bouncing water drops", Europhys. Lett. 50, 769-775 (2000).

[75] K. Okumura, F. Chevy, D. Richard, D. Quéré, and C. Clanet, "Water spring: A model for bouncing drops", Europhys. Lett. 62, 237-243 (2003).

[76] A. Bejan and A. D. Kraus, Heat Transfer Handbook, volume 67 (John Wiley \& Sons) (2003).

[77] H. S. Carslaw and J. C. Jaeger, Conduction of heat in solids, 2 edition (Oxford Science Publications, Oxford, UK) (1959). 
References

[78] V. R. Voller and C. Prakash, "A fixed grid numerical modelling methodology for convection-diffusion mushy region phase-change problems", Int. J. Heat Mass Transf. 30, 1709-1719 (1987).

[79] S. Kamnis and S. Gu, "Numerical modelling of droplet impingement", J. Phys. D. Appl. Phys. 38, 3664-3673 (2005). 



\section{5 Marangoni-driven spreading of miscible liquids in the binary pendant drop geometry}

When two liquids with different surface tensions come into contact, the liquid with lower surface tension spreads over the other liquid. This Marangoni-driven spreading has been studied for various geometries and surfactants, but the dynamics of miscible liquids in the binary geometry (drop-drop) has hardly been investigated. In this chapter we use stroboscopic illumination by nanosecond laser pulses to temporally resolve the distance $L(t)$ over which a low-surface-tension drop spreads over a miscible high-surface-tension drop. $L(t)$ is measured as a function of time, $t$, for various surface tension differences between the liquids and for various viscosities, revealing a power-law $L(t) \sim t^{\alpha}$ with a spreading exponent $\alpha \approx 0.75$. This value is consistent with previous results for viscosity-limited spreading over a deep bath. The universal power law $\tilde{L} \propto \tilde{t}^{3 / 4}$ that describes the dimensionless distance $\tilde{L}$ as a function of the dimensionless time $\tilde{t}$ reasonably captures our experiments, as well as previous experiments for different geometries, miscibilities, and surface tension modifiers (solvents and surfactants). The range of this power law remarkably covers ten orders of magnitude in dimensionless time. This result enables engineering of drop encapsulation for various liquid-liquid systems.

*Published as: "Marangoni-driven spreading of miscible liquids in the binary pendant drop geometry", R.B.J. Koldeweij, B.F. van Cappeleveen, D. Lohse and C.W. Visser, Soft Matter 15, 8525-8531 (2019) 


\subsection{Introduction}

(a)

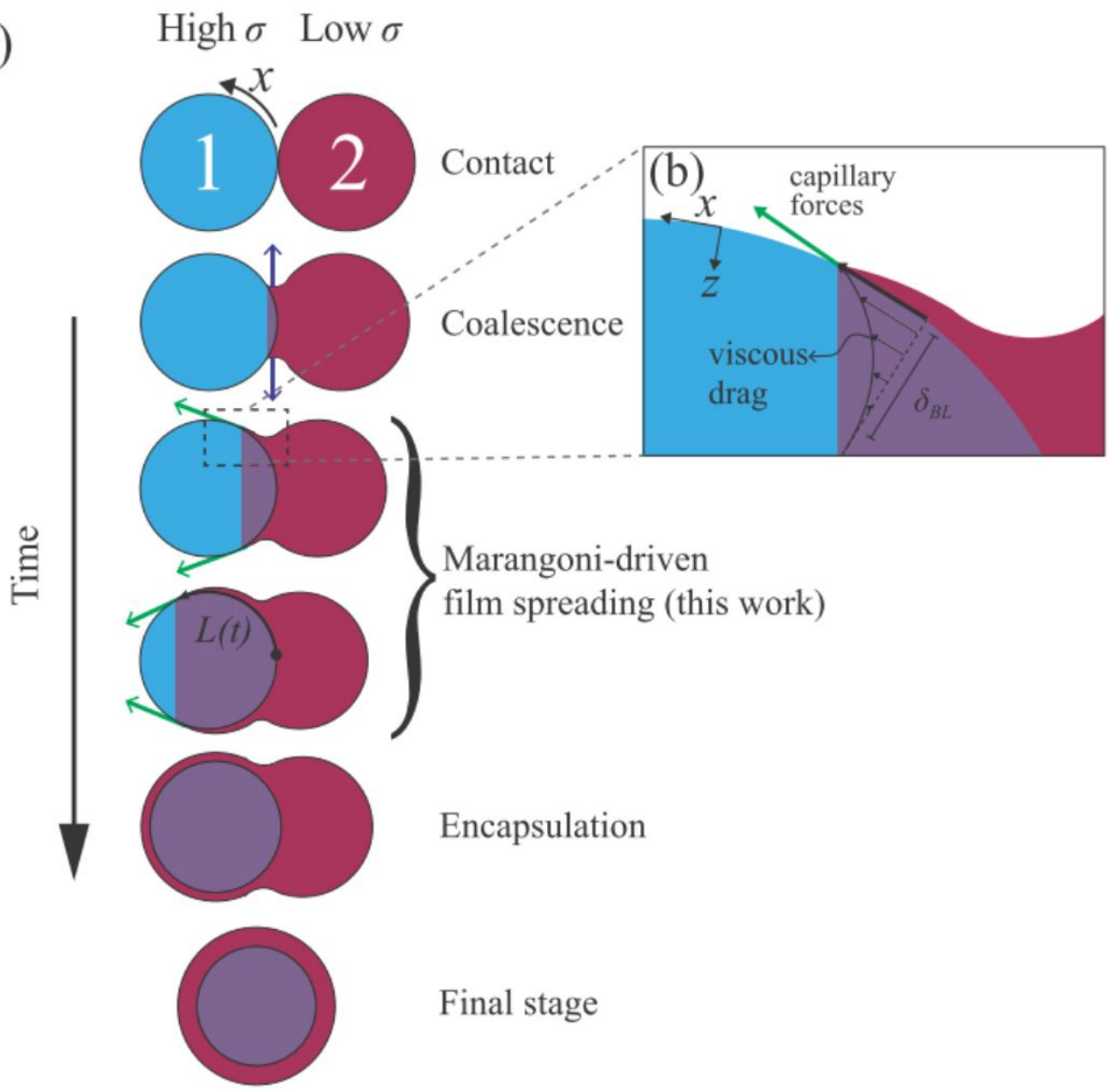

Figure 5.1: Overview of binary drop spreading; $(a)$ At $t=0$ the drops touch. Initially, coalescence radially expands the neck due to local curvature as indicated by the blue arrows [1]. This regime is followed by Marangoni-driven spreading of the drop with lower surface tension over the other one (green arrows). Ultimately, this mechanism results in encapsulation of drop 1. (b) Indicative flows of Marangoni-driven spreading, where the Marangoni stress is balanced by a viscous boundary layer.

Liquids of low surface tension spread over liquid with high surface tension, which is known as Marangoni spreading. This phenomenon has been studied in various contexts, such as oil spills on the sea [2-6], pulmonary surfactant replacement therapy [7, 8], foam destruction [9], and fabrication of soft polymer actuators [10]. Recently, Marangoni spreading has been used for encapsulation of a high-surface tension drop by a lower surface tension liquid, as shown in figure 5.1a. This mechanism is used in pharmacy [11, 12], for manufacturing of biomaterials [13], electronics [14], food 


\subsection{Introduction}

and vitamins [15], microparticles with multiple compartments [16, 17], and rapid 3D-bioprinting with in-air microfluidics [18].

The morphological outcome of two colliding drops in air, such as encapsulation or breakup, has been assessed for miscible [11, 18] and immiscible [19-23] liquid pairs with different surface tensions. Encapsulation can also be achieved by impacting drops with different sizes [24] or different viscosities [25]. However, to our best knowledge, their surface-tension-driven encapsulation dynamics have hardly been visualized. Drops can also be encapsulated by gentle deposition onto a bath with a lower surface tension, but here the film dynamics were only assessed during coalescence (an earlier regime that precedes encapsulation) [26] or for surface tension induced necking $[27,28]$, rather than encapsulation. Encapsulation in the binary drop geometry was studied for submerged drop pairs, revealing a constant velocity of the spreading film both experimentally [29, 30] and numerically [24, 31]. However, it is unclear whether this result also applies to drop pairs in air, since the viscosity of the surrounding liquid plays an important role.

Knowledge of Marangoni spreading over a flat liquid surface with a higher surface tension could also provide clues to describe spreading over drops. This topic has been studied in many configurations, of which most can be classified according to four criteria: (i) liquid-driven versus surfactant-driven spreading, (ii) miscible versus immiscible liquid pairs, (iii) shallow versus deep liquid "carrier" layers, and (iv) spreading from a finite reservoir versus a source. Here, we focus on spreading of ethanol/water mixtures over water drops, corresponding to surfactant-free and miscible liquid pairs. For a drop pair, the transition between deep and shallow carrier layers may depend on the thickness of the flow-induced viscous boundary layer as sketched in figure 5.1b. Deep-layer behavior is expected if the boundary layer thickness $\delta_{B L}<D_{1} / 4$, with $D_{1} \approx 2 \mathrm{~mm}$ the inner drop's diameter [32]. Using typical values for the density $\rho=1000 \mathrm{~kg} / \mathrm{m}^{3}$, viscosity $\eta=1 \mathrm{mPa} \mathrm{s}$, and time $t=10 \mathrm{~ms}$, we obtain $\delta_{B L}=\left(\eta_{1} t / \rho_{1}\right)^{1 / 2} \approx 0.1 \mathrm{~mm}$. Therefore, the comparison to the spreading of a drop over a deep layer is considered. Finally, the outer drop is assumed to be an infinite source, as its volume suffices to form a thick film around the inner drop. The configuration of a spreading drop over a deep layer was first studied by Suciu et al. [33-36] for a quasi-steady regime. However, the preceding initial expansion of the film is the relevant regime for drop encapsulation.

The spreading distance $L(t)$ of a low-surface tension liquid over a liquid with a higher surface tension can be described by a power law [4]:

$$
L(t)=\beta t^{\alpha}
$$

The spreading exponent $\alpha$ and the dimensional prefactor $\beta$, are usually reported as a function of the geometric and material parameters [37], and are the scope of this study. The canonical result for spreading on a deep bath is $\alpha=3 / 4$ and $\beta=k S^{1 / 2}(\rho \eta)^{-1 / 4}$, 
in which $k$ is a dimensionless constant. $S \approx \Delta \sigma$ represents the spreading parameter for liquid pairs in air, and $\Delta \sigma=\sigma_{1}-\sigma_{2}$ is the surface tension difference between the liquids. These values follow from balancing the surface tension gradient with dissipation in the viscous boundary layer that develops while spreading on a deep layer [5, 6, 38-40]. Experiments performed for immiscible, non-evaporative liquids [41, 42], immiscible surfactant solutions [43], liquid spreading over a liquid covered with insoluble surfactants [44], and for immiscible micro-drops spreading over free-flowing thin films [32] validated this scaling argument. For miscible surfactant solutions, the spreading exponent is maintained around $\alpha=0.75$ for low solubility [45, 46], but it can decrease to $\alpha=0.4$ for highly soluble surfactants [47]. An indicative value for the constant $k \approx 0.88$ applies to spreading of immiscible liquids in the radial geometry, but values in the range of $k=0.665$ to $k=1.52$ have been reported [41].

For spreading of a low $-\sigma$ drop over a deep bath of a miscible liquid as considered here, a spreading exponent in the range $\alpha=0.53 \pm 0.03$ was measured for nitroethane, ethyl acetate [48], and isopropanol drops [49] deposited on water. Molecular dynamics simulations of ethanol solutions spreading over water revealed a similar exponent of $\alpha=0.55 \pm 0.05$ [50]. These reduced values, as well as a decrease of the prefactor to $k \approx 0.3$, were attributed to dissolution of the spreading liquid into the bath by convective rolls that form at the film's edge $[48,51]$. A similar reduction in the spreading exponent is observed for evaporation-driven formation of convective rolls [41]. An even lower exponent $(\alpha \approx 0.25)$ was measured for ethanol drops on a water bath [52], and explained by balancing $\Delta \sigma$ with viscous dissipation within the spreading film [53].

As the existing literature indicates that spreading exponents $1 / 4 \lesssim \alpha \lesssim 1$ could apply to the binary drop geometry, here we observe and quantify the Marangoni-driven spreading dynamics of miscible drop pairs. By encapsulating a fluorescent inner drop by an optically absorbing low- $\sigma$ liquid, we obtain the spreading distance as a function of time, the surface tension difference, and the viscosity. Subsequently, we determine the spreading exponents and compare these to systems with different geometries, surfactants and miscibilities. This chapter is organized as follows: In section 5.2, the experimental setup and liquids are described. The results and discussion are described in section 5.3, followed by the conclusions in section 5.4.

\subsection{Experimental set-up and materials}

To create the binary drop geometry, two pendant drops were dispensed from teflon needles (Hamilton Company). The needles were fed by identical syringes mounted on a syringe pump (Harvard PHD 2000), pumping with a typical rate of $0.25 \mathrm{~mL} \mathrm{~min}^{-1}$ that resulted in the formation of approximately 25 drops per minute. The needles were placed at a $2.5 \mathrm{~mm}$ center-to-center distance, resulting in drops with a diameter 

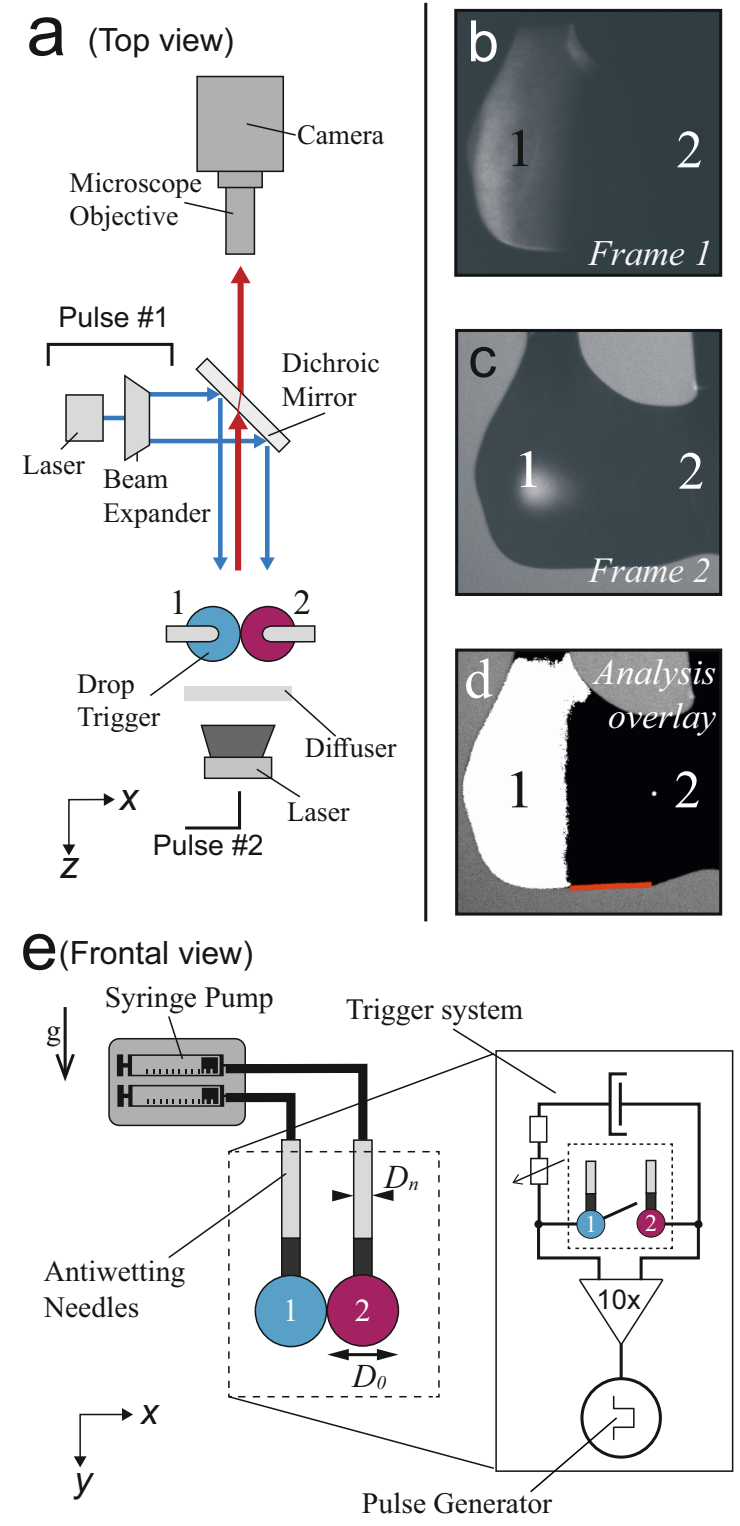

Figure 5.2: (a) Top view of the set-up. Drops 1 and 2 are illuminated by pulse \#1, as shown by the blue arrows. Fluorescent light is emitted only by drop 1 and passes the dichroic mirror, as indicated by the red arrow. (b) Example fluorescent image. (c) At virtually the same moment, pulse \#2 illuminates both drops from the back. The resulting bright-field image is shown. $(d)$ Processed overlay of both images. (e) Setup from the side-view perspective of the camera. (Inset) A pulse is generated when the drops closes an electric circuit. The delay time between this pulse and the image capture was controlled with a pulse generator. 


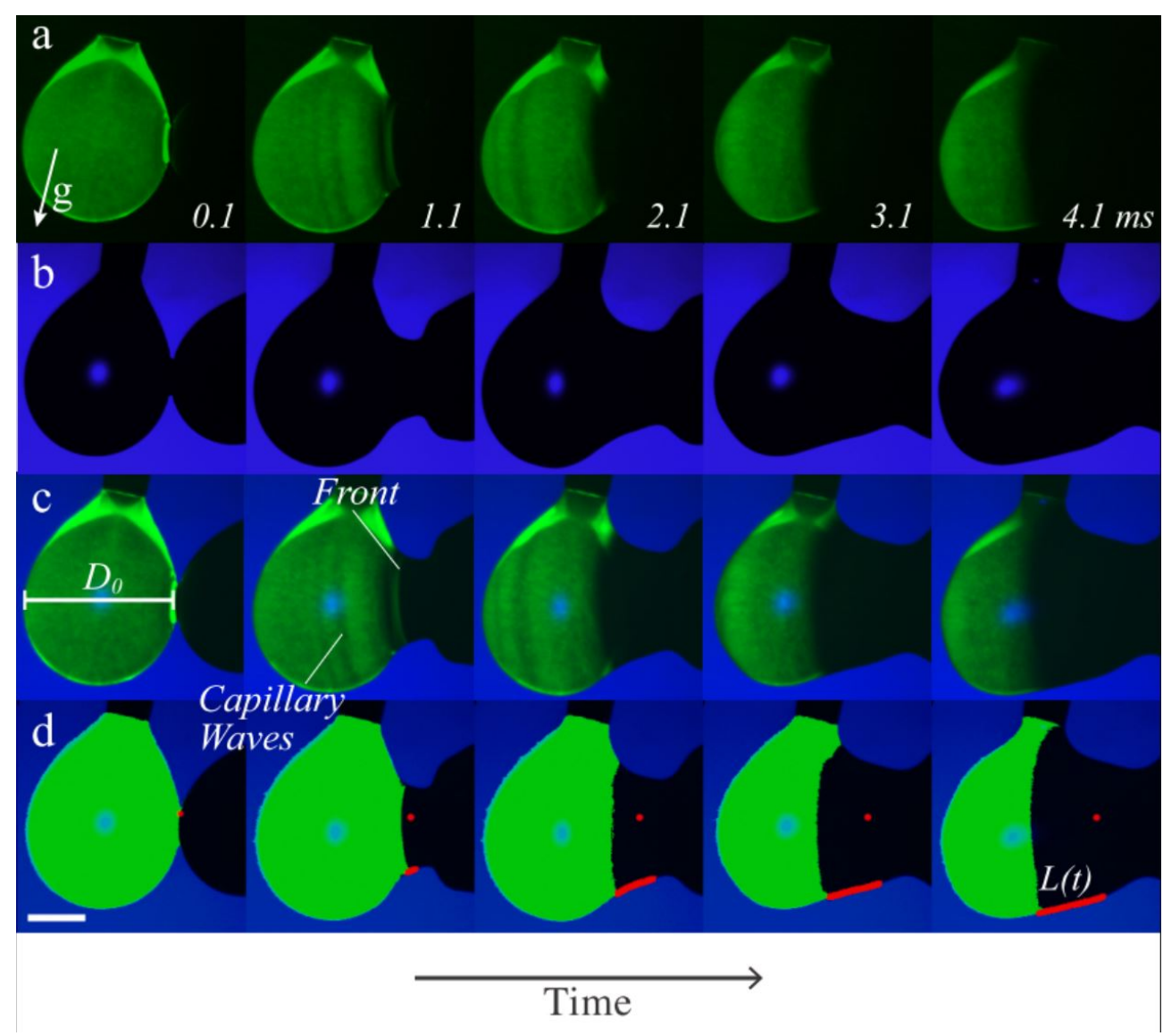

Figure 5.3: Image analysis procedure. (a) Typical darkfield image sequence, only showing fluorescent drop 1. The numbers indicate the time after first contact in ms. (b) Brightfield image sequence, showing the contours of both drops. (c) Overlay of (a) and (b), revealing the spreading film. Capillary waves and spreading front are observed as indicated. $(d)$ Result of the image analysis procedure. The red dot indicates the $x$-position of merging, while the red line indicates the spreading distance of the low-surface tension solution. The scale-bar indicates $1 \mathrm{~mm}$.

$D_{0}=2.5 \pm 0.5 \mathrm{~mm}$, just before the first contact. For each drop pair, the ratio was $0.95<D_{1} / D_{2}<1.05$. The high- $\sigma$ drop consisted of milli-Q water as a base, to which fluorescein (emission at $525 \mathrm{~nm}$ ) was added for fluorescent visualization. The low- $\sigma$ drop consists of a 15 vol\% inkjet printer ink solution (Brother LC-800), to provide an optically absorbing film that blocks the fluorescent light of the high- $\sigma$ drop during spreading. The surface tension gradient was modified by adding ethanol to the low- $\sigma$ drop, and measured by the hanging drop method as shown in supplementary figures 1 and 2. The measured values were constant in time (supplementary figure 3 ), confirming that evaporation does not affect the surface tension over the duration of 


\subsection{Experimental set-up and materials}

the drop formation in the experiments. The viscosity of the liquids was controlled by adding glycerol, as shown in supplementary figures 4 and 5 . The viscosity of glycerol-free mixtures of ethanol, water, and ink was measured to be $1.5 \pm 0.5 \mathrm{mPas}$.

The visualization setup is depicted in figure 5.2a. Stroboscopic imaging was used to generate two images of the drop pair at a controlled time after contact. The first image was illuminated with a pulsed laser (Litron Nano S PIV $400 \mathrm{~mJ}$, wavelength $532 \mathrm{~nm}$, pulse duration 8ns), of which the optical path is shown by the blue arrows in figure 5.2a. Only the fluorescent light is observed, as shown in figure 5.2b. The second frame was exposed by diffuse illumination from behind both drops, resulting in images of the drop contours as shown in figure 5.2c. Here, the pulse was provided by a second pulsed laser (Evergreen $600 \mathrm{~mJ}$, wavelength $532 \mathrm{~nm}$ ) that was diffused with a fluorescent diffuser (LaVision) to prevent fringes. The delay time between both laser pulses was set to $500 \mathrm{~ns}$, and the corresponding images were captured in separate frames of a dedicated dual-frame camera (PCO Sensicam qe). As this delay time is approximately 4 orders of magnitude shorter than the capillary time scale $\left(T_{\text {cap }}=\sqrt{\rho R_{0}^{3} / \sigma} \approx 5 \mathrm{~ms}\right)$, no significant motion occurs between frames 1 and 2. The frames were overlaid with excellent spatial collapse, as shown in figure $5.2 \mathrm{~d}$, revealing the spreading extent of the film and the outer contour of the drops. Time series were generated by repeating the above procedure for different delays between the moment of drop-drop contact $(t=0)$ and image capture. The moment of contact was obtained by closing an electrical circuit with the drops, as shown in Figure 5.2e (inset). The drops were made conductive by dissolving $1 \mathrm{vol} \% \mathrm{NaCl}$ into both liquids, which hardly affects the surface tension [54]. Experimental differences between two drop pairs sometimes result in 'flickering' of the video and additional noise on the measurements, as for example observed in figure 5.4 for the $20 \mathrm{mPas}$ and $50 \mathrm{mPas}$ drops. Still we use this stroboscopic method, as it enables visualizing both liquids at frame rates $>10^{4} \mathrm{~s}^{-1}$.

Figures 5.3a and 5.3b show an example time series of the fluorescent drop and both drops' contours, respectively. The overlay in figure $5.3 \mathrm{c}$ reveals the spreading extent of the film. The relative light transmission of $I / I_{0}=5 \%$ as compared to the uncovered (green) drop was chosen to determine the covered part with automated image analysis, corresponding to a film thickness of $63 \mu \mathrm{m}$. The exact value of this threshold had a minor influence on the spreading distance (see supplementary figures 6 and 7). Still, we would like to stress that we measure the spreading of relatively thick films that are relevant to encapsulation, rather than micrometer- or nanometer-thin films as reported previously $[36,55]$. The location of contact was determined in the first image after contact was measured. We traced the spreading along the bottom of the drop pair to prevent errors due to out-of-plane motion, as indicated by the red lines in figures $5.2 \mathrm{~d}$ and $5.3 \mathrm{~d}$. To reduce the risk of errors, we averaged three measurements of the spreading distance for each configuration and each time step, and performed 
scans of the control parameters $\Delta \sigma$ and $\eta$ over the largest feasible range for which spreading still occurs. Still, differences between individual drop pairs sometimes resulted in scatter of the data, as for example observed in figure 4 a for $\eta=10 \mathrm{mPas}$. To obtain the spreading rate for each measurement series, we used a nonlinear least squares power law fit on the measured spreading curves.

\subsection{Results and discussion}

The position of the spreading front $L(t)$ was measured as a function of time and viscosity, as shown in figure 5.4, revealing power-law behavior with an approximate scaling exponent $\alpha=3 / 4$ for low viscosities $\left(\eta_{1} \approx \eta_{2} \leq 20 \mathrm{mPas}\right)$. The prefactor is reasonably described by $k \approx 0.6$, i.e. $L(t)=0.6 \Delta \sigma^{1 / 2}(\rho \eta)^{-1 / 4} t^{3 / 4}$. Increasing the viscosity to $\eta_{1} \approx \eta_{2}=50 \mathrm{mPas}$ leads to a significant decrease in the spreading rate. The Ohnesorge number for this case is $O h=\eta / \sqrt{\rho \Delta \sigma D} \approx 0.2$, i.e. spreading seems to be significantly slower than predicted by equation 5.1 when global viscous forces become comparable to surface tension forces.

Figure 5.5a shows the spreading distance as a function of the surface tension difference, which was varied from $\Delta \sigma=0.4 \mathrm{mNm}^{-1}$ to $\Delta \sigma=23.2 \mathrm{mNm}^{-1}$. The spreading exponents are still consistent with $\alpha=3 / 4$, as shown in figure 5.5b. The prefactors exhibit substantial statistical errors and data scattering, but a value of $0.6 \Delta \sigma^{1 / 2}(\rho \eta)^{1 / 4}$ still reasonably well captures the measured data as shown in figure 5.5c. Spreading is inhibited for $\Delta \sigma=0.4 \mathrm{mN} \mathrm{m}^{-1}$, for which $O h \approx 0.15$.

The effect of changing the viscosity ratio $\eta_{1} / \eta_{2}$ between the drops is shown in figure 5.6a. The fastest spreading is observed for a ratio of unity $\left(\eta_{1}=\eta_{2} \approx 1.5 \mathrm{mPas}\right.$ ), as the viscosity of both liquids is set to their lowest values for this case. Increasing the viscosity of liquid 1 results in a decrease in spreading over the entire temporal domain. The spreading exponent $\alpha$ (figure 5.6b) does not depend on the viscosity ratio $\eta_{1} / \eta_{2}$ and is around $\alpha \approx 3 / 4$ for all cases. The reduction in spreading is captured by the prefactor, which is consistent with theory for viscosity ratios $\eta_{1} / \eta_{2} \geq 1$ (figure 5.6c). Measurements for which the viscosity of drop 2 is increased (that is, $\eta_{1} / \eta_{2}<1$ ), also exhibit reduced spreading. To assess whether this reduction reflects a transition to film-limited dissipation, we compare the dissipation in the spreading film and the boundary layer of drop 1 . The dissipation in the spreading film is described by $\Omega_{f} \eta_{2}(\dot{L} / L)^{2}$, with $\Omega_{f} \sim L h_{f}$ the film volume (assuming a 2-dimensional system) and $h_{f}$ the film thickness [53]. The dissipation in the boundary layer developing in drop 1 is described by $\Omega_{B L} \eta_{1}\left(\dot{L} / \delta_{B L}\right)^{2}$ with boundary layer volume $\Omega_{B L} \sim L \delta_{B L}$. Hence, the boundary layer dissipation is expected to be dominant over the internal dissipation in the film as long as:

$$
\frac{\eta_{1} L}{\delta_{B L}}>\frac{\eta_{2} h_{f}}{L} \rightarrow L^{2}>\frac{\eta_{2}}{\eta_{1}} h_{f} \delta_{B L}
$$



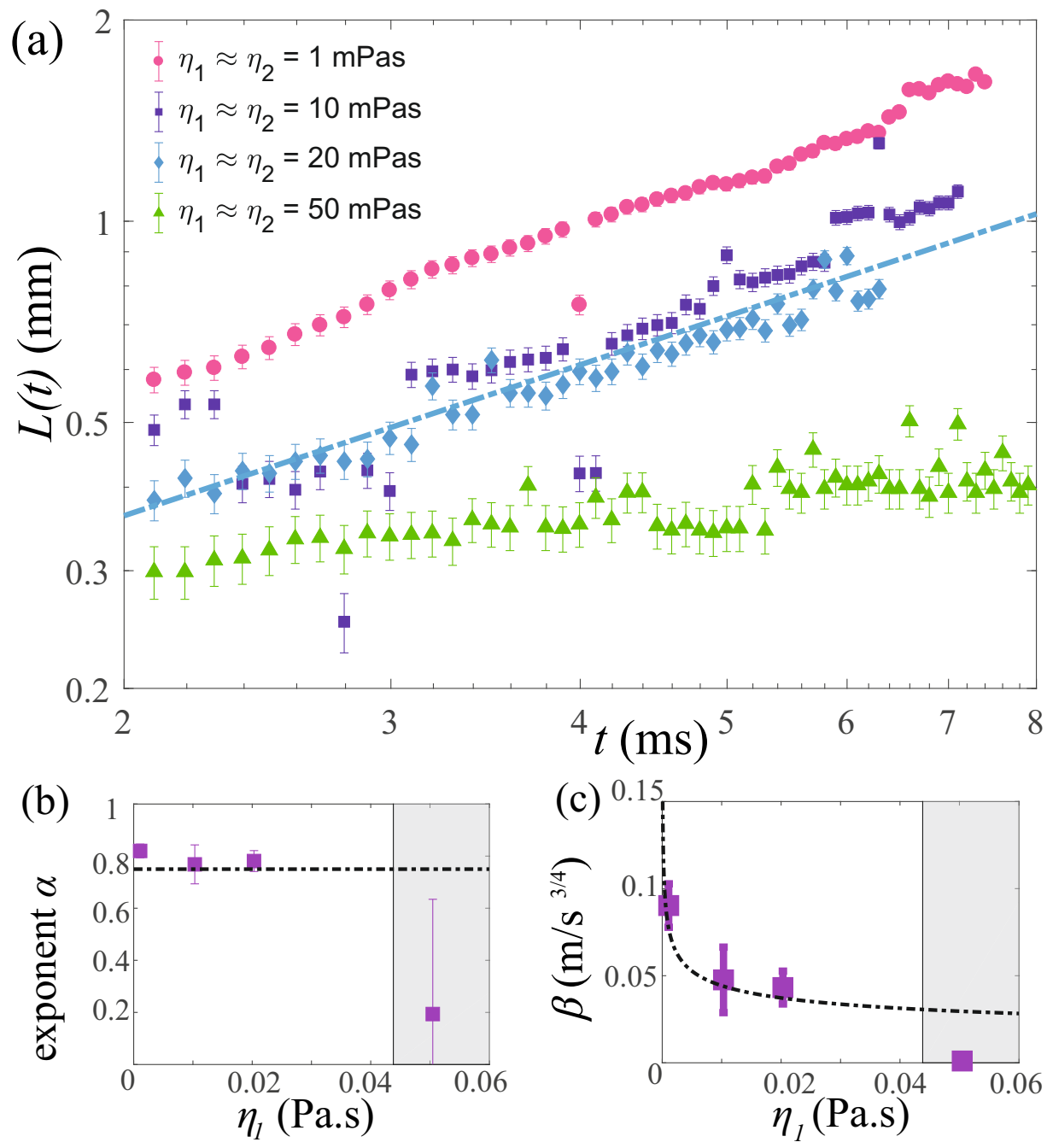

Figure 5.4: (a) Time evolution of the leading edge position $L(t)$ as a function of the viscosity, with $18.9 \leq \Delta \sigma \leq 23.2 \mathrm{mN} \mathrm{m}^{-1}$. The dash-dotted line indicates $L(t)=\beta t^{\alpha}$ with $\alpha=3 / 4$ and $\beta=0.6 \Delta \bar{\sigma}^{1 / 2}\left(\rho_{1} \eta_{1}\right)^{-1 / 4}$, as expected for spreading over a deep bath. (b) The spreading exponent $\alpha$ as a function of the viscosity; the dash-dotted line indicates $3 / 4$. (c) The prefactor $\beta$ as a function of the viscosity, with the dashed line indicating $0.6 \Delta \sigma^{1 / 2}\left(\rho_{1} \eta_{1}\right)^{-1 / 4}$. In (b,c) The shaded areas indicate $O h>0.2$ for our liquids. The error bars indicate a confidence interval of $95 \%$. 

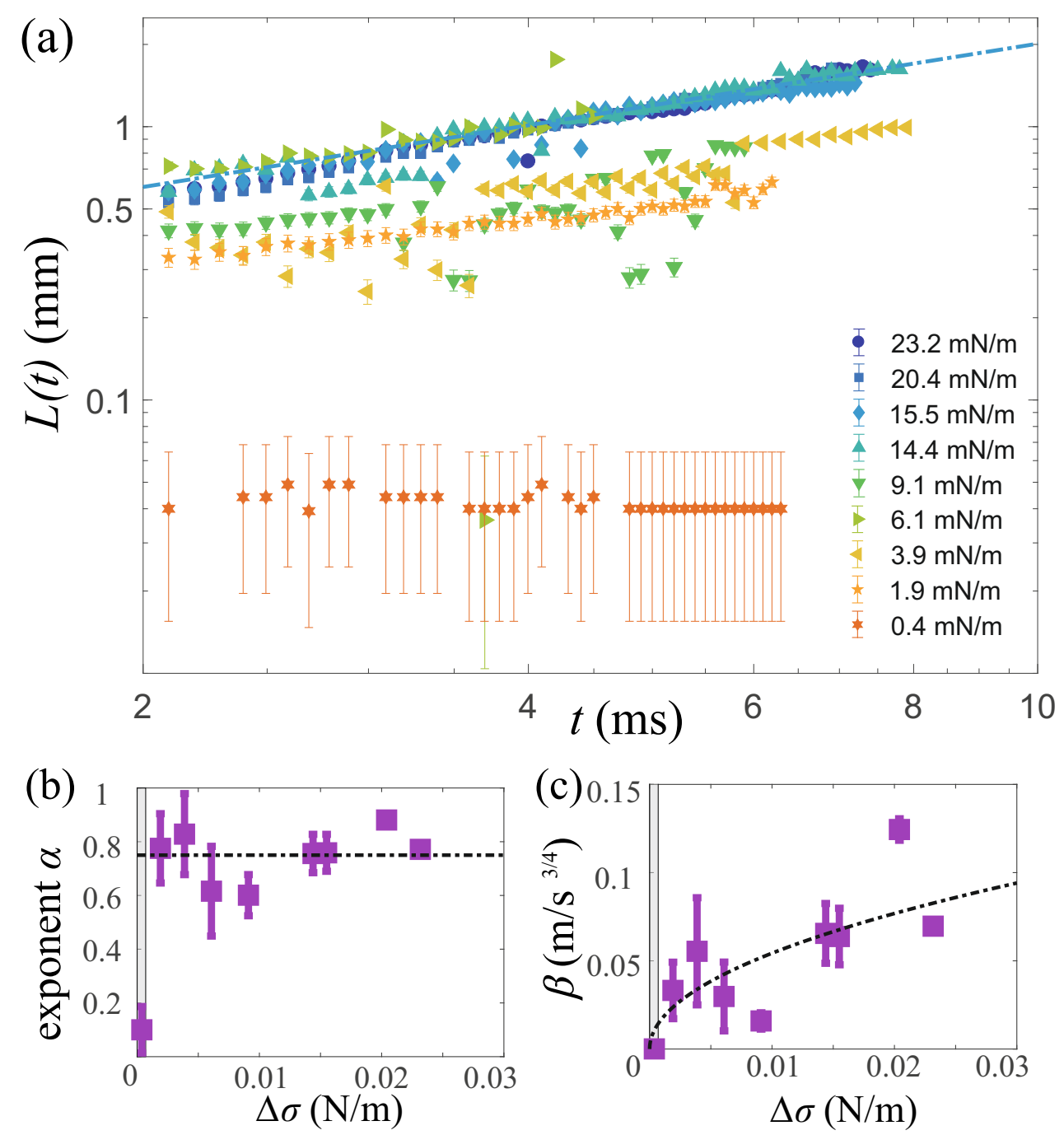

Figure 5.5: (a) Time evolution of $L(t)$ as a function of $\Delta \sigma$, with $\eta_{2}=1.5 \mathrm{mPa}$ and $1<\eta_{1} / \eta_{2}<1.9$. (b) The exponent $\alpha$ as a function of $\Delta \sigma$. (c) Prefactor $\beta$ as a function of $\Delta \sigma$. In (b,c), the shaded areas close to the origin indicate $O h>0.2$ and the dash-dotted lines correspond to those in figure 5.4. The error bars indicate a confidence interval of $95 \%$. 


\subsection{Results and discussion}

Assuming $L(t)=\beta t^{3 / 4}$ with $\beta \approx 0.05 \mathrm{~ms}^{-4 / 3}$ (as observed in figure 5.5), $\delta_{B L}=$ $\left(\eta_{1} t / \rho_{1}\right)^{1 / 2}$, a film thickness $h_{f}=100 \mu \mathrm{m}$ (comparable to the measurement threshold), and the most viscous film $\left(\eta_{1} / \eta_{2}=0.01\right)$, the transition to film-limited spreading would be expected after approximately $4 \mathrm{~ms}$. Indeed, a strong reduction of the spreading is observed for $t \approx 5 \mathrm{~ms}$. At earlier times, the measured spreading exponent $\alpha \approx 3 / 4$ suggests that spreading is limited by the boundary layer, but the strongly reduced prefactor shows that the film viscosity still has a strong influence. This behavior may reflect a transition between two different regimes, which deserves further attention as it has not yet been addressed.

As a final step, we rescale our results and compare these to Marangoni-driven spreading in other geometries, with different surfactants, and with immiscible liquids. The dimensionless spreading time and distance were formulated as proposed by [56]:

$$
\begin{aligned}
\tilde{t} & =\frac{t}{\eta_{1}^{3} / \Delta \sigma^{2} \rho_{1}}, \\
\tilde{L} & =\frac{L}{\eta_{1}^{2} / \Delta \sigma \rho_{1}} .
\end{aligned}
$$

Our measurements are generally described by

$$
\tilde{L}=0.6 \tilde{t}^{3 / 4},
$$

as plotted in figure 5.7. Surfactant-driven flows [40, 43] and immiscible drops with various viscosities $[42,56]$ are also well-captured by equation 5.5 . For unidirectional spreading, a higher constant in the range $0.66<k<2$, but typically $k \approx 1.39$, is expected $[6,41,56]$ and observed in figure $5.7[40,43,56]$ [42]. Note that this figure covers 10 orders of magnitude in dimensionless time $\tilde{t}$ and 7.5 orders of magnitude in dimensionless spreading distance $\tilde{L}$.

Our measurements with reduced exponents are still partly congruent to equation 5.5. Similarly, the measurements by Kim et al.[49] collapse onto this master curve for a bath with $\eta=5 \mathrm{mPa}$, whereas spreading over a bath with lower viscosity only initially follows equation 5.5 and then stops at later times. This transition to a spreading coefficient $\alpha=0$ was attributed to Marangoni-induced mixing [49], and may thus indicate a transition to miscibility-dominated effects. Our case may be essentially immiscible as (1) the diffusion length scale over the experimental duration of $10 \mathrm{~ms}\left(\delta_{D}=\left(D_{m} t\right)^{1 / 2} \approx 3 \mu \mathrm{m}\right.$ with $D_{m}$ the mass diffusivity) is much smaller than the thickness of the measured film and (2) a spreading exponent $\alpha \approx 3 / 4$ is typically observed. However, a prefactor $k \approx 0.88$ was reported [41] for immiscible liquids whereas we observe $k \approx 0.6$. This reduced value may indicate that miscibility has a minor but observable influence, as $k \approx 0.3$ was reported for miscible $[48,51]$ liquid pairs in which vortex formation slows down spreading. As these vortices were observed both in larger [48] and smaller [50] systems, vortices are likely to develop in 

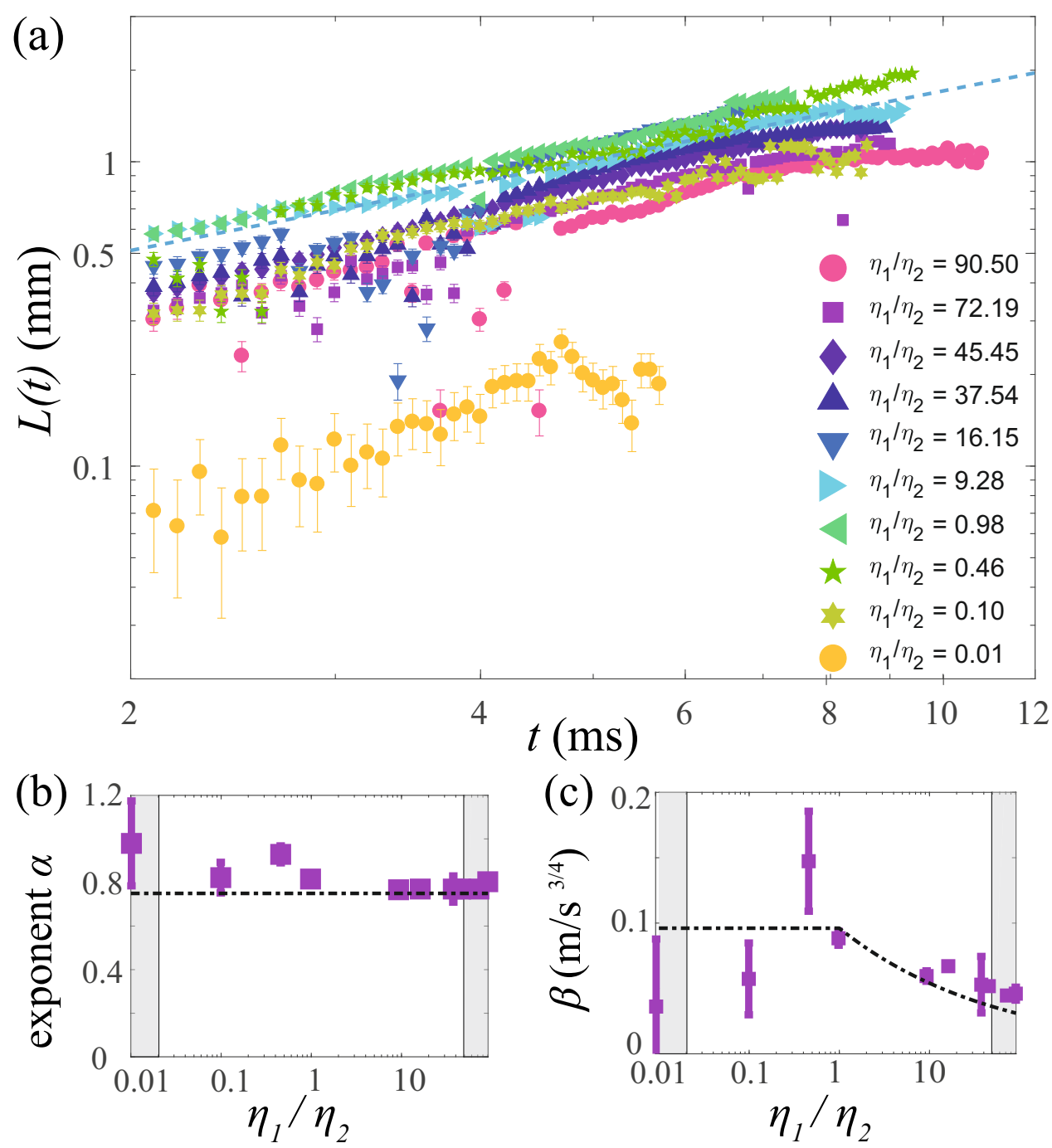

Figure 5.6: (a) Time evolution of the spreading edge $L(t)$ for various viscosity ratios $\eta_{1} / \eta_{2}$, with $19.8 \leq \Delta \sigma \leq 28.3 \mathrm{mNm}^{-1}$. For $\eta_{1} / \eta_{2}<1, \eta_{1}=1.5 \mathrm{mPa}$ and for $\eta_{1} / \eta_{2}>1$, $\eta_{2}=1.5 \mathrm{mPas}$ (b) Spreading exponent $\alpha$ and (c) prefactor $\beta$ as a function of $\eta_{1} / \eta_{2}$. In (b,c), the shaded areas indicate $O h>0.2$ (based on the highest viscosity) and the dash-dotted lines correspond to those in figure 5.4. The error bars indicate a confidence interval of $95 \%$. 


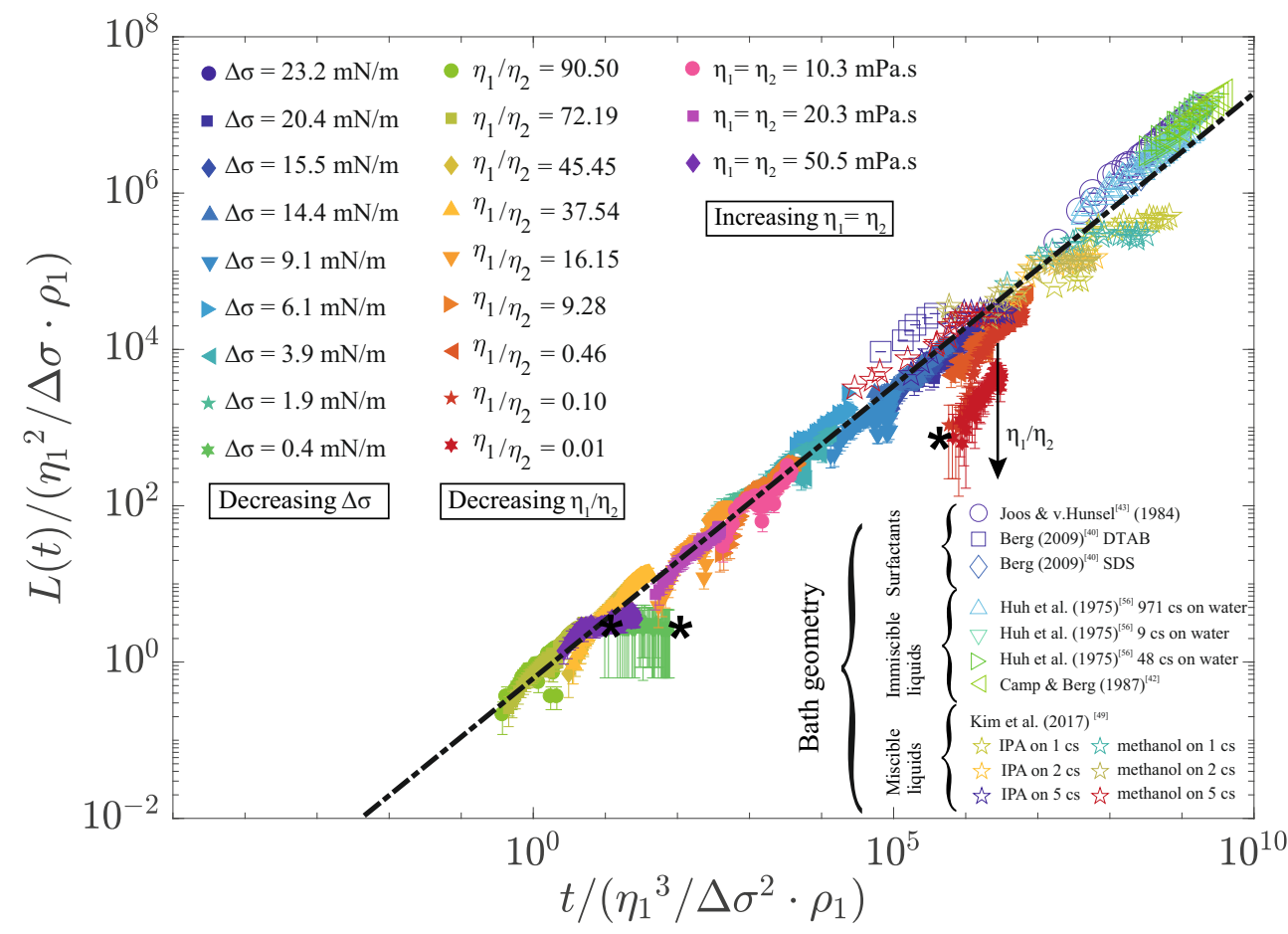

Figure 5.7: Rescaled spreading distance as a function of rescaled time, for our measurements and literature data for different geometries, miscibility, and surfactants [40, 42, 43, 49, 56]. The dash-dotted line indicates $\tilde{L}=0.6 \tilde{t}^{3 / 4}$, and the stars (*) indicate measurements for which $\mathrm{Oh}>0.2$.

our system as well. As the influence of miscibility cannot yet be determined from the literature, even for the flat geometry, future work will be required to investigate the details of this parameter.

Our measurements with $\eta_{1} / \eta_{2}<1$ do not collapse on equation 5.5, since the film viscosity was not included in the dimensionless variables. As discussed, the exact influence of the viscosity in the low- $\sigma$ film is still unclear. Measurements with a spreading exponent $\alpha=1 / 4$ were recently reported for larger ethanol-water systems, but could only be modeled by assuming a no-slip condition for the film [52]. This is a strong assumption, since the film was only in contact with a deep bath on the bottom and free-flowing air on the top. Therefore, the transition between film-limited and substrate-limited spreading as well as the spreading of ethanol over water also deserve future research.

We expected that the geometry would play an important role, as a toroidal convection pattern will develop within drop 1 [47] as shown in figure 8 of the supplementary materials. When this flow is fully developed, the shear profile may resemble spreading 
over a thin film on a solid substrate, for which $\alpha=1 / 2$ [57-59]. This transition to film-limited spreading may occur at the end of our temporal domain, as discussed in the supplementary section 0.5 . However, the observed temporally sustained spreading exponent $\alpha=3 / 4$ suggests that drop encapsulation can be modeled as a deep bath for most of our control parameters. Finally, our measurements confirm that the surrounding fluid plays an important role, as a constant spreading velocity $(\alpha=1)$ was reported for binary drops that were submerged in a liquid $[29,30]$.

\subsection{Conclusions}

The dynamics of Marangoni spreading were experimentally studied for miscible pendant drop pairs in the millimeter size range. Stroboscopic image sequences revealed the spreading distance as a function of time in a new temporal domain. The spreading distance is consistent with $L(t) \approx 0.6 \Delta \sigma^{1 / 2}(\rho \eta)^{-1 / 4} t^{3 / 4}$ for sufficiently low Ohnesorge numbers $(\mathrm{Oh} \lesssim 0.2)$, and moderate viscosity ratios $\left(0.1 \lesssim \eta_{1} / \eta_{2} \lesssim\right.$ 10). Marangoni-driven encapsulation is suppressed for $\mathrm{Oh} \gtrsim 0.2$, when viscous forces become comparable to capillary effects. Non-dimensionalizing our results and literature experiments for different surfactants, and miscibilities revealed that a universal power law $\tilde{L}=0.6 \tilde{t}^{3 / 4}$ reasonably captures all data over 10 orders of magnitude in dimensionless time, as shown in figure 5.7. This power law can therefore exploited to estimate the encapsulation time scale in a wide range of encapsulation applications.

Comparing our results to the literature revealed that Marangoni spreading of miscible liquids is not yet adequately understood, since the transition from essentially immiscible liquids (as observed here) to miscible liquids is unclear. Furthermore, the transition from boundary layer-dominated dissipation in the bath to film-dominated dissipation during elongation deserves attention. As the dynamics of the spreading film are challenging to visualize, especially at the early time scales, we expect that theory, numerics, and experiments will all be required to substantially advance the understanding of Marangoni spreading over the full temporal domain. 


\section{A Range of control parameters}

Table 5.1: Range of the material parameters. The properties of ink-containing liquids were measured as described below; literature values were taken for the other liquids [60-63].

\begin{tabular}{|c|c|c|c|c|c|}
\hline & \multicolumn{2}{|c|}{ drop 1} & \multicolumn{2}{|c|}{ drop 2} & \multirow{2}{*}{ 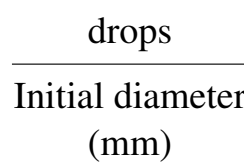 } \\
\hline & $\begin{array}{l}\text { Surface tension } \\
\quad\left(\mathrm{mN} \mathrm{m}^{-1}\right)\end{array}$ & $\begin{array}{c}\text { Viscosity } \\
\text { (mPas) }\end{array}$ & $\begin{array}{l}\text { Surface tension } \\
\quad\left(\mathrm{mNm}^{-1}\right)\end{array}$ & $\begin{array}{c}\text { Viscosity } \\
\text { (mPas) }\end{array}$ & \\
\hline & 46.2 to 72.8 & 1 to 100 & 22.9 to 46.2 & 1 to 100 & 2.00 to 2.92 \\
\hline Error & $5 \%$ & $2 \%$ & $5 \%$ & $2 \%$ & $0.1 \%$ \\
\hline
\end{tabular}

\section{B Surface tension measurements}

Surface tension was measured in triplo, using a pendant drop system (Data Physics OCA 15EC). The surface tension as a function of the ethanol percentage is shown in figure 5.8; the dependency of the surface tension on the glycerol percentage is shown in figure 5.9, and the time-insensitivity of the surface tension is shown in figure5.10.

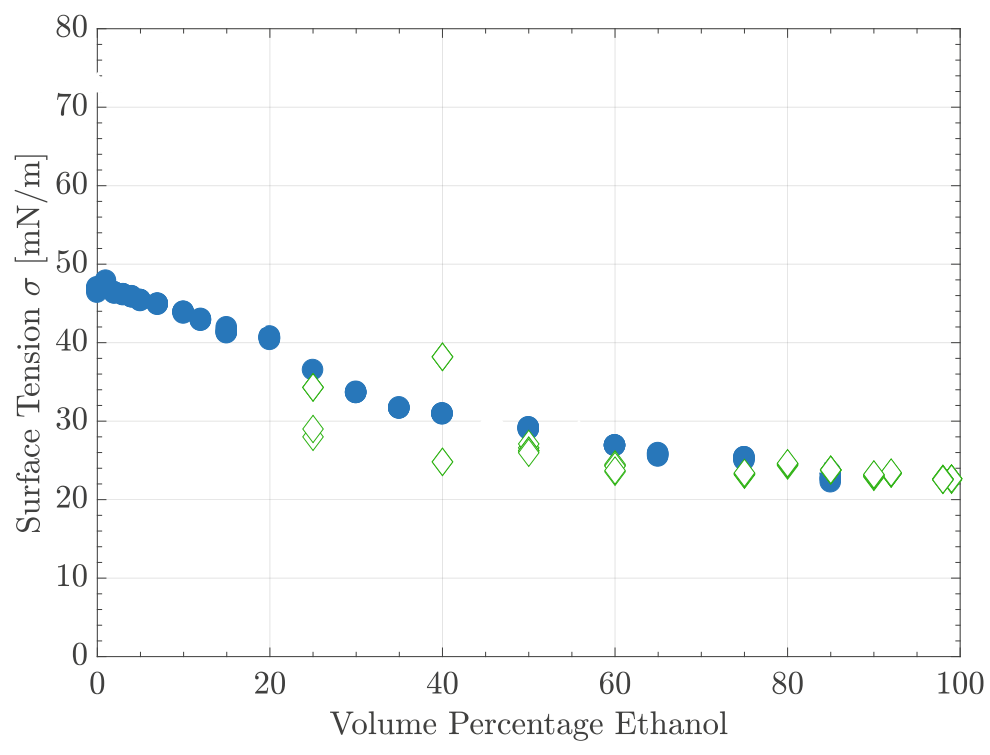

Figure 5.8: Surface tension of the water-ink-ethanol mixture as a function of the vol\% of ethanol (•). $15 \mathrm{vol} \%$ ink (Brother LC-800) was dissolved. Literature values of water-ethanol mixtures without ink are from [61] $(\diamond)$. 


\section{C Viscosity measurements}

Viscosity data was obtained using a rheometer (Anton Paar MCR 502), or from the literature, when indicated. The viscosity of the water-ethanol mixture is shown in figure 5.11. Figure 5.12 shows the viscosity of the water-glycerol mixture. To both compositions $15 \mathrm{vol} \%$ ink (Brother LC-800) is added as a colorant.

\section{D Optical penetration depth}

To determine the spreading rate, a threshold value for absorbed light has been chosen in the analysis. The transmission of light versus the optical penetration depth for the ink solutions are measured using a UV-VIS spectrometer (DR5000, Hach Lange), with pure air and a cuvette with water as references. The results are shown in figure 5.13. For 5 vol\% ink a range of transmission has been chosen between $0.025<$ $I / I_{0}<0.1$, corresponding to optical penetration depths of $51 \mu \mathrm{m}$ to $78 \mu \mathrm{m}$. The effect of the total transmittance threshold on the measurements of $L(t)$ is shown in figure 5.14. Increasing the transmittance leads to slightly lower penetration depth, therefore showing faster spreading. The effects in the ranges studied show the same exponents, $\alpha=0.77$, and prefactors, $0.45 \beta$, for penetration depths of $63 \mu \mathrm{m}$ and $78 \mu \mathrm{m}$. For the smaller penetration depth of $51 \mu \mathrm{m}$, the prefactor increases to 0.51 .

\section{E Boundary layer thickness}

The transition from the deep-bath regime to the thin-film regime can be expected as soon as a backflow will limit the development of the boundary layer in drop 1 . To first approximation, the viscous boundary layer cannot grow any further when $\delta_{B L}>D / 4$, as shown in Figure 5.15. The Blasius boundary layer thickness is described by $\delta_{B L}=(\eta t / \rho)^{1 / 2}$, as shown in figure 5.16b. Only for experiments with a highly viscous inner drop $\left(\eta_{1}>40 \mathrm{mPa}\right.$ s), the boundary layer reaches $D / 4$ as shown in Figure 5.16b. Indeed, in that case we observe a flattening of the spreading curve (Figure 5.16a) that is consistent with $\alpha=1 / 2$ as expected for thin-film spreading [40]. 
5.E. Boundary layer thickness

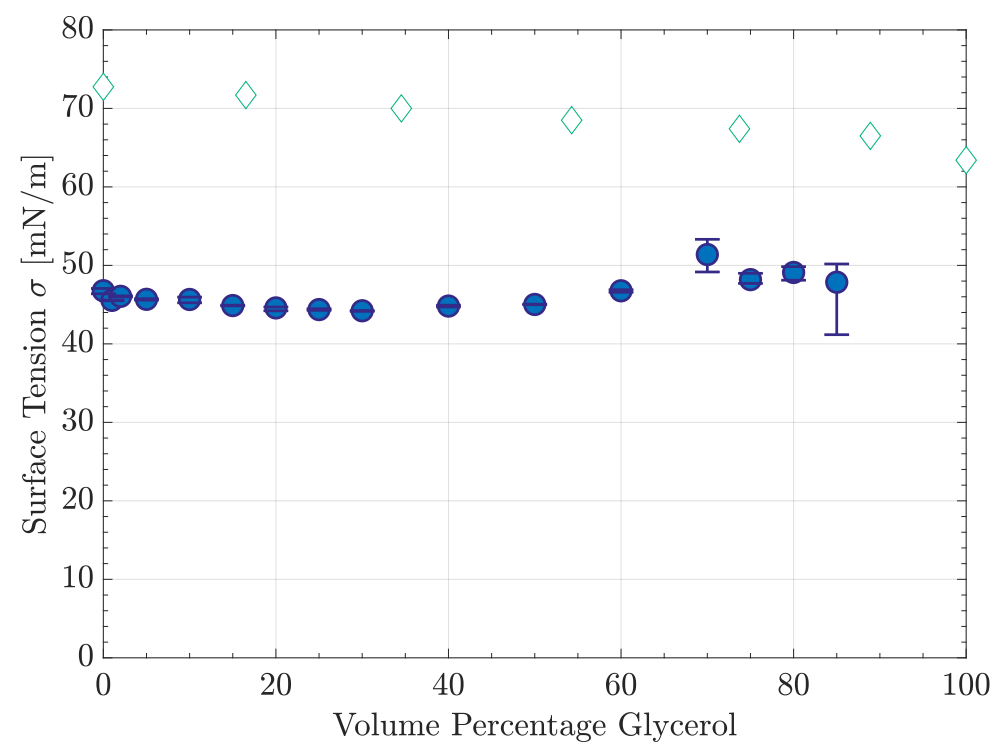

Figure 5.9: Surface tension of the water glycerol mixture, depending on the vol\% of glycerol. For the values used in the paper (•) 15 vol\% ink (Brother LC-800) was solved in the glycerol. Water-glycerol values are taken from (author?) [63] ( $\diamond)$.

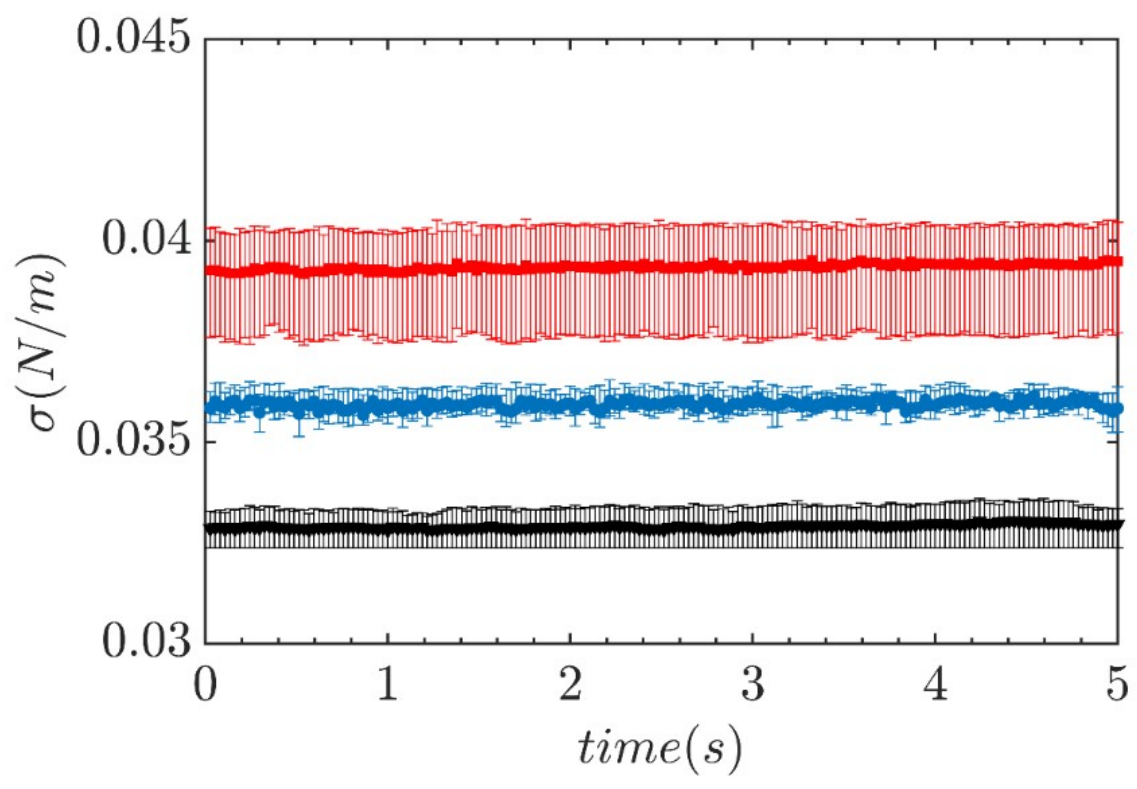

Figure 5.10: Surface tension of various mixtures as a function of time. $15 \%$ ink, $10 \%$ ethanol and $75 \%$ water (red), $100 \%$ ink (blue), $15 \%$ ink and $85 \%$ ethanol (black). 


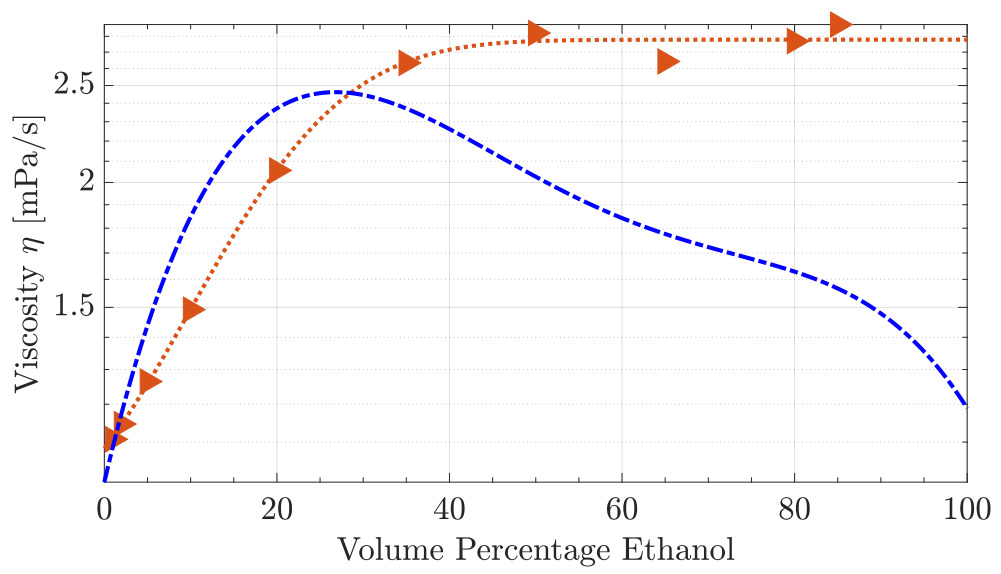

Ethanol with $15 \%$ vol Ink

$\eta=1.823+0.956 \operatorname{erf}(0.054 x-0.854)$

- - Khattab (2012): $\ln \eta=x_{W} \cdot \ln \eta_{w}+x_{E} \cdot \ln \eta_{e}+724.652 \frac{x_{W} \cdot x_{E}}{T}+729.357 \frac{x_{E} \cdot x_{W} \cdot\left(x_{W}-x_{E}\right)}{T}+976.05 \frac{x_{W} \cdot x_{E} \cdot\left(x_{W}-x_{E}\right)^{2}}{T}$

Figure 5.11: Viscosity of of the water ethanol mixture, depending on the vol\% of ethanol.

Measurements $(\bullet)$ and data fit (- - ). 15 vol\% ink (Brother LC-800) was solved in the ethanol. Water-ethanol mixtures from ref. [62] are shown by the blue line (-- - ).

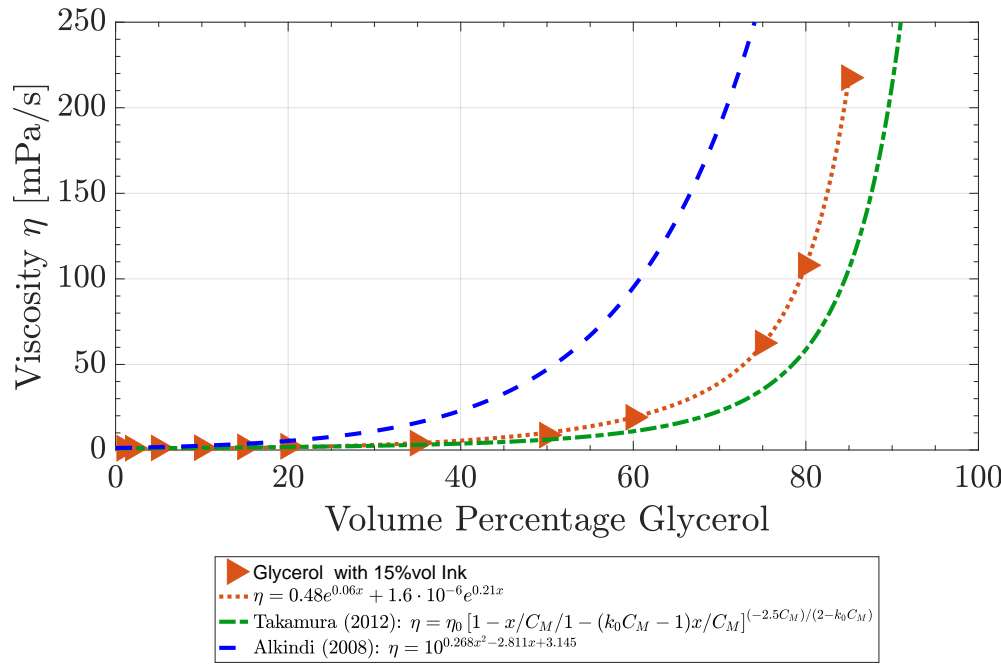

Figure 5.12: Viscosity of the water glycerol mixture as a function of the vol\% of glycerol. Measurements ( $\bullet$ ) and data fit (- - ). 15 vol\% ink (Brother LC-800) was solved in the glycerol mixture. Previously determined values for water-glycerol mixtures are from [63] (-.- ) and [61](-- -). 


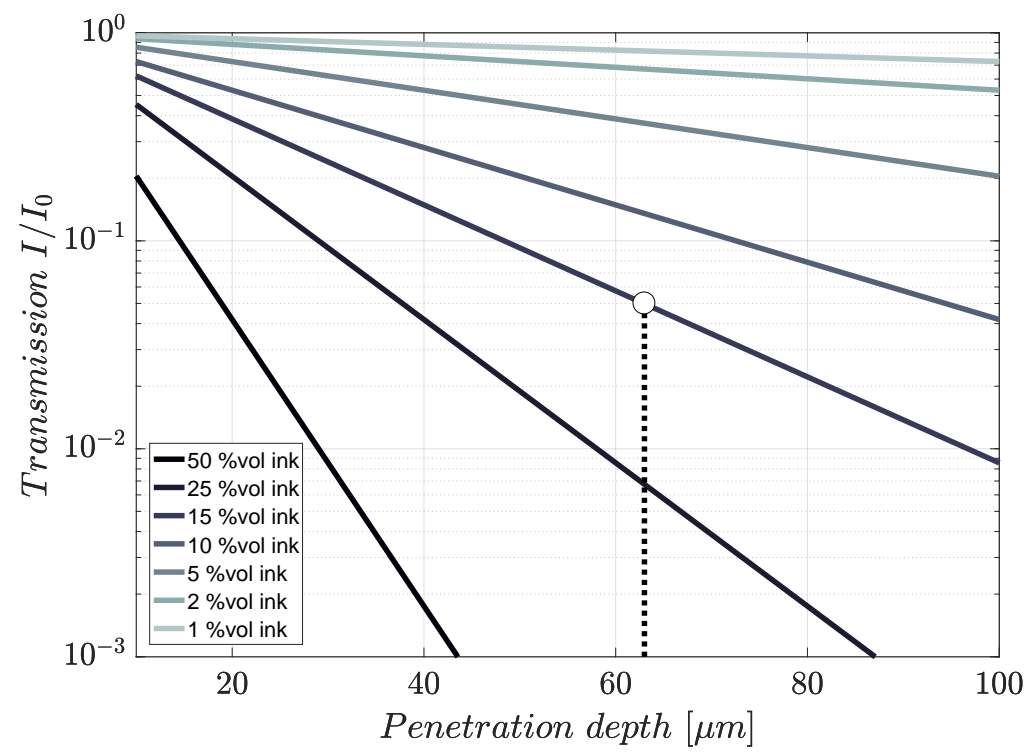

Figure 5.13: Optical transmittance of ink (Brother LC-800) in water solutions versus the penetration depth. $5 \%$ transmittance was chosen as the experimental threshold, corresponding to a penetration depth of $63 \mu \mathrm{m}$ shown by the symbol (o).

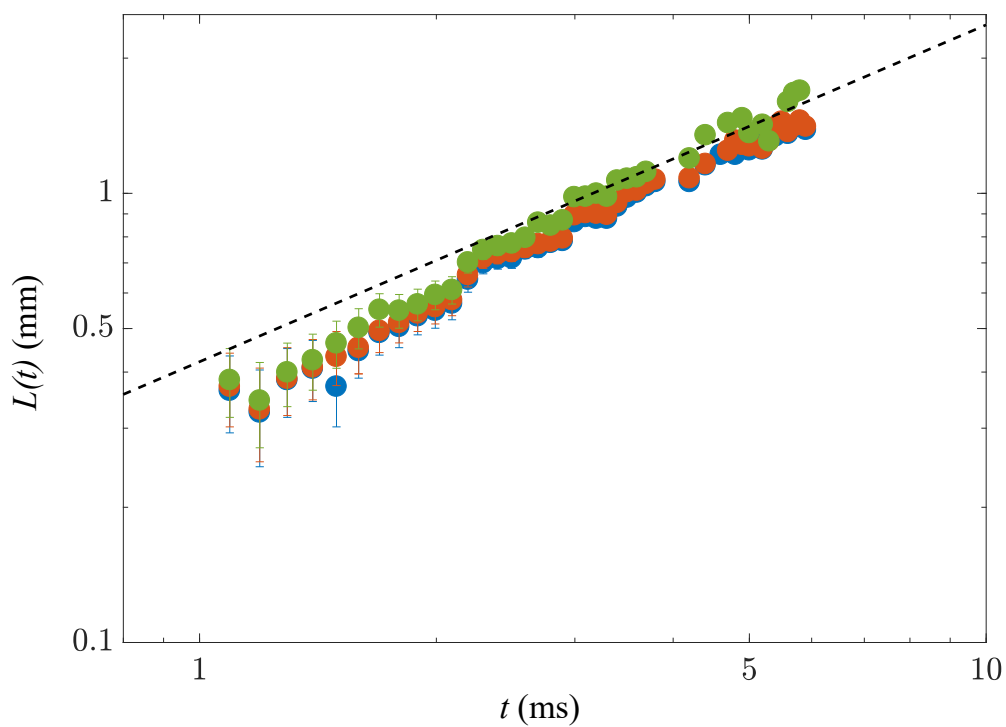

Figure 5.14: Effect of the threshold value on the measured spreading distance, $\eta_{1} \approx \eta_{2} \approx 1.5$ $\mathrm{mPas}, \Delta \sigma=32 \mathrm{mNm}^{-1}$. Optical penetration depths are $78 \mu \mathrm{m}(\mathrm{\circ}), 63 \mu \mathrm{m}(\mathrm{\circ})$, and $51 \mu \mathrm{m}(\bullet)$. The dashed line (- - ) indicates $L(t)=0.5 \beta t^{0.77}, \beta=\Delta \sigma^{1 / 2}\left(\rho_{1} \eta_{1}\right)^{-1 / 4}$. 


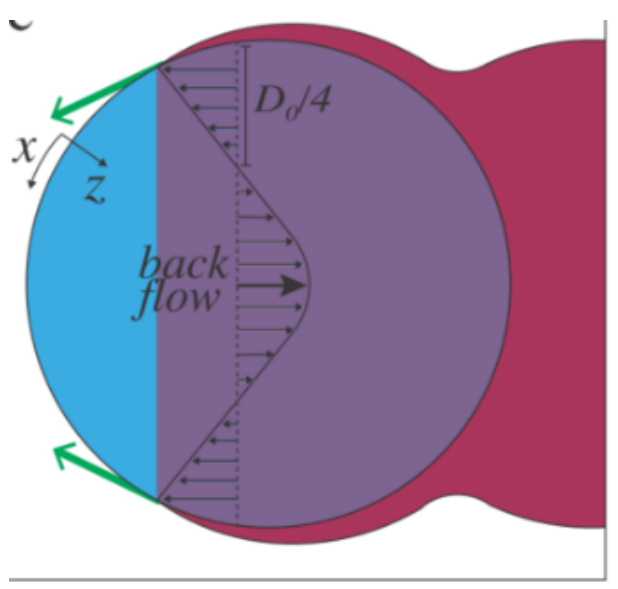

Figure 5.15: Indication of the flow profile in an encapsulatead droplet. The penetration depth of the boundary layer grow up to $\delta_{B L}>D / 4$, where it touches the backflow. (o).

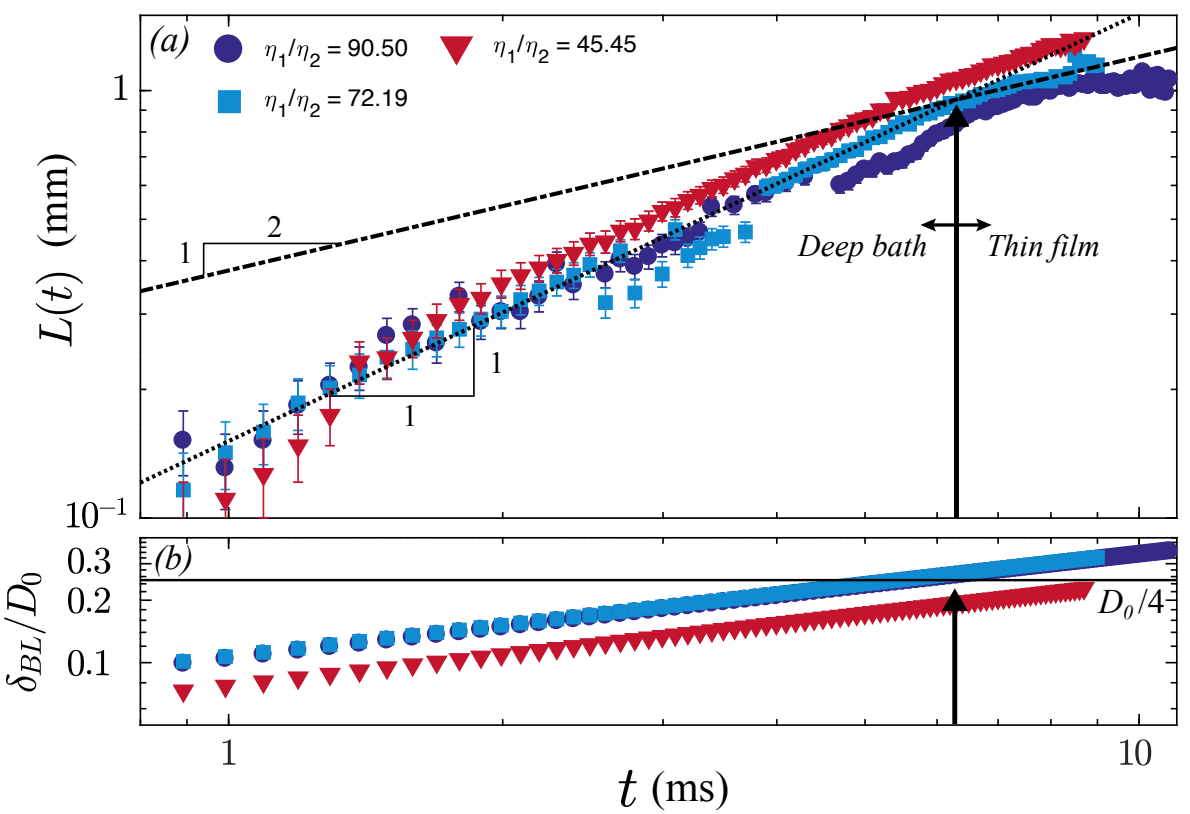

Figure 5.16: Temporal transition in spreading regimes; $(a)$ Spreading versus time for data with large $\eta_{1} / \eta_{2}$. Initial spreading scales as $L(t) \sim t^{\alpha}$ with $\alpha=1$. Later in time, a transition to $\alpha=0.5$ is visible. Both slopes are given by the dashed-dotted (1/2) and dotted line (1); $(b)$ Corresponding growth of relative boundary layer $\delta_{B L} / D_{0}$. Transition to $\alpha=0.5$ occurs around $\delta_{B L} / D_{0}=D_{0} / 4$, suggesting thin film-limited spreading when the boundary layer interacts with the toroidal vortices. 


\section{References}

[1] J. D. Paulsen, J. C. Burton, and S. R. Nagel, "Viscous to inertial crossover in liquid drop coalescence", Phys. Rev. Lett. 106, 114501 (2011).

[2] B. Franklin, W. Brownrigg, and M. Farish, "Of the Stilling of Waves by means of Oil. Extracted from Sundry Letters between Benjamin Franklin, LL. D. F. R. S. William Brownrigg, M. D. F. R. S. and the Reverend Mr. Farish", Philos. Trans. R. Soc. London 64, 445-460 (1774).

[3] L. Rayleigh, "Measurements of the Amount of Oil Necessary in Order to Check the Motions of Camphor upon Water", Proc. R. Soc. London 47, 364-367 (1889).

[4] J. A. Fay and D. P. Hoult, "Physical processes in the spread of oil on a water surface", (1971).

[5] D. P. Hoult, "Oil Spreading on the Sea", Annu. Rev. Fluid Mech. 4, 341-368 (1972).

[6] M. Foda and R. G. Cox, "The spreading of thin liquid films on a water-air interface", J. Fluid Mech. 101, 33-51 (1980).

[7] D. P. Gaver and J. B. Grotberg, "The dynamics of a localized surfactant on a thin film”, J. Fluid Mech. 213, 127-148 (1990).

[8] R. V. Craster and O. K. Matar, "Dynamics and stability of thin liquid films", Rev. Mod. Phys. 81, 1131-1198 (2009).

[9] N. D. Denkov, "Mechanisms of foam destruction by oil-based antifoams", Langmuir 20, 9463-9505 (2004).

[10] Y. Tian, X. Wei, Z. J. Wang, P. Pan, F. Li, D. Ling, Z. L. Wu, and Q. Zheng, “A facile approach to prepare tough and responsive ultrathin physical hydrogel films as artificial muscles", ACS Appl. Mater. Interface 9, 34349-34355 (2017).

[11] Y. Yeo, O. A. Basaran, and K. Park, "A new process for making reservoir-type microcapsules using ink-jet technology and interfacial phase separation", J. Control. Release 93, 161-173 (2003).

[12] Y. Yeo, A. U. Chen, O. A. Basaran, and K. Park, "Solvent exchange method: A novel microencapsulation technique using dual microdispensers", Pharm. Res. 21, 1419-1427 (2004).

[13] B. Duan, L. A. Hockaday, K. H. Kang, and J. T. Butcher, "3D Bioprinting of heterogeneous aortic valve conduits with alginate/gelatin hydrogels", J. Biomed. Mater. Res. - Part A 101 A, 1255-1264 (2013). 
[14] A. T. Ten Cate, C. H. Gaspar, H. L. K. Virtanen, R. S. A. Stevens, R. B. J. Koldeweij, J. T. Olkkonen, C. H. A. Rentrop, and M. H. Smolander, "Printed electronic switch on flexible substrates using printed microcapsules", J. Mater. Sci. 49, 5831-5837 (2014).

[15] N. Blanco-Pascual, R. B. J. Koldeweij, R. S. A. Stevens, M. P. Montero, M. C. Gómez-Guillén, and A. T. Cate, "Peptide Microencapsulation by Core-Shell Printing Technology for Edible Film Application", Food Bioprocess Technol. 7, 2472-2483 (2014).

[16] M. Hayakawa, H. Onoe, K. H. Nagai, and M. Takinoue, "Complex-shaped threedimensional multi-compartmental microparticles generated by diffusional and Marangoni microflows in centrifugally discharged droplets", Sci. Rep. 6, 20793 (2016).

[17] T. Kamperman, V. D. Trikalitis, M. Karperien, C. W. Visser, and J. Leijten, "Ultrahigh-Throughput Production of Monodisperse and Multifunctional Janus Microparticles Using in-Air Microfluidics", ACS Appl. Mater. Interfaces 10, 23433-23438 (2018).

[18] C. W. Visser, T. Kamperman, L. P. Karbaat, D. Lohse, and M. Karperien, "Inair microfluidics enables rapid fabrication of emulsions, suspensions, and 3D modular (bio)materials", Sci. Adv. 4, eaao1175 (2018).

[19] R. H. Chen and C. T. Chen, "Collision between immiscible drops with large surface tension difference: Diesel oil and water", Exp. Fluids 41, 453-461 (2006).

[20] R. H. Chen, "Diesel-diesel and diesel-ethanol drop collisions", Appl. Therm. Eng. 27, 604-610 (2007).

[21] C. Planchette, E. Lorenceau, and G. Brenn, "Binary collisions of immiscible liquid drops for liquid encapsulation”, Fluid Dyn. Mater. Process. 7, 279-302 (2011).

[22] C. Focke and D. Bothe, "Direct numerical simulation of binary off-center collisions of shear thinning droplets at high Weber numbers", Phys. Fluids 24, 73105 (2012).

[23] C. Planchette, S. Petit, H. Hinterbichler, and G. Brenn, "Collisions of drops with an immiscible liquid jet”, Phys. Rev. Fluids 3, 93603 (2018).

[24] D. Liu, P. Zhang, C. K. Law, and Y. Guo, "Collision dynamics and mixing of unequal-size droplets”, Int. J. Heat Mass Transf. 57, 421-428 (2013). 
[25] C. Focke, M. Kuschel, M. Sommerfeld, and D. Bothe, "Collision between high and low viscosity droplets: Direct Numerical Simulations and experiments", Int. J. Multiph. Flow 56, 81-92 (2013).

[26] S. T. Thoroddsen, B. Qian, T. G. Etoh, and K. Takehara, "The initial coalescence of miscible drops", Phys. Fluids 19, 72110 (2007).

[27] F. Blanchette, L. Messio, and J. W. M. Bush, "The influence of surface tension gradients on drop coalescence", Phys. Fluids 21, 72107 (2009).

[28] K. Sun, P. Zhang, Z. Che, and T. Wang, "Marangoni-flow-induced partial coalescence of a droplet on a liquid/air interface", Phys. Rev. Fluids 3, 23602 (2018).

[29] E. Nowak, N. M. Kovalchuk, Z. Che, and M. J. H. Simmons, "Effect of surfactant concentration and viscosity of outer phase during the coalescence of a surfactantladen drop with a surfactant-free drop", Colloids Surfaces A Physicochem. Eng. Asp. 505, 124-131 (2016).

[30] E. Nowak, Z. Xie, N. M. Kovalchuk, O. K. Matar, and M. J. H. Simmons, "Bulk advection and interfacial flows in the binary coalescence of surfactant-laden and surfactant-free drops", Soft Matter 13, 4616-4628 (2017).

[31] F. Blanchette, "Simulation of mixing within drops due to surface tension variations", Phys. Rev. Lett. 105, 13-16 (2010).

[32] C. Vernay, L. Ramos, and C. Ligoure, "Bursting of Dilute Emulsion-Based Liquid Sheets Driven by a Marangoni Effect", Phys. Rev. Lett. 115, 198302 (2015).

[33] D. G. Suciu, O. Smigelschi, and E. Ruckenstein, "Some experiments on the Marangoni effect”, AIChE J. 13, 1120-1124 (1967).

[34] D. G. Suciu, O. Smigelschi, and E. Ruckenstein, "On the structure of dissolving thin liquid films", AIChE J. 15, 686-689 (1969).

[35] D. G. Suciu, O. Smigelschi, and E. Ruckenstein, "The spreading of liquids on liquids”, J. Colloid Interface Sci. 33, 520-528 (1970).

[36] E. Ruckenstein, O. Smigelschi, and D. G. Suciu, "A steady dissolving drop method for studying the pure Marangoni effect", Chem. Eng. Sci. 25, 1249-1254 (1970).

[37] O. E. Jensen, "The Spreading of Insoluble Surfactant at the Free Surface of a Deep Fluid Layer", J. Fluid Mech. 293, 349-378 (1995). 
[38] J. A. Fay, "The Spread of Oil Slicks on a Calm Sea", in Oil Sea, 53-63 (Springer US, Boston, MA) (1969).

[39] P. Joos and J. Pintens, "Spreading kinetics of liquids on liquids", J. Colloid Interface Sci. 60, 507-513 (1977).

[40] S. Berg, "Marangoni-driven spreading along liquid-liquid interfaces", Phys. Fluids 21, 32105 (2009).

[41] A. D. Dussaud and S. M. Troian, "Dynamics of spontaneous spreading with evaporation on a deep fluid layer", Phys. Fluids 10, 23-38 (1998).

[42] D. W. Camp and J. C. Berg, "Spreading of oil on water in the surface-tension regime", J. Fluid Mech. 184, 445-462 (1987).

[43] P. Joos and J. Van Hunsel, "Spreading of aqueous surfactant solutions on organic liquids", J. Colloid Interface Sci. 106, 161-167 (1985).

[44] V. Bergeron and D. Langevin, "Monolayer spreading of polydimethylsiloxane oil on surfactant solutions", Phys. Rev. Lett. 76, 3152-3155 (1996).

[45] M. Roché, Z. Li, I. M. Griffiths, S. Le Roux, I. Cantat, A. Saint-Jalmes, and H. A. Stone, "Marangoni flow of soluble amphiphiles", Phys. Rev. Lett. 112, 208302 (2014).

[46] B. Wang and S. A. Socolofsky, "On the bubble rise velocity of a continually released bubble chain in still water and with crossflow", Phys. Fluids 27, 103301 (2015).

[47] V. V. Tarasov, N. F. Kovalenko, G. S. Shcherbakova, and D. Zhang, "Linear and radial marangoni flows of surfactants", Theor. Found. Chem. Eng. 40, 111-115 (2006).

[48] M. Santiago-Rosanne, M. Vignes-Adler, and M. G. Velarde, "On the spreading of partially miscible liquids", J. Colloid Interface Sci. 234, 375-383 (2001).

[49] H. Kim, K. Muller, O. Shardt, S. Afkhami, and H. A. Stone, "Solutal Marangoni flows of miscible liquids drive transport without surface contamination", Nat. Phys. 13, 1105-1110 (2017).

[50] F. Taherian, V. Marcon, E. Bonaccurso, and N. F. A. van der Vegt, "Vortex formation in coalescence of droplets with a reservoir using molecular dynamics simulations", J. Colloid Interface Sci. 479, 189-198 (2016). 
References

[51] H. Kim, J. Lee, T. H. Kim, and H. Y. Kim, "Spontaneous Marangoni Mixing of Miscible Liquids at a Liquid-Liquid-Air Contact Line", Langmuir 31, 8726-8731 (2015).

[52] R. Dandekar, A. Pant, and B. A. Puthenveettil, "Film spreading from a miscible drop on a deep liquid layer", J. Fluid Mech. 829, 304-327 (2017).

[53] L. Bacri and G. Debrégeas, "Experimental Study of the Spreading of a Viscous Droplet on a Nonviscous Liquid", Langmuir 12, 6708-6711 (1996).

[54] G. M. Schmid, R. M. Hurd, and E. S. Snavely, "Effects of Electrostatic Fields on the Surface Tension of Salt Solutions", J. Electrochem. Soc. 109, 852 (1962).

[55] E. K. Mann and D. Langevin, "Poly (dimethylsiloxane) molecular layers at the surface of water and of aqueous surfactant solutions", Langmuir 7, 1112-1117 (1991).

[56] C. Huh, M. Inoue, and S. G. Mason, "Uni-directional spreading of one liquid on the surface of another", Can. J. Chem. Eng. 53, 367-371 (1975).

[57] J. Ahmad and R. S. Hansen, "A simple quantitative treatment of the spreading of monolayers on thin liquid films", J. Colloid Interface Sci. 38, 601-604 (1972).

[58] J. F. Hernández-Sánchez, A. Eddi, and J. H. Snoeijer, "Marangoni spreading due to a localized alcohol supply on a thin water film", Phys. Fluids 27, 32003 (2015).

[59] M. Jehannin, S. Charton, S. Karpitschka, T. Zemb, H. Möhwald, and H. Riegler, "Periodic Precipitation Patterns during Coalescence of Reacting Sessile Droplets", Langmuir 31, 11484-11490 (2015).

[60] G. Vazquez, E. Alvarez, and J. M. Navaza, "Surface tension of alcohol + water from 20 to 50 C", J. Chem. Eng. Data 40, 611-614 (1995).

[61] A. S. Alkindi, Y. M. Al-Wahaibi, and A. H. Muggeridge, "Physical properties (density, excess molar volume, viscosity, surface tension, and refractive index) of ethanol + glycerol", J. Chem. Eng. Data 53, 2793-2796 (2008).

[62] I. S. Khattab, F. Bandarkar, M. A. A. Fakhree, and A. Jouyban, "Density, viscosity, and surface tension of water+ethanol mixtures from 293 to 323K", Korean J. Chem. Eng. 29, 812-817 (2012).

[63] K. Takamura, H. Fischer, and N. R. Morrow, "Physical properties of aqueous glycerol solutions", J. Pet. Sci. Eng. 98-99, 50-60 (2012). 



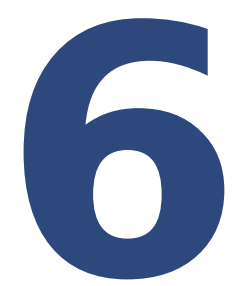

\section{A local curve fitting method to model variable surface tension *}

A novel methodology for the inclusion of variable surface tension acceleration in a geometrical Volume-of-Fluid method is developed. This numerical method provides an accurate method for computing surface tension gradients based on the local reconstruction of the interface. As such, it is applicable to both temperature and concentration gradients. The method is implemented in the open source solver of Basilisk. Two cases for validation are provided, the spreading of two liquids on top of each other and the migration of a droplet in a surface tension gradient field. Comparison of the numerical method to analytical results for these situations shows that the method accurately calculates liquid flow driven by variations in surface tension.

*This chapter will be written into an article that is suitable for publication by R.B.J. Koldeweij, J.J. Verschuur, R.M. van der Meer and D. Lohse 


\subsection{Introduction}

Flows induced by a spatial variation of surface tension, the Marangoni effect, can be caused by surfactants or gradients in temperature or concentration. These types of flow are of importance in various contexts, such as oil spills on the sea [1-5], pulmonary surfactant replacement therapy $[6,7]$, or encapsulation of droplets [814]. Several numerical methods for studying variable surface tension have been developed, including level-set [15], marker particles [16, 17], diffuse interface [18$20]$ and Volume-of-Fluid ( $\mathrm{VoF}$ ) methods [21-25]. Recent improvements on the VoF method, with the addition of height functions [26, 27], have made it a robust method for modelling interfacial flows with evolving interfaces [28-30]. A challenge on including variable surface tension effects into the VoF method is that the value for surface tension needs to be approximated at the interface. The approximation methods have limited the application variable surface tension methods to temperature gradients $[23,24]$ or concentration gradients due to surfactants [21, 25]. Recently, a method was developed that can be applied to both temperature and concentration gradients [31]. This method makes use of cell-centered values of the surface tension in the cells that contains the interface. Furthermore, it averages the surface tension value between multiple cells in a single column that contain the interface.

Here, we present a method that locally reconstructs the interface. We use this to interpolate the surface tension values of the neighbouring cells onto the positions where the interface crosses the cell faces. The variation of surface tension between the cell faces give a natural choice for the surface tension gradient. This method is usable interchangeably for both concentration and temperature gradients. We extend the open source solver BASILISK, which includes the Continuum Surface Force (CSF) [32] implementation of the surface tension force based on the height function algorithm.

This chapter is organized as follows: In section 6.2, the numerical implementation is described. Then this implementation is tested in section 6.3 , followed by the conclusions in section 6.4.

\subsection{Numerical method}

Describing Marangoni effects on liquid-liquid interfaces requires detailed calculation of the flow field near this interface. Therefore we use the open source solver BASILISK to simulate the general flow behavior. BASILISK solves the Navier-Stokes equations with the Volume of Fluid (VoF) method using adaptive grid refinement and is the successor of GERRIS [26, 33].

The governing equations to calculate the liquid-liquid flow behaviour are the mass conservation and incompressible Navier-Stokes equations, 


$$
\begin{aligned}
\nabla \cdot \mathbf{u} & =0 \\
\partial_{t} \mathbf{u}+(\vec{u} \cdot \nabla) \vec{u} & =\frac{1}{\rho}[-\nabla p+\nabla \cdot(2 \mu \overline{\mathbf{D}})]+\mathbf{a} .
\end{aligned}
$$

In these equations, $\mathbf{u}$ and $p$ represents the velocity and the pressure respectively. In equation $6.2, D$ represents the deformation tensor given by $\overline{\mathbf{D}}=\left[\nabla \mathbf{u}+\nabla \mathbf{u}^{T}\right] / 2$. The acceleration a contains the accelerations coming from the external forces, such as surface tension. The density $\rho$ and viscosity $\mu$ are calculated for two fluids by a colour function, in the form:

$$
\rho(c)=c \rho_{1}+(1-c) \rho_{2},
$$

where $c$ is 1 for cells containing the first fluid and 0 for cells containing the second fluid. The interface between the fluids is given by values of $f$ in between 0 and 1 $[26,34]$.

The acceleration due to surface tension $\sigma$ is split into a tangential and normal direction to the interface, as:

$$
\mathbf{a}_{s}=\sigma \kappa \delta_{s} \mathbf{n}+\delta_{s} \nabla_{s} \sigma
$$

where $\nabla_{s} u=\nabla u-\mathbf{n}(\mathbf{n} \cdot \nabla u)$ is the surface gradient, $\kappa$ is the curvature of the surface and $n$ is the normal component to the surface. The Dirac distribution function $\delta_{s}$ assures that the surface tension gradient is only located on the interface.

The normal component of surface tension acceleration is implemented in Basilisk using the Continuum-Surface-Force (CSF) method of Ref. [32]:

$$
\mathbf{a}_{s n}=\sigma \kappa \delta_{s} \mathbf{n} \approx \sigma \kappa \nabla c
$$

We write the tangential surface tension acceleration in a single cell, $\mathscr{C}$ as:

$$
\mathbf{a}_{s t}=\frac{1}{\Delta^{2}} \iint_{\mathscr{C}} \delta_{S} \nabla_{s} \sigma \mathrm{d} x \mathrm{~d} y=\frac{1}{\Delta^{2}} \int_{S} \frac{\mathrm{d} \sigma}{\mathrm{d} s} \mathbf{t} \mathrm{d} s,
$$

where $S$ is the part of the interface that is inside the cell. $\mathbf{t}$ lies along the curve of the interface. Assuming that the surface tension gradient $\frac{\mathrm{d} \sigma}{\mathrm{d} s} \approx\left(\sigma_{\text {right }}-\sigma_{\text {left }}\right) / \ell$ is constant in each finite volume cell, with $\ell$ the length of the interface in the cell and $\sigma_{\text {left,right }}$ the surface tension at the outermost points of the interface in a numerical cell, equation 6.6 can be written as:

$$
\mathbf{a}_{s t}=\frac{1}{\Delta^{2}}\left[\sigma_{\text {right }}-\sigma_{\text {left }}\right] \mathbf{t}
$$




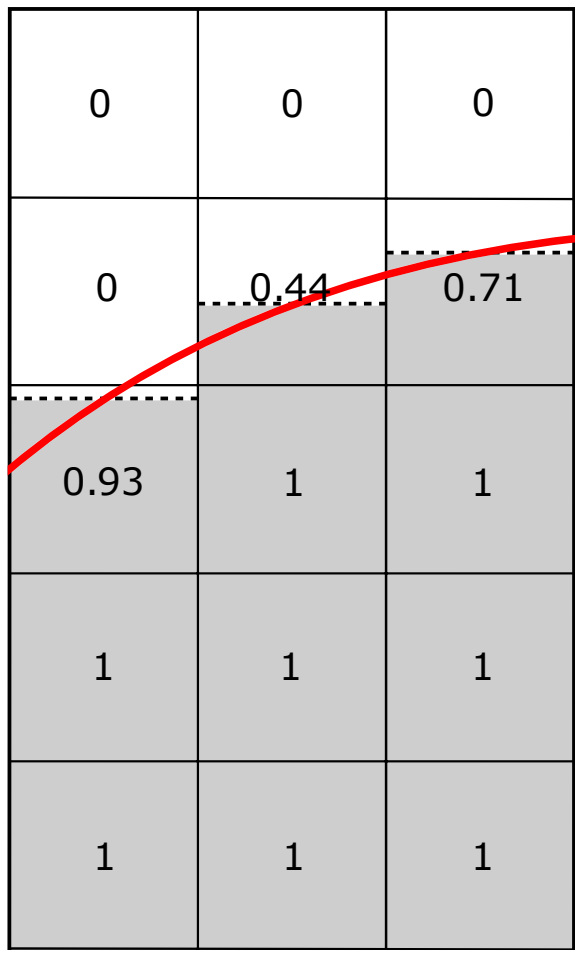

(a)

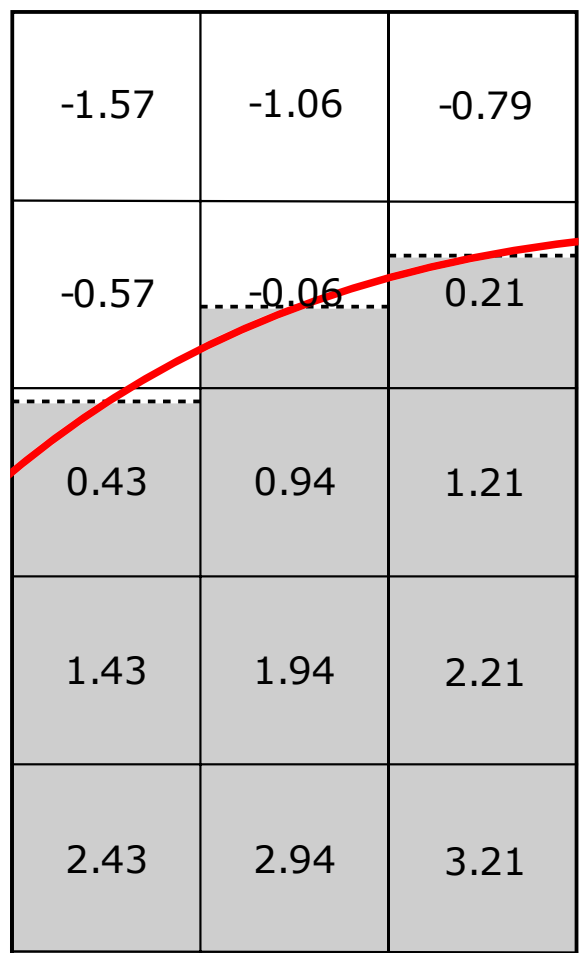

(b)

Figure 6.1: Stencil of the cells close to the interface of a tracer fluid. The numbers in the left image contain the $\mathrm{VoF}$ fraction $c$. The right image contains the corresponding height function values in vertical direction.

\subsubsection{Interface reconstruction}

The accurate calculation of the tangential component to the interface is critical to obtain the correct Marangoni stress. Therefore, an accurate, local description of the interface is required. We make use of a height function calculation to locally reconstruct the interface. Height functions are a discrete approximation of the interface height by locally summing the values of the VoF fractions [35], see figure 6.1. The method to find the height functions is given in Ref. [26]. To locally reconstruct the interface, we first determine the slope in both the $x$-direction and $y$-direction for each cell that contains the interface. Then we construct a second order polynomial fit in the direction of the smallest slope. However, the height function might not always exist in both directions for each cell, see figure 6.2a, in which case only the direction where the height function values do exist is used. Here we show an explanation of the fitting procedure based on the polynomial for the $y$-direction. 


\begin{tabular}{|c|c|c|c|c|}
\hline$?$ & $\begin{array}{c}-1.58 \\
?\end{array}$ & $\begin{array}{c}-0.87 \\
?\end{array}$ & $\begin{array}{c}-0.53 \\
?\end{array}$ & $\begin{array}{c}-0.38 \\
?\end{array}$ \\
\hline$\stackrel{?}{1.90}$ & -0.58 & & $\begin{array}{r}0.47 \\
-1.10\end{array}$ & $\begin{array}{r}0.62 \\
-2.10\end{array}$ \\
\hline $0 . \stackrel{?}{65}$ & $\begin{array}{l}0.42 \\
-0.35\end{array}$ & $\begin{array}{r}1.13 \\
-1.35\end{array}$ & $\begin{array}{r}1.47 \\
-2.35\end{array}$ & $\begin{array}{r}0.62 \\
-3.35\end{array}$ \\
\hline $0.1 \mathrm{f}$ & $\begin{array}{r}1.42 \\
-0.38\end{array}$ & $\begin{array}{r}2.13 \\
-1.38\end{array}$ & $\begin{array}{r}2.47 \\
-2.38\end{array}$ & $\begin{array}{r}0.62 \\
-3.38\end{array}$ \\
\hline$-d ? \cdot 17$ & $\begin{array}{r}2.42 \\
-1.17\end{array}$ & $\begin{array}{r}3.13 \\
-2.17\end{array}$ & $\begin{array}{r}3.47 \\
-3.17\end{array}$ & $\begin{array}{r}0.62 \\
-4.17\end{array}$ \\
\hline
\end{tabular}

(a)

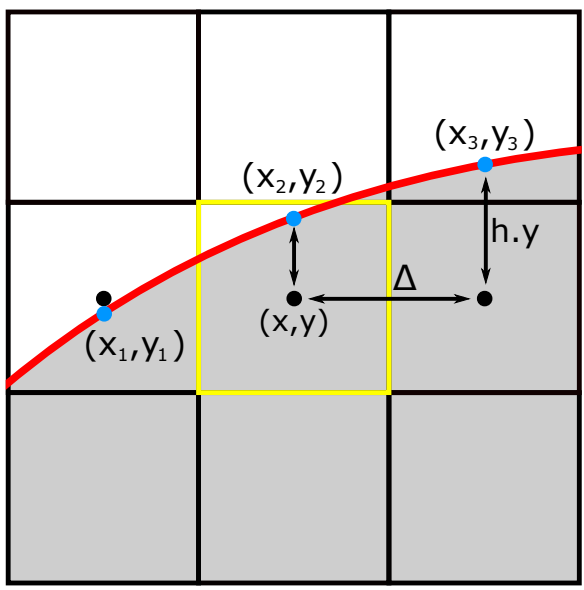

(b)

Figure 6.2: Method for the interface reconstruction (a) The height value data, with in green (top) the data in the $\mathrm{y}$ direction and in red (bottom) the data in the $\mathrm{x}$ direction for each cell. Question marks indicate that there is no height value data in this direction. (b) A block of cells containing part of the interface. A fit for the cell in the center (yellow) is made. The neighbouring cell height function values, are used to reconstruct the interface.

A graphical representation of the fitting is shown in figure $6.2 \mathrm{~b}$. For a local cell $\mathscr{C}$, the second order polynomial is of the form:

$$
y(x)=a x^{2}+b x+c,
$$

with coefficients:

$$
\begin{aligned}
& a=\frac{y_{1}\left(x_{2}-x_{3}\right)+y_{2}\left(x_{3}-x_{1}\right)+y_{3}\left(x_{1}-x_{2}\right)}{x_{1}^{2}\left(x_{2}-x_{3}\right)+x_{2}^{2}\left(x_{3}-x_{1}\right)+x_{3}^{2}\left(x_{1}-x_{2}\right)}, \\
& b=\frac{y_{1}\left(x_{2}^{2}-x_{3}^{2}\right)+y_{2}\left(x_{3}^{2}-x_{1}^{2}\right)+y_{3}\left(x_{1}^{2}-x_{2}^{2}\right)}{x_{1}\left(x_{2}^{2}-x_{3}^{2}\right)+x_{2}\left(x_{3}^{2}-x_{1}^{2}\right)+x_{3}\left(x_{1}^{2}-x_{2}^{2}\right)} \\
& c=\frac{y_{1} x_{2} x_{3}\left(x_{2}-x_{3}\right)+y_{2} x_{1} x_{3}\left(x_{3}-x_{1}\right)+y_{3} x_{1} x_{2}\left(x_{1}-x_{2}\right)}{x_{2} x_{3}\left(x_{2}-x_{3}\right)+x_{1} x_{3}\left(x_{3}-x_{1}\right)+x_{1} x_{2}\left(x_{1}-x_{2}\right)} .
\end{aligned}
$$

The position of each interface point $\mathscr{I}$ here is given by $\left(x_{i}, y_{i}+\Delta h\right)$, where $x, y$ are the positions of the cell center and $\Delta$ is the cell size.

Two events can occur:

- The height function data for the $y$-direction is known for all neighbouring cells $\mathscr{N}$ of the cell of interest $\mathscr{C}$. In this case, the origin of the polynomial fit can be 
chosen at the cell center. This reduces the coefficients to:

$$
\begin{aligned}
& a=\frac{y_{1}-2 y_{2}+y_{3}}{2 \Delta^{2}}, \\
& b=\frac{y_{3}-y_{1}}{2 \Delta}, \\
& c=y_{2} .
\end{aligned}
$$

Now, $x_{1}=-\Delta, x_{2}=0$ and $x_{3}=\Delta$ and $y_{1}, y_{2}$ and $y_{3}$ are given by the height functions in the cells: $y_{i}=\Delta \cdot h$.

- The height function data is unknown for one of the neighboring cells $\mathscr{N}$. Now, if this $\mathscr{N}$ is an interface cell, the height function value in $x$-direction is used to construct $\mathscr{I}$. If $\mathscr{N}$ is not an interface cell, the adjacent cells are searched for height function values, up to a $5 \times 5$ stencil centered in $\mathscr{C}$. If this stencil does not contain the necessary height function data, either re-meshing is required, or destruction of the fluid takes place. This can for instance occur for fluid fractions smaller than $2 \Delta$.

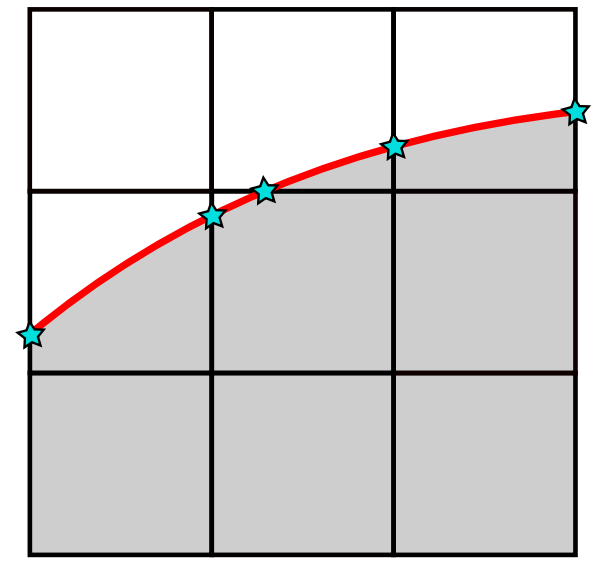

(a)

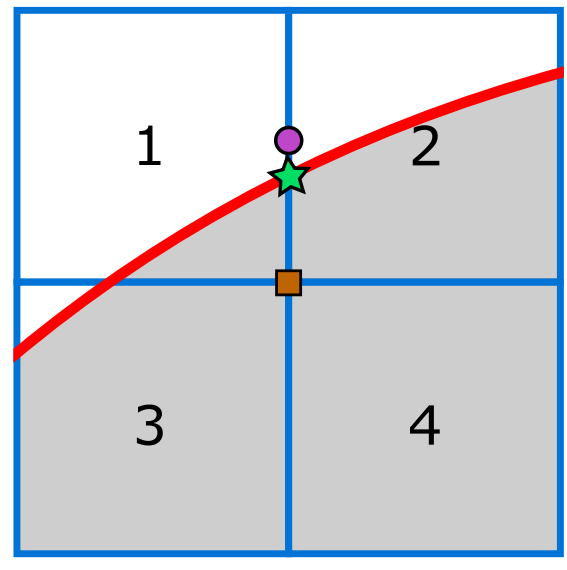

(b)

Figure 6.3: Reconstruction of the surface tension where the interface crosses the face cells. (a) The interface points where the value of the surface tension is evaluated. (b) Method to calculate the surface tension. The surface tension at the interface crossing the cell face purple circle is calculated by the average value of the surface tensions stored in cells 1 and 2, the brown square by the average value of the surface tensions stored in cells 1 to 4 . The surface tension at the interface crossing the cell face $\mathscr{P}$ (green star) is then found by interpolation of the values of the purple circle and brown square. 


\subsection{Validation}

To calculate the surface tension acceleration of equation 6.7, we first determine the tangential vector in $\mathscr{C}$, by the average slope of equation 6.8: $\mathbf{t}=a\left(x_{\text {left }}+x_{\text {right }}\right)+b$, normalised such that $\|\mathbf{t}\|_{2}=1$. The positions $x_{\text {left,right }}$ where the interface crosses the cell faces, are at $x= \pm \Delta / 2$ if the neighboring cells are interface cells. Otherwise $x_{\text {left,right }}$ is found by solving equation 6.8 for $x$ at $y= \pm \Delta / 2$.

The surface tension values $\sigma_{\text {left,right }}$ are obtained on the same positions $x_{\text {left,right }}$, see figure 6.3a. Take for instance the interface crossing $\mathscr{P}=x_{\text {right }}, y_{\text {right }}$. As the surface tension data is stored in the cell centers, an interpolation from the cell centers to $\mathscr{P}$ is required. The four nearest cells around $\mathscr{P}$ are used for this interpolation, see figure $6.3 \mathrm{~b}$. First, the average surface tension in the two adjacent cells is calculated (the purple point, centered between cell 1 and 2), as well as the surface tension in the center of the four cells (the brown square). Now, the surface tension in $\mathscr{P}$ is calculated by:

$$
\sigma_{\mathscr{P}}=\gamma \sigma_{\text {purple }}+(1-\gamma) \sigma_{\text {brown }}
$$

where $\gamma$ depends on the position of $\mathscr{P}$ and is a value between 0 and 1 . The purple and green point overlap when $\gamma=1$.

The obtained tangential vector and surface tension values are used in equation 6.7, adding the required acceleration term for Marangoni stresses to the Navier-Stokes equations (equation 6.2).

\subsection{Validation}
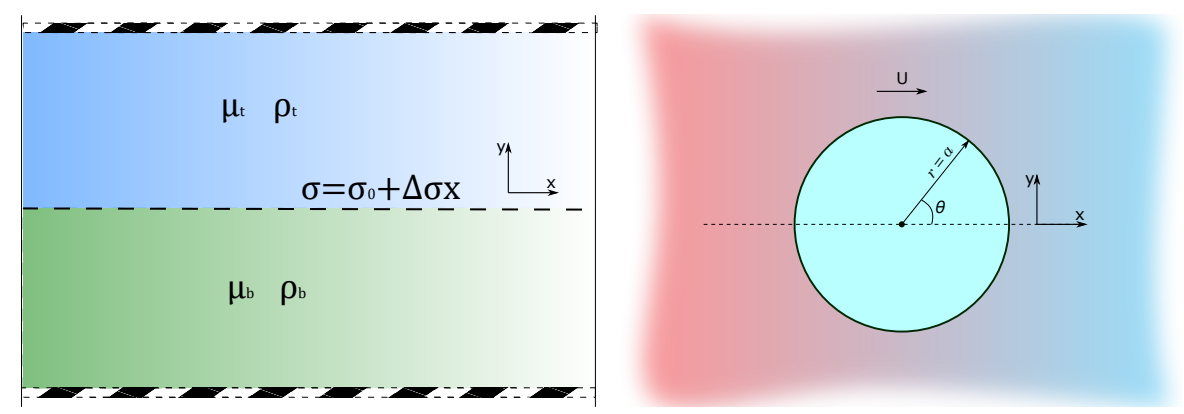

(a)

(b)

Figure 6.4: The two validation cases. (a) Initial configuration of 2 liquids stacked on top of each other in beween 2 walls at $y= \pm b$, under a gradient field of surface tension. (b) Initial condition of a 2-dimensional droplet of radius $a$ in an imposed temperature gradient field, represented by the color gradient in the background. 
The previously described method is validated against two situations shown in figure 6.4, for which we derive analytical results for the steady state case. First two immiscible liquids on top of each other in a thermal gradient are examined. Second a single droplet moving due to a surface tension gradient is compared to an analytical solution.

\subsubsection{Two liquid layers in a thermal field}

Two immiscible fluids are stacked on top of each other in an infinitely long channel with walls at $y= \pm b$, see figure 6.4(a). A gradient of surface tension $\sigma$ is applied on the interface between the liquids, at $y=0$, as:

$$
\sigma(x, y)=\sigma_{0}+\Delta \sigma x,
$$

where $\sigma_{0}$ is an offset surface tension and $\Delta \sigma$ the surface tension gradient. For the steady state case, an analytical solution can be found, see section 6 .A. The resulting steady state velocity profile is:

$$
u_{x}(y)=-\frac{\Delta \sigma}{\mu_{t}+\mu_{b}}|y|+\frac{\Delta \sigma}{\mu_{t}+\mu_{b}} b
$$

A numerical domain of size $1 \mathrm{~cm} \times 1 \mathrm{~cm}$ centered around $\mathrm{y}=0(x \in[-0.5,0.5]$ and $y \in[-0.5,0.5])$ is taken. The viscosity $\mu$ and the density $\rho$ are taken to be equal for both fluids. The values chosen for both fluids in the system are given by table 6.1.

Table 6.1: The values for the liquid stacked on each other.

\begin{tabular}{|c||c|}
\hline parameter & value \\
\hline \hline Length $L$ & $0.01 \mathrm{~m}$ \\
Density $\rho$ & $420 \mathrm{~kg} \mathrm{~m}^{-3}$ \\
Viscosity $\mu$ & $0.08 \mathrm{~Pa} \mathrm{~s}^{-1}$ \\
Surface tension $\sigma_{0}$ & $0.01 \mathrm{Nm}^{-1}$ \\
Surface tension gradient $\Delta \sigma$ & $8 \cdot 10^{-3} \mathrm{Nm}^{-2}$ \\
\hline
\end{tabular}

The simulated velocity is given in figure 6.5 , for a variety of grid levels $l$. The amount of elements $n$ is given by $n=2^{2 l}$. The steady state velocity is shown by the dashed line in the figure. The results of this simulation show that a fluid flow is correctly generated along the interface of the fluids, due to the presence of the tangential surface tension acceleration. The Marangoni stresses leads to mass transfer of the fluid along the interface, which eventually drives the fluid in the whole domain and converges towards the analytically calculated steady state velocity. The highest grid level, with $512 \times 512$ elements is slowly approaching the steady state velocity, 


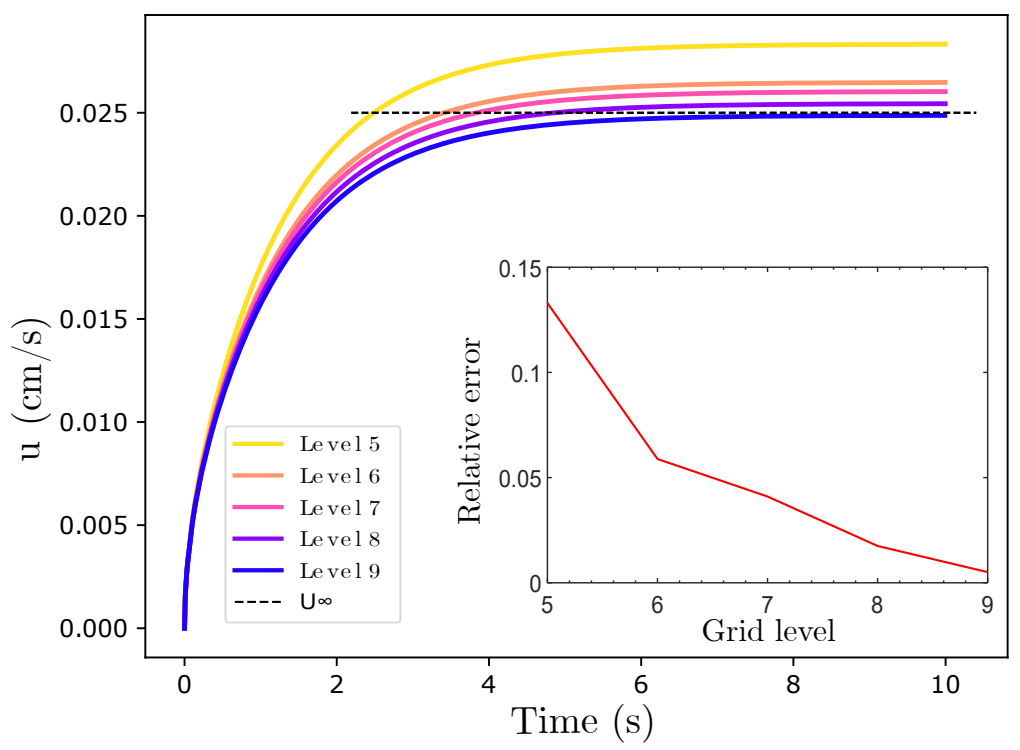

Figure 6.5: The interface velocity plotted against time for different grid levels $l$. The amount of cells used are $n=2^{2 l}$. The dashed line represents the analytical steady state velocity of equation 6.13. The inset shows the relative error $\left(U_{t 10}-U \infty\right) / U \infty$.

Table 6.2: parameters used in the droplet simulations for figure 6.7

\begin{tabular}{l||llllll}
$a$ & 0.15 & 0.12 & 0.1 & 0.085 & 0.075 & 0.06 \\
\hline$\mu$ & 0.001 & 0.004 & 0.01 & 0.02 & 0.05 & \\
\hline$\Delta \sigma$ & 0.01 & 0.005 & 0.002 & 0.001 & 0.0005 &
\end{tabular}

but stopped after the maximum simulation time of 10 seconds. The inset of the figure shows the relative error $\left(U_{t 10}-U \infty\right) / U \infty$, where $U_{t 10}$ is the velocity of the simulation at the interface after 10 seconds and the infinite velocity is given by the analytical velocity $U \infty=u_{x}(y=0)$. Here it is clearly shown that the error reduces significantly with increasing grid level.

\subsubsection{Drop migration}

The first test case shows that the tangential surface tension acceleration implementation works well for the behavior of straight surfaces. A second test case for a curved interface is performed in this section. The curvature of the interface introduces a normal surface tension acceleration on top of the tangential surface tension. Here, we consider a liquid circular cylinder (or 2-dimensional droplet) of radius $a$, inside a 
secondary liquid medium (see figure 6.4(b). The liquids are assumed to be immiscible. A constant temperature gradient field is applied, leading to a gradient in surface tension:

$$
\sigma(x, y)=\sigma_{0}+\Delta \sigma x .
$$

The gradient of surface tension will cause a motion of the fluid around and inside the droplet. The change in surface tension, along surface of the droplet $\Delta \sigma$, is assumed to be relatively small compared to the average surface tension $\sigma_{0}$, such that the droplet can be assumed to remain spherical with a constant radius $a\left(\Delta \sigma a \ll \sigma_{0}\right)$. This problem has an analytical solution. For the 3-dimensional case it was derived in Ref. [36]. The solution for the 2-dimensional problem is shown in section 6.B. Assuming that the inner and outer viscosity $\mu$ are equal, the steady-state migration velocity $U_{\infty}$ of the droplet is:

$$
U_{\infty}=\frac{\Delta \sigma}{8 \mu} a .
$$

For the numerical calculation, a droplet is placed in a square domain with size $1 \times 1$. At the start of the simulation all the fluids in the system are in rest. With time the fluids start moving, due to the presence of a surface tension gradient. The fluid flow in and around the droplet causes a movement in horizontal direction of the droplet. The droplet will be accelerating until it reaches a steady-state velocity. Figure 6.6 shows the velocity profile at steady state of both the analytical (Figure 6.6(a)) and numerical (Figure 6.6(b)) results. The simulated velocity is similar to that of the analytical result. However, at the boundaries of the domain a small difference in the velocity field can be observed, due to the boundary conditions. These have a small influence on the droplet behavior.

We now focus on the steady state migration velocity $U_{\infty}$ of the droplet. The numerical results for a wide range of parameters have been calculated, given in table 6.2. Some of these parameter combinations are expected to result in the same droplet velocities, hereby testing the robustness of our numerical method. A wider domain, of size $2 \times 1$, is chosen to reduce the influence of the boundary conditions on the droplet velocity. The results of the center of mass migration $x$-velocity are presented in Figure 6.7. The numerical result shows a fluctuation around the steady-state velocity, see figure 6.8(a). The wavelength of the oscillations is in the order of the cell size in the numerical domain. The oscillations seem to originate from the normal component of the surface tension, see figure 6.8(b). Whereas the net acceleration due to the tangential component is relatively constant, a more pronounced oscillation is observed for the acceleration due to the normal component of surface tension. Since the normal and tangential component are based on different methods, the inner product of the 


\subsection{Validation}
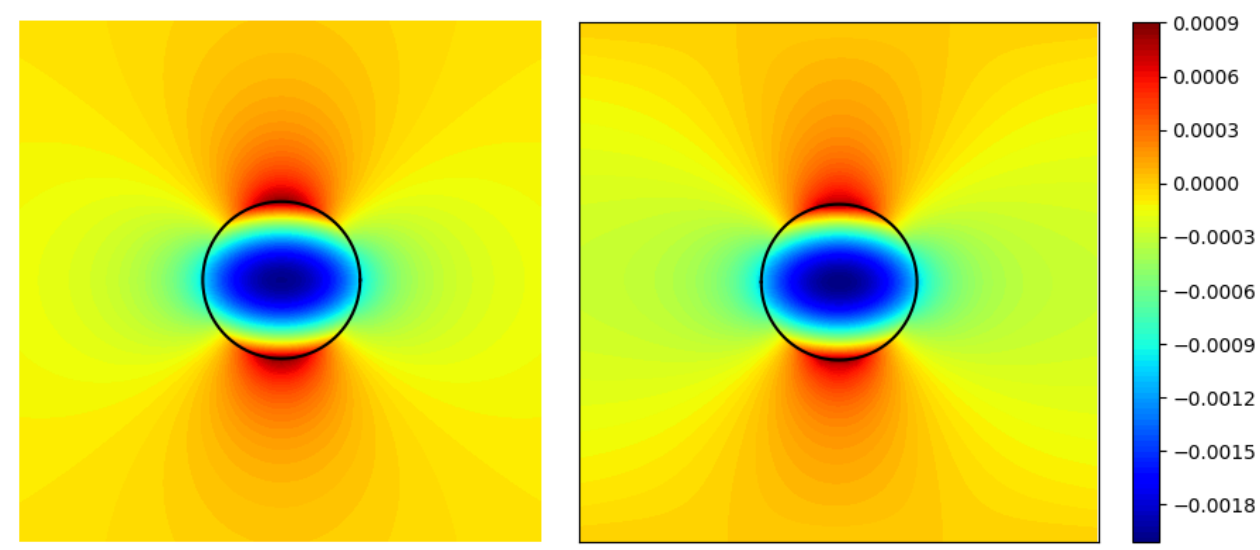

(a)

(b)

Figure 6.6: Velocity in the x-direction of a droplet (black) in a constant gradient field of surface tension. The simulation is made with the dimensionless values: $a=0.15$, $x, y \in[-0.5,0.5], p_{0}=0, \sigma_{0}=0.01, \Delta \sigma=5 \cdot 10^{-4}$ and $\mu^{\prime}=\mu=0.01$. (a) Analytical solution, equation 6.48. (b) Numerical result. The color-code indicates the dimensionless velocity.

vectors can be non-zero. The error in the tangential surface tension acceleration is small compared to that of the normal surface tension acceleration. In spite of these shortcomings, the numerical results compare very well to the expected analytical migration velocity, equation 6.15 , for a wide range of parameters. 


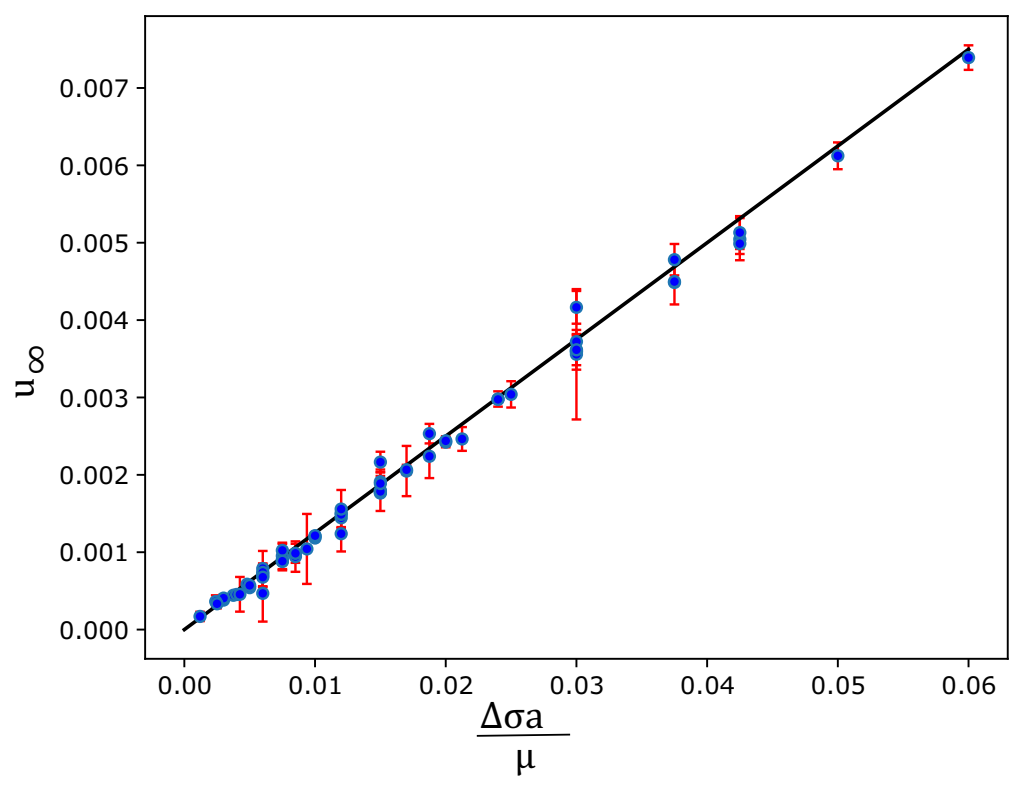

Figure 6.7: The steady-state migration velocity $U_{\infty}$ of the droplets against $\frac{\Delta \sigma a}{\mu}$. The black line indicates the $1 / 8$ ratio from equation 6.15 . The error bars in the figure are a result of the fluctuations in velocity.

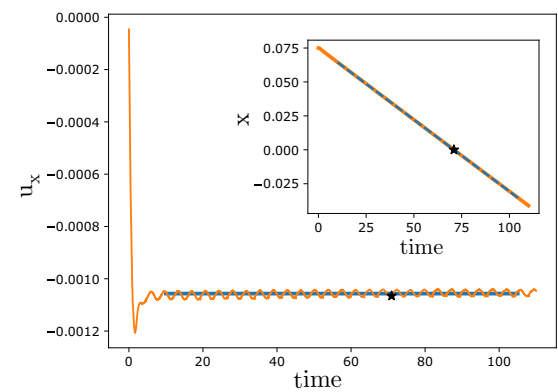

(a)

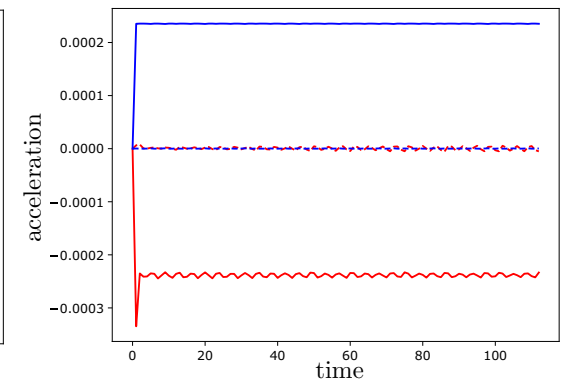

(b)

Figure 6.8: The origin of the velocity oscillations. (a) Droplet center of mass dimensionless $\mathrm{x}$-velocity versus dimensionless time. The inset contains the center of mass dimensionless $\mathrm{x}$-position of the droplet versus dimensionless time. The blue line is a fit to the position data. The black star denotes the position where the droplet reaches the center of the simulation grid. (b) Net dimensionless acceleration in versus dimensionless time, due to normal (red) and tangential (blue) surface tension. The accelerations in the $x$-direction are solid lines, whereas the dashed lines represent accelerations in the $y$-direction. 


\subsection{Conclusion and outlook}

A numerical method with a locally refined tangential surface tension is implemented in Basilisk. A local second order fit of the interface is made based on the VoF data. Height functions are used to obtain 3 interface points to locally fit the interface between fluids in a finite volume cell.

The implementation has been validated by two test cases. First, for two stacked viscous fluids, the numerical method converges towards the analytical result with increasing mesh resolution. Second, for a droplet moving due to a surface tension gradient, the velocity field of the droplet compares well with the analytical solution. The droplet velocity against time showed a periodic fluctuation in the velocity. This oscillation was explained with the net acceleration of the droplet, which shows that the oscillation are due to the normal component of the surface tension.

In the current implementation, the inner product of the tangential vector and the normal vector are non-zero. However, simulations show that this does not have a large effect on the simulation of the tangential surface tension acceleration. The error in the tangential component is small compared to the normal component of surface tension acceleration. In future work, an improved implementation of the normal force due to the surface tension is required to obtain more accurate results. Using a similar local fitting approach, the tangential vector can be made perpendicular to the normal vector. 


\section{A Analytical results to the stacked liquid layers}

A steady state solution, for the problem described in subsection 6.3.1, can be calculated. We start by the assumption that interface between the fluids stays flat, implying there is no pressure build-up and the tangential surface tension acceleration along the surface is constant. Then, the Navier-Stokes equation reduces to:

$$
\mu \nabla^{2} \mathbf{u}=0
$$

The boundary conditions at the walls are:

$$
u(y= \pm b)=0 .
$$

Furthermore, at the interface, the velocity of both fluids should be equal:

$$
u^{+}=u^{-}
$$

The \pm denote the velocity just above $(+)$ or below $(-)$ the interface at $y=0$. Combining equations 6.16 and 6.18 , result in:

$$
u_{x}= \begin{cases}a^{-} y+c & \text { for } y \leq 0 \\ a^{+} y+c & \text { for } y>0\end{cases}
$$

The boundary conditions at the wall, equation 6.17 lead to $a^{+}=-a^{-}$and $c=-a^{+} b$.At the interface the stress balance between the viscous and the surface tension stress is given by:

$$
\mu_{t} \partial_{y} u_{x}^{+}-\mu_{b} \partial_{y} u_{x}^{-}=-\Delta \sigma
$$

Substituting equation 6.19 into equation 6.20 gives:

$$
a_{t}=-\frac{\Delta \sigma}{\mu_{t}+\mu_{b}} .
$$

The velocity can then be expressed as:

$$
u_{x}(y)=-\frac{\Delta \sigma}{\mu_{t}+\mu_{b}}|y|+\frac{\Delta \sigma}{\mu_{t}+\mu_{b}} b
$$

\section{B Analytical results to the migrating droplet in $2 \mathrm{D}$.}

Here an analytical solution for the migrating droplet of radius $a$, presented in section 6.3.2 is given. We follow the derivation of the $3 \mathrm{D}$ migration problem, given in Ref. [37]. 
The fluid properties below $u, \rho, \mu, \ldots$ are notated without prime outside the drop, and their counterparts inside the droplet are denoted with a prime, [ $\left.{ }^{\prime}\right]$. The NavierStokes equations for the steady motion of the liquid in the medium and droplet has the form:

$$
\begin{gathered}
\nabla p=\mu \nabla^{2} \mathbf{u}, \\
\nabla p^{\prime}=\mu^{\prime} \nabla^{2} \mathbf{u}^{\prime},
\end{gathered}
$$

where $p$ is the liquid pressure, $u$ the velocity and $\mu$ the viscosity of the liquid. Taking into account the symmetry of the problem, a polar coordinate system is used with the origin placed in the center of the drop. In polar coordinates the NS equations become:

$$
\begin{array}{rlrl}
\hat{\mathbf{r}}: & \frac{\partial p}{\partial r} & =\mu\left(\frac{1}{r} \frac{\partial}{\partial r}\left(r \frac{\partial u_{r}}{\partial r}\right)+\frac{1}{r^{2}} \frac{\partial^{2} u_{r}}{\partial \theta^{2}}-\frac{u_{r}}{r^{2}}-\frac{2}{r^{2}} \frac{\partial u_{\theta}}{\partial \theta}\right) \\
\hat{\theta}: & \frac{1}{r} \frac{\partial p}{\partial \theta}=\mu\left(\frac{1}{r} \frac{\partial}{\partial r}\left(r \frac{\partial u_{\theta}}{\partial r}\right)+\frac{1}{r^{2}} \frac{\partial^{2} u_{\theta}}{\partial \theta^{2}}-\frac{u_{\theta}}{r^{2}}+\frac{2}{r^{2}} \frac{\partial u_{r}}{\partial \theta}\right)
\end{array}
$$

The continuity equation reads:

$$
\frac{1}{r} \frac{\partial}{\partial r}\left(r u_{r}\right)+\frac{1}{r} \frac{\partial u_{\theta}}{\partial \theta}=0
$$

The equations of motion for inside the droplet have similar forms to the equations 6.25 and 6.26 .

The coordinate system is assumed to move with the center of mass of the droplet, the outer liquid moves at a velocity $-U$, as shown in figure 6.4. In this system the outer fluid far away from the droplet has velocity distribution:

$$
\begin{aligned}
& u_{r}=U \cos \theta \\
& u_{\theta}=-U \sin \theta
\end{aligned} \quad \text { as } r \rightarrow \infty
$$

At the liquid interface the normal component $T_{r r}$ and the tangential component $T_{r \theta}$ of the stress should be continuous. So $T_{r r}=T_{r r}^{\prime}$ and $T_{r \theta}=T_{r \theta}^{\prime}$ at $r=a$ :

$$
\begin{array}{ll}
T_{r r}: & -p+2 \mu\left(\frac{\partial u_{r}}{\partial r}\right)_{r=a}=-p^{\prime}+2 \mu\left(\frac{\partial u_{r}^{\prime}}{\partial r}\right)_{r=a}+\frac{\sigma_{0}}{a}+\Delta \sigma \cos \theta \\
T_{r \theta}: & \mu\left(r \frac{\partial}{\partial r}\left(\frac{u_{\theta}}{r}\right)+\frac{1}{r} \frac{\partial u_{r}}{\partial \theta}\right)_{r=a}=\mu^{\prime}\left(r \frac{\partial}{\partial r}\left(\frac{u_{\theta}^{\prime}}{r}\right)+\frac{1}{r} \frac{\partial u_{r}^{\prime}}{\partial \theta}\right)_{r=a}-\frac{1}{a} \frac{\partial \sigma}{\partial \theta}
\end{array}
$$

Additionally the velocity should satisfy the following conditions:

$$
u_{r}=u_{r}^{\prime}=0 \quad \text { at } r=a,
$$


because the droplet does not deform and there is no flow through the surface of the droplet due to the fluids being immiscible. The tangential velocity component satisfies the no slip condition along the interface, resulting in:

$$
u_{\theta}=u_{\theta}^{\prime} \quad \text { at } r=a .
$$

Finally, the velocity for all points in the droplet has to be finite. Particularly at the center of the drop:

$$
u_{r}^{\prime} \text { and } u_{\theta}^{\prime} \text { finite at } r=0 .
$$

The equations 6.25 and 6.26 need to be solved using the boundary conditions from equation 6.27 till 6.31, to obtain an expression for the velocities and the pressure inside and outside the drop. The boundaries at infinity, equation 6.27 , suggest a solution of the form:

$$
u_{r}=f(r) \cos \theta, \quad u_{\theta}=g(r) \sin \theta, \quad p=\mu h(r) \cos \theta+p_{0}
$$

Similarly inside the drop:

$$
u_{r}^{\prime}=f^{\prime}(r) \cos \theta, \quad u_{\theta}^{\prime}=g^{\prime}(r) \sin \theta, \quad p^{\prime}=\mu^{\prime} h^{\prime}(r) \cos \theta+p_{0}^{\prime}
$$

Substituting these solutions into equations 6.25 and 6.26 :

$$
\begin{aligned}
\frac{\partial h}{\partial r} & =\frac{\partial^{2} f}{\partial r^{2}}+\frac{1}{r} \frac{\partial f}{\partial r}-\frac{2}{r^{2}}(f+g) \\
-\frac{h}{r} & =\frac{\partial^{2} g}{\partial r^{2}}+\frac{1}{r} \frac{\partial g}{\partial r}-\frac{2}{r^{2}}(f+g) \\
0 & =\frac{\partial f}{\partial r}+\frac{f+g}{r}
\end{aligned}
$$

Substituting equation $6.34 \mathrm{c}$ and equation $6.34 \mathrm{~b}$ into equation $6.34 \mathrm{a}$ leads to:

$$
r^{2} \frac{\partial^{4} f}{\partial r^{4}}+6 r \frac{\partial^{3} f}{\partial r^{3}}+3 \frac{\partial^{2} f}{\partial r^{2}}-\frac{3}{r} \frac{\partial f}{\partial r}=0 .
$$

This is an Euler equation which has solutions of the form:

$$
f \approx r^{n}
$$

Substituting this into equation 6.35 gives the values for $n$ as:

$$
n^{2}(n-2)(n+2)=0,
$$

with roots:

$$
n_{1}=-2, \quad n_{2}=2, \quad n_{3}=0 .
$$


6.B. Analytical results to the migrating droplet in $2 D$.

$f$ can be written as the linear combination of the individual solutions:

$$
f(r)=\frac{b_{1}}{r^{2}}+b_{2} r^{2}+b_{3}
$$

Using this expression for $f$ in equations 6.34 a to $6.34 \mathrm{c}$ :

$$
\begin{array}{r}
g(r)=\frac{b_{1}}{r^{2}}-3 b_{2} r^{2}-b_{3} \\
h(r)=8 b_{2} r .
\end{array}
$$

The general solution to equations 6.25 and 6.26 is then:

$$
\begin{gathered}
u_{r}=\left(\frac{b_{1}}{r^{2}}+b_{2} r^{2}+b_{3}\right) \cos \theta, \quad u_{\theta}=\left(\frac{b_{1}}{r^{2}}-3 b_{2} r^{2}-b_{3}\right) \sin \theta, \\
p=\mu\left(8 b_{2} r\right) \cos \theta+p_{0}
\end{gathered}
$$

Outside the droplet, equation 6.27 , needs to be satisfied, which can only be the case if $b_{2}=0$, otherwise $u_{r} \rightarrow \infty$ and $u_{\theta} \rightarrow \infty$ as $r \rightarrow \infty$. Furthermore, $b_{3}=U$, because $\frac{b_{1}}{r^{2}} \rightarrow 0$ as $r \rightarrow \infty$. The relations for the fluid flow outside the droplet becomes:

$$
u_{r}=\left(\frac{b_{1}}{r^{2}}+U\right) \cos \theta, \quad u_{\theta}=\left(\frac{b_{1}}{r^{2}}-U\right) \sin \theta, \quad p=p_{0}
$$

For the inside of the droplet, the boundary condition of finite velocity inside the drop, given by equation 6.31 , implies that $b_{1}^{\prime}=0$. The velocities and pressure are then

$$
\begin{gathered}
u_{r}^{\prime}=\left(b_{2}^{\prime} r^{2}+b_{3}^{\prime}\right) \cos \theta, \quad u_{\theta}^{\prime}=\left(-3 b_{2}^{\prime} r^{2}-b_{3}^{\prime}\right) \sin \theta, \\
p^{\prime}=\mu^{\prime}\left(8 b_{2}^{\prime} r\right) \cos \theta+p_{0}^{\prime},
\end{gathered}
$$

for the liquid inside the drop. The stress in the normal direction, given by equation $6.28 \mathrm{a}$, of the surface results in a relation between $b_{2}^{\prime}$ and $b_{1}$ :

$$
-p_{0}-4 \mu \frac{b_{1}}{a^{3}} \cos \theta=-p_{0}^{\prime}-4 \mu^{\prime} b_{2}^{\prime} a \cos \theta+\frac{\Delta \sigma}{a}+\Delta \sigma \cos \theta,
$$

which should hold for all values of $\theta$, leading to:

$$
b_{1}=\frac{\mu^{\prime}}{\mu} b_{2}^{\prime} a^{4}-\frac{\Delta \sigma}{4 \mu} a^{3} .
$$

The only two boundary conditions that are not yet used are the no slip between the liquids, equation 6.30, and the absence of radial directional flow at the interface, 
equation 6.29:

$$
\begin{aligned}
\left.u_{r}\right|_{r=a}=0 & \Rightarrow \frac{b_{1}}{a^{2}}=-U, \\
\left.u_{r}^{\prime}\right|_{r=a}=0 & \Rightarrow-b_{2}^{\prime} a^{2}-b_{3}^{\prime}=0 \\
u_{\theta}=u_{\theta}^{\prime} & \Rightarrow \frac{b_{1}}{a^{2}}-U=-3 b_{2}^{\prime} a^{2}-b_{3}^{\prime}
\end{aligned}
$$

substituting these conditions into equation 6.45 results in:

$$
U=\frac{\sigma_{1}}{4\left(\mu+\mu^{\prime}\right)} a
$$

and the velocities and pressure for the complete flow field are then given by:

$$
\begin{array}{cl} 
& u_{r}=U\left(1-\frac{a^{2}}{r^{2}}\right) \cos \theta, \quad u_{\theta}=-U\left(1+\frac{a^{2}}{r^{2}}\right) \sin \theta, \\
\text { outside: } & \\
& u_{r}^{\prime}=-U\left(1-\frac{r^{2}}{a^{2}}\right) \cos \theta, \quad u_{\theta}^{\prime}=U\left(1-3 \frac{r^{2}}{a^{2}}\right) \sin \theta, \\
\text { inside: } & p^{\prime}=p_{0}+\frac{\sigma_{0}}{a}-8 \mu^{\prime} \frac{r}{a^{2}} U \cos \theta
\end{array}
$$

A plot of this solution is presented in figure 6.9. The pressure field clearly shows the that the Laplace pressure is much larger than the added pressure due to tangential surface stress. The velocity field in $x$-direction is symmetrical along $y=0$, as expected from the condition of the surface tension gradient. 


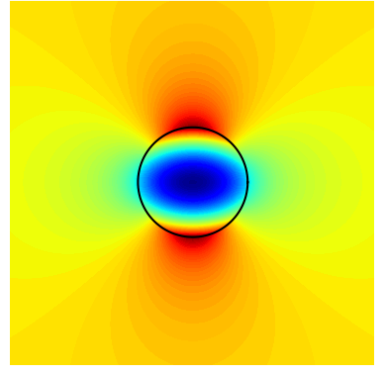

(a)

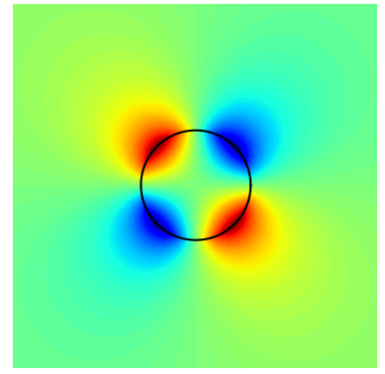

(b)

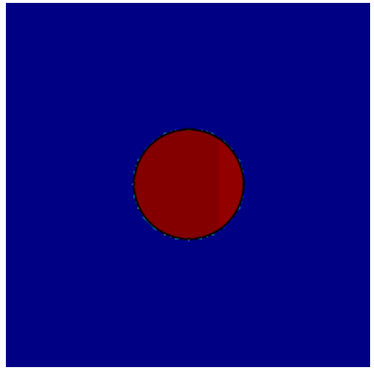

(c)

Figure 6.9: Analytic solution of the drop, with dimensionless values: $a=0.15$, $x, y \in[-0.5,0.5], p_{0}=0, \sigma_{0}=0.01, \Delta \sigma=5 \cdot 10^{-4}$ and $\mu^{\prime}=\mu=0.01$. (a) Velocity in $\mathrm{x}$-direction. (b) Velocity in y-direction. (c) Pressure.

\section{References}

[1] B. Franklin, W. Brownrigg, and M. Farish, "Of the Stilling of Waves by means of Oil. Extracted from Sundry Letters between Benjamin Franklin, LL. D. F. R. S. William Brownrigg, M. D. F. R. S. and the Reverend Mr. Farish", Philos. Trans. R. Soc. London 64, 445-460 (1774).

[2] L. Rayleigh, "Measurements of the Amount of Oil Necessary in Order to Check the Motions of Camphor upon Water", Proc. R. Soc. London 47, 364-367 (1889).

[3] J. A. Fay, "Physical processes in the spread of oil on a water surface", in International oil spill conference, volume 1971, 463-467 (American Petroleum Institute) (1971).

[4] D. P. Hoult, "Oil Spreading on the Sea", Annu. Rev. Fluid Mech. 4, 341-368 (1972).

[5] M. Foda and R. G. Cox, "The spreading of thin liquid films on a water-air interface", J. Fluid Mech. 101, 33-51 (1980).

[6] D. P. Gaver and J. B. Grotberg, "The dynamics of a localized surfactant on a thin film", J. Fluid Mech. 213, 127-148 (1990).

[7] R. V. Craster and O. K. Matar, "Dynamics and stability of thin liquid films", Rev. Mod. Phys. 81, 1131-1198 (2009).

[8] Y. Yeo, O. A. Basaran, and K. Park, "A new process for making reservoir-type microcapsules using ink-jet technology and interfacial phase separation”, J. Control. Release 93, 161-173 (2003). 
[9] Y. Yeo, A. U. Chen, O. A. Basaran, and K. Park, "Solvent exchange method: A novel microencapsulation technique using dual microdispensers", Pharm. Res. 21, 1419-1427 (2004).

[10] B. Duan, L. A. Hockaday, K. H. Kang, and J. T. Butcher, "3D Bioprinting of heterogeneous aortic valve conduits with alginate/gelatin hydrogels", J. Biomed. Mater. Res. - Part A 101 A, 1255-1264 (2013).

[11] N. Blanco-Pascual, R. B. J. Koldeweij, R. S. A. Stevens, M. P. Montero, M. C. Gómez-Guillén, and A. T. Cate, "Peptide Microencapsulation by Core-Shell Printing Technology for Edible Film Application”, Food Bioprocess Technol. 7, 2472-2483 (2014).

[12] A. T. Ten Cate, C. H. Gaspar, H. L. K. Virtanen, R. S. A. Stevens, R. B. J. Koldeweij, J. T. Olkkonen, C. H. A. Rentrop, and M. H. Smolander, "Printed electronic switch on flexible substrates using printed microcapsules", J. Mater. Sci. 49, 5831-5837 (2014).

[13] M. Hayakawa, H. Onoe, K. H. Nagai, and M. Takinoue, "Complex-shaped threedimensional multi-compartmental microparticles generated by diffusional and Marangoni microflows in centrifugally discharged droplets", Sci. Rep. 6, 20793 (2016).

[14] T. Kamperman, V. D. Trikalitis, M. Karperien, C. W. Visser, and J. Leijten, "Ultrahigh-Throughput Production of Monodisperse and Multifunctional Janus Microparticles Using in-Air Microfluidics", ACS Appl. Mater. Interfaces 10, 23433-23438 (2018).

[15] J.-J. Xu, Z. Li, J. Lowengrub, and H. Zhao, "A level-set method for interfacial flows with surfactant”, J. Comput. Phys. 212, 590-616 (2006).

[16] F. Blanchette and T. P. Bigioni, "Dynamics of drop coalescence at fluid interfaces”, J. Fluid Mech. 620, 333-352 (2009).

[17] F. Blanchette, "Simulation of mixing within drops due to surface tension variations", Phys. Rev. Lett. 105, 13-16 (2010).

[18] K. E. Teigen, P. Song, J. Lowengrub, and A. Voigt, "A diffuse-interface method for two-phase flows with soluble surfactants", J. Comput. Phys. 230, 375-393 (2011).

[19] $\mathrm{H} . \mathrm{Wu}$ and $\mathrm{X} . \mathrm{Xu}$, "Analysis of a diffuse-interface model for the binary viscous incompressible fluids with thermo-induced marangoni effects", Communications in Mathematical Sciences 11, 603-633 (2013). 
References

[20] H. Wu, "Well-posedness of a diffuse-interface model for two-phase incompressible flows with thermo-induced marangoni effect", European Journal of Applied Mathematics 28, 380-434 (2017).

[21] A. J. James and J. Lowengrub, "A surfactant-conserving volume-of-fluid method for interfacial flows with insoluble surfactant", J. Comput. Phys.s 201, 685-722 (2004).

[22] M. Lappa, "Assessment of vof strategies for the analysis of marangoni migration, collisional coagulation of droplets and thermal wake effects in metal alloys under microgravity conditions", Comput. Mater. Continua 2, 51-64 (2005).

[23] Y. Alhendal, A. Turan, and W. I. Aly, "Vof simulation of marangoni flow of gas bubbles in 2d-axisymmetric column", Procedia Computer Science 1, 673-680 (2010).

[24] C. Ma and D. Bothe, "Direct numerical simulation of thermocapillary flow based on the volume of fluid method", Int. J. of Multiphas. Flow 37, 1045-1058 (2011).

[25] S. Fleckenstein and D. Bothe, "Simplified modeling of the influence of surfactants on the rise of bubbles in vof-simulations", Chem. Eng. Sci. 102, 514-523 (2013).

[26] S. Popinet, "An accurate adaptive solver for surface-tension-driven interfacial flows”, J. Comput. Phys. 228, 5838-5866 (2009).

[27] M. M. Francois and B. K. Swartz, "Interface curvature via volume fractions, heights, and mean values on nonuniform rectangular grids", J. Comput. Phys. 229, 527-540 (2010).

[28] A. Leshansky, S. Afkhami, M.-C. Jullien, and P. Tabeling, "Obstructed breakup of slender drops in a microfluidic t junction", Phys. Rev. Lett. 108, 264502 (2012).

[29] J. Philippi, P. Lagrée, and A. Antkowiak, "Drop impact on a solid surface: short time self-similarity”, J. Fluid Mech. 795, 96-135 (2015).

[30] S. Wildeman, C. Visser, C. Sun, and D. Lohse, "On the spreading of impacting drops", J. Fluid Mech. 805, 636-655 (2016).

[31] I. Seric, S. Afkhami, and L. Kondic, "Direct numerical simulation of variable surface tension flows using a Volume-of-Fluid method", J. Comput. Phys. 352 (2017). 
[32] J. U. Brackbill, D. B. Kothe, and C. Zemach, "A continuum method for modeling surface tension", J. Comput. Phys. 100, 335-354 (1992).

[33] S. Popinet, "Gerris: A tree-based adaptive solver for the incompressible Euler equations in complex geometries", J. Comput. Phys. 190, 572-600 (2003).

[34] P. K. Kundu, I. M. Cohen, and D. R. Dowling, Fluid Mechanics (Elsevier) (2016).

[35] S. J. Cummins, M. M. Francois, and D. B. Kothe, "Estimating curvature from volume fractions", Computers \& structures 83, 425-434 (2005).

[36] N. O. Young, J. S. Goldstein, and M. J. Block, "The motion of bubbles in a vertical temperature gradient", Journal of Fluid Mechanics 6, 350-356 (1959).

[37] V. G. Levich, "Physicochemical hydrodynamics", (1963). 




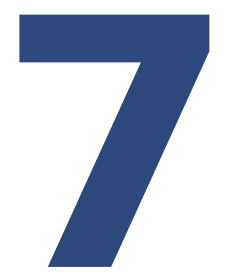

\section{Conclusions and Outlook}

In this thesis we studied the interaction of heterogeneous liquid-liquid systems. To this end, we developed novel experimental and numerical methods.

First, we set out to study the microscopic scale solidification behavior in droplets. In Chapter 2 a novel experimental method was presented, based on total internal reflection. With this nethod we directly visualized nucleation and crystal growth in a spreading droplet. The critical condition for arrest of the contact line was determined by direct observation to depend on the local interactions of crystallites with the contactline. Furthermore the number of crystals growing in a spreading drop follow a power law $n_{\text {crystals }} \propto\left(t / \tau_{c}\right)^{5 / 2}$. Finally we were able to predict the complete early time-dependent solidified area of a droplet whilst spreading.

Then, in Chapter 3 the same experimental method was used to study the impact of droplets. A peculiar freezing morphology was revealed, that originates from the complex interplay between the droplet-scale hydrodynamics and phase-transition effects at sufficiently high substrate undercooling. By combining the theories of large scale hydrodynamics and the microscopic growth of crystals, we showed that this behavior depends on the interplay of the crystals with the hydrodynamic boundary layer. Beyond that, we studied the long time-scale delamination of the solidified splat, which we believed to originate from the transition of a weakly ordered crystalline phase under thermo-mechanical stress.

Subsequently, the larger scale influence of solidification on global droplet impact dynamics was studied using a numerical method in Chapter 4. Here, the height function based VoF method was extended by a mushy zone method to model solidification. This model results in an extension of the isothermal regime diagram for droplet impact 
on supercooled surfaces, showing a wide array of behaviors. Depending on both the Ste and We numbers of the impacting droplets, transitions were found from sticking to bouncing, back to sticking. Next to that, the spreading extent of droplets was investigated. It was shown, that for $\mathrm{We}<10$, only in the retraction phase solidification starts having an effect. A new model was proposed for We $>10$, based on energy conservation arguments, to determine the maximum spreading diameter of solidifying droplets. This is of major interest to industrial applications, to control the size of droplet footprints.

A second heterogeneous effect was studied in the sub-seeding Chapters: the behavior of a liquid-liquid system under varying surface tension. First, in Chapter 5 laser-induced fluorescence was used to study the spreading of binary droplets for a large range of viscosities and surface tensions. We found that for binary droplets, the spreading distance was consistent with $L(t) \approx 0.6 \Delta \sigma^{1 / 2}(\rho \eta)^{-1 / 4} t^{3 / 4}$ for sufficiently low Ohnesorge numbers $(\mathrm{Oh} \lesssim 0.2)$, and moderate viscosity ratios $\left(0.1 \lesssim \eta_{1} / \eta_{2} \lesssim 10\right)$. Interestingly, non-dimensionalizing our results and literature experiments for different surfactants, and miscibilities revealed that a universal power law $\tilde{L}=0.6 \tilde{t}^{3 / 4}$ reasonably captures the spreading behaviour over 10 orders of magnitude in dimensionless time for a variety of geometries.

Finally, in Chapter 6 a new numerical method is proposed to model Marangoni effects. It has been developed such, that it is suitable to both concentration and temperature gradients for bi-fluid systems. The model was validated for two test cases, a flat and a curved interface. The model showed apt comparison to analytical results of Marangoni flow for both test cases.

In this thesis, solidification and Marangoni effects have been studied separately. However in many cases, an interaction between both effects can occur. For systems with large temperature gradients, thermal Marangoni effects will influence the flow behavior before or during solidification. Furthermore systems with a strong temperature dependence of the surface tension need to be studied by combining these effects. Furthermore, in this thesis both microscopic and macroscopic phase change has been shown to strongly influence the large scale behavior of droplets. The transition from one scale to the other can be further investigated to increase understanding of the solidified footprint of single droplets. In industry, and especially in 3D-printing, the interaction of multiple droplets is of interest to create functional products. Here we focused on the behavior of single and binary droplet interactions. The effects of multiple droplets landing on substrates can be very intricate as well. The thermal footprint of already deposited droplets will significantly influence the later stages of deposition. 


\section{Summary}

Additive manufacturing has developed from the production of prototypes towards the direct printing of functional parts. Recent developments include the use of multiple materials during a single additive manufacturing step, especially using inkjet printing. To increase the capabilities even further, understanding of interactions of these materials is required. The main goal of this thesis is to study the influence of heterogeneous interactions in droplets. Of fundamental importance to additive manufacturing, is the solidification and final footprint of printed droplets. In Chapter 2 the interplay between the phase transition effects and the contact-line motion of spreading droplets is investigated. The early solidification patterns and dynamics of spreading hexadecane droplets are revealed employing total internal reflection (TIR) imaging. With this method the conditions leading to the contact-line arrest is determined. The overall nucleation behavior was quantified depending on the applied undercooling of the substrate. By combining the Johnson-Mehl-Avrami-Kolmogorov nucleation theory and scaling relations for the spreading of droplets, the temporal evolution of the solid area fraction was calculated. In Chapter 3 the freezing kinetics during the solidification of a droplet while it impacts on an undercooled surface is investigated by TIR-imaging. At sufficiently high undercooling, a peculiar freezing morphology exists that involves sequential advection of frozen fronts from the center of the droplet to its boundaries. This phenomenon is examined by combining elements of classical nucleation theory to the large-scale hydrodynamics on the droplet scale. It was shown that the interaction of crystal growth with the hydrodynamical boundary layer leads to the periodical advection of solidified crystals. Furthermore, a self-peeling phenomenon of the frozen splat is revealed. This peeling is driven by the thermo-mechanical stresses in the solid- 
ified footprint and the existence of a transient crystalline state during solidification. Of further importance is the modelling of the solidification of droplets. Chapter 4 described the extension of the geometrical Volume-of-Fluid interface method, with an enthalpy-porosity formulation to study solidification. The numerical method is validated against experiments available in the literature and favorably compared to earlier numerical methods. A study of $1 \mathrm{~mm}$ sized tin droplets is performed, showing various impact regimes: deposition, capillary ejection, and (partial) break-up. The dependence of the spreading behavior on the Weber (We) and the Stefan (Ste) number is quantified. At early times, the spreading is found to be similar to that of isothermal droplets, but later the solidification arrests the moving interface. An analytical model is developed to determine the maximum spreading diameter as a function of dimensionless parameters. This model is based on a combination of the hydrodynamics and the solidification rate, and gives good estimates for the maximum droplet diameter of solidifying droplets. Next to droplet solidification, the effect of variable surface tension on the behavior of droplet interactions was investigated. Chapter 5 describes an experimental method using stroboscopic illumination by nanosecond laser pulses. With this, the Marangoni-driven spreading of droplets over droplets is studied. The distance $L(t)$ over which a low-surface-tension drop spreads over a high-surface-tension drop was measured for a large array of liquid properties. This reveals that a power-law $L(t) \sim t^{\alpha}$ with a spreading exponent $\alpha \approx 0.75$, reasonably captures the experiments in this chapter, as well as previous experiments for different geometries, miscibilities, and surface tension modifier for ten orders of magnitude in dimensionless time. Finally, in Chapter 6 a novel methodology for the inclusion of variable surface tension acceleration in a geometrical Volume-of-Fluid method is developed. This method provides an accurate method for computing surface tension gradients based on the local reconstruction of the interface. It is applicable to both temperature and concentration gradients. This model has been validated for two situations, one with two stacked fluids with a flat interface and one with a droplet with a curved interface, showing that the method accurately calculates liquid flow driven by variations in surface tension. 


\section{Samenvatting}

3D-printen heeft zich ontwikkeld van de productie van prototypes tot het volledig direct printen van functionele onderdelen. Recente ontwikkelingen omvatten het gebruik van meerdere materialen gedurende een enkele print-stap, met name door middel van inkjet printen. Om de mogelijkheden verder uit te breiden, is het van belang de interactie van de materialen te begrijpen. Het hoofddoel van dit proefschrift is het bestuderen van de invloed van heterogene interacties in druppels. Van fundamenteel belang voor het 3D-printen is de stolling en de uiteindelijke voetafdruk van geprinte druppels. In hoofdstuk 2 wordt de interactie tussen de fase verandering en de beweging van de contactlijn van een spreidende druppel bestudeerd. The patronen die ontstaan tijdens de eerste fase van stolling en de dynamica van spreidende hexadecaan druppels worden onthuld door middel van visualisatie met totale interne reflectie (TIR). Met deze methode wordt het effect dat leidt tot het stopzetten van de contactlijn bepaald. Verder wordt de algehele nucleatie in de druppel gekwantificeerd, afhankelijk van de onderkoeling van het substraat. Het gestolde oppervlak van een spreidende druppel wordt vervolgens berekend door de Johnson-Mehl-Avrami-Kolmogorov theorie te combineren met schalingsrelaties voor spreidende druppels. In hoofdstuk 3 wordt de bevriezingskinetiek gedurende de stolling van een druppel die botst op een onderkoeld oppervlak bestudeerd met behulp van TIR-visualisatie. Wanneer de onderkoeling sterk genoeg is, treedt er een eigenaardig bevriezingsfront op, gekenmerkt door de sequentiële advectie van bevroren fronten van het centrum van de druppel naar zijn buitenkant. Dit fenomeen wordt onderzocht door elementen van de klassieke nucleatie theorie te combineren met hydrodynamica op de druppelschaal. De interactie van de kristalgroei met de hydrodynamische grenslaag zorgt voor de periodieke advectie van 
gestolde kristallen. Daarnaast wordt een zelf-pel-mechanisme van de druppelvoetprint tentoongesteld. Dit pellen wordt gedreven door de thermo-mechanische spanningen in het gestolde materiaal en de aanwezigheid van een transinte kristallijne staat gedurende de stolling. Naast de experimentele resultaten, is het van belang stolling te kunnen modelleren. Hoofdstuk 4 beschrijft de uitbreiding van een geometrisch Volume-ofFluid model met een enthalpie-porositeitsformulering om stolling te bestuderen. De numerieke methode wordt gevalideerd aan de hand van experimenten beschikbaar in de literatuur en bewijst zich positief ten opzichte van bestande numerieke modellen. Hierna is een studie van $1 \mathrm{~mm}$ grote tin-druppels uitgevoerd. Dit laat een scala aan regimes zien na het botsen van de druppels: de depositie, de capillaire ejectie en de (gedeeltelijke) splitsing. De afhankelijkheid van het spreiden op het Weber (We) en Stefan (Ste) getal is gekwantificeerd. Tijdens de vroege spreiding, is het spreidingsgedrag van stollende druppels gelijk aan dat van druppels in een isotherme omgeving. Later wordt de bewegende contactlijn stopgezet door de stolling. Een analytisch model is ontwikkeld, om de maximale spreidingsdiameter te bepalen als functie van dimensieloze parameters. Dit model is gebaseerd op een combinatie van de hydrodynamica en de mate van stolling. Het geeft een goede indruk van de maximale druppeldiameter van stollende druppels. Buiten het stollen van druppels, is het effect bestudeerd van een verschil in oppervlaktespanning voor de interactie van druppels. Hoofdstuk 5 beschrijft een experimentele methode gebaseerd op stroboscopische verlichting gebruik makend van nanoseconde laser pulsen. Hiermee is de Marangoni-spreiding van druppels over druppels bestudeerd. De afstand $L(t)$ waarmee een druppel met lage oppervlaktespanning spreidt over een druppel met hoge oppervlaktespanning is gemeten voor een grote verscheidenheid aan vloeistofeigenschappen. Dit laat zien dat de spreiding zich behoorlijk goed laat beschrijven aan de hand van een machtsfunctie $L(t) \sim t^{\alpha}$, waarbij $\alpha \approx 0.75$. Dit geldt niet alleen voor de experimenten beschreven in dit hoofdstuk, maar ook voor een verscheidenheid aan experimenten in de literatuur, voor verschillende geometrieën, mengbaarheden en oppervlaktespanningsverschillen. Uiteindelijk wordt in hoofdstuk 6 een nieuwe numerieke methode ontwikkeld om variabele oppervlaktespanning toe te voegen aan een geometrische Volume-of-Fluid methode. De methode biedt een nauwkeurige methode om een gradiënt in oppervlaktespanning uit te rekenen, gebaseerd op het lokaal reconstrueren van het grensvlak tussen twee vloeistoffen. Het is van toepassing op variabele oppervlaktespanning, die is ontstaan door zowel temperatuurgradiënten als door concentratiegradiënten. Het model is gevalideerd door middel van twee situaties, één waarbij het grensvlak tussen twee vloeistoffen recht is en één waarbij het grensvlak gekromd is. De vergelijking met analytische oplossingen voor deze situaties laat zien dat het model nauwkeurig de vloeistofstroming ontstaan door variaties in oppervlaktespanning uitrekent. 


\section{Acknowledgements}

Starting a PhD while working in applied research is different from the more common route. Traveling around the country, splitting my week between Eindhoven and Enschede, and balancing the work at TNO with the research presented in this thesis. The topics presented here have much in common with earlier research in my career. Massimiliano diMuzio first introduced me to droplet solidification in the context of aircraft icing, at that time to understand the statistics behind clouds. With Gerben Pieterse I worked on droplet impacts on a thin liquid surface, resembling the encapsulation of droplets flowing over droplets.

The result of this thesis could not have been come to fruition without the help and support of many people around me. First I would like to thank my promotor Detlef Lohse, for giving me the opportunity to be part of his excellent research group. Even though I worked only part-time at Twente, I learned a great deal through our numerous discussions. You set up a vibrant research group where discussions are encouraged, leading to a large number of fruitful collaborations both inside and outside of Physics of Fluids. Furthermore thank you to the research staff in this group, for being available for discussions at all times. In particular I would like to thank Jacco Snoeijer and Devaraj van der Meer for the useful and fun discussions on all topics ranging from surface tension, contact line dynamics and numerics, to supervising undergraduate and graduate students. Furthermore, a big thank you to all support staff, without whom the group would not function at all. Dennis for all the support and overview in the labs, Martin and Gert-Wim for designing and building the experimental setups. Bas, for your support in both IT and electronics and of course Joanita, for being the beating heart of PoF, making sure we never have to worry about administrative matters. 
This research was part of the ERP programme Nano-manufacturing of TNO. Here I would like to thank several people for giving me the opportunity to perform this research. In particular René for initiating the research, Erwin for the initial support and Hamed for providing the framework within TNO that allowed me to spent the major part of four years on my $\mathrm{PhD}$ research. Pieter for supporting me from the start to the end, during several changes to the EfAM department, and even after our small group switched to nano-instrumentation. Finally Frank for taking over our Thermal Fluid Engineering group during the finalising stage of my thesis.

I was lucky to perform my research with many talented people. First I would like to thank Marise and Rielle for the early work on solidification. Hanneke for your insightful and careful scientific approach. Michiel for your inexhaustible knowledge about TIR. Kirsten to always be open for discussions and your wide interest in any topic going on in the lab. Pallav, you are the most creative and careful experimentalist I have had the pleasure to work with. Claas-Willem, thank you for the reintroduction to encapsulation as well as for numerous discussions on science, mentorship and career planning. For the work on Marangoni effects I had the pleasure to work with two graduate students; Bram and Job, thank you for your tenacity in performing experimental and numerical work on a very complex system.

Colleagues at Physics of Fluids and TNO, thank you for always being around for coffee, lunch breaks and everything else that makes working more pleasurable. Special thanks to my early office-mates at PoF: Ruben, Biljana, Shantanu, Lijun, and Pim. Also Arjan for the many trips sharing experiences both inside and outside physics. At TNO, special thanks to the whole TFE team, Cor, Maurice, Oleksiy, Jurjen, Bas, Dagmar, Ruud, Marlies, Artunç, Nupur, Tahmid and Ruben.

Zonder de ondersteuning van vrienden en familie was deze periode niet zo prettig verlopen. Met name dank voor de schakers van Drienerlo in de Nahand en de tennissers, met wie ik menig training en wedstrijd heb gedeeld.

Bedankt papa en mama, omdat jullie er altijd voor ons zijn. En natuurlijk voor de gezellige avonden in Lichtenvoorde en het voorzien van bed en broodje. Christel en Stèphanie, mijn zusjes, bedankt voor het begrip en ondersteuning.

Iris, natuurlijk de laatste en belangrijkste. Jij hebt me weten af te leiden en inspireren. Dank dat je me al die tijd hebt weten te steunen. 